NBSIR 75.652

EPA-550/8-76.001

\title{
Procedures for Estimating Sound Power From Measurements of Sound Pressure
}

An Experimental Investigation with Application to Noise from Portable Air Compressors

Curtis I. Holmer

National Bureau of Standards

July 1975
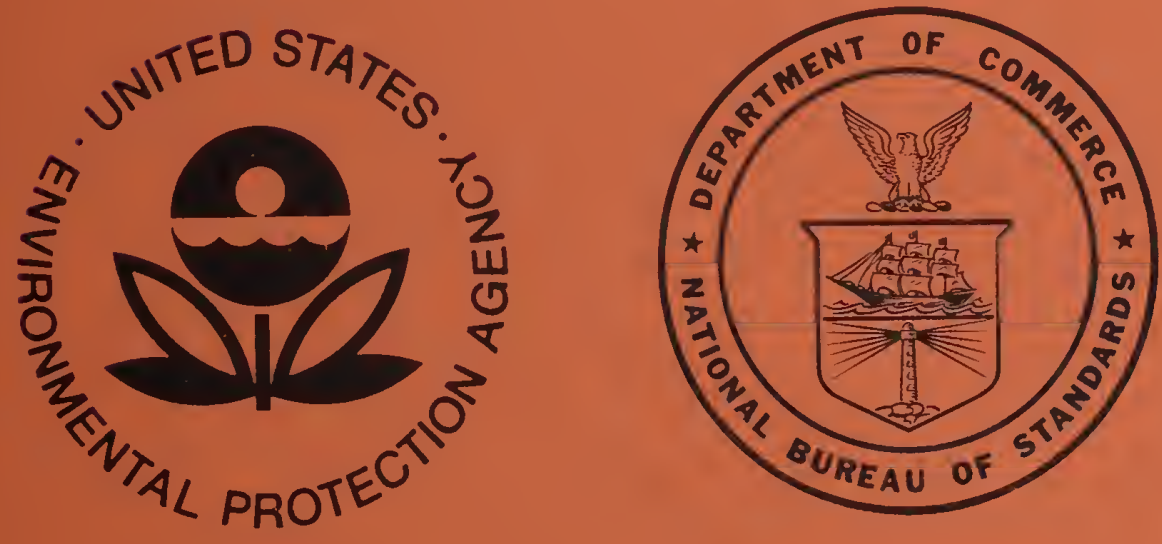

Approved for public release; distribution unlimited

Applied Acoustics Section Institute for Basic Standards National Bureau of Standards Washington. D. C. 20234 
NBSIR 75-652

EPA-550/8-76-001

\section{PROCEDURES FOR ESTIMATING SOUND}

POWER FROM MEASUREMENTS OF SOUND

PRESSURE

An Experimental Investigation with Application to Noise from Portable Air Compressors

Curtis 1. Holmer

Applied Acoustics Section

National Bureau of Standards

Washington, D. C. 20234

July 1975

Final Report

\section{Prepared for}

Office of Noise Abatement and Control

U. S. Environmental Protection Agency

Washington, D. C. 20460

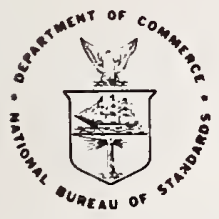

U.S. DEPARTMENT OF COMMERCE, Rogers C.B. Morton, Secrotary James A. Baker, III, Under Secretary Dr. Betsy Ancker-Johnson, Assistant Secretary for Science arid Technology 

1. Introduction 1

2. Experimental Program 3

2.1. Program Objectives and Implementation 3

2.2. Experimental Plan , 3

2.3. Measurement Procedures 3

2.3.1. Measurement Site 3

2.3.2. Instrumentation 4

2.3.3. Test Plan 7

2.3.4. Data Acquisition 9

2.3.5. Data Reduction and Corrections 11

2.4. Description of Compressor Sample 14

2.5. Compressor Operation 15

2.6. Measurement Locations and A-weighted Sound Level Data 16

2.6.1. Far Field Measurements 16

$\begin{array}{ll}\text { 2.6.2. Near Field Measurements } & 17\end{array}$

3. Sound Power Level Calculations 37

3.1. Far Field Sound Power Calculation Procedures 37

3.1.1. Systematic Errors in the Far Field Array 37

3.1.2. Far Field Sound Power Calculations 40

3.2. Near Field Sound Power Calculation Procedures 41

3.3. Sound Power Level Data 44

4. Discussion of Experimental Results 62

4.1. Sound Pressure Level Data 62

4.1.1. Directivity of Compressor Noise 62

4.1.2. Systematic Variation of Noise with Position 62

4.2. Sound Power Level Data 62

5. Analysis of Measurement Error 64

5.1. Introduction 64

5.2. Error in Sound Power Measurement Methodologies 64

5.3. Instrumentation Ac curacy 68

5.4. Total Measurement Error 69

6. Conclusions 71

7. Acknowledgements 72

$\begin{array}{ll}\text { 8. References } & 73\end{array}$ 
Table 1

Table 2

Table 3.1

Table 3.2

Table 3.3

Table 3.4

Table 3.5

Table 3.6

Table 3.7

Table 3.8

Table 3.9

Table 3.10

Table 3.11

Table 3.12

Table 3.13

Table 3.14

Table 3.15

Table 3.16

Table 3.17

Table 3.18

Table 3.19, 20,21

Table 4.1

Table 4.2

Table 5.1

Table 5.2

Table 5.3

Table 5.4

Table 5.5

Table 5.6

Table 5.7

Table 5.8

Table 5.9

Table 5.10

Table 5.11

Table 5.12

Table 5.13

Table 5.14

Table 5.15

Table 5.16

Table 5.17

Table 6

Table 8

Instrumentation data

Description of sources

A-weighted sound pressure level data. Test Number 1

A-weighted sound pressure level data. Test Number 2

A-weighted sound pressure level data. Test Number 3

A-weighted sound pressure level data. Test Number 4

A-weighted sound pressure level data. Test Number 5

A-weighted sound pressure level data. Test Number 6

A-weighted sound pressure level data. Test rumber 7

A-weighted sound pressure level data. Test Number 8

A-weighted sound pressure level data. Test Number 9

A-weighted sound pressure level data. Test Number 10

A-weighted sound pressure level data.

A-weighted sound pressure level data.

A-weighted sound pressure level data.

A-weighted sound pressure level data.

A-weighted sound pressure level data.

A-weighted sound pressure level data.

A-weighted sound pressure level data.

A-weighted sound pressure level data.

(Broad band reference sound source)

Test Number 11

Test Number 12

Test Number 13

Test Number 14

Test Number 15

Test Number 16

Test Number 17

Test Number 18

19

20

21

22

23

24

25 20, 21 (Pure-tone loudspeaker source, $500 \mathrm{~Hz}, 1000 \mathrm{~Hz}$, $2000 \mathrm{~Hz}$ )

Systematic error in estimation of sound power from a compact source using SPL values from a 73 point array on a 7 metre hemisphere (source: pure tone)

Systematic error in estimation of sound power from a

compact source using SPL values from a 73 point array

on a 7 metre hemisphere (source: pink noise).

Sound power level data. Test number 1

Sound power level data. Test number 2

Sound power level data. Test number 3

Sound power level data. Test number 4

Sound power level data. Test number 5

Sound power level data. Test number 6

Sound power level data. Test number 7

Sound power level data. Test number 8

Sound power level data. Test number 9

Sound power level data. Test number 10

Sound power level data. Test number 11

Sound power level data. Test number 12

Sound power level data. Test number 13

Sound power level data. Test number 14

Sound power level data. Test number 15

Sound power level data. Test number 16

Sound power level data. Test number 17

Average deviation and standard deviation of average

deviation of near field sound power level from far

field sound power level for seventeen portable air

compressors. Six near field procedures are shown plus

similar statistics for far field methodology

Table 7 Instruments imprecision (two standard deviations)

associated with commercially available precision

(Type I) sound level meters

Estimated achievable measurement error (90\% confidence

for measurement of A-weighted sound power level of portable

air compressors in a field test environment using a

measurement surface 1 metre from the source surface,

excluding operator error. 
Figure 1 Field test site

Figure 2 Photographs of test site

Figure 3 Equipment configuration for pulse echo tests of test site

Figure 4 Schematic block diagram of data acquisition and analysis instrumentation

Figure 5 Far-field measurement array

Figure 6 Representative near-field measurement positions

Figure 7 Microphone correction factor (Type 4145 cartridge)

Figure 8 Conformal surface at a distance $r$ from a rectangular box

Figure 9 Plot of average deviation of near field from far field sound power level

Figure 10 "Bias" and "precision of A-weighted sound power level vs

Figure Il A-weighted sound power level deviation vs source size 


\section{PROCEDURES FOR ESTIMATING SOUND POWER FROM}

MEASUREMENTS OF SOUND PRESSURE

An Experimental Investigation with Application to Noise From Portable Air Compressors

Curtis I. Holmer

Applied Acoustics Section

Mechanics Division

National Bureau of Standards

Washington, D. C. 20234

\section{ABSTRACT}

This report describes investigations of the accuracy and precision of various measurement methodologies for determining the estimated sound power output of "large" machines in the free field over a reflecting plane. One purpose of this investigation is to place empirical error bounds on many of the free field measurement procedures currently proposed or in use; and in particular, compare the results of "near-field" and "far-field" measurements. The sources used for the investigation included 17 portable air compressors of various types (powered by internal combustion engines), a "reference" sound source, and a loudspeaker driven by a pure tone source. The data recorded include sound pressure level (A-weighted, linear, and 1/3-octave band) on an 84 point hemispherical array of seven metre radius, and "near-field" measurements, sampled every square metre, on a rectangular surface one metre from the machine surface. These data were reduced to provide information on the deviation of "near field" sound power determinations from "far-field" power level (using subsets of the data as appropriate to various methodologies). The measured data for seventeen sources suggests that the value of a sound power estimate based on "near-field" sound pressure level measurements may be an upper bound to the sound power level estimated from far field measurements, subject to the limitations of sampling error. Estimates of total achievable measurement error of A-weighted sound power level of near field determinations relative to far field determinations are made for several measurement methodologies, based on the experimental data.

\section{INTRODUCTION}

This report presents the results of an experimental investigation undertaken by the National Bureau of Standards, of measurement procedures for the determination of sound power output of portable air compressors.

These results are preliminary in the sense that the potential information available from the data bank established in the measurement program has only been partially evaluated. Much additional information remains to be retrieved from the data. The conclusions reached concerning the accuracy and precision of "near-field" sound power measurements are substantiated as far as they go. However, additional analysis of the data may lead to reinterpretation of these findings.

This study was jointly funded by the U. S. Environmental Protection Agency Office of Noise Abatement and Control (EPA/ONAC) and the National Bureau of Standards (NBS) to provide background information for a measurement methodology appropriate for the regulation of noise emission from newly manufactured portable air compressors. EPA supported the cost of data collection and NBS the cost of data reduction and analysis.

True sound power output is conventionally defined as the integral of the normal component of time average acoustic intensity over a surface completely enclosing the source. (See discussion in Section 5.2.) Since the actual measurement process must involve point sampling of the sound field due to the source, and to be widely useful must employ commercially available instrumentation, such measurements can only yield an estimate of true sound power. Present commercially available instrumentation measures mean square sound pressure rather than intensity, so standardized measurement procedures for the estimation of sound power employ measurements of sound pressure under particular controlled situations where the measurements provide data which is known to at least asymptotically approach (in the large radius limit) the scalar magnitude of true intensity.

Present ISO and ANSI standard methods[1] l/ of determination of sound power in the free field over a reflecting plane involve measurements of sound pressure level at points on a hemisphere whose radius is large compared with the largest source dimension ("far field"). Recent draft standards[2] and some

$1 /$ Numbers in brackets refer to references at the end of this report. 
current research[3] suggest that measurements made near the surface of a large machine ("near-field") can also be utilized as the basis for estimates of radiated sound power. This study was intended to: (1) provide empirical evaluation of the suitability of close-in measurements of sound pressure level to infer the "farfield" estimate of sound power output of a portable air compressor; (2) provide empirical estimates of measurement precision and accuracy as a function of the methodology used; and

(3) contribute additional data on noise emission from portable air compressors.

The measurement program consisted of determinations of time averaged sound pressure level at a large number of positions on two different measurement surfaces (one in the "far field" and one in the "near field") surrounding each of seventeen sources. From the data for each source an estimate of the sound power radiated by that source was made for each surface, and the difference between the two measurements was used to infer the validity and accuracy of "near field" measurement procedures relative to the far field procedures. The precision of the measurement procedure was inferred from the statistics of these differences for the set of sources investigated. Since several proposed methodologies include measurement positions which are subsets of the complete set of measurement positions, the accuracy and precision of these methodologies could be inferred in a similar manner.

We recognize that neither of the above measurement procedures provide the absolute sound power level for the reasons already mentioned. As such we recognize that we cannot state with any certainty, the degree to which either sound power determination approximates the absolute sound power output. In the following sections it is emphasized that the phrase "sound power" should be interpreted as refering to the estimate of sound power obtained from measurement of sound pressure levels at large distances from a source. Further, the use of the term "accuracy" is used to describe the relative bias of a determination from the estimate of sound power described above.

The following sections of this report present detailed discussions of: the experimental program, incluaing results of the sound pressure level measurements; the computation of sound power level for a limited number of measurement methodologies, and the results of these computations; estimates of measurement accuracy and precision for the methodologies evaluated; and conclusions regarding the various methodologies. 


\subsection{Program Objectives and Implementation}

The principal objectives of the program were:

1. Test experimentally, the validity of using "near-field" measurements of sound pressure level to predict the "far-field" sound pressure level for large machines. (The computation procedure used involves the intermediate concept of sound power output as a characterization of the source emission.)

2. Provide a data base from which the accuracy and precision of sound power determinations may be estimated based on limited sampling of the sound field.

3. Generate baseline data of noise emission from portable air compressors.

The implementation of these objectives is briefly discussed below.

\subsection{Experimental Plan}

The experiment consisted of the measurement of sound pressure level on two surfaces surrounding the sound source. The larger surface (yielding the "far field" measurement data) was a hemisphere of a fixed 7 metre radius. The sound pressure level was sampled at seven locations utilizing a semicircular microphone array. The array was rotated around a vertical axis to twelve different positions during the tests thus providing a total of 84 measurement positions. The smaller measurement surface, which yielded the "near field" data, consisted of a rectangular box surrounding the source at a distance of one metre from the surface of the source. These measurements were recorded for a series of seventeen air compressors, one broad-band reference sound source and one enclosed loudspeaker excited by three different pure tone signals. The complete set of data from the compressors was used to compute sound power level for each of the sources to provide a test of the validity of near field measurements. The data were reprocessed using subsets of the near field data in order to evaluate the effects of sampling error. These results are used to provide a portion of the estimate of error of measurement, for various measurement methodologies.

\subsection{Measurement Procedures}

In this section we discuss the measurement site, the data acquisition and analysis instrumentation, the data reduction procedures and the detailed test procedures for taking "far field" and "near field" sound pressure level data. In the following, the term "far field" will be used without quotes to denote the $7 \mathrm{~m}$ radius test data, while "near field" will be similarly used to denote the measurement at $1 \mathrm{~m}$ from the source surface. In so doing, no claim is made or intended that these data are, in fact, in the acoustic far-field or near-field, respectively.

2.3.1. Measurement Site An agreement was reached with the U. S. Army for utilization of a hard surface test pad at Fort Belvoir, Virginia, for the data acquisition phase of this program. A plan view of the measurement site is shown in Figure 1. The test pad consisted of a $27 \mathrm{~m}$. diameter concrete surface, of roughly conical shape pitched to a drain (which was covered with a $6 \mathrm{~mm}$ thick steel plate throughout the tests) in the center. An estimate of the half-angle of the cone is $89.2^{\circ}$. An annular-shaped rolled clay area of about $60 \mathrm{~m}$ total diameter, surrounded the test pad, and provided increased clear area. This clay surface varied in elevation from 0 to $.3 \mathrm{~m}$ below the surface of the concrete test pad. The nearest major reflecting surfaces were a one-story corrugated steel building about $40 \mathrm{~m}$ northeast of the test pad, and the test equipment truck located about $50 \mathrm{~m}$ southeast of the test pad.

Other significant topographic details within a $75 \mathrm{~m}$ radius of the center of the test pad included a creek bed approximately $30 \mathrm{~m}$ south of the test site whose surface was 3 to 5 metres below the surface of the test pad, and a tree covered hill to the northwest of the test site which had a slope of $20-30^{\circ}$. The photographs in Figure 2 present views of the site from the south edge looking north and from the west edge looking east.

A pulse echo test, using the equipment shown in Figure 3, was used to quantitatively evaluate the effect of reflections. The worst case reflection, in the sense of poorest direct-to-reflected signal ratio, is that which returns to a point behind the major lobe of a directive source. A 10 in. diameter loudspeaker in an "infinite" baffle enclosure was used to simulate a directive source. Tone bursts at octave center frequencies from $125 \mathrm{~Hz}$ to $4 \mathrm{kHz}$ were used to investigate the strength of reflections in twelve directions at $30^{\circ}$ increments in angle around the test site. The one story building was found to produce significant mid and high frequency reflections, and was covered with a 3 in. glass fiber, building insulation, absorber (shown in Figure 2). With this modification, the strength of reflections was more than $15 \mathrm{~dB}$ below the direct signal at $180^{\circ}$ behind the loudspeaker (see Table 3.19 for directivity information on the loudspeaker at $500 \mathrm{~Hz}, 1000 \mathrm{~Hz}$ and $2000 \mathrm{~Hz}$ ). Since the 


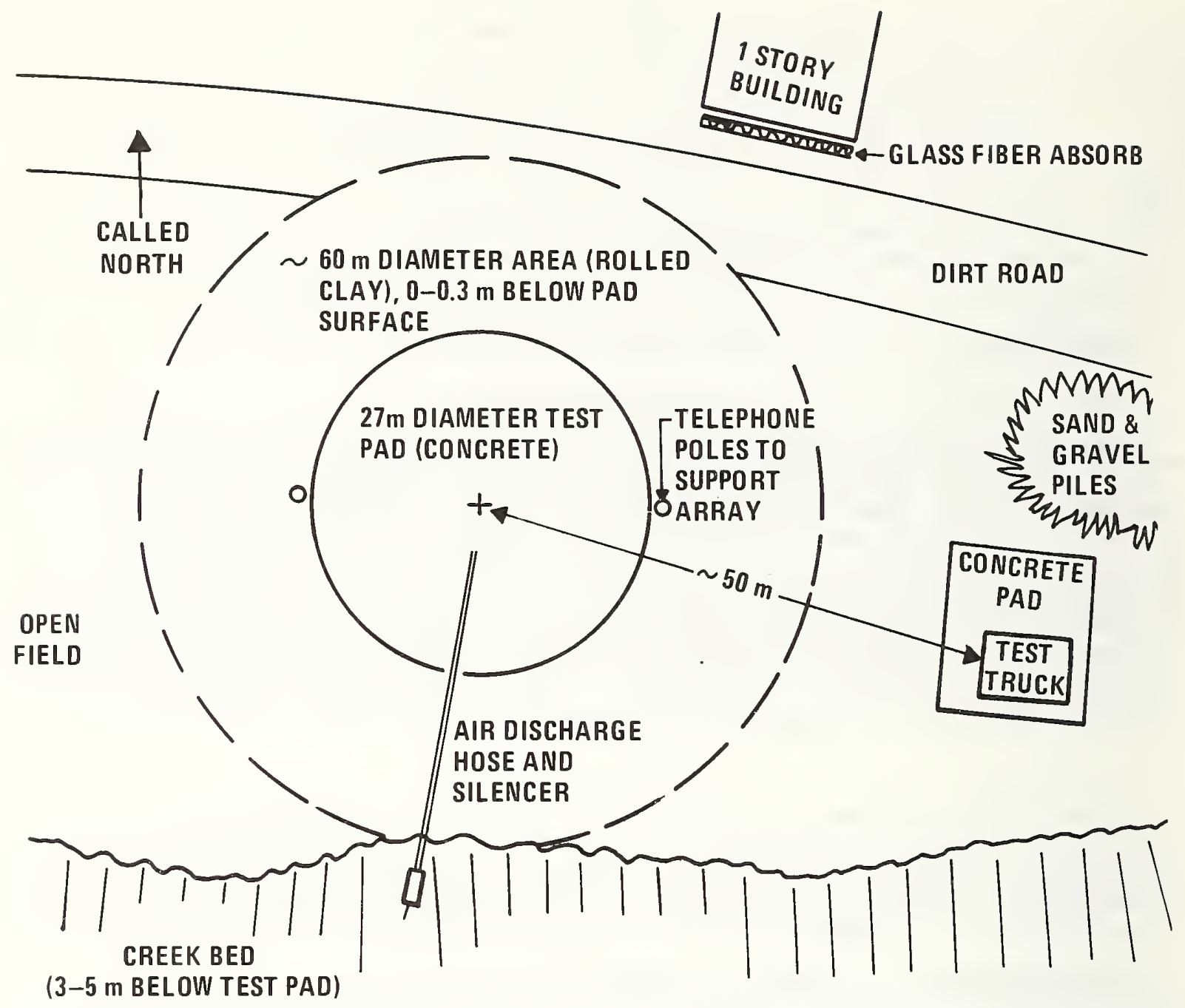

Figure 1 Field test site

directivity of the other test sources was found to be less than or equal to that of the loudspeaker, this leads us to state with confidence that the contribution of unwanted reflected signals was negligible at all test frequencies at all microphone positions.

The acoustic ambient of the test site was determined by three major sources -- steady traffic noise from a four-lane interstate highway approximately $1 \mathrm{~km}$ southeast of the site, aircraft and helicopter overflights from nearby airports, and rural fauna (principally birds and insects).

2.3.2 Instrumentation Figure 4 shows a schematic block diagram of the instrumentation used for the measurements reported here. The eight microphone channels each included a l-inch diameter Bruel \& Kjaer (B \& K) Type $4131^{-}$f condenser microphone cartridge with standard protection grid (fitted with a

$\underline{2 / \text { Commercial instruments }}$ are identified in this report in order to adequately specify the experimental procedure. In no case does such identification imply recommendation or endorsement by the National Bureau of Standards, nor does it imply that the equipment identified is necessarily the best available for the purpose. 


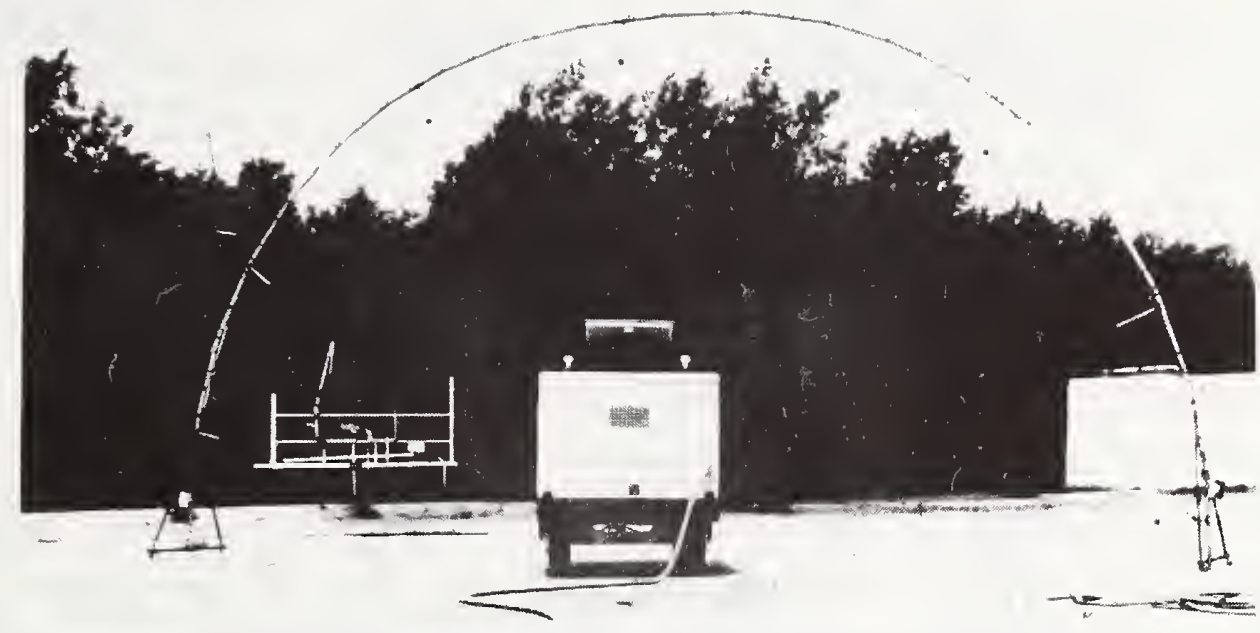

a) South edge looking north showing far field array in place (test no. 9)

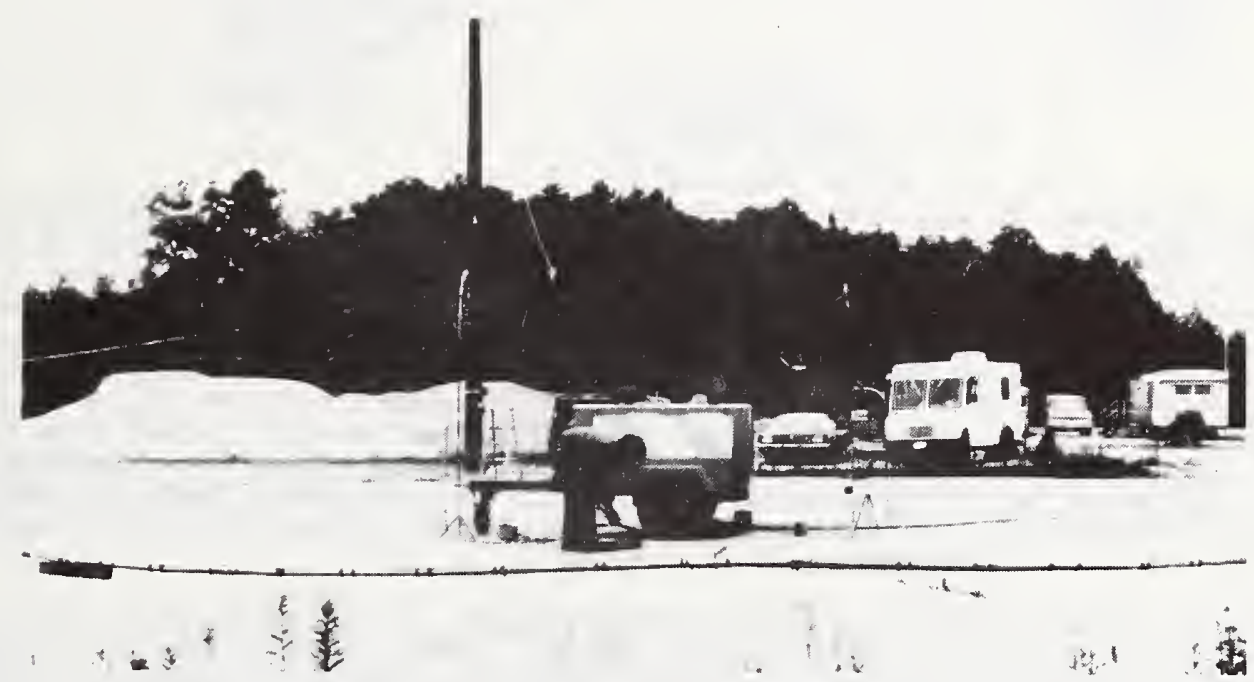

b) West edge looking east showing near field microphones in place (test No. 11) 


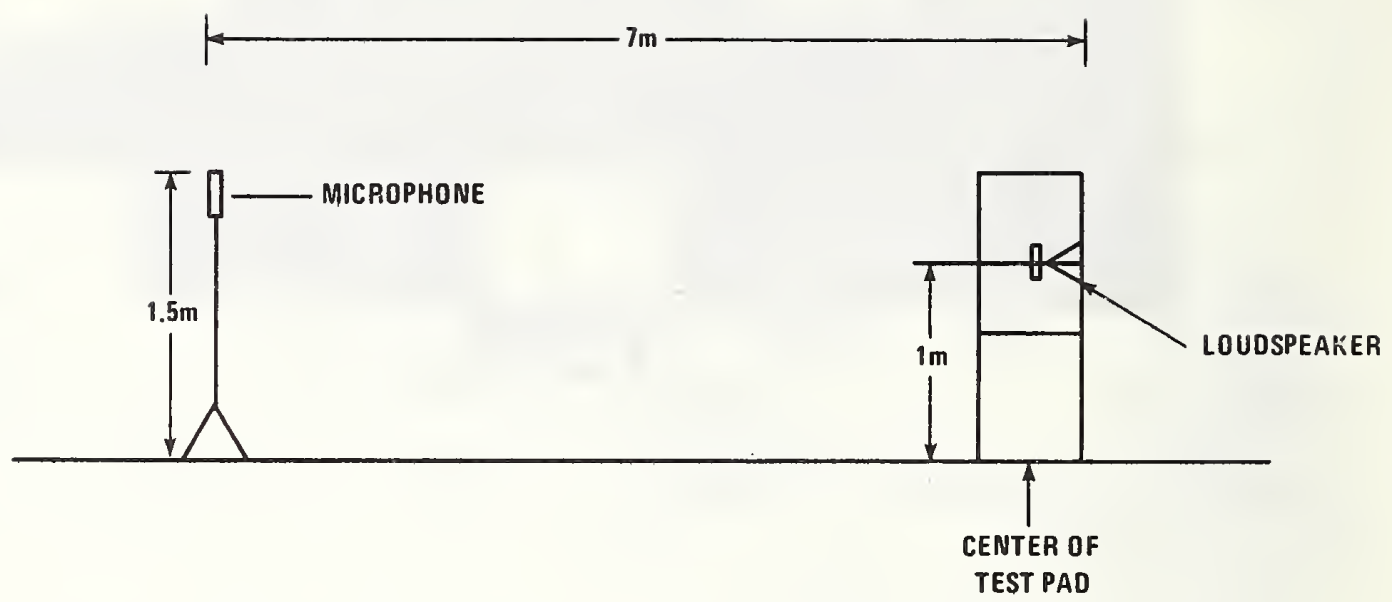

Figure 3 Equipment configuration for puise echo tests of . test site

desiccant dehumidifier to control humidity in the microphone cavity) and 10 cm diameter polyurethane foam windscreen, with B \& K Type 2619 FET cathode-follower preamplifier. Each pair of channels was driven by a battery-operated power supply. The signal from each channel was fed to a computer-controlled multiplexer (NBS designed and fabricated) which was used to electrically switch from one channel to another (over the frequency range $20 \mathrm{~Hz}-20 \mathrm{kHz}$, crosstalk between any two channels is greater than $-65 \mathrm{~dB}$, and channel gain is $0 \mathrm{~dB} \pm 0.2 \mathrm{~dB}$ ). The signal from the multiplexer was transmitted via coaxial cable to a B \& $K$ Type 3347 real-time one-third-octave band analyzer where the signal was analyzed in A-weighted, linear ( $2 \mathrm{~Hz}-20 \mathrm{kHz}$ ) and one-third octave bands from $12.5 \mathrm{~Hz}$ to $20 \mathrm{kHz}$. Output from the analyzer, in the form of digitally coded sound pressure levels, was sent on demand to a Raytheon type 704 minicomputer for manipulation and storage. Control of the computer was accomplished through an initial data acquisition and reduction program, with system operator interaction through a CRT terminal.

The signal being processed was continuously monitored, both audibly through a headset, and visually through the spectrum displayed on the analyzer. If (a) non-stationarity of spectra on a given channel, (b) significant level or spectrum change from channel to channel or, (c) non characteristic sound in the audible monitor was observed, the data processing was interrupted and the cause investigated.

Using these techniques, aircraft overflights were typically sensed prior to a visible identification, and stability and speed of the compressor in operation were monitored as well, thus permitting the operator to prevent processing of unwanted signals.

An eighth microphone was placed at a fixed height ( $1 \mathrm{~lm}$ ) and distance ( $4 \mathrm{~m}$ ) from the source for both the near and far field measurements. This position was used as a reference to verify constant source output throughout both tests.

Additional acoustic instrumentation included a sound level meter and octave band filter set for recording additional sound level data. Other instruments included a vane-type wind speed indicator, an optically-coupled tachometer for checking operating speed of the engine and fan, and a mercury column thermometer for monitoring test site temperature. 
B \& K 4145 MICROPHONE

CARTRIDGE, UA 0310

DEHUMIDIFIER。

2619 FET PREAMPLIFIER

B \& K 2804 POWER SUPPLY
NBS 8 CHANNEL COMPUTER CONTROLLED SWITCH (MULTIPLEXER)

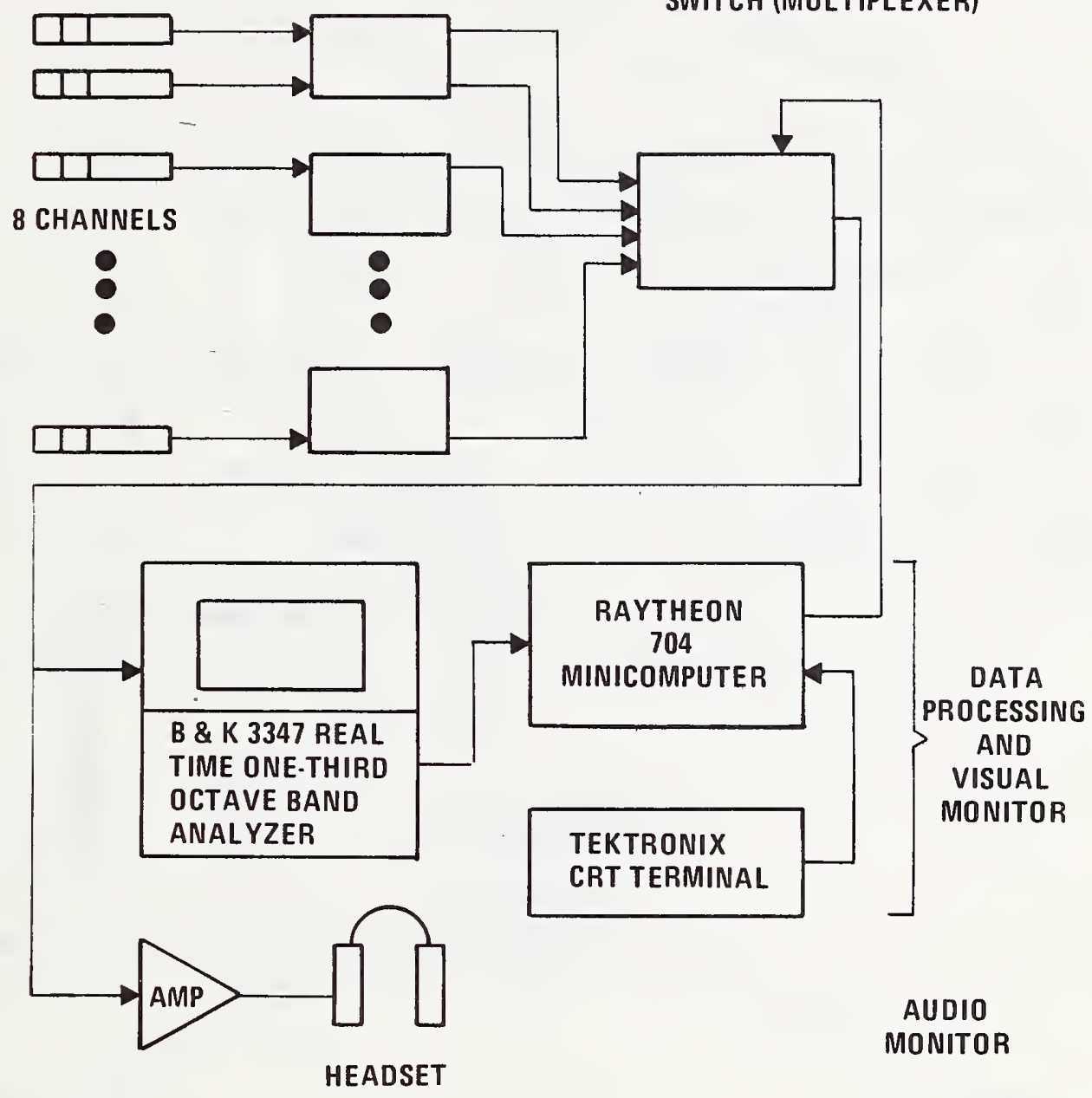

Figure 4 Schematic block diagram of data acquisition and analysis instrumentation

2.3.3 Test Plan The measurements on all sources were made according to the following test plan. Figure 5 shows the far field array, while Figure 6 shows a schematic illustration of the near field measurement positions referred to in the plan.

a) Assemble and check far field measurement array.

b) Load computer program and check.

c) Record frequency response of all channels for electrical pink noise input.

d) Record channel response to pistonphone calibration signal. If response between channels differs by more than $0.5 \mathrm{~dB}$ plus difference between microphone sensitivities, investigate problem

e) Erect far field array. 
f) Record first far field ambient noise for first array position (all channels).

g) Record far field data for source (12 array positions). During these runs, wind speed was monitored and no data recorded when speed exceeded $\sim 5 \mathrm{~m} / \mathrm{sec} .(12 \mathrm{mph}$ ). Signal was monitored and no data recorded when acoustic events occurred which were not represented in the ambient noise measurement (such as aircraft flyovers, etc.).

h) Record second far field ambient noise, for first array position.

i) Take down the array.

j) Record second pistonphone calibration.

k) Disassemble far field array and assemble near field array.

1) Record third pistonphone calibration.

m) Record first near field ambient noise.

n) Record near field data observing same limitations on wind and ambient noise as in g) above.

o) Record second near field ambient noise.

p) Record fourth pistonphone calibration.

q) Disassemble and store equipment.

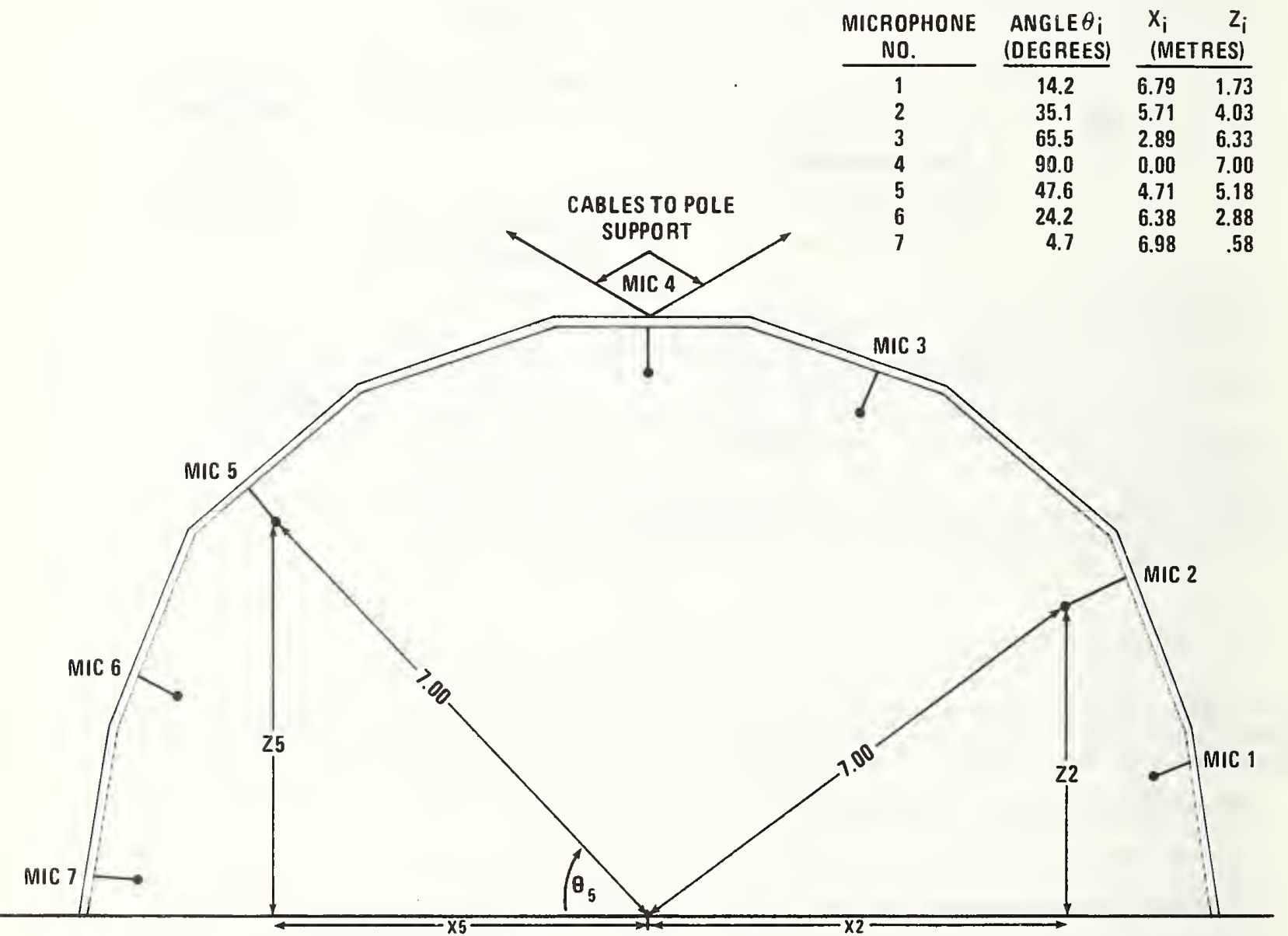

Figure 5 Far-field measurement array 


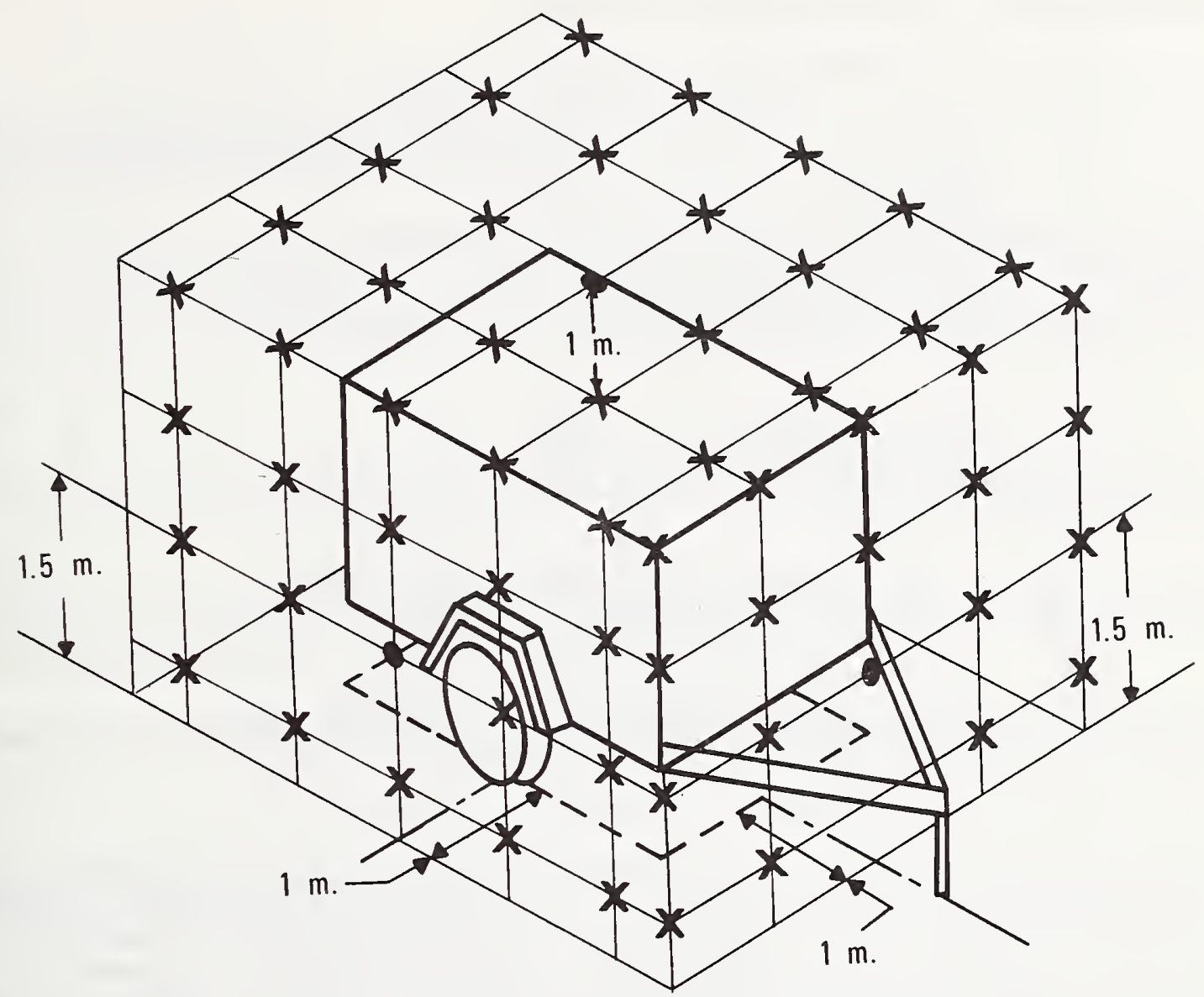

Figure 6 Representative near-field measurement positions

\subsubsection{Data Acquisition.}

The incorporation of a minicomputer into the data acquisition system permits considerable flexibility in manipulating information prior to storage, over that of the analyzer operating alone. The data acquisition program was structured to facilitate this. One major area where this facility was used was in the area of signal integration.

The digital coded signal available from the analyzer represents the R-C integrated sound pressure level rounded to the nearest $0.2 \mathrm{~dB}$. Three $\mathrm{R}-\mathrm{C}$ integration periods for the filters are provided in the analyzer, referred to by the manufacturer as "sine", "fast random" and "slow random", corresponding to nominally 0.2 second, 2.0 second and 20 second integration times. In the first two modes, the integration time constant is varied with frequency at low frequencies to maintain confidence levels of the same order of magnitude. For direct display of noise data extending to low frequencies, the "slow random" mode, because of its long integration time, should be selected in order to provide data with maximum precision. In order to obtain data which are not affected by startup transients, it is necessary to wait a period of 5 time constants after presenting the signal to the analyzer, prior to recording data. This implies that, per measurement, a total observation period of 120 to 140 seconds should be allowed for noise signals. Our measurement program included as many as 175 measurements per source, which would require about seven hours of observation time to complete. This situation forced the evaluation of alternate methods.

After some experimentation, the procedure finally selected involved using the "sine" time constant and summing repetitive samples ( 30 samples taken at one second intervals) to obtain an estimate of the average level. While performing this procedure, the temporal variance of the signal was also computed, permitting additional inquiries into the temporal "quality" of the signal. The 


$$
\begin{aligned}
p^{2}(i) & =\operatorname{Antilog}(\operatorname{SPL}(i) / 10) \\
\overline{p^{2}} & =\frac{1}{n} \sum_{i=1}^{n} p^{2}(i) \\
s_{p}^{2} & =\sum_{i=1}^{n}\left(p^{2}(i)-\overline{p^{2}}\right)^{2} /(n-1) \overline{\left(p^{2}\right)^{2}} \\
& =\frac{1}{(n-1)} \overline{\left(p^{2}\right)^{2}} \cdot \sum_{i=1}^{n}\left(p^{2}(i)\right)^{2}-\frac{n}{n-1}
\end{aligned}
$$

$$
\text { AVG SPL }=10 \log _{10}\left(\overline{p^{2}}\right)
$$

where SPL(i) is the ith sample of sound pressure level in a band

$$
\begin{aligned}
& \frac{p^{2}(i)}{p^{2}} \\
& s^{2} p
\end{aligned}
$$$$
\text { is the ith mean-square pressure in the band }
$$$$
\text { is the average mean-square pressure in the band }
$$

is the estimated temporal variance of mean-square pressure in the band normalized by the mean-square pressure squared.

AVG SPL is the average sound pressure level in the band.

The observation time is thus the number of samples taken times the time between samples. Allowing five seconds ( 5 time constants at low frequencies) prior to the start of data acquisition gives a total data acquisition time per measurement point of 35 seconds (considerably below that required by the "slow random" mode). Table 1 shows the results of some sampling tests using electrical pink noise as a source. The first line of the table shows the average standard error of the measured mean voltage level based on eight determinations of the mean using 30 samples per observation. The second line shows the pooled estimate of normalized temporal variance ( $s^{2}$ eq. 2.1 ) obtained in separate tests (total number of samples $=2800$ ). The third line shows the computed standard error of a determination of the mean from the temporal variance values using a propagation of error formulation; i.e.,

$$
\begin{aligned}
& \text { AVG SPL }=10 \log \sum_{i=1}^{n} \frac{p^{2}(i)}{n}=\frac{10}{\log _{e} 10} \log _{e} \overline{p^{2}} \\
& \begin{aligned}
\text { STD ERROR } & =\frac{10}{\log _{e} 10} \\
& =\frac{10}{\log _{e} 10}
\end{aligned}\left\{\frac{\frac{1}{n} \operatorname{VARIANCE~OF} \frac{\left(\overline{p^{2}}\right)}{n}}{\overline{\log ^{2}}}\right)^{1 / 2} \\
& =\frac{10}{2.303} \frac{s_{p}}{30} \simeq 0.78 s_{p}
\end{aligned}
$$

As might be expected, the standard deviation of levels computed from the mean values rounded to the nearest $0.1 \mathrm{~dB}$ is typically larger than that estimated from the temporal variance of the signal, since the latter values are known to a higher precision.

The values of line 3, Table 1.1, represent our current best estimate of the standard error for the sampling procedure for sound pressure level. However, because of roundoff error in the display of sound pressure level, the lower limit for standard error should be taken as 0.1 dB for A-weighted, linear, and $1.6 \mathrm{kHz}$ through $10 \mathrm{kHz}$ one-third octave band sound pressure levels.

No gain change corrections were made in the instrument system during data acquisition, but rather the overall system gain was determined from the pistonphone calibration. The precision of this calibration is estimated to be $+0.1 \mathrm{~dB}$ for comparison of relative levels between channels or runs. 
TARLE 1. INSTRUMENTATION DATA

1.1. Sampling Procedure Evaluation (see text, Section 2.2.4)

Pink Noise Excitation, "Sine" Time Constant, 30 Samples, 1 sec. apart. Eight Repetitions

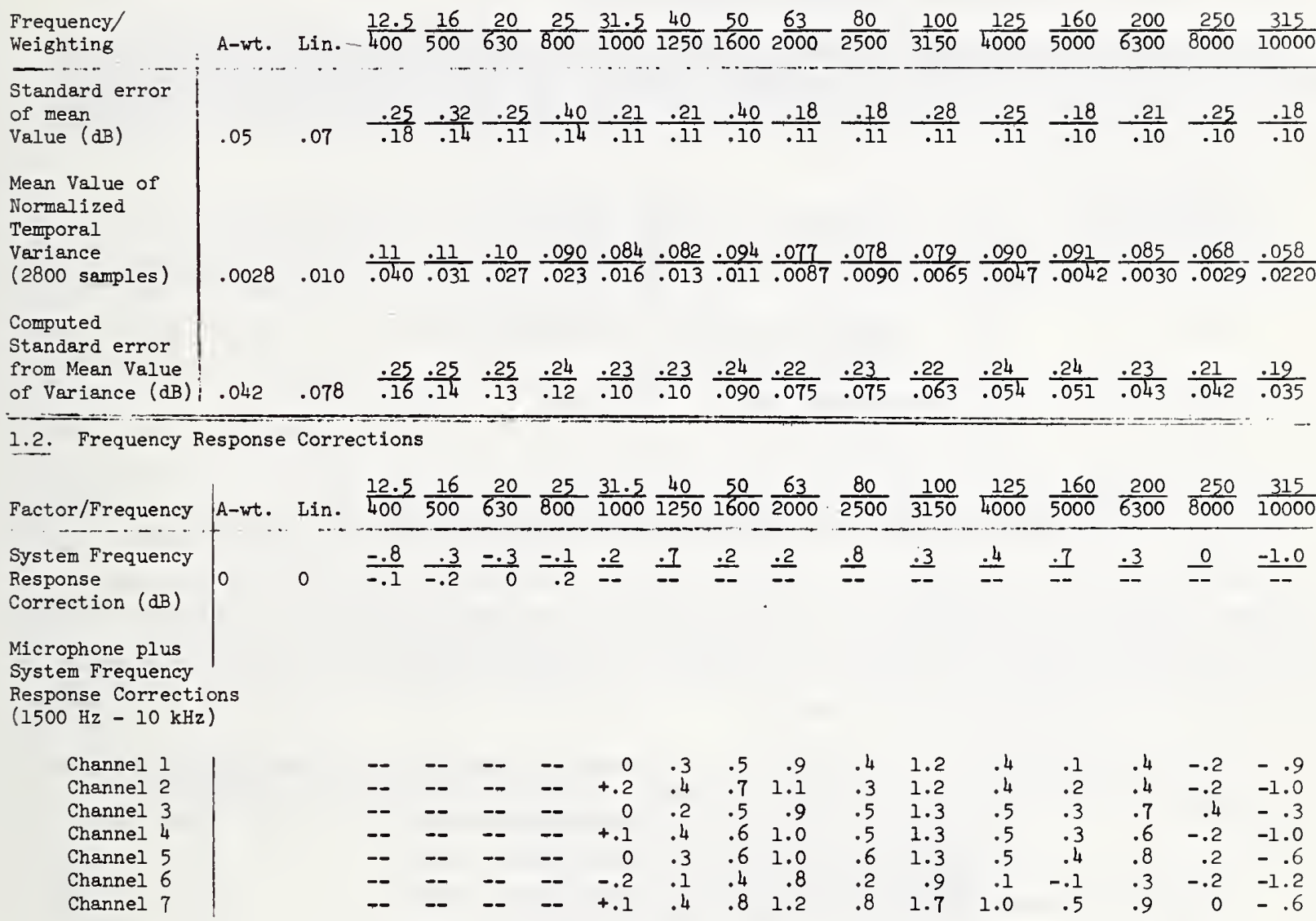

\subsubsection{Data Reduction and Corrections}

The raw data (consisting of indicated sound pressure levels) were corrected for four bias effects which included

1. Microphone cartridge frequency response

2. Measurement system gain calibration

3. Measurement system (other than microphone cartridge) frequency response

4. Influence of background noise.

The microphone cartridge frequency response was determined by measurement of the free field normal incidence frequency response (with windscreen in place) in the small NBS anechoic chamber. (The frequency response with the windscreen was significantly different from the frequency response without the windscreen. See Figure 7.)

The measurement system gain calibration was performed separately for each channel for both the far field and near field data sets. The value used was the average of the before and after pistonphone calibrations for measurement. The range of the before and after calibrations was 0.5 dB or less, with a typical value being $0.2 \mathrm{~dB}$ or less. 
The measurement system frequency response calibration was made using electrical pink noise excitation separately on each channel (flat spectrum within $\pm 0.1 \mathrm{~dB}$ in $1 / 10$ octave bands). This calibration, performed at the beginning and end of the measurement series, was found to be identical between channels within the accuracy of the calibration for the frequency range $12.5 \mathrm{~Hz}-2 \mathrm{kHz}$. The variations of frequency response above $2 \mathrm{kHz}$ were incorporated into the microphone frequency response correction. The calibration is based on 400 samples per channel, and is accurate to within \pm 0.1 dB. Table 1.2 provides the frequency response calibrations used.

Correction for the influence of background noise was made more difficult by the fact that values could not be simply deleted if in error, because of programming difficulties created in sound power level computations. As a result, the measured data were corrected and coded for validity according to the following scheme. The background noise used is the average of the before and after measurements.

\begin{tabular}{l|c|c}
$\begin{array}{l}\text { Difference between } \\
\text { signal and } \\
\text { background ( } \mathrm{aB})\end{array}$ & Code \\
\hline$>20$ & None & None \\
$20-3.0$ & $\begin{array}{l}\text { Standard Correction } \\
\text { to nearest } 0.1 \mathrm{~dB}\end{array}$ & None \\
$2.9-0.0$ & SPL $=$ SPL $-3 \mathrm{~dB}$ \\
$<0$ & SPL $=0$
\end{tabular}

For cases where the band level equaled the lower limit of the display scale, the level was also set to zero. Use of the coding will be further discussed under sound power level computations.

There was a need for a further correction to the near field data, due to the fact that the frequency response of a microphone to a sound pressure field at high frequencies is a function of the angle of incidence of the sound field. This is of little or no significance in the far field measurement since the angle subtended by the source at the measurement position is relatively small (half angle on the order of $15^{\circ}$ or less) so that, for microphones directed at the source, incidence perpendicular to the diaphragm can be assumed.

For the near field case, the microphone is not necessarily directed at the principal source, nor is the angle subtended by the source necessarily small. An expression which is appropriate for determining the true pressure if the distribution of intensity as a function of angle is known is

$$
p_{\text {true }}^{2}=p_{\text {measured }}^{2} \iint \frac{\int I(\theta, \phi) \sin \theta d \theta d \phi}{\int I(\theta, \phi) g^{2}(\theta) \sin \theta d \theta d \phi}
$$

where $I(\theta, \phi)$ is the scalar magnitude of the intensity at the angle $\theta, \phi$ from the normal to the microphone.

$g(\theta)$ is the microphone fractional response for plane wave incidence at the frequency $f$ and angle $\Theta$ from the normal to the microphone (assumed to be symmetric about the normal to the microphone), defined by the free field response of the microphone to a plane wave at the angle $\theta$.

$M(\theta)$ is the free field response of the microphone at the angle $\theta$.

Figure 7 provides a plot of -20 log $g(\theta)$ versus frequency. Two of the curves are manufacturer's data for the microphone cartridge with protecting grid and no windscreen. Two curves represent data measured at NBS on one of the microphones with a windscreen.

While it would be desirable to make a relatively exact correction for this effect, it is also clear that this requires much more detailed information than is available (such as distribution of intensity with angle as a function of frequency at each near-field microphone position for each compressor, and microphone directional characteristics throughout the range of angles). In lieu of this exact correction, and in order to place bounds on this error, let us try to find an approximate correction which might be applicable (in an average sense) to all the compressor data. One possible form for such a correction is to postulate a correction at an "equivalent angle of incidence" for the intensity, which is the same on the average for all microphones, defined by: 


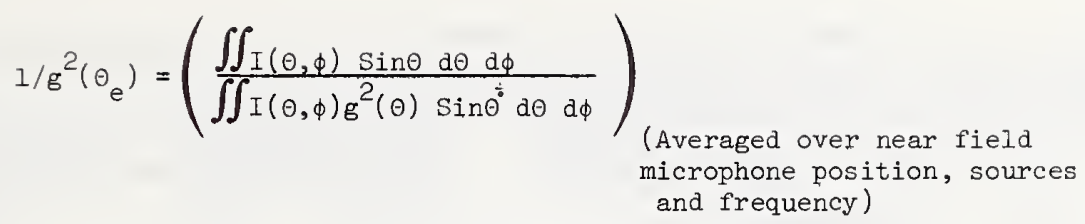

Under the assumptions of small angle ( such that $\sin ^{2} X \simeq x^{2}$ ), spherical source and measurement surface shape, uniform distribution of $I(\theta, \phi)$ over solid angle subtended by the source, microphone directed at the center of the source, and a weighting function of the form $g(\theta)=1 /(1-(\mathrm{ka} / 3) \mathrm{sin} \theta)(a=\mathrm{microphone}$ radius), which fits the data of Figure 7 up to $10 \mathrm{kHz}$ within $10 \%$, it can be shown that

$$
\theta_{\mathrm{e}}=\theta^{\prime} / 2
$$

where $\theta^{\prime}$ is the half-angle subtended by the source.

Since the average linear dimensions $(l, w, 2 h)$ of the sources are in the range of 2 to $2.5 \mathrm{~m}$ the subtended half-angle is in the range

$$
\arctan \left(\frac{2.0}{2} / 1\right) \leq \theta \leq \arctan \left(\frac{2.5}{2} / 1\right)
$$

or

$$
\begin{aligned}
& 45^{\circ} \leq \theta \leq 55^{\circ} \\
& 30^{\circ} \leq \theta_{e} \leq 40^{\circ}
\end{aligned}
$$

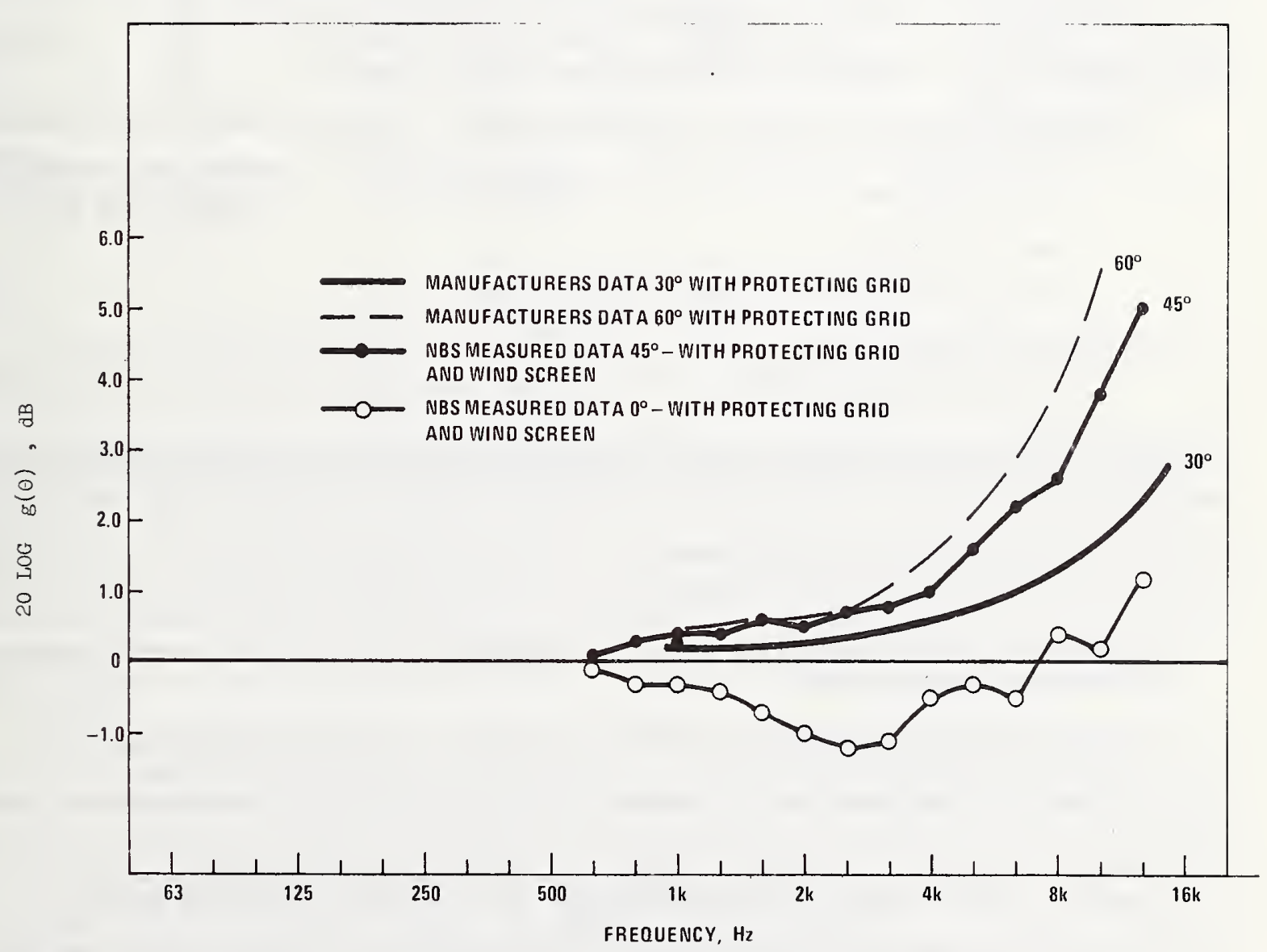

Figure 7 Microphone correction factor (Type 4145 cartridge) 
Thus when comparing near and far field sound power level data we urge that the near-field data be corrected by the amount shown in Figure 7 using the curve for $\theta=30^{\circ}$. This correction will not be applied to the data as presented as sound power data on an individual machine.

In passing, we note that using the same figures for a 7 metre microphone position, we find

$$
\theta_{e}(\text { far-field })<7^{\circ}
$$

and using the analytic form of $g(\Theta)$ given above

$$
-20 \log g\left(\theta_{e}\right) \leq 0.1 \mathrm{~dB}
$$

throughout the frequency range of interest.

We further note that this problem can be minimized by using a smaller microphone. With a half-inch microphone, for instance, the curves for $g(\theta)$ are shifted one octave higher in frequency, so that the estimated value of the correction would be less than about $1.0 \mathrm{~dB}$ at the highest test frequency. Unfortunately, such microphones (with dehumidifiers) were unavailable during these tests.

\subsection{Description of Compressor Sample}

Table 2 provides descriptions of the pertinent parameters of the individual sources tested.

In terms of the relevant acoustic parameters, the sample was intended to complement tests by others[4]. The total compressor sample (including these and other tests) is a sample reflecting several factors according to current economic data on the industry as previously compiled[5]. The factors which determined the relative number of compressors in the sample were as follows:

a) Manufacturer: weighted by estimated share of air compressor market.

b) Compressor type: weighted by estimated number of units produced.

c) Compressor size and power source: weighted according to estimated number of units produced in each of five ranges:

$$
\begin{aligned}
& \text { 1. gas, } 75-124 \mathrm{cfm} \\
& \text { 2. gas, } 125-250 \mathrm{cfm} \\
& \text { 3. diesel, } 125-249 \mathrm{cfm} \\
& \text { 4. diesel, } 250-500 \mathrm{cfm} \\
& \text { 5. diesel, over } 500 \mathrm{cfm} \text {. }
\end{aligned}
$$

Our portion of the sample consisted of smaller size machines which were more readily transportable to a common test site while other tests on larger machines were more conveniently tested at the site of manufacture.

The test sample has the following parameters.

a) A total of 17 compressors.

b) Three reciprocating compressors of capacity 100-200 cfm -- one gasoline-powered engine, two diesel-powered, none quieted.

c) Seven rotary screw compressors of capacity $85-185$ cfm -- five gasoline-powered, two diesel powered, four quieted.

d) Seven rotary vane compressors of capacity 125-900 cfm -- three gasoline-powered, four diesel powered, four quieted.

e) Seven manufacturers represented; nine gasoline engine powered; eight diesel-powered; eight standard, while nine were quieted by the manufacturer; total capacity range 85-900 cfm.

All compressors were obtained through rental in the Washington, D. C. metropolitan area, and were tested as received. Age of the machines varied from new to 1400 hours, with most in the range of less than 500 hours. No special preparation by the manufacturer was made that we are aware of. 


\begin{tabular}{|c|c|c|c|}
\hline $\begin{array}{l}\text { Test } \\
\text { No. }\end{array}$ & $\begin{array}{l}\text { Nominal Com- } \\
\text { pressor/Vol- } \\
\text { ume Flow/ } \\
\text { Rated } \\
\text { Pressure } \\
\text { (cfm/psi) }\end{array}$ & Type* & $\begin{array}{l}\text { Engine } \\
\text { Type** } \\
\text { No. of } \\
\text { Cyl. } \\
\end{array}$ \\
\hline 1 & $185 / 100$ & $S$ & $6 / 4$ \\
\hline 2 & $150 / 100$ & $\mathrm{R}$ & $G / 2$ \\
\hline 3 & $160 / 100$ & V & $\mathrm{D} / \mathrm{NR}$ \\
\hline 4 & $150 / 100$ & $\mathrm{~S}$ & $D / 4$ \\
\hline 5 & $200 / 100$ & $R$ & $D / 4$ \\
\hline 6 & $100 / 100$ & $\mathrm{R}$ & $D / 2$ \\
\hline 7 & $160 / 100$ & V & G/4 \\
\hline 8 & $125 / 100$ & V & $G / 4$ \\
\hline 9 & $365 / 100$ & V & $D / 4$ \\
\hline 10 & $900 / 125$ & V & $D / 8$ \\
\hline 11 & $100 / 100$ & $\mathrm{~S}$ & $G / 4$ \\
\hline 12 & $175 / 100$ & $\mathrm{~S}$ & $G / 4$ \\
\hline 13 & $175 / 100$ & $\mathrm{~s}$ & $\mathrm{D} / 4$ \\
\hline 14 & $185 / 100$ & $S$ & $D / 4$ \\
\hline 15 & $175 / 100$ & $S$ & $G / 4$ \\
\hline 16 & $85 / 100$ & $\mathrm{~s}$ & $G / 4$ \\
\hline 17 & $150 / 100$ & $\mathrm{~S}$ & $G / 4$ \\
\hline 18 & NAt & NA & NA \\
\hline 19 & NA & NA & NA \\
\hline & & & \\
\hline & & & \\
\hline
\end{tabular}

* R=Reciprocating compressor $\mathrm{S}=\mathrm{Rotary}$ screw compressor $\mathrm{V}=$ Rotary vane compressor
Size of Enclosure ( $\mathrm{L}, \mathrm{W}, \mathrm{H}$ in metres) (1)

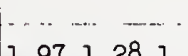
$1.97,1.28,1.4$
$1.73,177,1.47$ $2.07,1.14,1.772350$ $1.71,1.24,1.401950$ $1.83, .98,1.50 \quad 1750$ $1.52, .67,1.42 \quad 1650$ $2.16,1.28,1.782250$ $1.78,1.30,1.851850$ $3.66,1.82,2.141800$ $4.29,2.19,2.512100$ $1.78,1.22,1.372150$ $2.70,1.29,1.432300$ $2.70,1.29,1.432300: 153$ $2.70,1.29,1.43220073$ $1.99,1.27,1.45 \mid 2150: 72$ $1.96,1.10,1.34: 2000: 66$ $1.93,1.24,1.363000 \quad 100$ .35 dia $x \cdot 7,1750$ NA<smiles>[Mg][AsH2][AsH2]</smiles>

** $G=$ Gasoline engine $\mathrm{D}=\mathrm{Diesel}$ engine

\begin{tabular}{|c|c|c|c|c|}
\hline $\begin{array}{l}\text { Engine } \\
\text { Firing } \\
\text { Freq. } \\
(\mathrm{Hz})\end{array}$ & $\begin{array}{l}\text { Cooling } \\
\text { Fan } \\
\text { Blade } \\
\text { Passage } \\
\text { Freq. } \\
(\mathrm{Hz})\end{array}$ & $\begin{array}{l}\text { Usage } \\
\text { at } \\
\text { Start } \\
\text { of } \\
\text { Test } \\
\text { (Hours) }\end{array}$ & $\begin{array}{l}\text { Stan- } \\
\text { dard } \\
\text { Vs. } \\
\text { Quieted }\end{array}$ & Notes \\
\hline 78 & Est.240 & NR & $\mathrm{S}$ & \\
\hline 38 & Est.240 & 1011 & $\mathrm{~S}$ & 4 cyl. in-line block \\
\hline NRt+ & 300 & NR & $Q$ & \\
\hline 130 & 300 & 547 & $\mathrm{~S}$ & \\
\hline 116 & 210 & 6.1 & $\mathrm{~S}$ & $8 \mathrm{cyl}$. V-8 block \\
\hline 55 & 150 & NR & $\mathrm{S}$ & 4 cyl. in-line block \\
\hline 75 & 225 & 2.3 & $Q$ & \\
\hline 62 & 250 & 14.5 & $Q$ & \\
\hline 120 & 1267 & 11.8 & $Q$ & 20 blade fan@ $3800 \mathrm{rpm}$ \\
\hline 280 & 307 & 1.0 & $Q$ & \\
\hline 72 & 250 & 1440 & S & \\
\hline 74 & 235 & 1086 & $Q$ & \\
\hline 153 & 250 & $\mathrm{NR}$ & $Q$ & \\
\hline 73 & 280 & 776 & $\mathrm{~S}$ & $\begin{array}{l}\text { Governed at less than } \\
\text { rated speed }\end{array}$ \\
\hline 72 & 250 & 398 & $\mathrm{~S}$ & \\
\hline 66 & 230 & 1300 & S & \\
\hline 100 & 300 & 297 & $S$ & \\
\hline NA & NR & -- & - & $\begin{array}{l}\text { Broad band reference } \\
\text { sound source }\end{array}$ \\
\hline NA & NA & $-\infty$ & -- & $\begin{array}{l}.25 \mathrm{~m} \text { dia. loudspeaker } \\
\text { (tone source) center- } \\
\text { line of speaker } 1 \mathrm{~m} \\
\text { above ground }\end{array}$ \\
\hline
\end{tabular}

$+N A=$ Not applicable $t+N R=$ Not recorded

\subsection{Compressor Operation}

The major problems associated with compressor operation for these tests were providing for acoustically controlled discharge of the compressed air, to assure insignificant contribution to the measured noise, and ensuring constancy of operation at rated capacity.

Discharge air from the compressor was fed through a $30 \mathrm{~m}$ length of commercial high pressure rubber hose to a commercial automobile muffler, where it was discharged to the atmosphere. The muffler was placed in the creek bed (see Figure 2) so that the bank of the creek would provide shielding of the discharge noise from the test site. Measurements of octave band sound pressure levels near the discharge were made for each test, and extrapolations of the measured levels, assuming hemispherical spreading and ignoring shielding, indicated that the discharge noise from the muffler was more than $10 \mathrm{~dB}$ below the compressor noise at all measurement locations. In some cases more than one hose line and silencer had to be used to accommodate the compressor volume flow. For the two larger machines (tests 9 and 10) a blow-down silencer of undetermined manufacture (obtained from the rental source), and large diameter hose was used in place of the above air discharge silencing arrangement.

The operating point for the test was the condition of the compressor supplying rated flow. This condition occurs when the compressor is operated at rated speed and pressure. Establishment of this point was made simpler by the fact that most of the compressors had an engine speed governor which operated on the difference between receiving tank pressure and rated pressure. Rated speed was thus obtained by throttling the flow of discharge air until the compressor held a constant receiving tank pressure near rated pressure (as indicated on the compressor air pressure gauge) and verifying that the engine was operating at rated speed (as measured by an independent tachometer). At this point, a reduction in air flow will be followed by a reduction in engine speed (for a proportional controller), while an increase in air flow will be followed by a reduction in receiving tank pressure. This operating point was easily established once the compressor had been operated for 15-20 minutes and conditions approached a thermal steady-state.

Once at this point, engine speed could be monitored within about +50 rpm by inspection of the displayed noise spectrum at bands near the engine firing rate frequency. Since the firing rate tone 
typically excited one or the other of the adjacent bands in addition to the band containing the tone, the difference in decibels between these two bands could be taken as a sensitive indicator of firing rate frequercy. This procedure can not be used if the spectrum of broad band noise is within lodB or so of the indicated tone level in each band. In this case, engine speed was monitored audibly, with frequent tachometer checks.

\subsection{Measurement Locations and A-Weighted Sound Level Data}

\subsubsection{Far Field Measurements}

The far field sound pressure level data were taken using the array shown in Figure 5 (also visible in Figure $2 a$ ). The seven microphones were located on nominal one-half metre long standoffs from a semi-circular arc constructed of steel pipe and tubing. The arc was supported from above by two cables from the arc center to poles located on the east and west edge of the test pad. The ends of the arc were supported on casters to facilitate rotation. Positioning was accomplished by pins at the end of the are which fit into holes drilled in the concrete test pad. The maximum radial positioning error of a microphone in the array is estimated from sample measurements of positions to be less than $\pm 0.1 \mathrm{~m}(1.4 \%)$ including all array positioning effects such as changing arc shape from change in proportion of weight supported by overhead cables. Angular positioning error is estimated to be less than $\pm 1^{\circ}$ in both azimuthal and polar angles. In terms of inverse square spreading, the radial error translates into a possible error in sound pressure level estimation of less than +0.1 $\mathrm{dB}$, per observation. Since the principal source of radial error arises from change of shape of the arc -- which leads to positive errors at some positions while there are negative errors at other positions -- this source of error is believed to average out in the estimate of power rather than produce a systematic bias. The angular positioning error leads to random sampling on the hemispherical surface (i.e., imprecision in directivity) as opposed to a systematic bias in sound power determination

In addition to these array data, octave-band sound pressure level data were also taken using a hand-held sound level meter employing a modified form of the present industry methodology[6]. The intent of the NBS Far Field methodology initially recommended to EPA was to provide an upper bound estimate of sound power level based on measurements made at six locations. The six locations include:

1. Four positions perpendicular to the center of each side of the

compressor at a distance of 7 metres from the center of the compressor (data taken at the elevation in the range 0.8 to $1.6 \mathrm{~m}$ which yields the highest A-weighted sound level).

2. One location at an elevation of 1 metre above the ground, on the 7 metre radius circle centered on the machine, at the location giving the maximum A-weighted sound level.

3. One location directly above the center of the compressor, at a height of seven metres above the gound plane (data from microphone four of the array was used for this location).

The data, using this procedure, were taken in the same time interval as the data from the far-field array, and are reported here as "Far-Field Methodology" data. Far-field array and methodology data were recorded for the seventeen compressors, and also two known sources, to investigate the effectiveness of the far field test procedures for these sources. The first of these was a broad band "reference sound source" consisting of an electric motor-driven centrifugal fan with cylindrical symmetry, which is nominally omnidirectional. The second source was a $0.25 \mathrm{~m}$ diameter loudspeaker, mounted in a $0.4 \mathrm{~m}$ cubical sealed baffle. The baffle was located at the center of the test pad with the loudspeaker axis horizontal, $1 \mathrm{~m}$ above the test pad, pointed at approximately 220 degrees from north. The speaker was driven in different tests with tones of $500 \mathrm{~Hz}, 1 \mathrm{kHz}$ and $2 \mathrm{kHz}$. The principal purpose of this test was to give example data of the measurement problem associated with tones.

A tabulation of the A-weighted far-field sound level data is given in Tables 3.1 to 3.19. The "Far-Field Data" are given in tabular form in the form of a directivity pattern, with columns corresponding to azimuthal angle from $0^{\circ}$ to $330^{\circ}$ from north (compressors were aligned on the test pad with the tow bar pointing north and sides oriented in the north, east, south and west directions). The "Far-Field Methodology" data are given in tabular form underneath these data. Also given is the A-weighted sound power level computed from the far-field and "far-field methodology" data, for reference purposes (see section 3 for calculation procedures). The sound level corresponding to the average mean square pressure over the hemisphere (frequently referred to as the "energy average") may be calculated from the sound power level according to:

$$
L_{p}(r)=L_{W}-10 \log 2 \pi r^{2}
$$

It is power level, $\mathrm{aB}$ re $10^{-12}$ watt

$L^{W}$ is the sound pressure level, $\partial B$ re $2.10^{-5}$ pascal

$r^{p}$ is the radius of the hemisphere, metres

A nominal value of impedence equal to $400 \mathrm{mks}$ rayls is assumed. 
At seven metre radius, the average A-weighted sound level thus is

$$
L_{p A}(7 m)=I_{W A}-24.9 \mathrm{~dB}
$$

\subsubsection{Near Field Measurements}

The near-field data were taken using seven microphone channels with six of the microphones mounted on tripods, and the seventh suspended from a "skyhook" formed by the support cables used to position the far-field microphone array. Figure 6 shows representative measurement positions on a measurement surface. The microphone positions were determined according to the following rules.

1. The measurement surface was a rectangular box of dimensions

I $x W \times H$ where $I=\ell+2, W=W+2, H=h+l$ and where $\ell, w$ and $h$ are the length width and height of the compressor excluding tow bar, tires and fenders, and other small projections with linear dimensions less than 0.5 metres (such as exhaust pipes, etc.). The four vertical plane surfaces were located at distances of $+\mathrm{L} / 2$ and $+W / 2$ from the geometric center of the compressor, and perpendicular to the longitudinal and Iateral centerlines. The horizontal plane surface was located at the distance $H$ above the reflecting plane. These surfaces are nominally 1 metre from the surface of a compressor with a rectangular enclosure.

2. The microphone positions on the measurement surface were located on a 1 x 1 metre square grid. The grids were located on the vertical sides so that a measurement position was on the center of each side at a height of 1.5 metres. This grid location yields measurements positions at heights of $0.5,1.5,2.5 \mathrm{~m}$, above the ground plane, spaced $1 \mathrm{~m}$ apart in either direction from the center of that side. The grid on the horizontal measurement surface was centered in the center of that surface. For all compressors the measurement positions near the engine exhaust were displaced along a grid line to the closest point 1 metre from the end of the exhaust pipe.

3. Microphones were oriented with the plane of the microphone diaphragm in the plane of the measurement surface.

4. Near edges or corners where pairs of measurement locations from adjacent surfaces were less than $0.25 \mathrm{~m}$ apart, one of the pair (usually on the vertical surface) was deleted.

The rectangular measurement surface was chosen because it was the only surface being considered by Iso working groups on sound power measurement standards, at the time of the tests (May-July, 1974).

The lower half of Tables 3.1-3.18 titled "near-field data presentation" presents the A-weighted sound level data from these tests in a format which facilitates relating level and position. Note that levels measured closest to the ground plane are furthest from the center of the table. 
Table 3.1

A-WEIGHTED SOUND PRESSURE LEVEL DATA

Test Number 1 Compressor Output: $185 \mathrm{cfm}$

Engine/Compressor Type: Rotary screw, Gab, icanadica

Compressor Size: $1.97 \times 1.28 \times 1.4 \mathrm{~m}$

* far fielo data ( 7 metre radius)

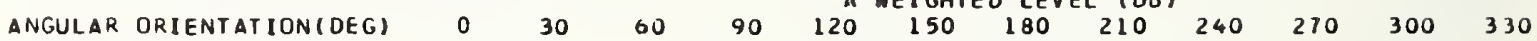
HIC HEIGHT NUM ELEVATION

(METRFS) (OEG)

$\begin{array}{rrrrrrrrrrrrrrrr}-575 & 7 & 4.7 & 80.4 & 81.5 & 82.8 & 80.6 & 81.0 & 84.9 & 83.0 & 84.5 & 82.4 & 78.8 & 79.0 & 80.3 \\ 1.725 & 1 & 14.4 & 78.4 & 79.3 & 79.8 & 80.9 & 80.8 & 82.4 & 81.5 & 82.9 & 80.6 & 77.2 & 77.8 & 79.4\end{array}$

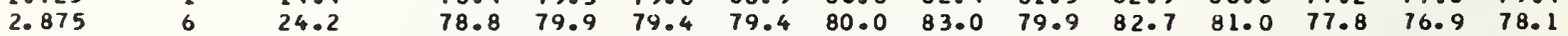

$\begin{array}{llllllllllllllll}4.025 & 2 & 35.2 & 79.5 & 80.0 & 80.2 & 79.5 & 80.2 & 82.5 & 81.3 & 83.1 & 81.5 & 78.4 & 77.2 & 79.1\end{array}$

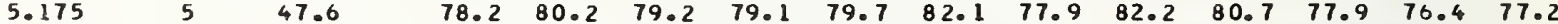

$\begin{array}{llllllllllllllll}6.375 & 3 & 65.7 & 77.3 & 77.6 & 78.0 & 78.4 & 79.5 & 80.7 & 80.3 & 80.7 & 78.4 & 77.4 & 76.5 & 77.0\end{array}$

$\begin{array}{llllllllllllllll}7.000 & 4 & 90.0 & 76.2 & 76.5 & 76.4 & 76.6 & 76.1 & 76.2 & 75.8 & 76.6 & 76.0 & 75.9 & 76.0 & 76.5\end{array}$

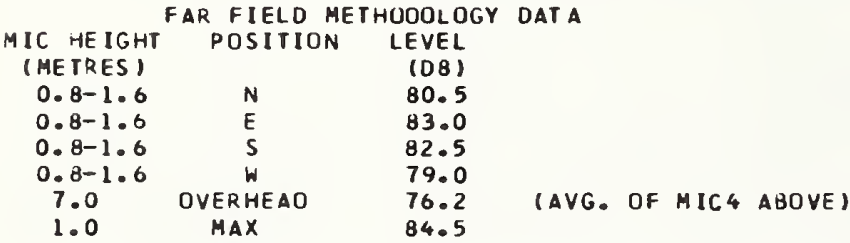

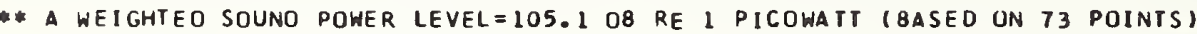

JUL IAN DAY 144 TEST NUMBER 1

NEAR FIELD DATA PRESENTATION

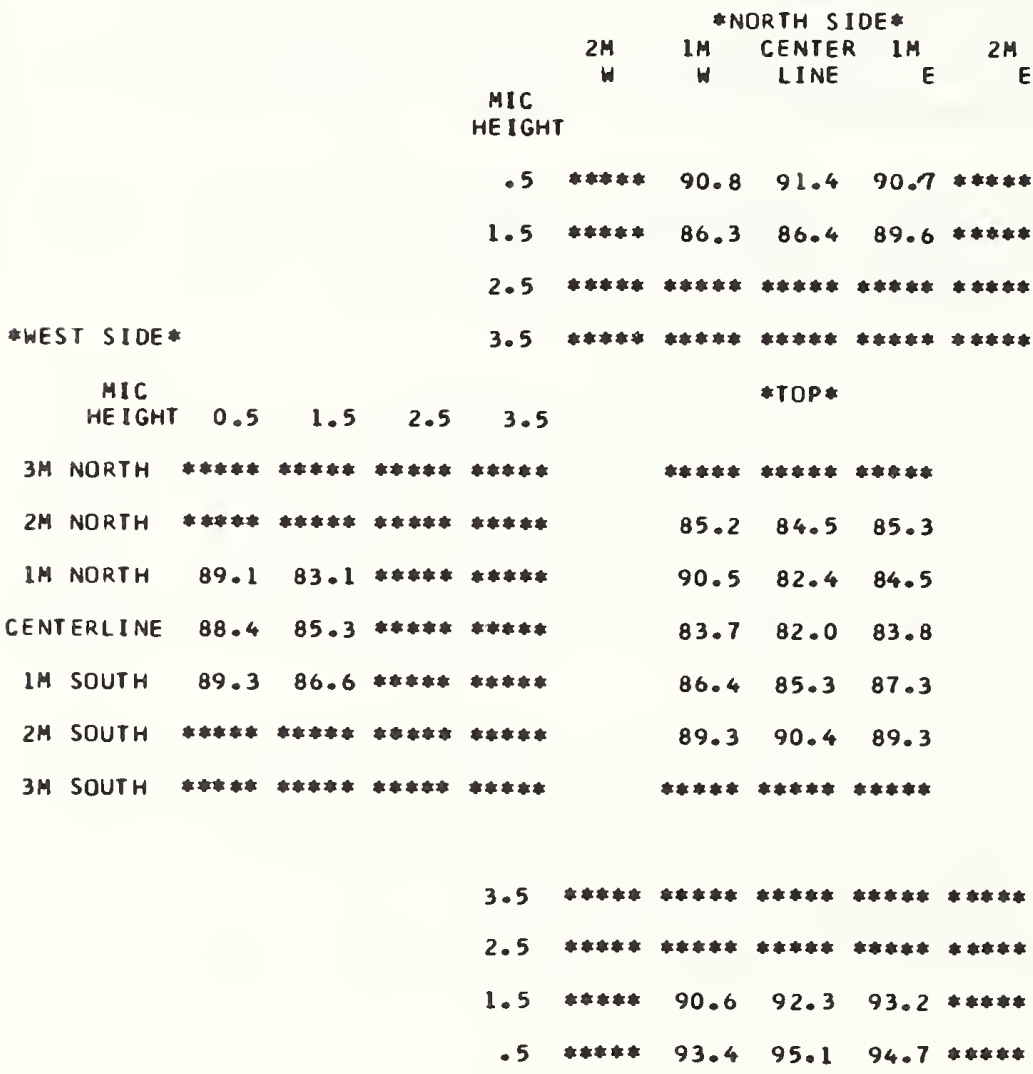

SOUTH SIDE* 
Table 3.2

A-WEIGHTED SOUND PRESSURE LEVEI DATA

Test Number 2 Compressor Output: $150 \mathrm{cfm}$

Engine/Compressor Type:Reciprocating, Gas, Standard

Compressor Size: $1.73 \times .77 \times 1.47 \mathrm{~m}$

** FAK FIELD DATA ( 7 METRE RADIUS)

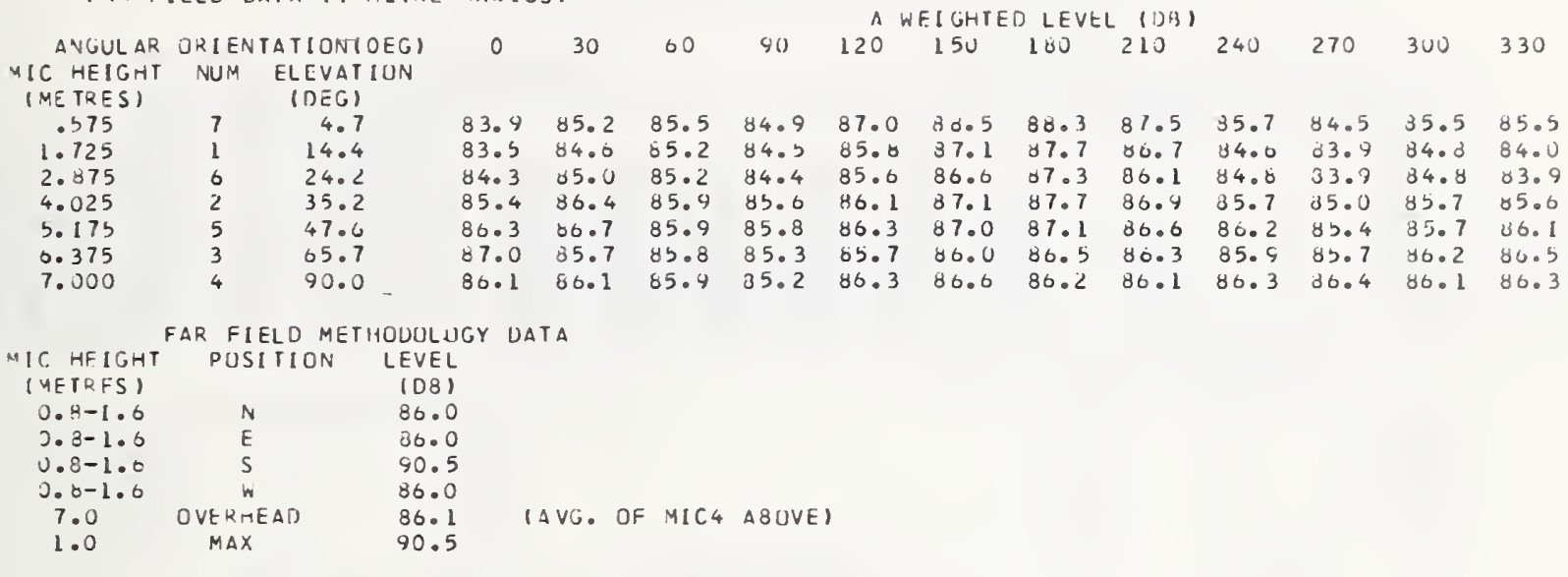

* A WEIGHTEU SUUND POWER LEVEL=110.8 DB RE 1 PICOWATT (8ASEU ON 73 POINTS)

JULIAN DAY 155 TEST NUMBER 2

NEAR FIELD DATA PEESENTATION

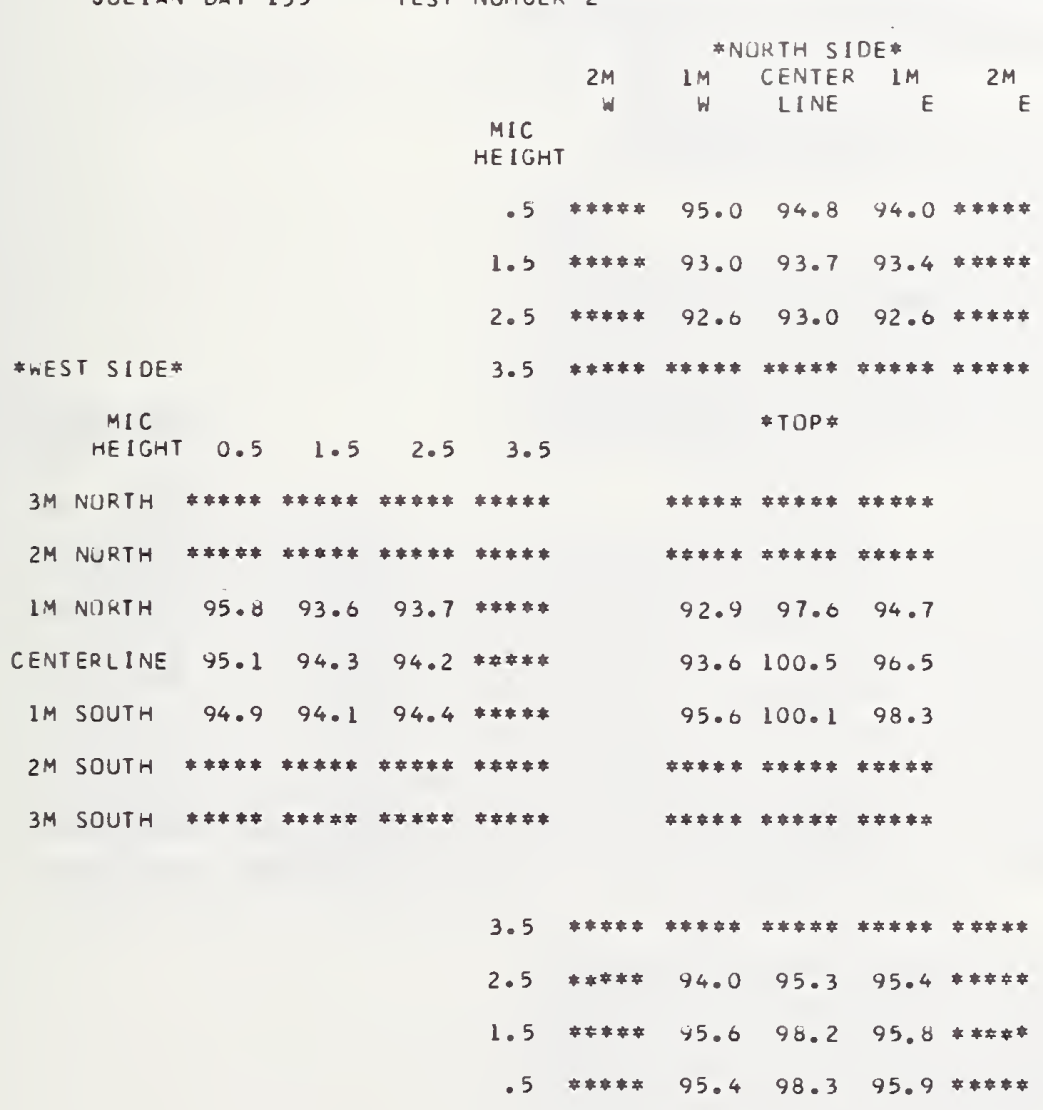

SUUTH SIDE\# 
Table 3.3

A-WEIOHTED SOUND PRESSURE LEVEL DATA

Test Number 3 Compressor Output: $160 \mathrm{cfm}$

Eng1re/Compressor Type: Rotary vane, D1esel, Quieted

Compressor S1ze: $2.07 \times 1.14 \times 1.77 \mathrm{~m}$

\begin{tabular}{|c|c|c|c|c|c|c|c|c|c|c|c|c|c|c|}
\hline$\triangle N G U L A R$ & UसIENI & AT IUN $(D E C B)$ & 0 & 30 & 60 & 90 & 120 & 150 & 180 & 210 & 240 & 270 & 300 & 310 \\
\hline 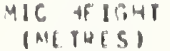 & NUM & $\begin{array}{l}\text { ELEVAYIUN } \\
\text { (I)P(i) }\end{array}$ & & & & & & & & & & & & \\
\hline 1.725 & 1 & 14.4 & 01.7 & 42.3 & 81.7 & 80.2 & 81.2 & 02.0 & 82.3 & 32.2 & 80.7 & 80.7 & 79.5 & $7+1.4$ \\
\hline 2.0175 & 6 & 24.2 & $\theta 1.9$ & UL. & 80.9 & 80.3 & 81.8 & 83.2 & 83.7 & 82.0 & 82.3 & 81.3 & 30.3 & 01.0 \\
\hline 4.1125 & 2 & $3, .2$ & 30.8 & 70.7 & 01.00 & 30.4 & 82.0 & 62.2 & 80.0 & 82.3 & 32.2 & 80.7 & 8ง. 7 & 80.9 \\
\hline 3.173 & 5 & 47.6 & 83.2 & 92.7 & 82.5 & 40.9 & $\Delta 1 . y$ & 82.7 & 83.0 & 83.1 & 83.2 & 01.7 & 32.1 & 02.8 \\
\hline
\end{tabular}

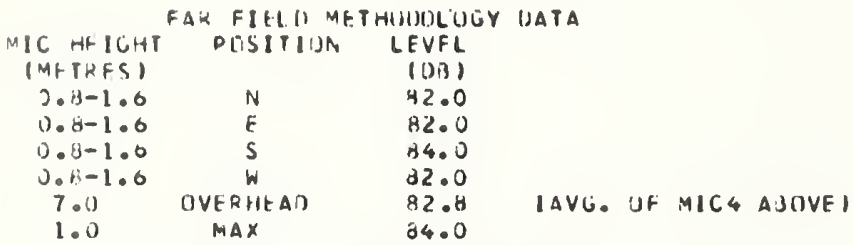

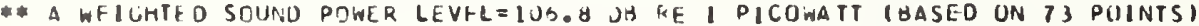

JUL IAN DAY 156 TEST NUMBER 3

NEAR FIELU DATA PRESENTATION

$$
\begin{gathered}
\text { MNURTH SIDE } \\
\text { 2M IM CENTER IM } \\
W
\end{gathered}
$$

MIC

HE IGHT

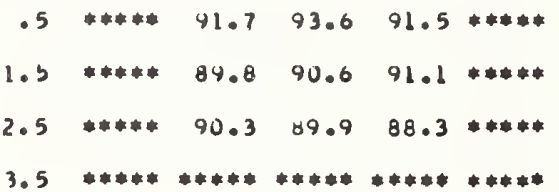

*TUP*

$\begin{array}{lllll}\text { MIC } & & & & \\ \text { HFICHY } & 0.5 & 1.5 & 2.5 & 3.5\end{array}$

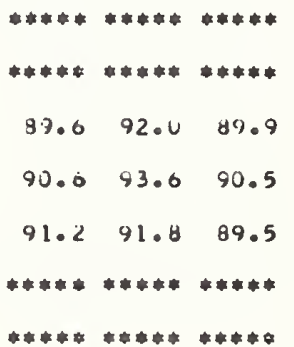

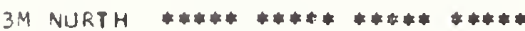

2M NUKTH $89.8 \quad 8 \% .2 \quad 68.9$ *****

lin Nisth $92.290 .0 \quad 80.2 * * * *$

CEINTERINE $92.2 \quad 90.8 \quad 69.4 \quad * * * *$

IM SOUTH

$92.9 \quad 41.0 \quad 90.4 \quad * * * *$

2M SUUTH

$89.890 .0 \quad 80.6 * * * *$

3M SOUTH

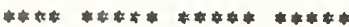

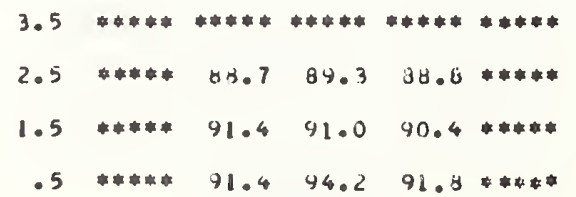

SUUTH SIOL $\begin{array}{lllll}3.5 & 2.5 & 1.5 & 0.5\end{array}$

EAST SIDE*

$\begin{array}{rrrr}3.5 & 2.5 & 1.5 & 0.5 \\ * * * * * * * * * * * * * * * * * * \\ * * * * * & 88.4 & 87.0 & 90.1 \\ * * * * * & 68.6 & 89.9 & 90.6 \\ * * * * * & 90.0 & 91.1 & 91.5 \\ * * * * * & 89.0 & 91.4 & 91.4 \\ * * * * * & 87.8 & 04.5 & 89.8 \\ * * * * * * * * * * * * * * * * * * *\end{array}$


Table 3.4

A-WEIGHTED SOUND PRESSURE LEVEL DATA

Test Number 4 Compressor Output: 150 cfm

Engine/Compressor Type: Rotary screw, Diesel, Standard

Compressor Size: $1.71 \times 1.24 \times 1.40 \mathrm{~m}$

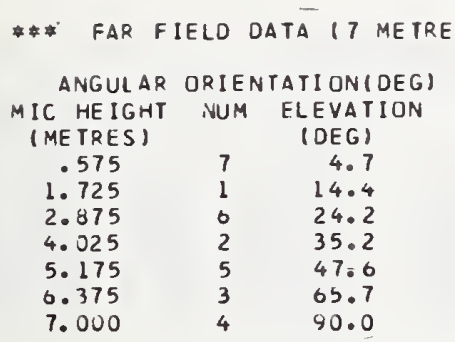

RADIUS )

FAR FIELD METHODOLOGY DATA

MIC HEIGHT POSITION LEVEL

(METRES) (D४)

$0.8-1.6 \quad N \quad 89.5$

$0.8-1.6 \quad E \quad 84.0$

$0.8-1.6 \quad S \quad 90.5$

$0.8-1.6$ W 88.5

7.0 OVERHEAD 86.9

1.0 MAX 92.0

(AVG. OF MIC4 ABOVE)

* a WeIghted suUnd pUWer level=113.1 d8 Re 1 PICOWATt (BASED ON 73 POINTS)

NEAR FIELD DATA PRESENTATION
TEST NUMBER 4
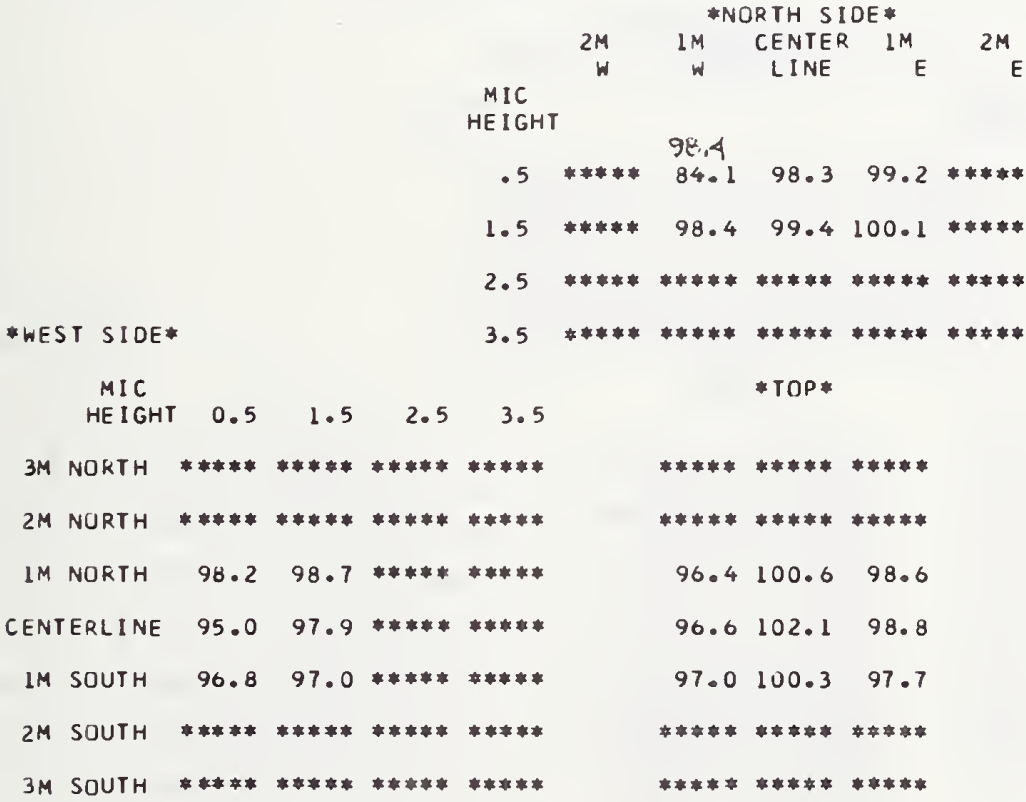

HE IGHT

$.5 * * * * * \begin{aligned} & 98.4 \\ & 84.1\end{aligned} \quad 98.3 \quad 99.2 * * * * *$

$.5 * * * * * 98.499 .4100 .1 * * * * *$

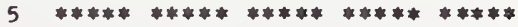

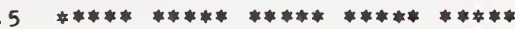

EAST SIDE*

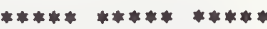

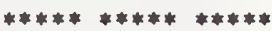

$96.4 \quad 100.6 \quad 98.6$

$96.6 \quad 102.1 \quad 98.8$

$97.0 \quad 100.3 \quad 97.7$

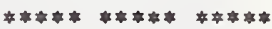

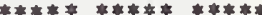
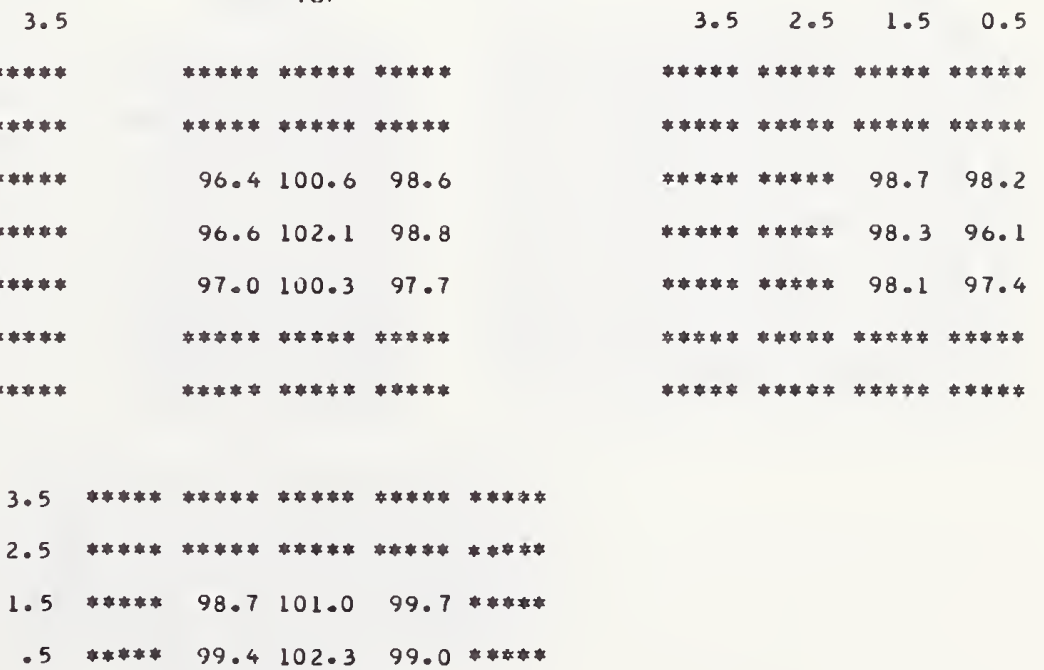

SOUTH SIDE* 
Table 3.5

A-WEIGHTED GOUND PRESSURE LEVEL DATA

Teet Number 5 Compressor Output: $200 \mathrm{cfm}$

Engine/Compressor Type: Reciprocating, Diesel, Standard

Compressor S1ze: $1.83 \times .98 \times 1.50$ m
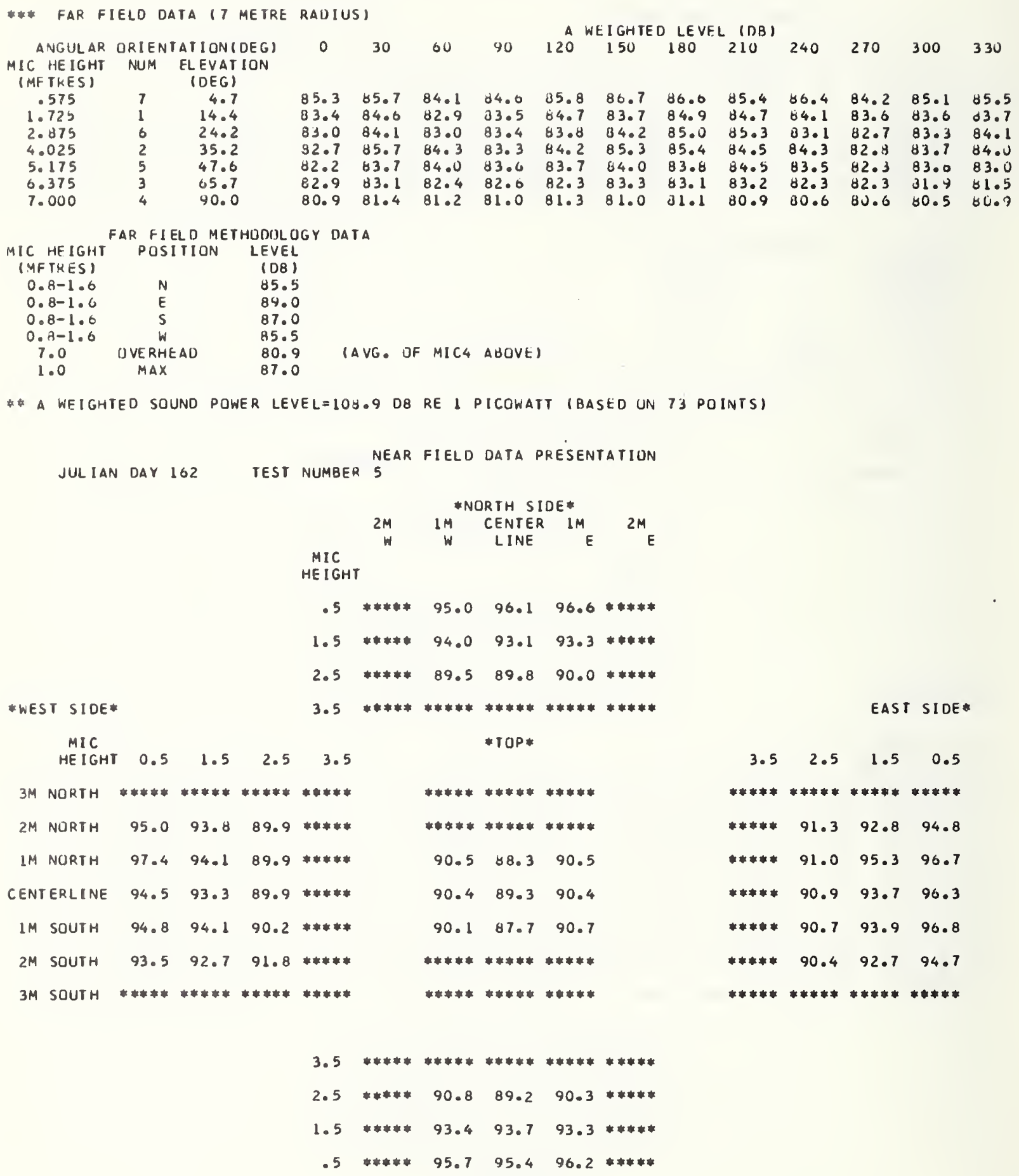
Table 3.6

A-WEIGHTED SOUND PRESSURE LEVEL DATA

Test Number 6 Compressor Output: $100 \mathrm{cfm}$

Engine/Compressor Type: Reciprocating, Diesel, Standard

Compressor S1ze: $1.52 \times .67 \times 1.42 \mathrm{~m}$

\section{* * far fielo data 17 metre radius)}

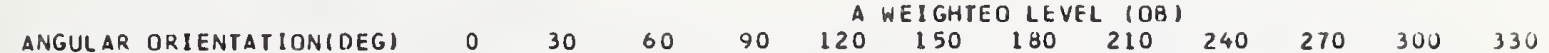
MIC HEIGHT NUM ELEVATIUN (ME TRES)

(OEG)

$\begin{array}{lll}.575 & 7 & 4.7\end{array}$

$1.725 \quad 1 \quad 14.4$

$2.875 \quad 6 \quad 24.2$

$4.025 \quad 2 \quad 35.2$

$5.175 \quad 5 \quad 47.6$

$6.375 \quad 3 \quad 65.7$

$\begin{array}{llllllllllll}84.0 & 84.9 & 85.1 & 84.6 & 84.1 & 85.3 & 86.9 & 86.3 & 88.3 & 85.4 & 87.1 & 84.7 \\ 82.3 & 83.0 & 83.9 & 82.1 & 82.7 & 84.2 & 83.5 & 83.8 & 84.6 & 83.6 & 86.5 & 83.3 \\ 81.5 & 82.6 & 81.4 & 81.6 & 83.2 & 84.6 & 85.3 & 85.2 & 86.7 & 83.3 & 83.7 & 83.0 \\ 83.3 & 82.8 & 82.7 & 83.0 & 83.9 & 85.0 & 85.1 & 84.9 & 84.8 & 83.4 & 82.5 & 82.9 \\ 82.2 & 83.2 & 83.8 & 84.1 & 84.1 & 83.9 & 84.1 & 84.2 & 85.2 & 84.8 & 83.2 & 83.2 \\ 83.9 & 83.9 & 85.4 & 83.1 & 82.8 & 83.8 & 84.6 & 84.0 & 83.5 & 83.0 & 82.9 & 82.9 \\ 82.2 & 81.7 & 83.3 & 82.3 & 82.7 & 82.1 & 82.0 & 81.3 & 81.9 & 81.9 & 82.7 & 82.3\end{array}$

FAR FIELO METHOOOLOGY OATA

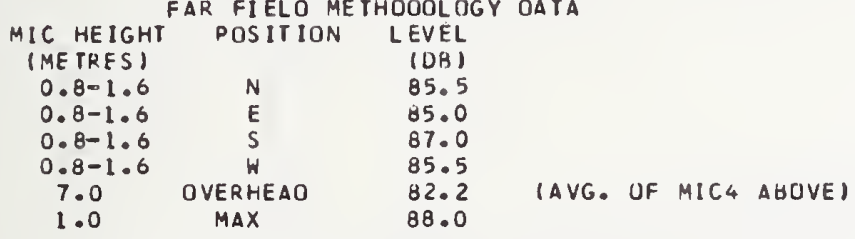

* a WeIghteo sound poner leVel=109.0 D8 Re I PICOWATt (BASE0 ON 73 puINTS)

JUL IAN DAY 163 TEST NUMBER 6

NEAR FIELD DATA PRESENTATION

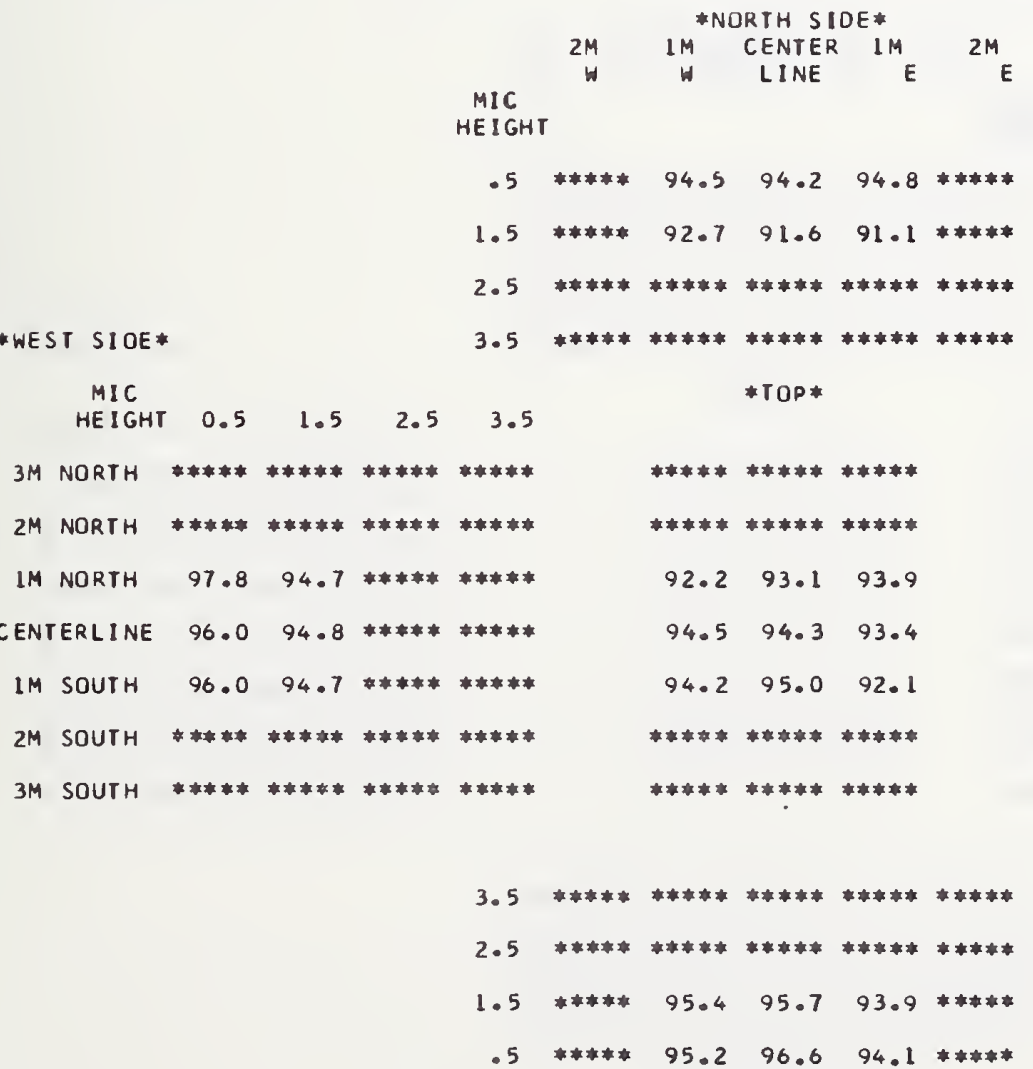

SOUTH SIDE* 
Table 3.7

A-WEIOHTHD BOUND PRESSURE LEVEL DATA

Test Number $7 \quad$ Compressor Output: 160 efm

Engine/Compressor Type: Rotary vane, Gao, Qu1eted

Compressor S1ze: $2.10 \times 1.28 \times 1.78 m$

* * far field data (t metre radius)

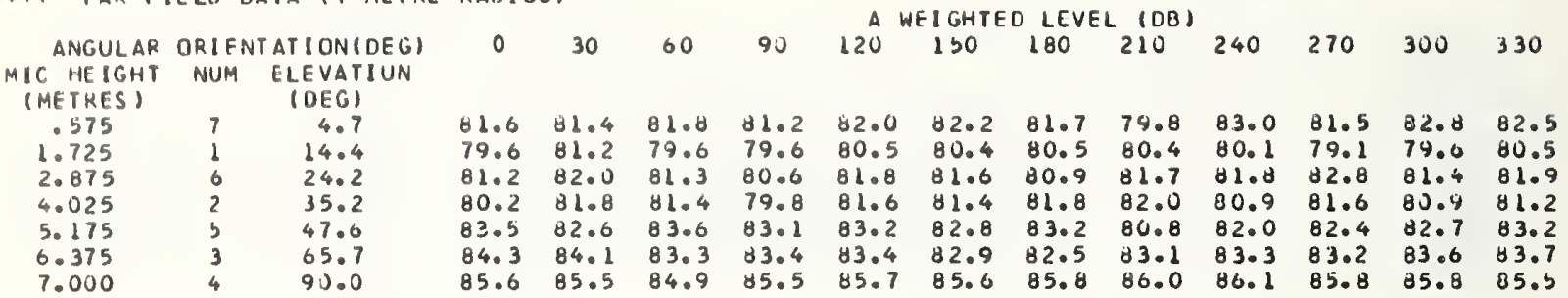

FAR FIELD METHODOLOGY UATA

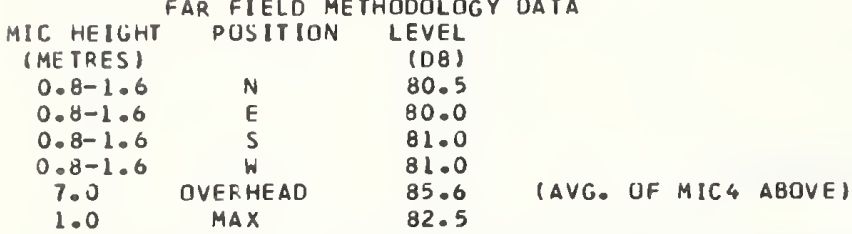

* a heighteo sound power level $=106.9$ db re l picunatt (baseo on 73 points)

JUL IAN DAY 164 TEST NUMBER 7

NEAR FIELO DATA PRESENTATION

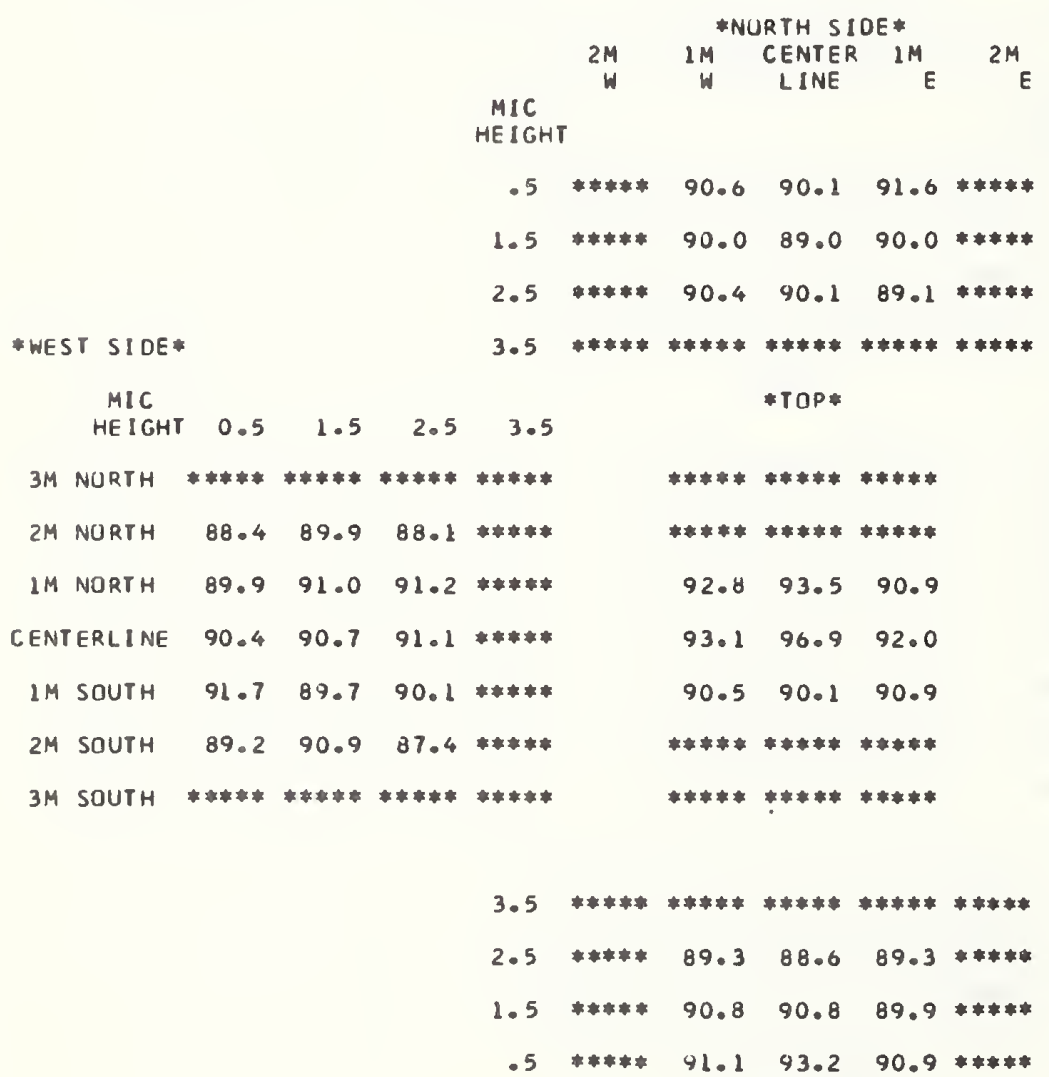

SOUTH SIDE*

EAST SIDE*

$\begin{array}{rrrr}3.5 & 2.5 & 1.5 & 0.5 \\ * * * * * * * * * * * * * * * & * * * * * \\ * * * * * & 89.1 & 90.3 & 87.4 \\ * * * * * & 89.9 & 90.7 & 89.8 \\ * * * * * & 90.2 & 89.9 & 89.3 \\ * * * * * & 90.1 & 90.8 & 91.0 \\ * * * * * & 88.1 & 89.1 & 89.3 \\ * * * * * * * * * * * * * * * * * * *\end{array}$


Table 3.8

A-WEIGHTED SOUND PRESSURE LEVEL DATA

Test Number $8 \quad$ Compressor Output: $125 \mathrm{cfm}$

Engine/Compressor Type: Rotary vane, Gas, Quleted

Compressor Size: $1.78 \times 1.30 \times 1.85$ m

* * far fielo data (t metre raoius)

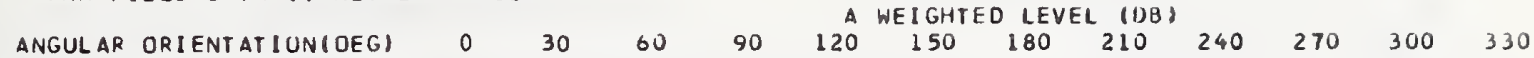
MIC HEIGHT NUM ELEVATIUN

(METRES)

$\begin{array}{rrr}.575 & 7 & 4.7\end{array}$

$1.725 \quad 1 \quad 14.4$

$2.875 \quad 6 \quad 24.2$

$35 \cdot 2$

4. 025

5.175

6.375

7.000

$$
47.6
$$

65.7

$\begin{array}{lllllllllllll}74.5 & 75.3 & 76.5 & 76.6 & 79.3 & 78.8 & 77.9 & 77.1 & 78.1 & 77.7 & 78.2 & 76.8\end{array}$

$\begin{array}{llllllllllll}75.1 & 75.6 & 76.3 & 75.4 & 76.1 & 77.5 & 76.8 & 76.2 & 76.4 & 76.6 & 76.6 & 76.1\end{array}$

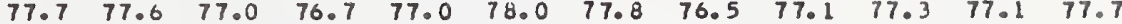

$\begin{array}{llllllllllll}75.4 & 76.6 & 76.9 & 76.9 & 77.9 & 79.0 & 77.6 & 77.4 & 77.9 & 78.0 & 77.0 & 77.1\end{array}$

$\begin{array}{llllllllllll}79.8 & 79.6 & 79.2 & 78.1 & 78.0 & 79.2 & 79.1 & 79.3 & 79.0 & 79.6 & 80.1 & 79.6\end{array}$

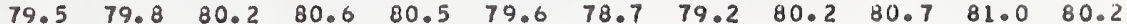

90.0

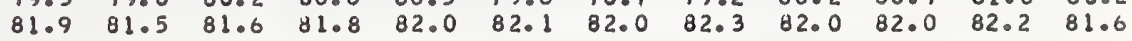

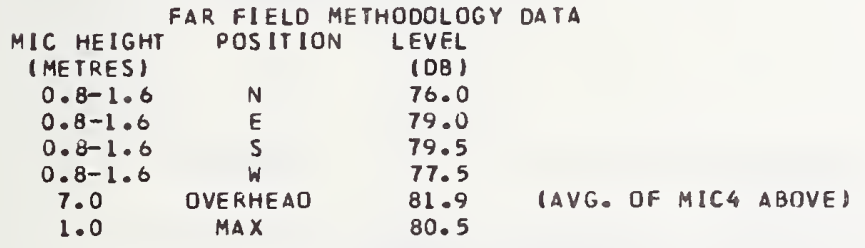

* A WEIGHTEO SOUND POWER LEVEL $=103.1$ DB RE 1 PICOWATT (8ASED ON 73 POINTS) JUL IAN OAY 165 TEST NUMBER 8

NEAR FIELO OATA PRESENTATION

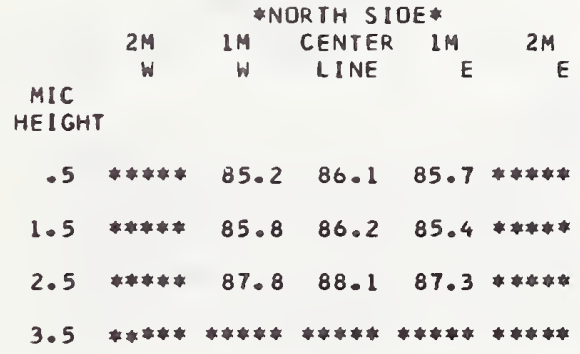

EAST SIDE*

*WEST SIDE*

*TOP*

$\begin{array}{lllll}\text { MIC } & & & & \\ \text { HEIGHT } & 0.5 & 1.5 & 2.5 & 3.5\end{array}$

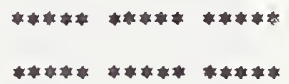

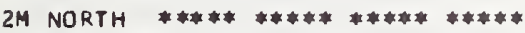

IM NORTH $86.5 \quad 86.2 \quad 87.9 \quad \%$ \#\#

$89.8 \quad 90.8 \quad 88.2$

CENTERLINE $86.0 \quad 86.5 \quad 88.2 \quad * * * * *$

$89.4 \quad 91.8 \quad 89.4$

$85.7 \quad 86.9 \quad 86.3$

$3.5 \quad 2.5 \quad 1.5 \quad 0.5$

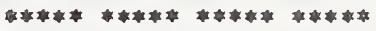

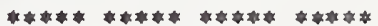

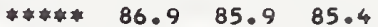

***** $87.1 \quad 85.6 \quad 85.2$

1M SOUTH $86.3 \quad 85.9 \quad 86.1$ *\#*

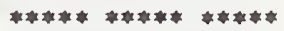

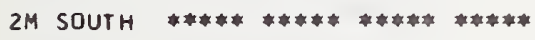

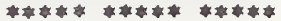

$* * \quad 85.2 \quad 86.0 \quad 86.5$

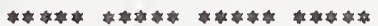

3M SOUTH

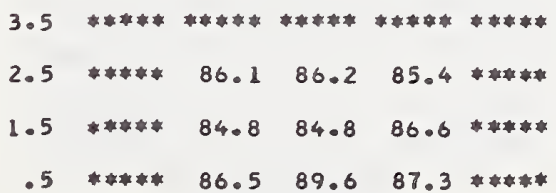

SOUTH SIDE* 
Table 3.9

A-WEIGHTED SOUND PRESSURE LEVEL DATA

Test Number 9 Compressor Output: $365 \mathrm{cfm}$

Engine/Compressor Type: Rotary vane, D1ebel, Quieted

Compressor S1ze: $3.66 \times 1.82 \times 2.14 \mathrm{~m}$

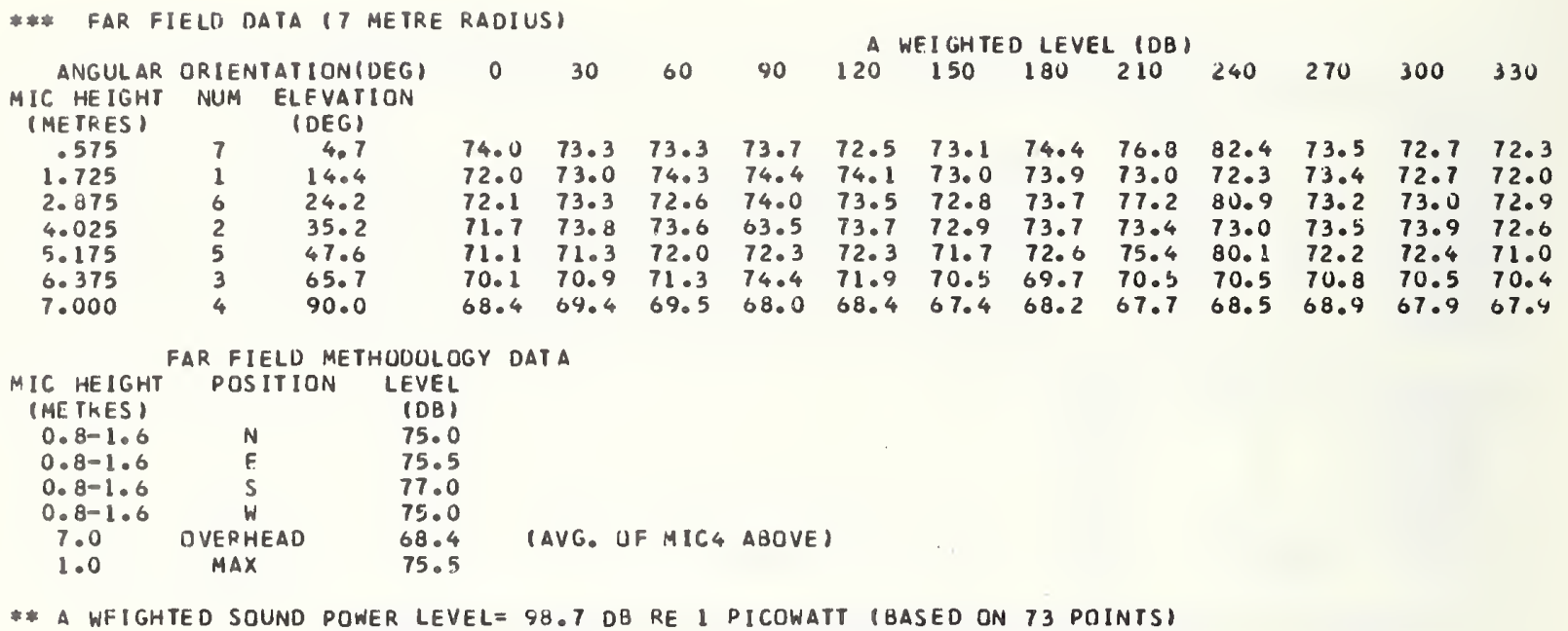

JUL IAN DAY 168 TEST NUMBER 9

NEAR FIELD DATA PRESENTATION

\begin{tabular}{|c|c|c|c|c|c|c|c|c|c|c|}
\hline & & & & & & & *NO & DRTH SI & DE & \\
\hline & & & & & & $2 M$ & $1 M$ & CENTER & $1 M$ & $2 M$ \\
\hline & & & & & & $W$ & $H$ & LINE & $E$ & $E$ \\
\hline & & & & & $\begin{array}{l}\text { MIC } \\
\text { HE IGHT }\end{array}$ & & & & & \\
\hline & & & & & $\cdot 5$ & $* 4 * 4 *$ & $81 \cdot 5$ & 41.6 & 82.1 & 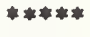 \\
\hline & & & & & 1.5 & $* * * *$ & $78 \cdot 2$ & 78.4 & 78.4 & ***3* \\
\hline & & & & & $2 \cdot 5$ & $* * * * *$ & $75 \cdot 8$ & 76.7 & 76.1 & 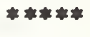 \\
\hline *WES & ST SIDE* & & & & $3 \cdot 5$ & $* * * * *$ & $* * * * *$ & 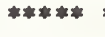 & $* * * * *$ & $* * * * 4$ \\
\hline & $\begin{array}{c}\text { MIC } \\
\text { HE I GHT }\end{array}$ & 0.5 & $1 \cdot 5$ & $2 \cdot 5$ & 3.5 & & & *TOP* & & \\
\hline $3 M$ & NORTH & *⿻三丨 & ****** & 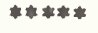 & 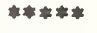 & & 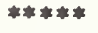 & 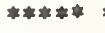 & 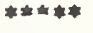 & \\
\hline $2 M$ & NORT H & $81 \cdot 8$ & $82 \cdot 1$ & 78.5 & 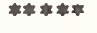 & & 74.8 & 75.9 & 75.7 & \\
\hline $1 M$ & NORTH & $82 \cdot 2$ & $81 \cdot 7$ & 80.2 & $\neq * * *$ & & $76 \cdot 6$ & 77.0 & 77.7 & \\
\hline CENT & ERLINE & 82.2 & 80.1 & 78.6 & 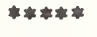 & & 79.1 & 80.6 & $78 \cdot 8$ & \\
\hline $1 M$ & SOUTH & $81 \cdot 6$ & 79.9 & $78 \cdot 2$ & 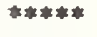 & & $79 \cdot 5$ & 81.8 & 79.6 & \\
\hline $2 M$ & SOUTH & 80.1 & $79 \cdot 3$ & 78.0 & ***** & & 76.3 & 76.9 & 76.1 & \\
\hline $3 M$ & SOUTH & 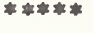 & 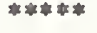 & *⿻三丨 & * & & 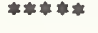 & 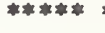 & $* 4 * 4 *$ & \\
\hline & & & & & $3 \cdot 5$ & 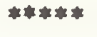 & $* * * *$ & 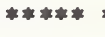 & $* * * * *$ & 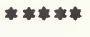 \\
\hline & & & & & $2 \cdot 5$ & 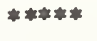 & $77 \cdot 8$ & 79.3 & 77.5 & 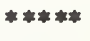 \\
\hline & & & & & $1 \cdot 5$ & 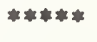 & $78 \cdot 3$ & $79 \cdot 2$ & 78.8 & 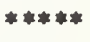 \\
\hline & & & & & .5 & 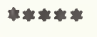 & 80.9 & 83.6 & $81 \cdot 4$ & 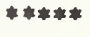 \\
\hline
\end{tabular}

SOUTH SIDE* 
Table 3.10

A-WEIGHTED SOUND PRESSURE LEVEL DATA

Test Number $10 \quad$ Compressor Output: $900 \mathrm{cfm}$

Engine/Compressor Type: Rotary vane, Diesel, Quleted

Compressor Size: $4.29 \times 2.19 \times 2.51 \mathrm{~m}$

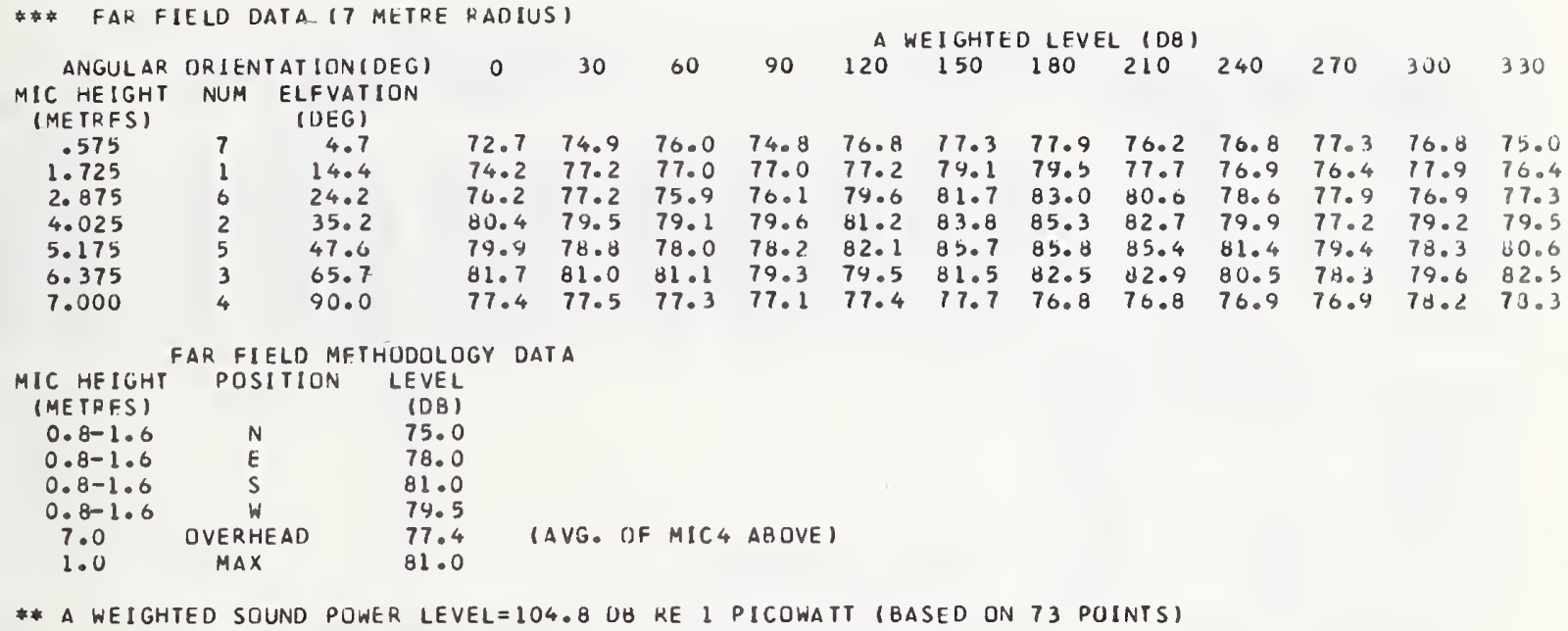

* a WeIghted SOUND POWER LEVEL=104.8 UB RE I PICOWATt (BASED ON 73 POINTS)

JUL IAN DAY 169 TEST NUMBER 10

NEAR FIELD DATA PRESENTATION
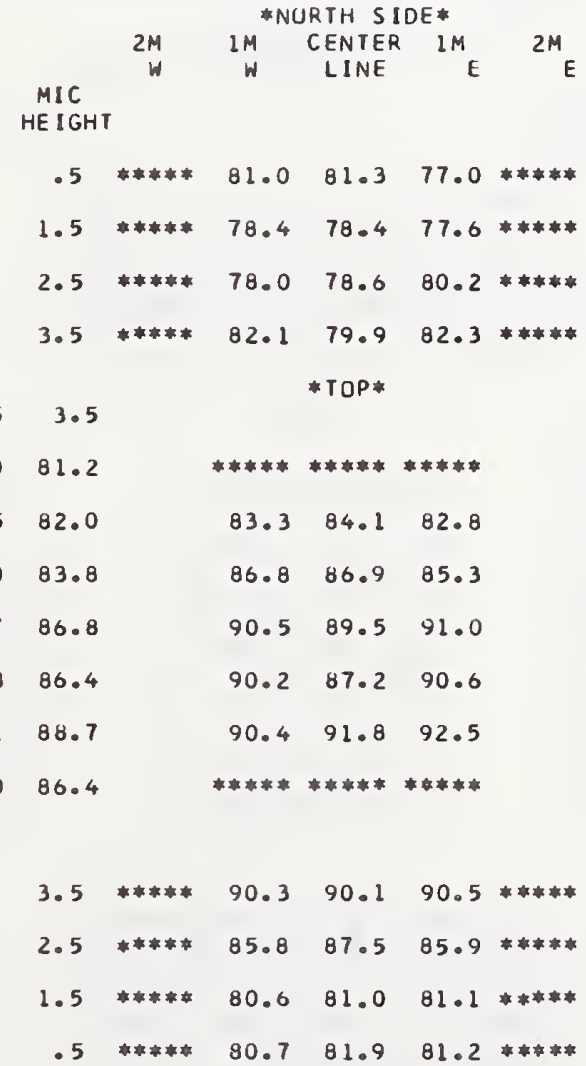

*WEST SIDE*

MIC

$\begin{array}{lllll}\text { HEIGHT } & 0.5 & 1.5 & 2.5 & 3.5\end{array}$

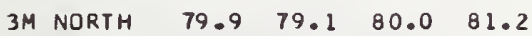

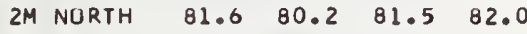

IM NORTH

$83.1 \quad 82.5 \quad 83.0 \quad 83.8$

CENTERL INE

IM SOUTH

2M SOUTH

3M SOUTH $\begin{array}{llll}82.7 & 81.9 & 84.7 & 86.8\end{array}$

$82.3 \quad 82.0 \quad 86.8 \quad 86.4$

$\begin{array}{llll}82.2 & 80.8 & 86.1 & 86.7\end{array}$

$79.6 \quad 81.4 \quad 85.0 \quad 86.4$ 
Table 3.11

A-WEIGHTED SOUND PRESSURE LEVEL DATA

Test Number 11 Compressor Output: $100 \mathrm{cfm}$

Engine/Compressor Type: Rotary acrew, Gas, Standard

Compressor Size: $1.78 \times 1.22 \times 1.37 \mathrm{~m}$

* * far fielo data (7 metre radius)

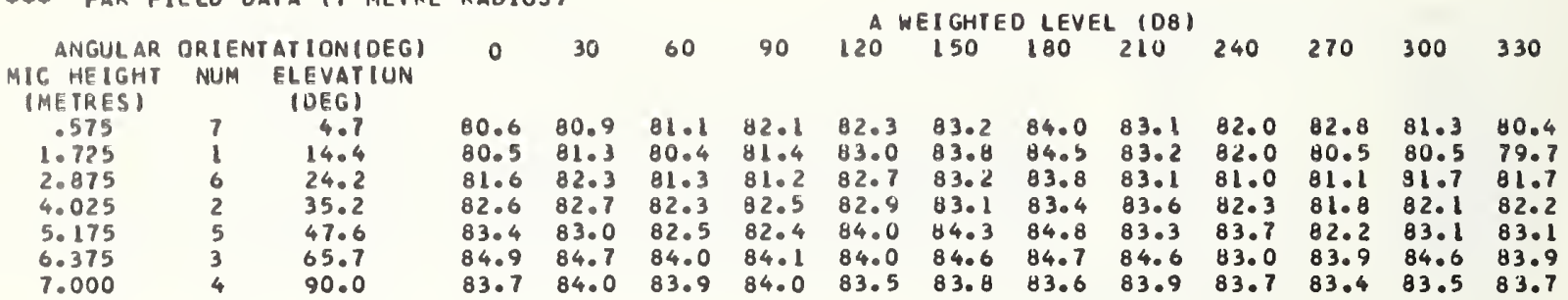

$\begin{array}{ccc}\text { FAR FIELD METHODOLOGY } \\ \text { MIC HEIGHT } & \text { POSITION } & \begin{array}{r}\text { LEVEL } \\ \text { ID I I }\end{array} \\ \text { (METRES) } & & 82.5 \\ 0.8-1.6 & \text { N } & 82.5 \\ 0.8-1.6 & \text { E } & 86.0 \\ 0.8-1.6 & \text { S } & 83.5 \\ 0.8-1.6 & \text { W } & 83.6 \\ 7.0 & \text { OVERHEAD } & 83.7 \\ 1.0 & \text { MAX } & 86.0\end{array}$

(AVG. OF MIC4 A8OVEI

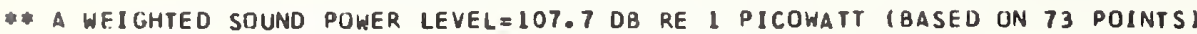

JULIAN DAY 176 TEST NUMBER 11

MIC

HE IGHT

$$
\begin{gathered}
\text { FNORTH SIDE* } \\
\text { WM IM CENTER IM } \\
\text { LINE }
\end{gathered}
$$

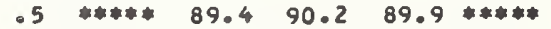

$1.5 \quad * * * * * \% 99.2 \quad 90.4 \quad 90.2 \quad * * * *$

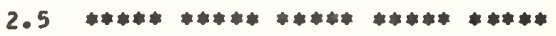

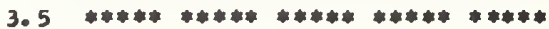

$\begin{array}{lllll}\text { HEIGHT } & 0.5 & 1.5 & 2.5 & 3.5\end{array}$

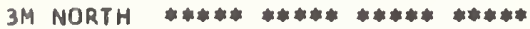

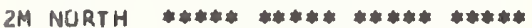

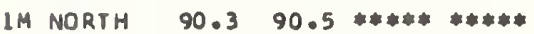

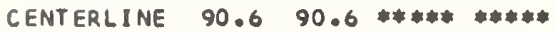

IM SOUTH $91.891 .6 * * * * * * * * *$

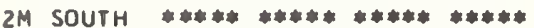

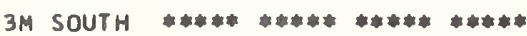

*TOP*

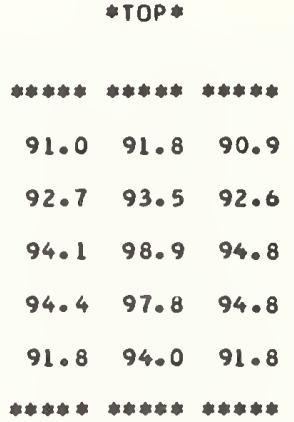

3.

2.

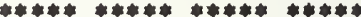

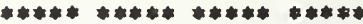

$* * * * * * * * * * \quad 90.4 \quad 90.4$

$* * * * * * * * * * 00.2 \quad 91.2$

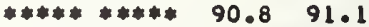

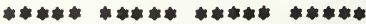

$* * * * * * * * * * * * * * * * * * * *$

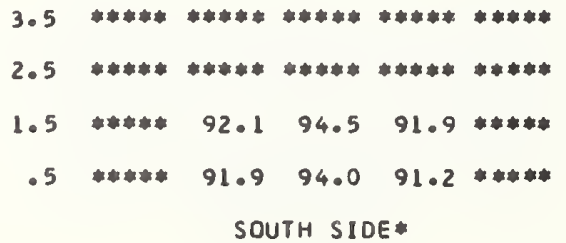


Table 3.12

A-WEIGHTED SOUND PRESSURE LEVEL DATA

Test Number 12 Compressor Output: $175 \mathrm{cfm}$

Engine/Compressor Type: Rotary screw, Gas, Quieted

Compressor Size: $2.70 \times 1.29 \times 1.43 \mathrm{~m}$

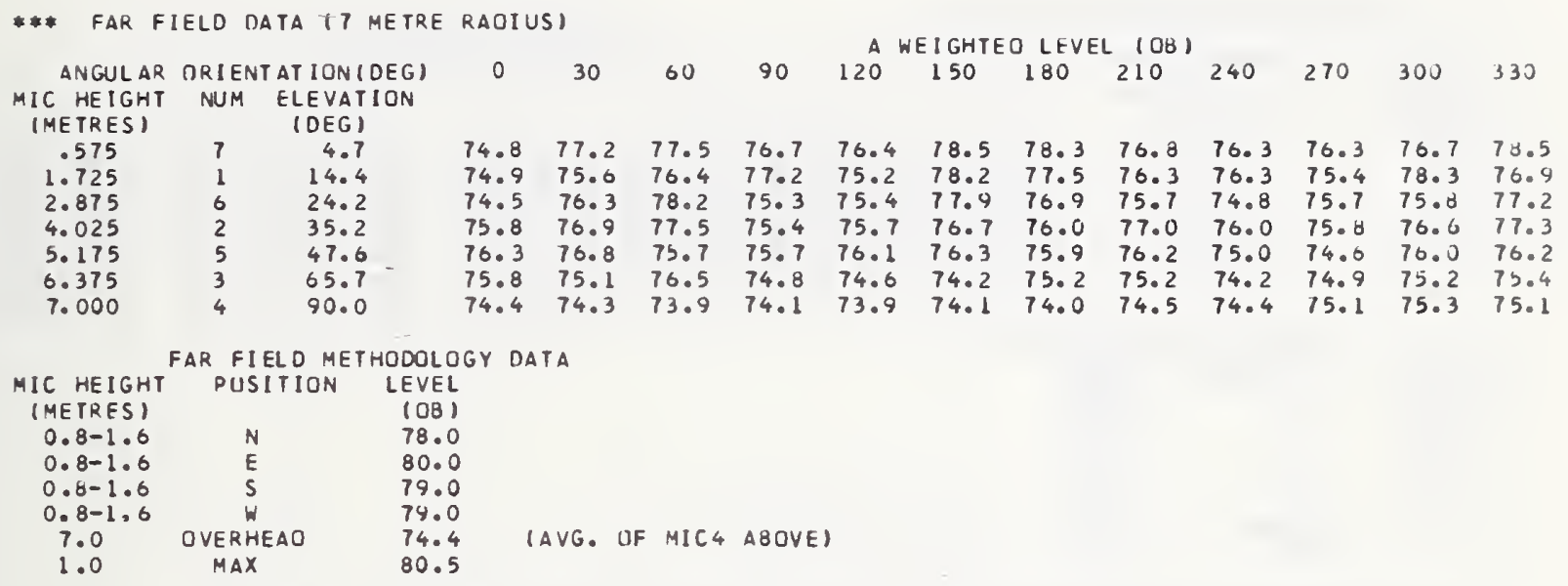

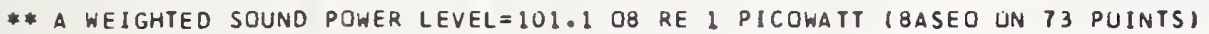

JUL IAN DAY 178 TEST NUMBER 12

NEAR FIELO OATA PRESENTATION

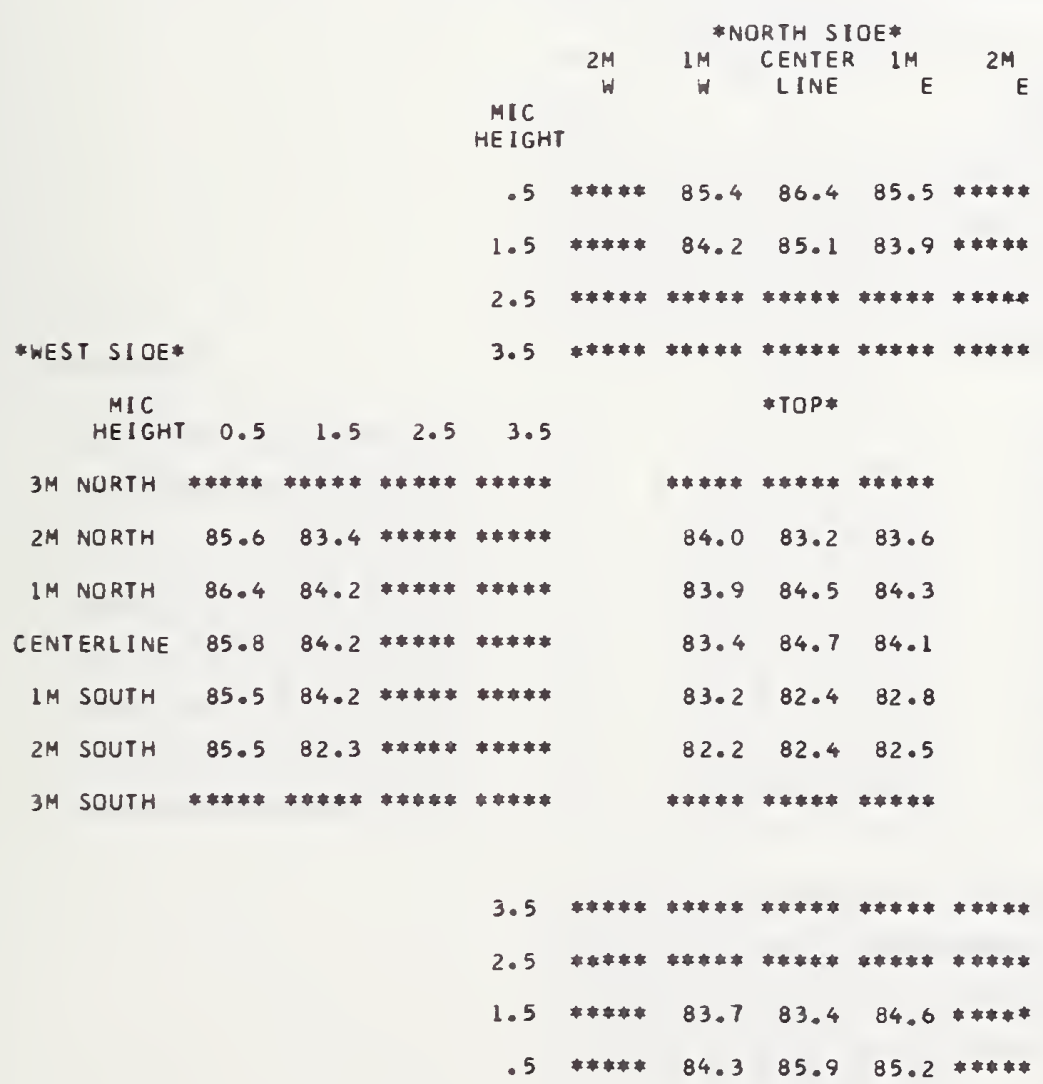

SOUTH SIDE* 
Table 3.13

A-WTIOHTED GOUND PRESEURE LEVEL DATA

Teat Nurber 13 Compressor Output: $175 \mathrm{cfm}$

EngIne/Compreator Type! Rotary acrew, Dleae1, QuLeted

Compreseor S12e: $2.70 \times 1.29 \times 1.43 \mathrm{~m}$

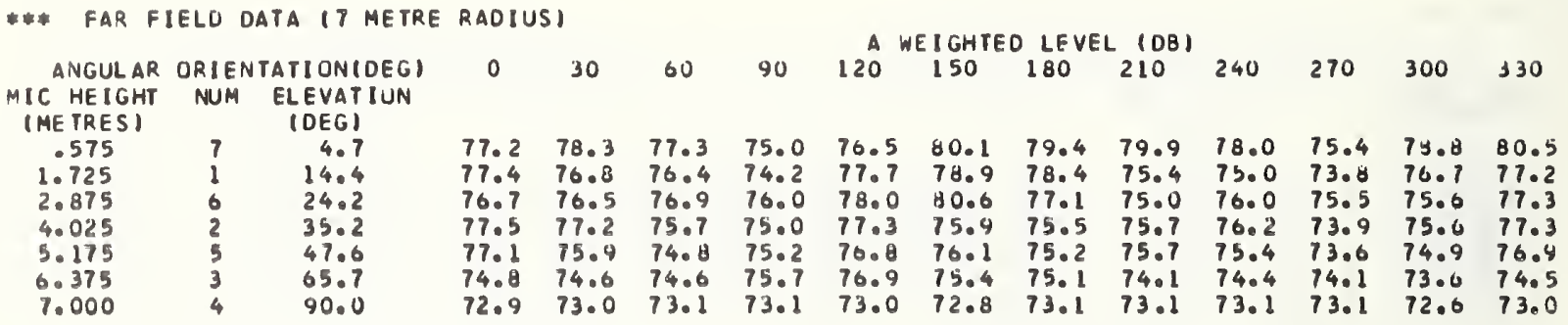

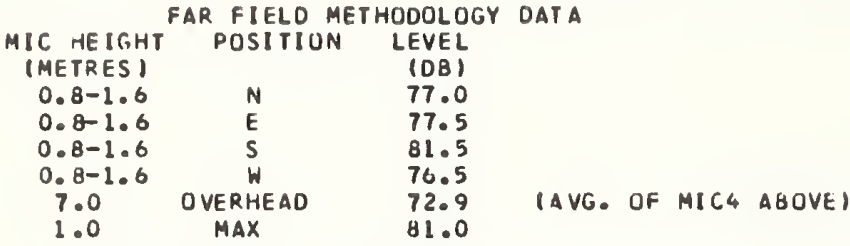

* a weighted sound power levela10l.4 dB Re l picowatt (Based on 73 puInts)

JULIAN DAY 183 TEST NUMBER 13

NEAR FIELO DATA PRESENTATION

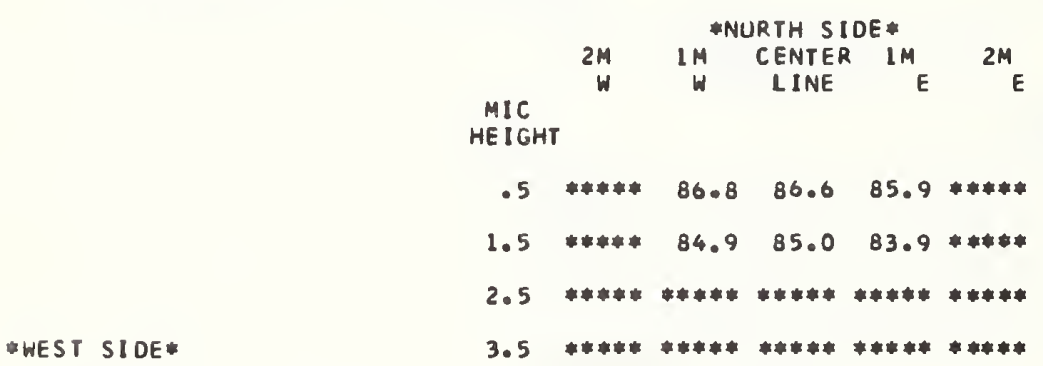

EAST SIDE*

$\begin{array}{lllll}\text { MIC } & & & & \\ \text { HEIGHT } & 0.5 & 1.5 & 2.5 & 3.5\end{array}$

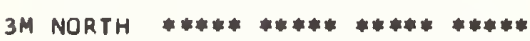

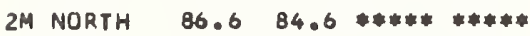

IM NORTH $87.983 .9 * * * * * * * *$

CENTERLINE $86.2 \quad 83.2 \quad * * * * * * * * * *$

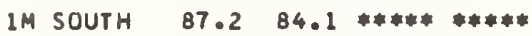

$2 M$ SOUTH $84.3 \quad 85.1 * * * * * * * * *$

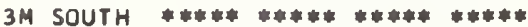

*TOP*

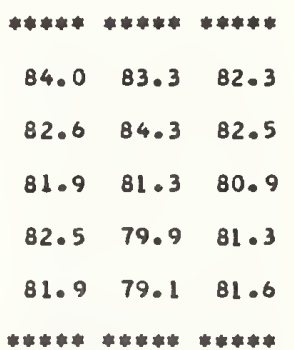

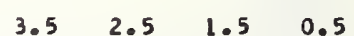

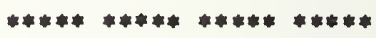

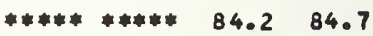

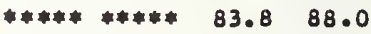

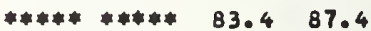

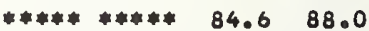

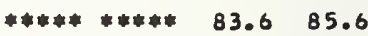

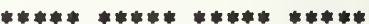

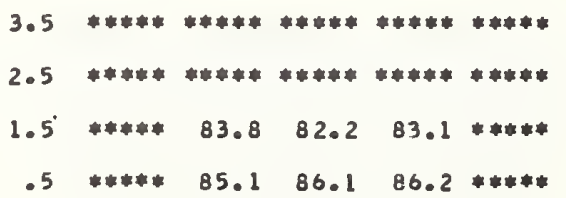

SUUTH SIDE* 
Table 3.14

A-WEIGHTED SOUND PRESSURE LEVEL DATA

Test Number 14 Compressor Output: $185 \mathrm{cfm}$

Engine/Compressor Type: Rotary screw, D1esel, Standard

Compressor Size: $2.70 \times 1.29 \times 1.43 \mathrm{~m}$

** far field data ( 7 metre radius)

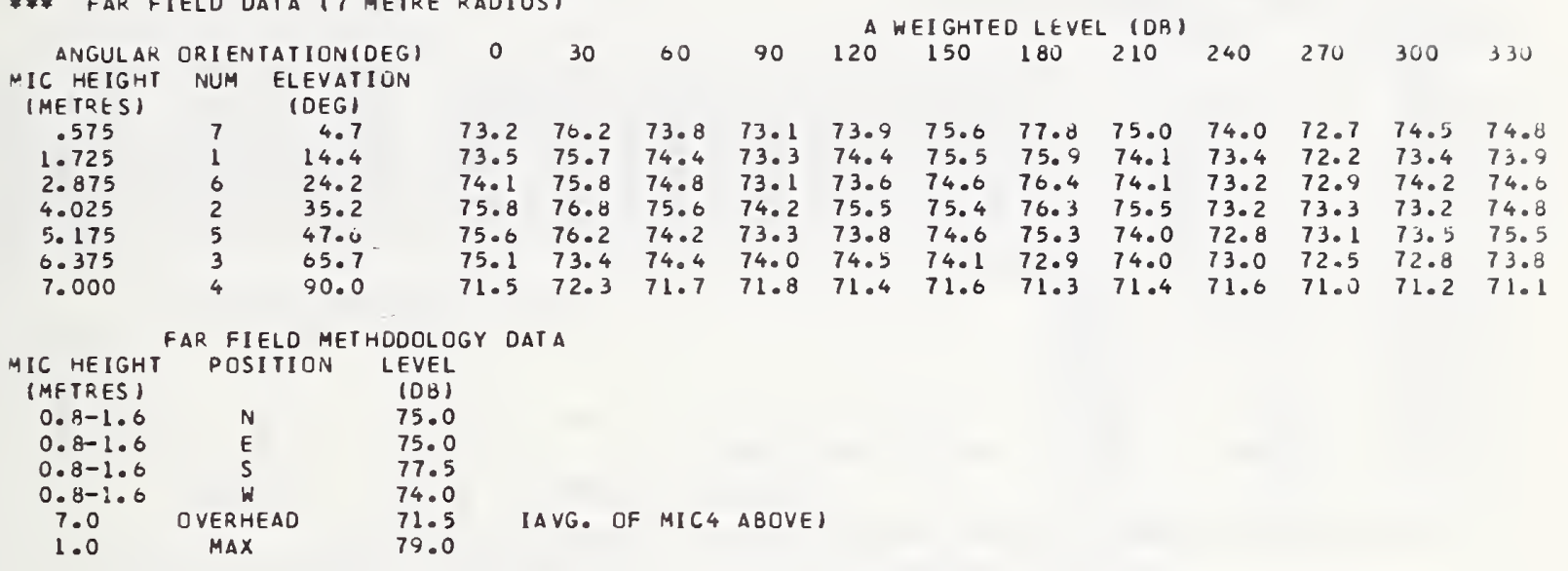

* a WEIGHTED SOUND POWER LEVEL $=99.3$ DB RE 1 PICUWATt (8ASED ON 73 POINTS)

JUL IAN DAY 184 TEST NUMBER 14

NEAR FIELD DATA PRESENTATIDN

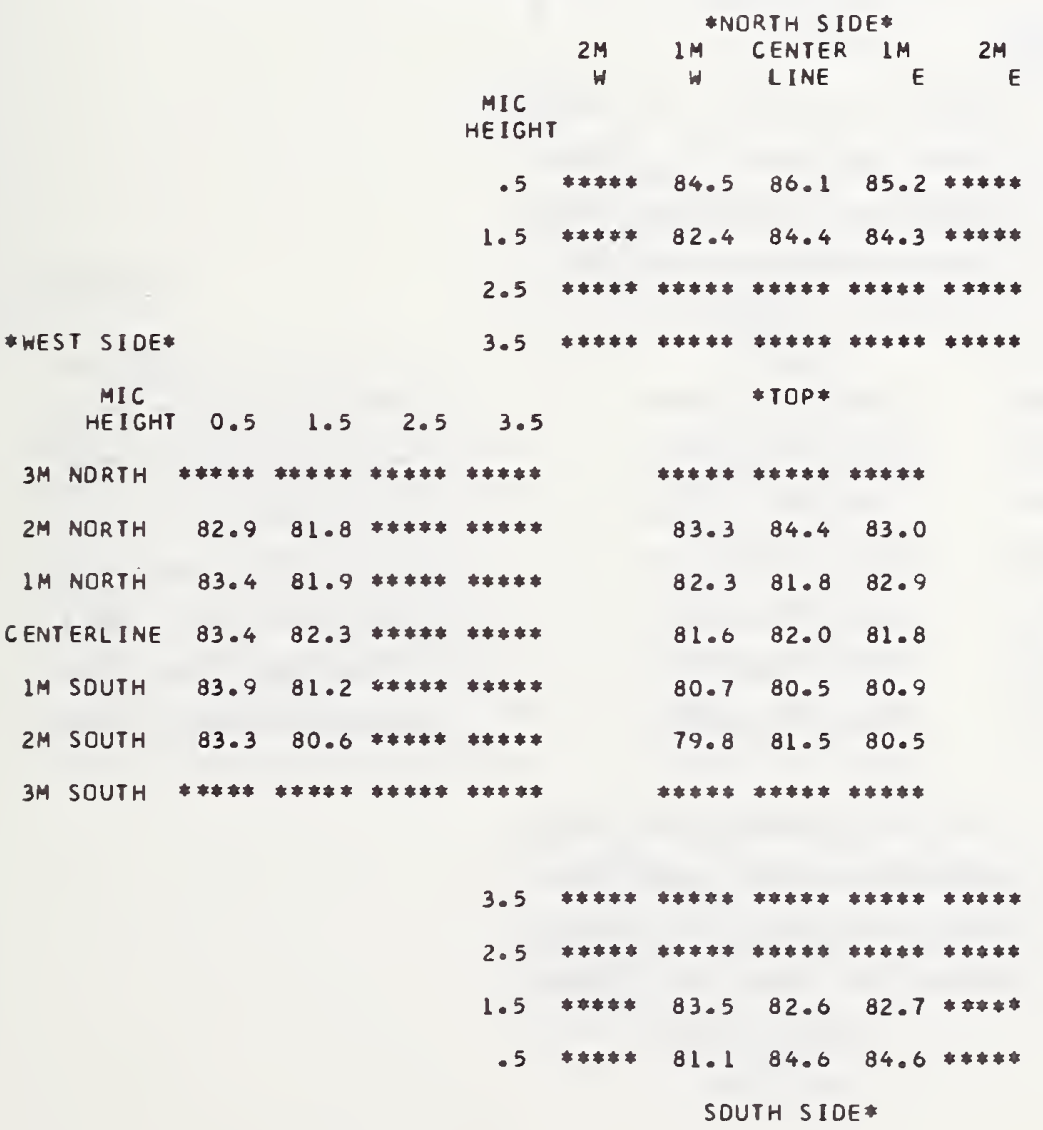


Table 3.15

A-WEIGHTED SOUND PRESSURE LEVEL DATA

Test Number 15 Compressor Output: $175 \mathrm{cfm}$

Engine/Compressor Type: Rotary screw, Gae, Stendard

Compressor Size: $1.99 \times 1.27 \times 1.45 \pi$

* * far field data 17 metre radius)

\begin{tabular}{|c|c|c|c|c|c|c|c|c|c|c|c|c|c|c|}
\hline & & & & & & & A & EI GHT & LEVE & $(0 B)$ & & & & \\
\hline $\begin{array}{c}\text { MIC HEIGHT } \\
\text { (MF TRES) }\end{array}$ & AUM & $\begin{array}{l}\text { ELEVAT IUN } \\
\text { (DEG) }\end{array}$ & & & & & & & & & & & & \\
\hline $\begin{array}{r}.575 \\
1.725 \\
2.875 \\
4.025 \\
5.175 \\
6.375 \\
7.000\end{array}$ & $\begin{array}{l}7 \\
1 \\
6 \\
2 \\
5 \\
3 \\
4\end{array}$ & $\begin{array}{l}4.7 \\
14.4 \\
24.2 \\
35.2 \\
47.6 \\
65.7 \\
90.0\end{array}$ & $\begin{array}{l}82.2 \\
81.1 \\
81.1 \\
83.6 \\
82.9 \\
84.4 \\
83.0\end{array}$ & $\begin{array}{l}82.0 \\
82.3 \\
32.1 \\
83.5 \\
83.6 \\
84.6 \\
82.9\end{array}$ & $\begin{array}{l}82.7 \\
82.9 \\
82.4 \\
83.3 \\
82.8 \\
84.1 \\
83.1\end{array}$ & $\begin{array}{l}82.5 \\
82.4 \\
82.6 \\
83.2 \\
83.6 \\
83.8 \\
83.1\end{array}$ & $\begin{array}{l}84.1 \\
83.6 \\
83.5 \\
83.3 \\
83.4 \\
84.2 \\
83.4\end{array}$ & $\begin{array}{l}86.0 \\
86.2 \\
84.6 \\
84.7 \\
84.9 \\
83.9 \\
82.8\end{array}$ & $\begin{array}{l}87.3 \\
86.8 \\
85.4 \\
85.9 \\
86.0 \\
83.5 \\
83.2\end{array}$ & $\begin{array}{l}86.3 \\
87.5 \\
34.2 \\
84.7 \\
85.1 \\
84.0 \\
82.6\end{array}$ & $\begin{array}{l}85.2 \\
63.1 \\
82.2 \\
82.8 \\
83.6 \\
84.6 \\
83.1\end{array}$ & $\begin{array}{l}83.3 \\
81.7 \\
81.8 \\
82.2 \\
83.8 \\
84.1 \\
83.2\end{array}$ & $\begin{array}{l}83.7 \\
81.6 \\
81.5 \\
32.4 \\
82.8 \\
84.5 \\
03.0\end{array}$ & $\begin{array}{l}82.4 \\
81.0 \\
81.8 \\
82.5 \\
83.1 \\
84.1 \\
82.0\end{array}$ \\
\hline $\begin{array}{l}\text { MIC HEIGHT } \\
\text { (ME TRES ) } \\
0.8-1.6 \\
0.8-1.6 \\
0.8-1.6 \\
0.8-1.6 \\
7.0 \\
1.0\end{array}$ & $\begin{array}{r}\text { FAR FI } \\
\text { POS } \\
\\
N \\
E \\
S \\
W \\
\text { OVERHE } \\
\text { MAX }\end{array}$ & 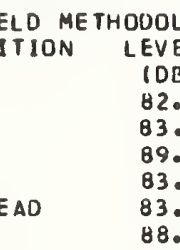 & & AVG. OF & $=\mathrm{MlC4}$ & ABOVE & & & & & & & & \\
\hline
\end{tabular}

* a heighteo sound power level=108.7 de re I picuwatt (Based on 73 points)

JUL IAN DAY 189 TEST NUMBER 15

\section{NEAR FIELO OATA PRESENTATION}

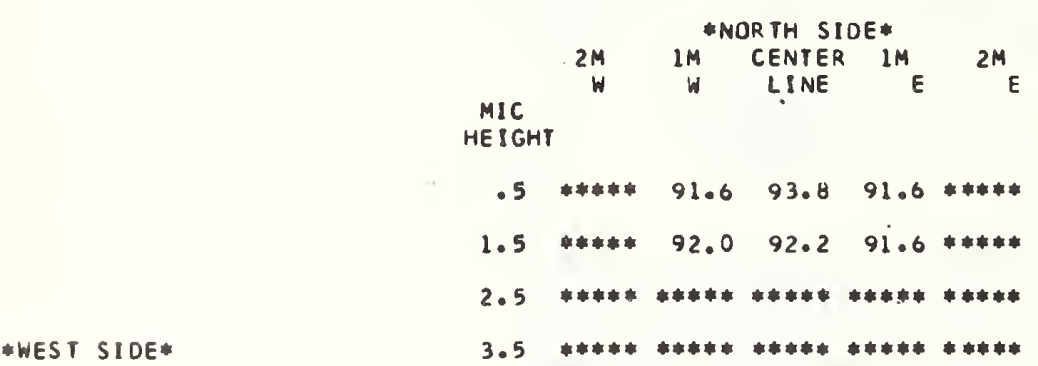

EAST SIDE*

MIC

HEIGHT $0.5 \quad 1.5 \quad 2.5 \quad 3.5$

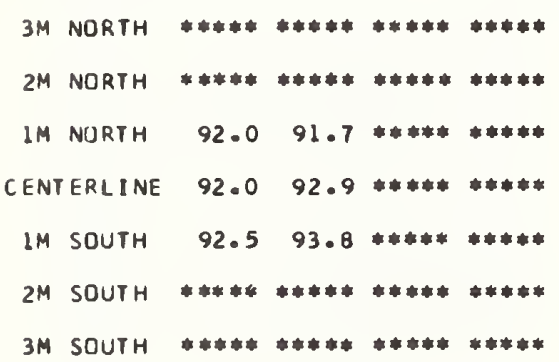

$*$ TOP*

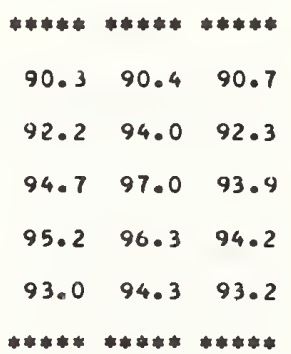
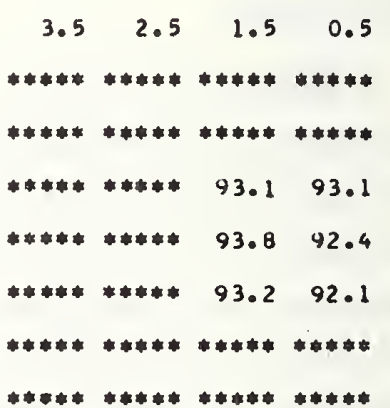

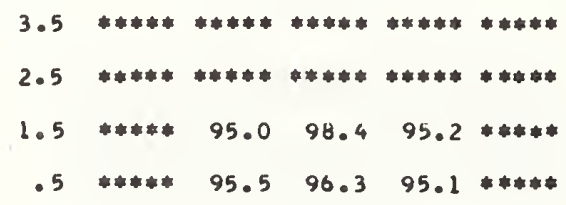

SUUTH SIDE* 
Table 3.16

A-WEIGHTED SOUND PRESSURE LEVEL DATA

Test Number 16 Compressor Output: $85 \mathrm{cfm}$

Englne/Compressor Type: Rotary screw, Gas, Standard

Compressor Size: $1.96 \times 1.10 \times 1.34 \mathrm{~m}$

* * far fIElo data $(7$ metre ral)iUs)

ANGULAR URIENTATIIIN(DEG) $\quad 0 \quad 30 \quad 60 \quad 90 \quad 120$ L $150 \quad 18 \mathrm{~J} 210 \quad 240 \quad 270 \quad 30 \mathrm{~J} 330$ MIC HE IGHT NUM ELEVATIUN

(ME TPFS)

$\begin{array}{lllllllllllllll}.575 & 7 & 4.7 & 76.5 & 76.4 & 76.0 & 70.1 & 77.5 & 78.6 & 79.4 & 70.2 & 76.1 & 75.3 & 75.1 & 70.5 \\ 1.725 & 1 & 14.4 & 74.1 & 74.5 & 74.4 & 73.8 & 75.8 & 78.1 & 79.1 & 73.2 & 75.6 & 74.2 & 73.1 & 74.3 \\ 2.375 & 6 & 24.2 & 74.0 & 74.0 & 74.6 & 73.7 & 76.3 & 78.0 & 77.9 & 77.6 & 15.4 & 73.7 & 73.8 & 74.7 \\ 4.075 & 2 & 35.2 & 74.0 & 72.0 & 74.6 & 74.2 & 70.2 & 78.4 & 79.3 & 78.6 & 77.2 & 75.4 & 75.1 & 75.3 \\ 5.175 & 5 & 47.6 & 75.9 & 76.2 & 75.9 & 75.7 & 76.9 & 78.4 & 78.9 & 78.5 & 77.2 & 75.6 & 75.5 & 75.7 \\ 6.375 & 3 & 05.7 & 75.3 & 74.6 & 74.3 & 74.5 & 75.8 & 76.6 & 77.2 & 76.7 & 75.9 & 75.3 & 74.6 & 74.1 \\ 7.000 & 4 & 90.0 & 74.1 & 74.1 & 74.2 & 74.0 & 73.6 & 74.5 & 74.0 & 74.5 & 74.3 & 74.3 & 74.4 & 74.2\end{array}$

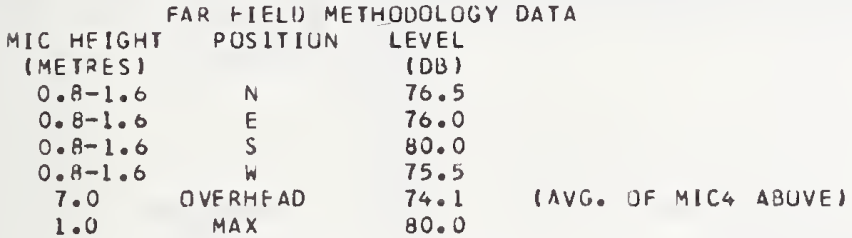

* a WFighted SOUND pOWER leVel=101.1 d8 fe I pICDWATt (BASED UN 73 PuINTS)

JUL IAN DAY 190

NEAR FIELD DATA PRESENTATION TEST NUMBER 16

HE I GHT

$$
\begin{array}{crcrr}
\multicolumn{4}{c}{\text { *NURTH SIDE* }} \\
2 M & \text { IM CENTER IM } & \text { 2M } \\
\text { W } & W & \text { LINE } & E & E
\end{array}
$$

$.5 * * * * 85.3 \quad 86.1 \quad 85.0 * * * 4$

$1.5 \quad * * * * \quad 83.5 \quad 83.7 \quad 83.1 \quad * * * *$

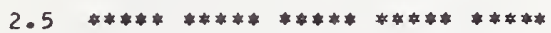

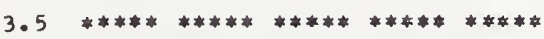

EAST SIDE*

*WEST SIDE*

*TOP*

$\begin{array}{lllll}\text { MIC } & & & & \\ \text { HEIGHT } & 0.5 & 1.5 & 2.5 & 3.5\end{array}$

$\begin{array}{lllll}\text { MIC } & & & & \\ \text { HE IGHT } & 0.5 & 1.5 & 2.5 & 3.5\end{array}$

trop*
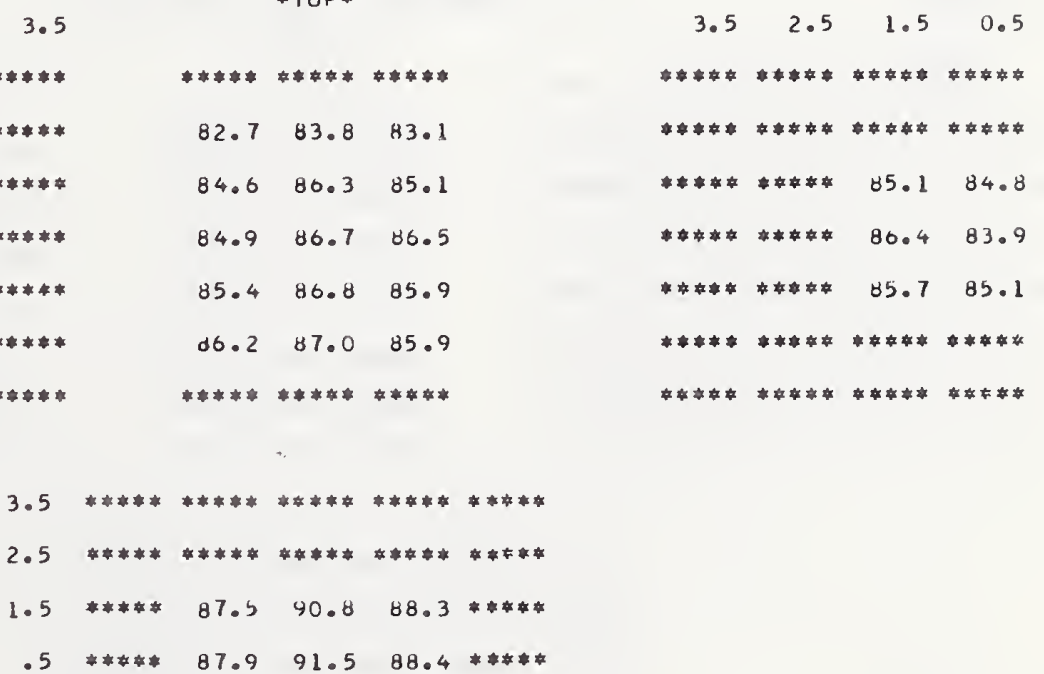

SUUTH SIDE* 
Table 3.17

A-WEIGHED SOUND PRESSURE LEVEL DATA

Teat Number 17 Compressor Output: $150 \mathrm{cfm}$

Engine/Compreseor Type: Rotazy screw, Gas, Standard

Compreseor S1ze: $1.93 \times 1.24 \times 1.36 \mathrm{~m}$

* * far field data 17 metre radiusi

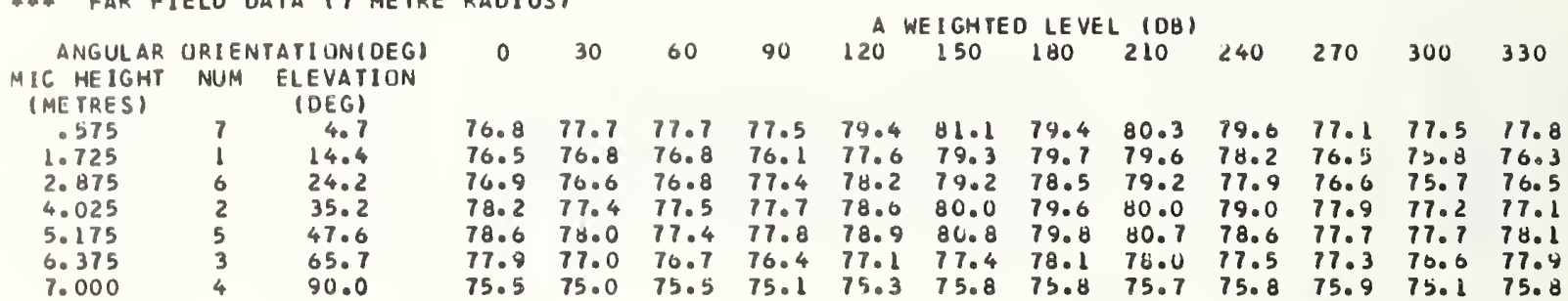

\begin{tabular}{ccc}
\multicolumn{3}{c}{ FAR FIELD METHOOOLOCY } \\
$\begin{array}{c}\text { MIC HEIGHT } \\
\text { ICOSITION }\end{array}$ LEVEL \\
(METRES) & & (DB) \\
$0.8-1.6$ & N & 77.5 \\
$0.8-1.6$ & E & 78.0 \\
$0.8-1.6$ & 5 & 80.0 \\
$0.8-1.6$ & W & 77.5 \\
7.0 & OVERHEAD & 75.5 \\
1.0 & MAX & 83.0
\end{tabular}

* a WEIGHTED SOUND POWER LEVEl=103.0 DB RE I PICOWATt (BASED UN 73 POINTS)

JULIAN DAY 191 TEST NUMBER 17

NEAK FIELD DATA PRESENTATION

\begin{tabular}{|c|c|c|c|c|c|c|c|c|c|}
\hline & & & & & & \#NO & RTH SI & DE* & \\
\hline & & & & & $2 M$ & $1 \mathrm{M}$ & CENTER & IM & $2 M$ \\
\hline & & & & & $w$ & $w$ & LINE & E & E \\
\hline & & & & MIC & & & & & \\
\hline & & & & HE IGHT & & & & & \\
\hline & & & & .5 & $* * * * *$ & 87.6 & 91.3 & 87.5 & $* * * * *$ \\
\hline & &. & & 1.5 & $* * * * *$ & 85.8 & 86.3 & 87.0 & 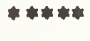 \\
\hline & & & 3 & 2.5 & $* * * * *$ & $* * * * *$ & 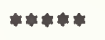 & $\neq * * * *$ & 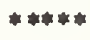 \\
\hline WEST SIDE* & & & & 3.5 & $* * * *$ & $* * * * *$ & $* * * * *$ & ****** & 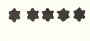 \\
\hline $\begin{array}{l}\text { MIC } \\
\text { HE I GHT }\end{array}$ & 0.5 & 1.5 & 2.5 & 3.5 & & & *TOP* & & \\
\hline 3M NORTH & 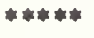 & 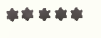 & 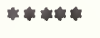 & $* * * * *$ & & 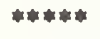 & $\neq * * * *$ & $* * * * *$ & \\
\hline 2M NURTH & 86.5 & 84.3 & 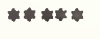 & $* * * * *$ & & 84.2 & 86.1 & 86.2 & \\
\hline IM NORTH & 87.6 & 86.3 & $* * * * *$ & ***** & & 87.4 & 89.7 & 87.3 & \\
\hline CENTERLINE & 87.9 & 86.9 & $* * * * *$ & 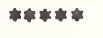 & & 89.3 & 90.6 & 89.1 & \\
\hline IM SOUTH & 89.4 & 87.6 & $* * * * *$ & $* * * * *$ & & 88.6 & 90.4 & 89.3 & \\
\hline $2 M$ SOUTH & 88.8 & 86.5 & 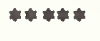 & 후후 & & 88.1 & 88.8 & 88.1 & \\
\hline 3M SOUTH & 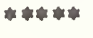 & $* * * * *$ & $* * * *$ & 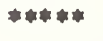 & & 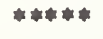 & 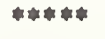 & $* * * *$ & \\
\hline & & & & 3.5 & ****** & 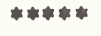 & 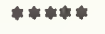 & $* * * * *$ & $* * * * *$ \\
\hline & & & & 2.5 & $* * * *$ & 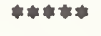 & 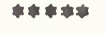 & 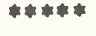 & $* * * * *$ \\
\hline & & & & 1.5 & $* * * * *$ & 90.2 & 90.1 & 90.3 & $* * * * *$ \\
\hline & & & & & 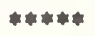 & 90.3 & 92.5 & 90.1 & * \\
\hline
\end{tabular}


Table 3.18

A-WEIGHTED SOUND PRESSURE LEVEL DATA:

Test Number 18 Broad Band Reference Sound Source

* far field data 17 hetre radius)

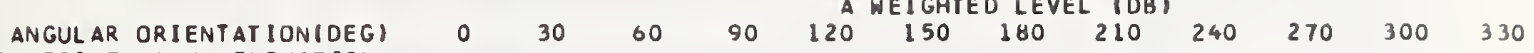
MIC HEIGHT NUM ELEVATION

(METRES) (DEG)

$\begin{array}{rrr}575 & 7 & 4.7 \\ 725 & 1 & 14.4\end{array}$

114.4

$2.875 \quad 6 \quad 24.2$

$4.025 \quad 2 \quad 35.2$

$5.175 \quad 5 \quad 47.6$

$\begin{array}{lll}6.375 & 3 & 65.7 \\ 7.000 & 4 & 90.0\end{array}$

$\begin{array}{lllllllllllll}71.4 & 71.0 & 71.0 & 72.0 & 72.1 & 71.7 & 71.3 & 72.1 & 71.9 & 72.0 & 72.1 & 71.3 \\ 70.8 & 71.5 & 70.5 & 70.4 & 70.2 & 70.7 & 71.2 & 71.0 & 71.3 & 71.5 & 71.4 & 71.2 \\ 70.2 & 69.9 & 70.3 & 70.3 & 70.5 & 70.0 & 69.6 & 69.7 & 69.6 & 70.1 & 70.4 & 70.1 \\ 69.8 & 69.9 & 70.1 & 70.3 & 70.6 & 71.1 & 70.7 & 70.6 & 70.7 & 70.3 & 70.7 & 70.6 \\ 69.6 & 69.6 & 69.8 & 70.1 & 70.0 & 70.1 & 68.7 & 69.7 & 69.3 & 69.2 & 69.0 & 69.7 \\ 69.5 & 70.0 & 69.8 & 69.6 & 69.3 & 69.4 & 69.7 & 69.8 & 70.7 & 70.2 & 70.2 & 70.1 \\ 69.4 & 69.4 & 69.2 & 69.5 & 69.2 & 69.3 & 69.5 & 69.9 & 69.8 & 69.8 & 69.6 & 69.6\end{array}$

FAR FIELD METHODOLOGY DATA

$\begin{array}{ccr}\text { MIC HEIGHT } & \text { PUSITION } & \text { LEVEL } \\ \text { (METRES ) } & & 708 \text { ) } \\ 0.8-1.6 & \text { N } & 72.0 \\ 0.8-1.6 & \text { E } & 73.0 \\ 0.8-1.6 & \text { S } & 73.0 \\ 0.8-1.6 & \text { W } & 73.0 \\ 7.0 & \text { OVERHEAD } & 69.4 \\ 1.0 & \text { MAX } & 72.5\end{array}$

(AVG. OF MIC4 ABOVE)

* a heighted sound power level=95.3 DB Re l pICOWATt (8ASED ON 73 poINTS) JUL IAN DAY 141 NEAR FIELD DATA PRESENTATION

MIC

\begin{tabular}{ccccc}
\multicolumn{4}{c}{ *NORTH SIDE* } \\
$2 M$ & IM CENTER IM & \\
W & $W$ & LINE & E &
\end{tabular}

HE IGHT

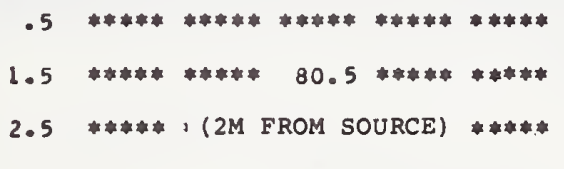

* HEST SIDE*

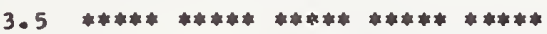

EAST SIDE*

MIC

*TOP*

HE IGHT $0.5 \quad 1.5 \quad 2.5 \quad 3.5$

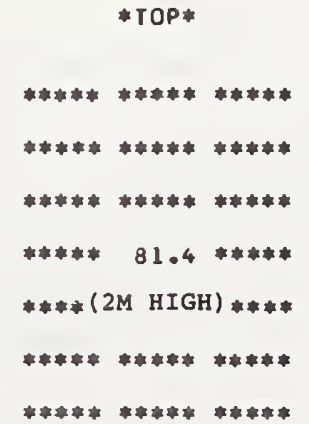

$\begin{array}{llll}3.5 & 2.5 & 1.5 & 0.5\end{array}$

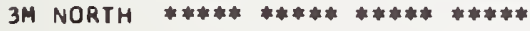

2M NORTH

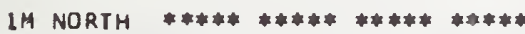

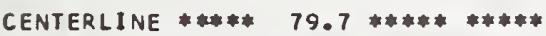

IM SOUTH $* *(2 M$ FROM SOURCE): $\neq *$

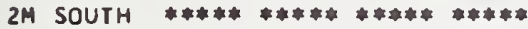

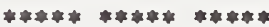

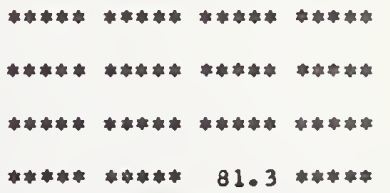

* (2M FROM SOURCE) *\#

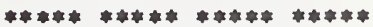

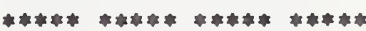

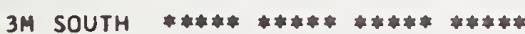

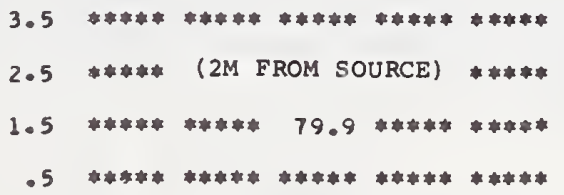




\begin{tabular}{|c|c|c|}
\hline ANGUL AR & DRIEI & TATION(OEG) \\
\hline $\begin{array}{l}\text { MIC HEIGHT } \\
\text { (ME TRES ) }\end{array}$ & NUM & $\begin{array}{l}\text { ELEVAT IUN } \\
\text { (DEG) }\end{array}$ \\
\hline .575 & 7 & 4.7 \\
\hline 1.725 & i & 14.4 \\
\hline 2.875 & 6 & 24.2 \\
\hline 4.025 & 2 & 35.2 \\
\hline 5.175 & 5 & 47.6 \\
\hline 6.375 & 3 & 65.7 \\
\hline 7.000 & 4 & 90.0 \\
\hline
\end{tabular}

\begin{tabular}{|c|c|c|c|c|c|c|c|c|c|c|c|}
\hline & & & & A & $=$ & Le & $(00)$ & & & & \\
\hline 0 & 30 & 60 & 90 & 120 & 150 & 180 & 210 & 240 & 270 & 300 & 330 \\
\hline $\begin{array}{l}61.3 \\
57.7 \\
59.6 \\
56.8 \\
62.5 \\
58.5 \\
68.0\end{array}$ & $\begin{array}{l}64.6 \\
65.5 \\
63.0 \\
61.3 \\
64.4 \\
55.8 \\
68.4\end{array}$ & $\begin{array}{l}60.2 \\
61.0 \\
59.9 \\
60.3 \\
63.3 \\
55.3 \\
68.0\end{array}$ & $\begin{array}{l}65.5 \\
62.1 \\
66.8 \\
52.6 \\
66.7 \\
57.4 \\
67.9\end{array}$ & $\begin{array}{l}66.4 \\
65.6 \\
68.1 \\
59.0 \\
69.7 \\
58.0 \\
68.1\end{array}$ & $\begin{array}{l}69.3 \\
67.8 \\
69.7 \\
62.2 \\
71.9 \\
57.2 \\
67.9\end{array}$ & $\begin{array}{l}72.2 \\
72.6 \\
73.5 \\
67.0 \\
73.8 \\
55.8 \\
68.6\end{array}$ & $\begin{array}{l}73.9 \\
74.2 \\
75.4 \\
69.0 \\
75.5 \\
55.3 \\
68.1\end{array}$ & $\begin{array}{l}73.0 \\
72.8 \\
75.1 \\
68.5 \\
74.3 \\
50.7 \\
67.7\end{array}$ & $\begin{array}{l}70.1 \\
68.5 \\
70.9 \\
63.8 \\
72.7 \\
56.6 \\
68.1\end{array}$ & $\begin{array}{l}67.6 \\
65.7 \\
68.2 \\
58.6 \\
70.8 \\
58.5 \\
67.4\end{array}$ & $\begin{array}{l}65.3 \\
63.5 \\
67.0 \\
56.9 \\
67.4 \\
56.4 \\
67.9\end{array}$ \\
\hline
\end{tabular}

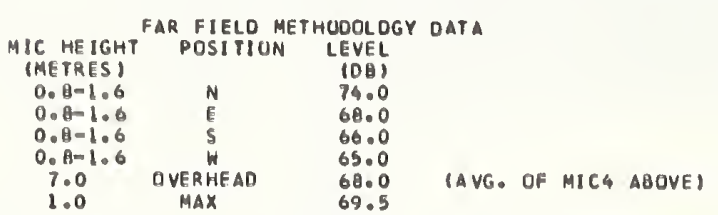

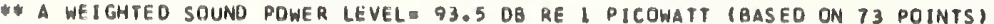

Table 3.20

A-WEIOHTED BOUND PRE8BURE LEVEL DATA

Test Number 20 Pure-Tone Loudspeaker Source, $1000 \mathrm{~Hz}$

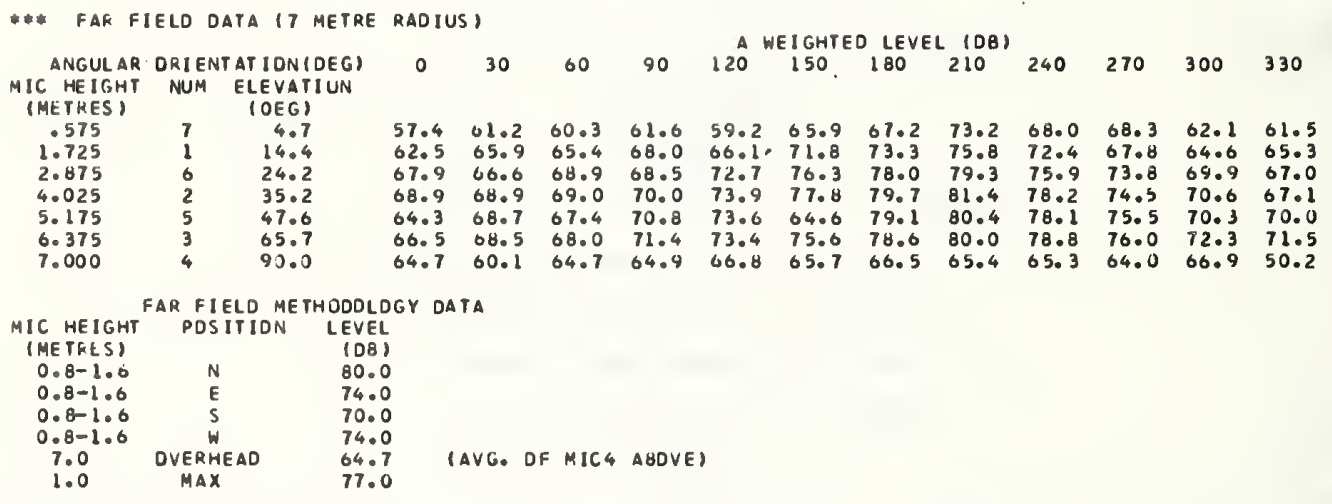

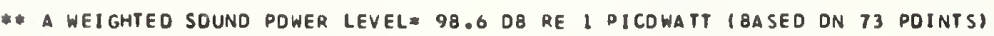

Table 3.21

A-WEIGHTED SOUND PRESSURE LEVEL DATA

Test Number $21 \quad$ Pure-Tone Loudspeaker Source, $2000 \mathrm{~Hz}$

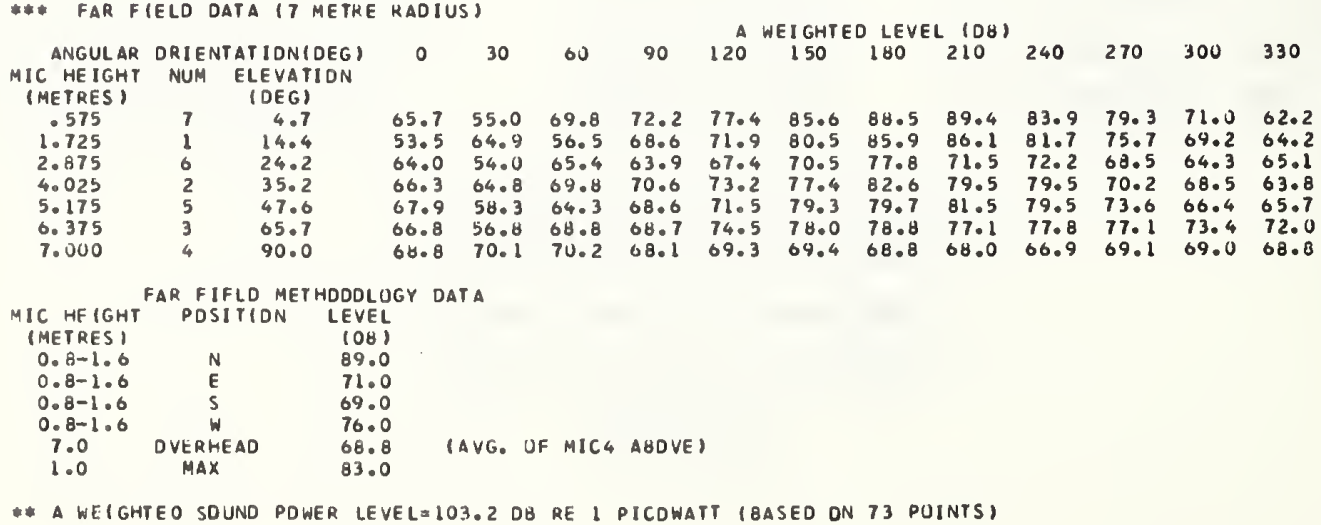




\section{SOUND POWER LEVEL CALCULATIONS}

\subsection{Far Field Sound Power Calculation Procedures}

The estimate of sound power $(W(f)$ ) from samples of mean square pressure on a measurement surface is of the form:

$$
W(f)=\sum_{i=1}^{n} \overline{p_{i}^{2}}(f) s_{I} / p c
$$

where $W(f)$ is the estimate of sound power (watts) at the frequency $f$

$\overline{p^{2}},(f)$ is the time-average squared pressure at position $i$ on the measurement surface at the frequency $f$ (pascals ${ }^{2}$ ).

$\overline{\left[p^{2}\right.}(f)=10^{\operatorname{SPL}}(i, f) / 10$ where $\overline{\operatorname{SPL}}(i, f)$ is the time average sound pressure level at position $i$ and frequency $f$.

$\mathrm{S}_{i}$ is the area associated with the ith measurement position (square metres).

$O C$ is the characteristic impedance of the medium ( $\rho$ is the density $\mathrm{kg} / \mathrm{m}^{3}$, c is the velocity of sound $\mathrm{m} / \mathrm{sec}$ ).

In the event that the microphone positions are chosen to represent equal areas of the measurement surface, then equation 3.1 reduces to:

$$
W(f)=\frac{S}{p c n}\left(\sum_{i=1}^{n} \overline{p_{i}^{2}}(f)\right)
$$

where: $S$ is the total area of the measurement surface (square metres).

The far-field measurement array was designed with this latter equation in mind. At each location of the array, each microphone, except the top one, samples a sector of the hemisphere from $\phi-\Delta \phi$ to $\phi+$ $\Delta \phi$ and from $\mathrm{z}_{1}=\mathrm{z}_{0}-\frac{\Delta \mathrm{z}}{2}$ to $\mathrm{z}_{2}=\mathrm{z}_{0}+\frac{\Delta \mathrm{z}}{2}\left[\right.$ where $\Delta \phi=15^{\circ}, \Delta \mathrm{z}=1.15 \mathrm{~m}$. $]$. The area of a segment of a spherical surface between $z_{1}$ and $z_{2}$ is $2 \pi r \Delta z$; thus the area represented by
each microphone position is:

$$
\begin{aligned}
\Delta S_{i}= & 2 \pi(7.0)(1.15)(30 / 360) \\
& =4.2 \mathrm{~m}^{2}
\end{aligned}
$$

The area sampled by the top microphone is:

$$
\begin{aligned}
\Delta S_{73}= & 2 \pi r^{2}-m\left(\Delta S_{i}\right) \\
= & 4.3 \mathrm{~m}^{2}
\end{aligned}
$$

For the purpose of sound power calculations in this report, these areas were treated as equal; however, it should be noted that the areas involved are not identical in shape. This is a conceptual disadvantage, since it is not entirely clear that the sample from the center of a long narrow rectangular shaped area will be as representative of the average level as a point which is at the center of a circle of the same area. Such considerations are, however, beyond the scope of this study .

\subsubsection{Systematic Error in the Far Field Array}

The array chosen presents another difficulty for the measurement of sound power level, which has been previously noted by Baade[7]. This has to do with the fact that a compact (ka<<l, $k=$ wave number, $\mathrm{a}=$ source radius) omnidirectional source at a height above the ground plane produces an interference pattern on a hemisphere above the plane which in the far field approximation is of the form [7].

$$
p^{2}(r, z)=p_{0}^{2}\left(\frac{I}{R_{I}^{2}}+\frac{1}{R_{2}^{2}}+\frac{2 R(\tau)}{R_{I} R_{2}}\right)
$$

where:

$$
\begin{aligned}
& p^{2}(r, z) \text { is the mean square pressure at helght } z \text { on a hemisphere } \\
& \text { centered over the sources, of radius } r \text {; }
\end{aligned}
$$


${ }^{2}{ }^{2}$ I at如

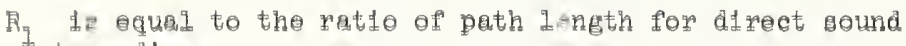
to radus s;

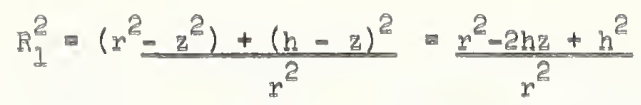

$R_{2} 1$ s equal to the ratio of path length for reflected sound to radius $r$;

$$
R_{2}^{2}=\frac{\left(r^{2}-z^{2}\right)+(h+z)^{2}}{r^{2}}=\frac{r^{2}+2 h z+h^{2}}{r^{2}}
$$

h 1 the height of the source center above the ground plane:

$R(\tau)$ is the auto correlation function of the source radiation.

For random noise;

$R(\tau)=\cos \left(2 \pi f_{0} \tau\right) \frac{\operatorname{Sin}(2 \pi \Delta f \tau)}{2 \pi \Delta f \tau}$

$f_{0}$ is the center frequency of the band, $\mathrm{Hz}$;

$\Delta f$ is the narrower bandwidth of the signal or the analyzing filter;

$\tau$ is the time delay between direct and reflected sound $\left[\tau=r\left(R_{1}-R_{2}\right) / c\right]$

$c=$ speed of sound in the propagation medium;

For pure tones, $\Delta f=0$ and $\frac{\sin x}{x}=1$ when $x=0$

so, $R(\tau)=\cos 2 \pi f \tau=\cos \left(2 \pi f r\left(R_{1}-R_{2}\right) / c\right)$. For $r^{2} / h z \gg 1$,

$R_{1} / R_{2} \quad 1$, and $f r\left(R_{1}-R_{2}\right) / c \quad 2 f h z / r c$.

For tones or bands of noise this expression predicts minime in the sound fleld at helghts which are odd multiples of fhz/re when $r^{2} \gg h z$.

Thus for a small fixed height source, superimposed on its inherent directivity, interference minima will occur at microphone heights $\mathrm{z}$ which are multiples of odd integers due to the $n$ ground plane reflection. Fortunately, our microphone array is sufficiently closely spaced so that minime will not occur at all microphones simultaneously, for a one metre source helght, unless the conditions $f \geq 1000 \mathrm{~Hz}$ and source radius much less then $0.053 \mathrm{~m}$ are met. At lower frequencies, the fect that maxima will occur at some microphone positions which will compensate, to some degree, for minima at other locations, presumably will tend to minimize this systematic error. Furthermore, for this description to apply to a minimum at the top microphone only, the frequency must be greater than about $300 \mathrm{~Hz}$ with a source radius much less than $0.16 \mathrm{~m}$. Thus, the fact that the typical component source size of a compressor is considerably larger than these dimensions, except possibly for engine exhaust, suggests that the existence of interference minima should not create a serious measurement problem for this array.

An estimate of the possible error in the far field measurement for an omnidrectional source can be obtained from $\mathrm{Eq} .3 .3$ as follows.

We note, that an estimate of sound power output without sampling error, for a fixed source helght is given by

$3 /$ We note that while the true power is analytically available by simpler means, at this juncture our purpose is to evaluate only the sampling error, uncluttered by other error sources. 
SYSTEMATIC ERROR IN ESTIMATION OF SOUNO POWER FROA A CUMPACT SOURGE USING SPL VALUES FRON A 73 POINT AKHAT ON A 7 METER HEMISPHERE

SOURCE - PURE TONE AT $1 / 3$ UB CENTER FREQUENCY

10 LOG NIESTIMATEOIIH

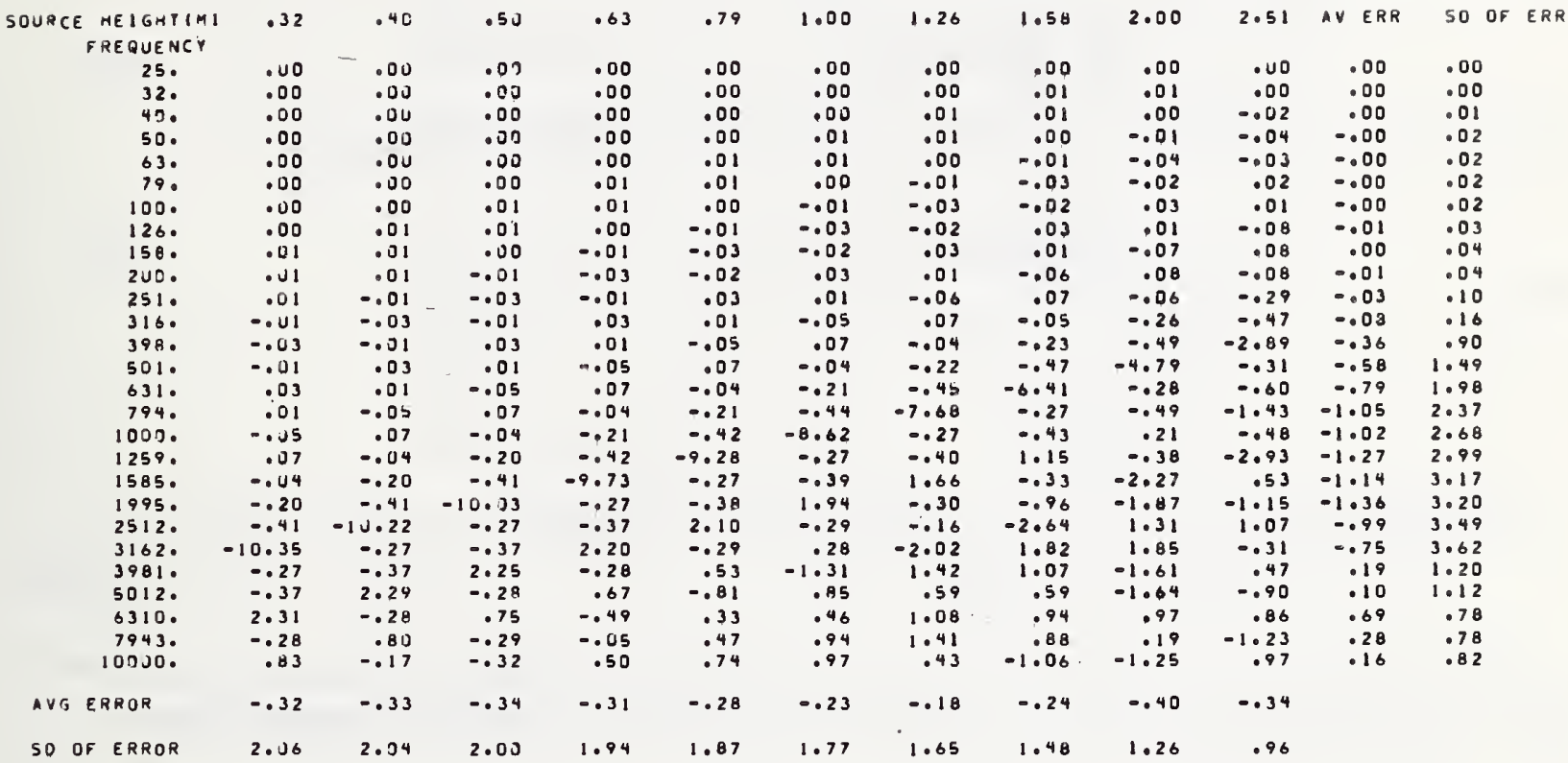

SYSTFMATIC ERROR IN ESTIMATION OF SUUNU POEER FROM A COMPACT SUURCE USING SPL VALUES FROM 73 POINT ARKAY ON A 7 METEH HEMISPHERE

SUURCE-PIINK NUISE $1 / 30.8$. WIOE AT $1 / 30.8$. CENTER FREUUENCY
10 LOG WIESTIMATEOI/

\begin{tabular}{|c|c|c|c|c|c|c|c|c|c|c|c|}
\hline SOURCE & $\begin{array}{l}\text { HEIGHTIM) } \\
\text { FKEQUENCY }\end{array}$ & .32 & .40 & . 5 ن & .63 & .79 & 1.00 & 1.26 & 1.58 & 2.00 & 2.51 \\
\hline & 25 & . UC & . u & .00 & .00 & .00 & .00 & .00 & .00 & .00 & .00 \\
\hline & 32. & כ0. & .04 & כט. & .00 & .00 & .00 & .00 & .01 & .01 &. .00 \\
\hline & 40. & . UD & .00 & .00 & .00 & .00 & .00 & .01 & .01 & .00 &. .02 \\
\hline & $\begin{array}{l}50 . \\
63 . \\
79 .\end{array}$ & ס סטי & $\begin{array}{l}.00 \\
.0 U \\
.0 L\end{array}$ & $\begin{array}{l}.03 \\
.00 \\
.00\end{array}$ & $\begin{array}{l}.01 \\
.00 \\
.01\end{array}$ & $\begin{array}{ll}.01 & 0 \\
.01 \\
.01\end{array}$ & $\begin{array}{l}.01 \\
.01 \\
.01\end{array}$ & $\begin{array}{r}.01 \\
.00 \\
-.01\end{array}$ & $\begin{array}{r}.00 \\
\therefore 01 \\
\therefore .03\end{array}$ & $\begin{array}{l}\because .01 \\
\because .03 \\
-.02\end{array}$ & $\begin{array}{r}-.04 \\
\because 02 \\
.01\end{array}$ \\
\hline & 100. & . U & .00 & .51 & .01 & .00 & $=.01$ & -.02 & -.01 & .02 & .001 \\
\hline & 126. & .00 & .01 & .01 & Dט. &. .01 & .0 .02 &. .01 & .02 & .01 & .001 \\
\hline & 158. & .01 & .01 & .00 & -.01 & .002 & .01 & .02 & -.00 & -.01 & .0 .02 \\
\hline & 200. & .01 & .00 & $-\cdot c t$ & -.02 & -.01 & .02 & -.00 & .001 & -.02 & .01 \\
\hline & 251. & .40 &. .01 &. .02 & .001 & .32 & -.00 & .000 &. .01 & .01 & .06 \\
\hline & 316. & $\because 01$ & -.02 & .001 & .02 & -.00 & .00 & .01 & .01 & .05 & .16 \\
\hline & 399. & -.02 &. .01 & .32 &. .00 & .00 & -.01 & .01 & .04 & .11 & .082 \\
\hline & $\begin{array}{l}5 \mathrm{J1} \text {. } \\
631 .\end{array}$ & $\begin{array}{r}-.01 \\
.42\end{array}$ & $\begin{array}{r}.02 \\
-.00\end{array}$ & $\begin{array}{l}\because .00 \\
-.00\end{array}$ & $\begin{array}{l}=.100 \\
\therefore .01\end{array}$ & $\begin{array}{r}.0 \\
.01 \\
.01\end{array}$ & $\begin{array}{l}.01 \\
.04\end{array}$ & $\begin{array}{l}.04 \\
.00\end{array}$ & $\begin{array}{r}.05 \\
.086\end{array}$ & $\begin{array}{l}-.85 \\
-.72\end{array}$ & $\begin{array}{l}-.73 \\
.002\end{array}$ \\
\hline & 794. & - • vo & -.00 &. .01 & .01 & .04 &. .03 & -.87 & -.70 & .006 & .33 \\
\hline & 1000. &.$- \Delta u$ &. .01 & .01 & .64 & -.05 &. .88 & -.68 & -.08 & .35 & .31 \\
\hline & $\begin{array}{l}1259 . \\
1585 .\end{array}$ & $\begin{array}{r}-.41 \\
.41\end{array}$ & $\begin{array}{l}.01 \\
.03\end{array}$ & $\begin{array}{r}.03 \\
-.08\end{array}$ & $\begin{array}{l}=07 \\
-.89\end{array}$ & $\begin{array}{l}=.89 \\
=.66\end{array}$ & $\begin{array}{l}9.67 \\
\therefore .08\end{array}$ & $\begin{array}{r}-.08 \\
.36\end{array}$ & $\begin{array}{r}.36 \\
.21\end{array}$ & $\begin{array}{r}.28 \\
-.25\end{array}$ & $\begin{array}{l}=.28 \\
=.04\end{array}$ \\
\hline & $\begin{array}{l}1995 . \\
2512 .\end{array}$ & $\begin{array}{r}.03 \\
-.09\end{array}$ & $\begin{array}{l}-.08 \\
-.89\end{array}$ & $\begin{array}{l}=.89 \\
=.65\end{array}$ & $\begin{array}{l}-.65 \\
-.07\end{array}$ & $\begin{array}{r}-.07 \\
.07\end{array}$ & $\begin{array}{r}.37 \\
.12\end{array}$ & $\begin{array}{r}.16 \\
\therefore .24\end{array}$ & $\begin{array}{r}-.24 \\
.02\end{array}$ & $\begin{array}{l}-.01 \\
-.03\end{array}$ & $\begin{array}{r}.01 \\
-011\end{array}$ \\
\hline & $\begin{array}{l}3162 . \\
3981 .\end{array}$ & $\begin{array}{l}-.89 \\
=.64\end{array}$ & $\begin{array}{l}-.64 \\
-.06\end{array}$ & $\begin{array}{r}-.16 \\
.17\end{array}$ & $\begin{array}{r}.37 \\
.08\end{array}$ & $\begin{array}{r}.09 \\
-.24\end{array}$ & $\begin{array}{r}-.24 \\
.07\end{array}$ & $\begin{array}{l}.05 \\
.07\end{array}$ & $\begin{array}{r}.01 \\
-.14\end{array}$ & $\begin{array}{r}=.15 \\
.02\end{array}$ & $\begin{array}{r}.00 \\
.004\end{array}$ \\
\hline & 5012. & $\therefore 06$ & .37 & .07 & -.24 & .09 & .11 & -.13 & .02 & .01 & .03 \\
\hline & 6310. & .37 & .26 & -.24 & .10 & .13 & -.13 & - . U & .002 & .05 & .02 \\
\hline & 7943. & .06 & -.25 & .11 & .14 & .13 &. .02 & .01 & .06 & .04 & .01 \\
\hline & 10000. & -.25 & .12 & .15 & -.13 & $=.04$ & .04 & .02 & .05 & -.04 & .01 \\
\hline AVs & ERHOR & -.05 & -.05 & -.04 & -.05 & -.05 & -.05 & .005 & -.05 & .005 & .004 \\
\hline $5 \cap 0$ & OF ERROR & .23 & .23 & .23 & .23 & .23 & .23 & .23 & .24 & .24 & .24 \\
\hline
\end{tabular}




$$
W=\int_{0}^{r} \frac{(2 \pi r) p^{2}(r, z) d z}{\rho c} .
$$

The approximation obtained from the far-field array is

$$
W^{\prime \prime}=\frac{\left(2 \pi r^{2}\right)}{p C} \sum_{i=1}^{73} \frac{p^{2}\left(r, z_{1}, \theta_{i}\right)}{73}
$$

since $p^{2}\left(r, z_{i}, \theta_{1}\right)$ is independent of $\theta$ for an omnidirectional source, eq. 3.5 reduces to

$$
w \cdots=\frac{\left(2 \pi r^{2}\right)}{\rho c} \sum_{i=1}^{T} a_{i} p^{2}\left(r, z_{i}\right)
$$

where:

$$
\begin{aligned}
& a_{1}=\left\{\begin{array}{l}
12 / 73, i=1 \text { to } 6 \\
1 / 73,1=7
\end{array}\right. \\
& z_{1}=\frac{(21-1) \Delta h}{2}
\end{aligned}
$$

The error in sound power level due to sampling $\left(\Delta \mathrm{L}_{\mathrm{w}}(\mathrm{s})\right)$ is given by

$$
\Delta \mathrm{L}_{\mathrm{W}}(\mathrm{S})=10 \log _{10} \frac{W^{11}}{W^{\prime}}
$$

The results of this calculation, using eq. 3.3 for $\mathrm{p}^{2}(\mathrm{r}, \mathrm{z})$ considering ten different source heights for the far field array with the sources radiating either a pure tone or one-third octave bands of noise, are shown in Tables 4.1 and 4.2 .

\subsubsection{Far Field Sound Power Calculations}

In performing the sound power calculations, the significance of the ambient correction was retained in terms of a signal quality code. As noted in section 2.3.5, the average sound pressure level value at a measurement position in each frequency band was coded to indicate one of the following types of data quality:

a) normal data (greater than $3 \mathrm{~dB}$ above ambient);

b) upper bound data (data within 0 to $3 \mathrm{~dB}$ above ambient, and corrected for ambient);

c) no useful data (data at or below ambient, or at or below instrument base line). Data value replaced by higher of ambient level minus $3 \mathrm{~dB}$ or baseline level.

In using these data in a sound power level calculation, the first step is the computation of an average sound pressure. The data were summed in three components of the average with a breakdown similar to the above, i.e.
a) sum of normal data, and number of points
b) sum of upper bound data, and number of points
c) sum of no-useful-data values, and number of points of no useful data.

The average sound pressure was determined by adding these sums together and dividing by the total number of points. The power level computed using this average sound pressure was assigned a quelity code according to the relative magnitude of these sums and the number of points involved as described below: 


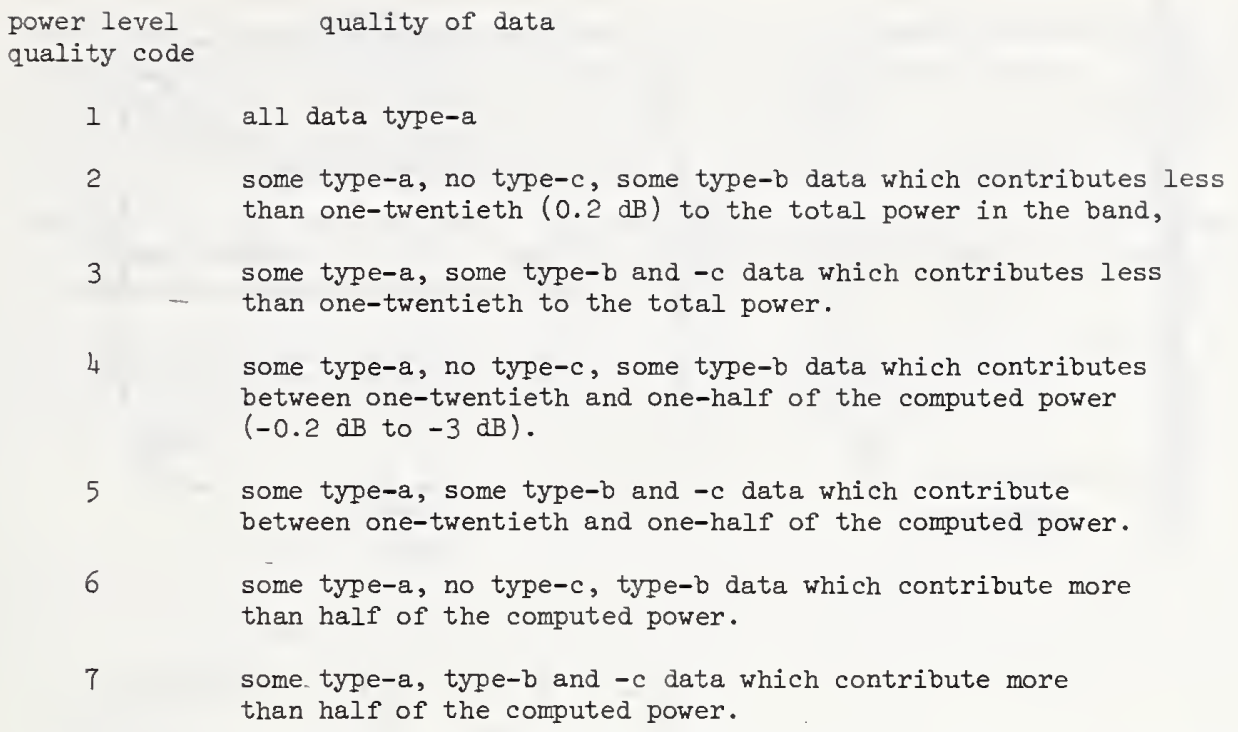

Investigation of the data indicated that in the frequency range $25 \mathrm{~Hz}$ to $10 \mathrm{kHz}$, there was always some type-a data so the above classification is complete. The data of code 4 through 7 are upper bound sound power levels, since the estimate of true power level will be less than calculated.

Data of code 1, 2, and 3 was further classified according to the magnitude of the pooled value of temporal variance according to the following scheme:

a. If the variance of the signal is less than that of electrical noise at the $99 \%$ level of confidence, then the signal is called tone-like.

b. If the variance of the signal is within the $99 \%$ confidence interval of electrical pink noise, the signal is called noise-like.

c. If the variance of the signal is significantly greater than that of electrical pink noise at the $99 \%$ level of confidence, the signal is called fluctuating.

The data in each band where the quality code is less than or equal to 3 is given a letter code T, $\mathbb{N}$ or $F$ corresponding to the above classification -- tone-like, noise-like and fluctuating, respectively:

\subsection{Near Field Sound Power Calculation Procedures}

The same general and specific forms of the far-field sound power calculations (eq. 3.1 and 3.2 ) also apply to the near-field calculations. The same order of magnitude sound power value will result since the value for mean square sound pressure will increase nominally inversely in proportion to the change in $S$ (as a consequence of the inverse square law of sound field spreading).

The calculations reported here were performed for two different measurement surfaces and three different subsets of the measured data as follows:

\section{Near-Field Calculations}

\section{Label Calculation Procedure}

NF 1 Conformal surface $4 /$ (defined by Fig. 8) - all appropriate measurement positions (see below).

NF 2 Conformal surface - engineering method, 8 measurement positions.

NF 3 Conformal surface - survey method, 5 measurement positions

NF 6 Rectangular surface - all measurement positions.

$4 /$ The conformal surface of radius $r$ is that surface which is defined by being everywhere a distance $r$ from the nearest point on the envelope of the principal radiating surfaces of the source. (See Figure 8). 

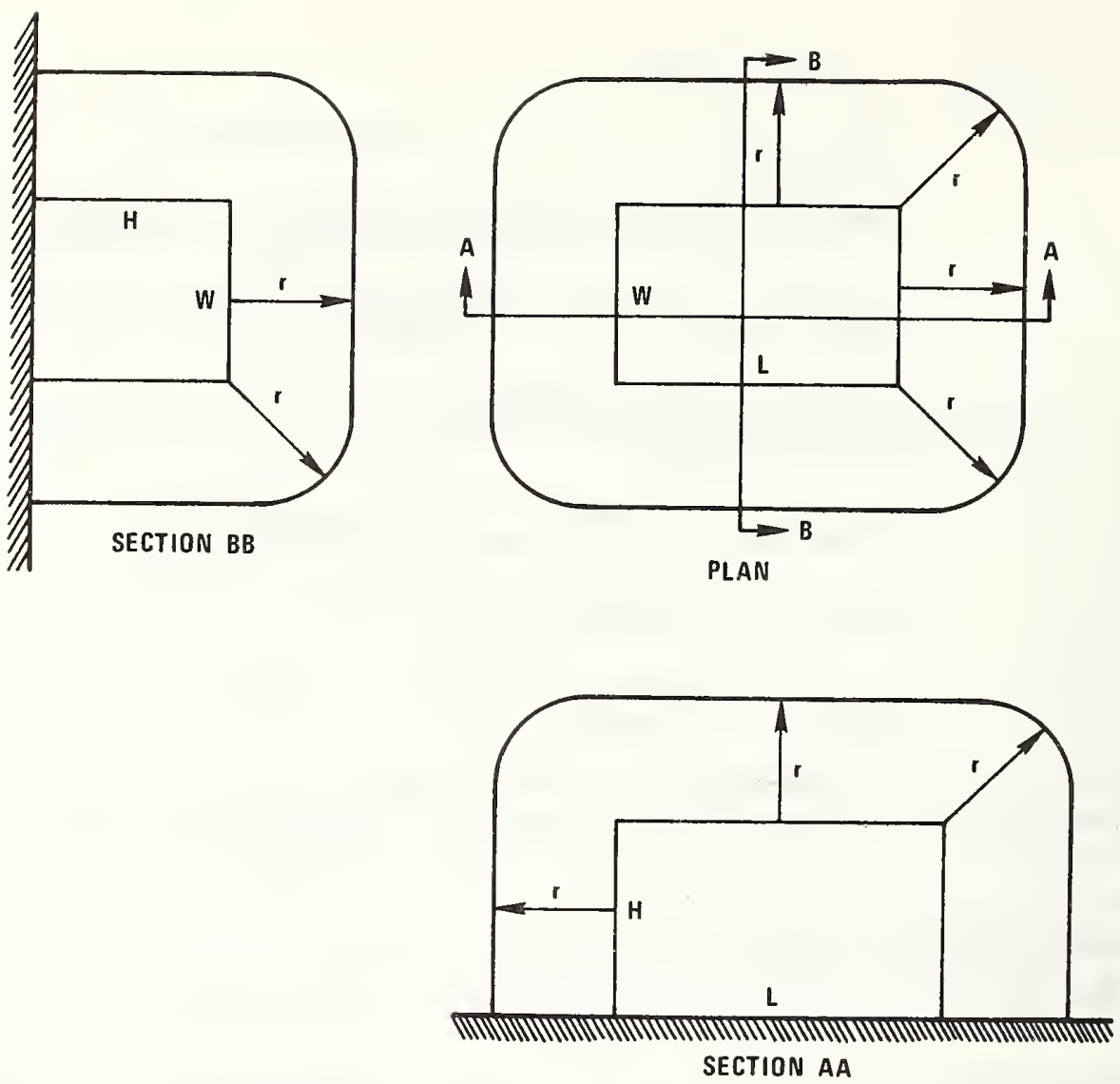

Figure 8 Conformal surface at a distance $r$ from a rectangular box

NF 7 Rectangular surface - engineering method, 9 measurement positions.

NF 8 Rectangular surface - survey method, 5 measurement positions.

The label above refers to the label of the calculation procedure as it appears in Tables $5.1-5.17$ and Table 6. The references to engineering and survey methods refer to Iso Draft International Standards[2] For Sound Power Eevel Determination (DIS 3744 and DIS 3746, respectively). The data were taken on a rectangular measurement surface (e.g., with square corners) rather than a conformal surface, therefore some modification of the data set was required for the conformal surface calculations. The modifications were as follows:

NF-1 - Data points near edges and corners were included if the data points were with the surface's corresponding distance from the source surface $r_{s}$ such that:

$S_{C}(1) \leq S_{C}\left(r_{S}\right) \leq 1.125 S_{C}(I)$
$\left(S_{C}(r)=2 h(l+w+\pi r)+\ell w+\pi r(l+w)+2 \pi r^{2}\right)$

This results in retaining some measurement positions (near edges and corners) which would be

$5 /$ Since all of the compressors in this study were contained within rectangular shaped enclosures, the envelope taken for the source was also rectangular in shape, and of dimensions $l, w$, $h$ where $l$ and $w$ are the length and width of the enclosure respectively, and $h$ is the height of the top of the enclosure above the reflecting plane. 
as much as $0.5 \mathrm{~dB}$ below the the expected value if inverse square spreading applies on the measurement surface. The actual number of measurement positions used in each NF-l calculation are identified in the data tables. It should be noted that most of the measurement positions do lie on the measurement surface. An estimate of the maximum downward bias

$(\Delta L(P))$ introduced by including those points not on the measurement surface is $-0.2 d B \leq \Delta L$ $(P) \leq 0$, with the maximum error decreasing as source size increases.

NF-2 - The ISO draft standard engineering method prescribes an eight point measurement array for use with a conformal surface. These eight points have position corrdinates as follows (for a source of dimension $\ell, w, h$ ):

\begin{tabular}{c|c|c|c} 
Pos. No. & $x$ & $y$ & $z^{*}$ \\
\hline 1 & $a$ & 0 & $h_{1}$ \\
2 & 0 & $b$ & $h_{1}$ \\
3 & $-a$ & $h_{1}$ \\
4 & 0 & $-b$ & $h_{1}$ \\
5 & $a / 2$ & $b_{1}$ & $h_{2}$ \\
6 & $-a / 2$ & $b_{1}$ & $h_{2}$ \\
7 & $-a / 2$ & $-b_{1}$ & $h_{2}$ \\
8 & $a / 2$ & $-b_{1}$ & $h_{2}$
\end{tabular}

Where: $a=1 / 2 \ell+r$

$b=1 / 2 w+r$

$c=h+r$

$h_{1}=1 / 4(b+c-r)$

$h_{2}=3 / 4(b+c-r) \leq c$

$b_{1}=1 / 2(b+c-r) \leq b$

* The origin of the coordinate system is on the reflecting plane under the center of the compressor.

The approximation of these positions used in the calculation in this study are:

positions $1-4$ ( $x, y$ dimensions as indicated, $h_{1}$ nearest half integral value in metres to calculated value)

positions 5-8 ( $\mathrm{x}, \mathrm{y}$ dimensions, nearest integral values to calculated value. $\left.h_{2}=c\right)$.

NF-3 \& -8 The five positions used for both calculations are the positions $1.5 \mathrm{~m}$ high, at the center of each side, and the position on the center of the top array. The only difference in the calculation is the difference in the area $S$ used for the measurement surface.

$\mathrm{NF}-6$

All points and rectangular surface used.

NF-7 The nine points required for this calculation include the five positions used in NF-3 and -8 plus four additional points at the corners of the measurement surfaces. These four points were approximated by the nearest measurement points.

The effect of these approximations may be treated as equivalent to a positioning error. The actual error introduced is a function of the directivity of the sound source -- most of the positioning error is a lateral displacement on the measurement surface, as opposed to a "radial" displacement off the measurement surface. Therefore, the approximations, in general, represent non-biasing errors which may or may not contribute to the imprecision of the measurement, but will not tend to produce systematic bias. 


\subsection{Sound Power Level Data}

The results of the sound power level calculations for the 17 compressors are presented in Tables 5.1 to 5.17. Each table consists of five parts including: a plot of far-field power level, a table of signal quality for far-field and near-field data, a table of far- and near-field power levels and differences between near- and far-field power levels, and a table of far-field methodology power levels and the difference from far-field power level.

The top of the table is a plot of the $1 / 3$ octave band, far-field sound power level vs frequency. Underneath the frequency scale is the signal quality code for both far-field and near-field (NF type 6) sound power level data using the same frequency scale as the plot. The next entry in the table is the sound power level data for far-field and six different near- field calculations as described in Sections 4.1 and 4.2. The data are presented in a two line format with the first line containing A-weighted and linear values plus the one-third octave band sound power level for band center frequencies from $25 \mathrm{~Hz}$ to $400 \mathrm{~Hz}$. The second line of each entry is the one-third octave band sound power level for band center frequencies from $500 \mathrm{~Hz}$ to $10,000 \mathrm{~Hz}$.

The second group of entries in the data table give the deviations of the various near-field calculation procedures from the far-field sound power level. The sign convention is such that positive values imply that the near-field power level is higher than the far-field power level. The significance of large individual deviations at low frequencies should be evaluated in the context of the magnitude of the signal quality factor, and the fact that large values of this factor (greater than 3) indicates a serious ambient noise problem (which typically caused an over estimate of the far field power level).

The far-field methodology data is presented in octave bands, and A- and C- weighted levels since the data were recorded in this format. For comparison purposes, the octave band and C-weighted far-field power level were computed from the one-third octave band data. 


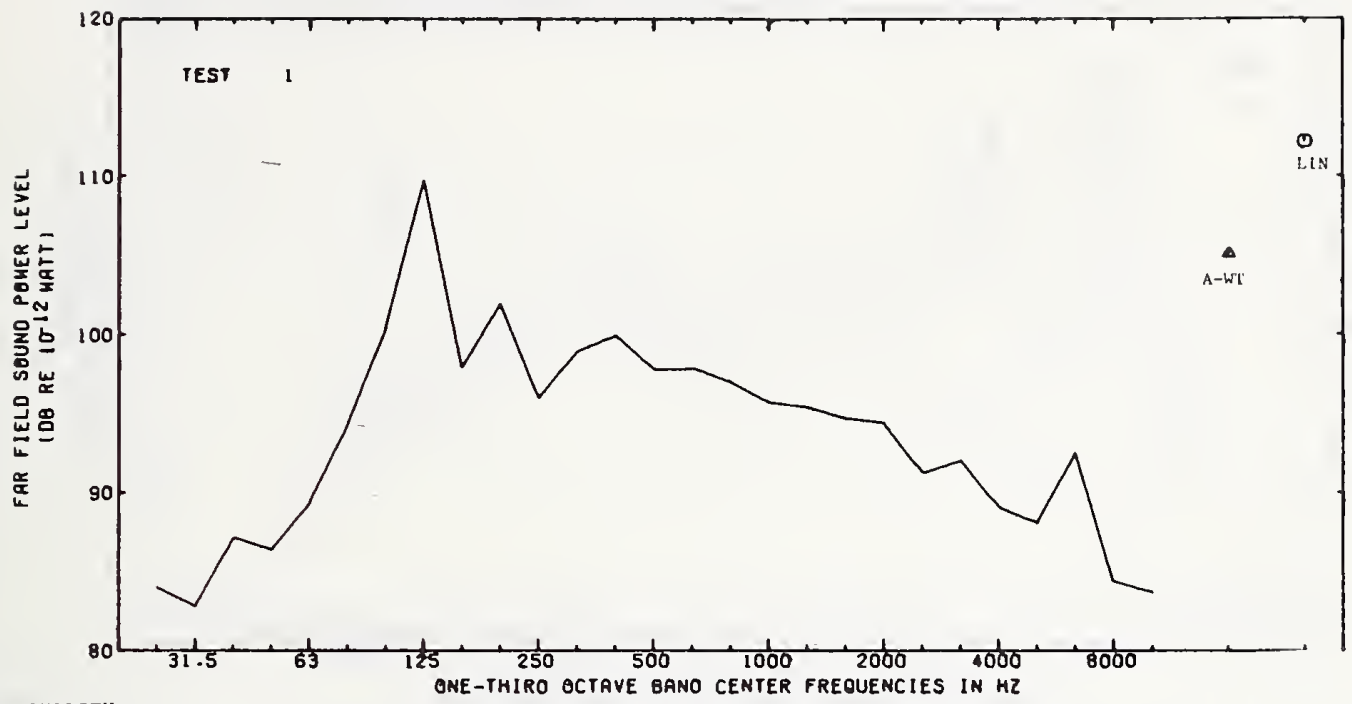

SIGNAL QUALITY

$\begin{array}{llllllllllllllllllllllllllllll}\text { FAR FIELO } & 7 & 7 & 4 & 5 & \text { F } & \text { F } & \text { F } & \text { T } & \text { F } & \text { F } & \text { N } & \text { F } & \text { F } & \text { F } & \text { F } & \text { F } & \text { F } & \text { F } & \text { F } & \text { F } & \text { F } & \text { F } & \text { F } & \text { F } & \text { F } & \text { F } & \text { F } \\ \text { NEAR FIELO } & \text { F } & \text { N } & \text { N } & \text { N } & \text { N } & \text { N } & \text { T } & \text { T } & \text { N } & \text { N } & \text { N } & \text { N } & \text { N } & \text { F } & \text { N } & \text { F } & \text { N } & \text { F } & \text { F } & \text { F } & \text { F } & \text { F } & \text { F } & \text { F } & \text { F } & \text { F } & \text { F }\end{array}$

IEST NUMAER 1

CIIMPRE SSOR SURFACE AREA=11.02 SO. METRES

34 NEAR FIELO MIIC PUSITIIONS.OF WHICH 33 USED IN NFI

WE IGHI ING- LOW FREOUENCY
HIGH FRFOUFNCY
TAR FIELD PWL
NF PHL 1
NF PWL 2
NF PWL 3
NF PWL 6
NF PWL 7
NF PWL 8

NF PWL 1

NF P WL 2

NF PWL 3

NF PWL 6

NF PWL 7

NF PWL a

FAR FIELO PWL

FAR FIFLD METHOOOLOGY PHL. OEVIATIOUNS OF FF MFTH FFIMA FF

\begin{abstract}
WE IGHTEU SUUNO POHER LEVELIOB RE I PICOWATT)

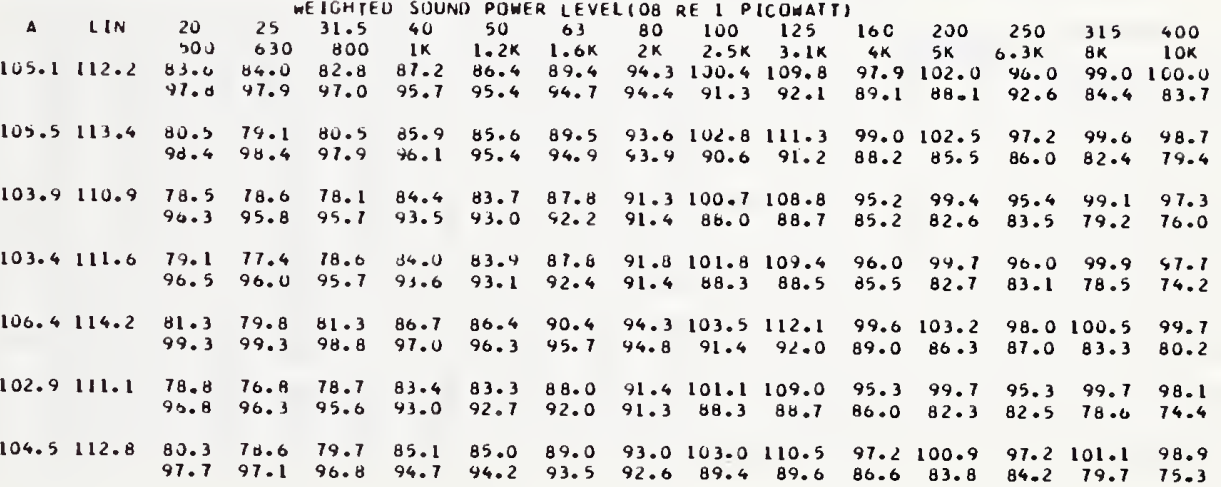

OEVIATIONS FROM FF PWLIDBI

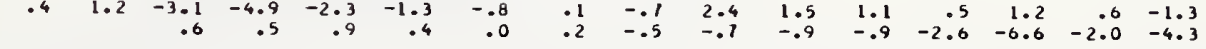

$\begin{array}{rrrrrrrrrrrrrrrr}-1.2 & -1.3 & -5.1 & -5.4 & -4.7 & -2.8 & -2.7 & -1.6 & -3.0 & .3 & -1.0 & -2.7 & -2.6 & -.06 & .1 & -2.7 \\ & & -1.5 & -2.1 & -1.3 & -2.2 & -2.4 & -2.5 & -3.0 & -3.3 & -3.4 & -3.9 & -5.5 & -9.1 & -5.2 & -7.7\end{array}$

$\begin{array}{llllllllllllllll}-1.7 & -.6 & -4.5 & -6.3 & -4.2 & -3.2 & -2.5 & -1.6 & -2.5 & 1.4 & -.4 & -1.9 & -2.3 & -.0 & .9 & -2.3\end{array}$

$\begin{array}{llllllllllllll} & -2.3 & -2.3 & -3.0 & -3.0 & -3.6 & -3.6 & -5.4 & -9.5 & -5.9 & -9.5\end{array}$

$\begin{array}{rrrrrrrrrrrrrrrr}1.3 & 2.0 & -2.3 & -4.2 & -1.5 & -.5 & .0 & 1.0 & -.0 & 3.1 & 2.3 & 1.7 & 1.2 & 2.0 & 1.5 & -.3 \\ & & 1.5 & 1.6 & 1.8 & 1.3 & .9 & 1.0 & .4 & .1 & -.1 & -.1 & -1.8 & -5.6 & -1.1 & -3.5\end{array}$

$\begin{array}{rrrrrrrrrrrrrrrr}-2.2 & -1.1 & -4.8 & -7.2 & -4.1 & -3.8 & -3.1 & -1.4 & -2.9 & .7 & -.8 & -2.0 & -2.3 & -.7 & .7 & -1.9 \\ & & -1.0 & -1.6 & -1.4 & -2.7 & -2.7 & -2.7 & -3.1 & -3.0 & -3.4 & -3.1 & -5.8 & -10.1 & -5.8 & -9.3\end{array}$

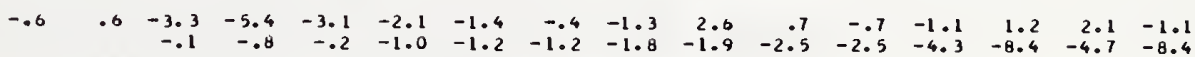

TABLE OF OCTAVE BANO PUWFR LEVELS

\begin{tabular}{rrrrrrrrrrr}
$\Delta$ & $C$ & 31.5 & \multicolumn{1}{c}{63} & 125 & 250 & 500 & $1 K$ & $2 K$ & $4 K$ & $8 K$ \\
105.1 & 112.8 & 89.9 & 96.0 & 110.5 & 104.4 & 103.5 & 100.9 & 98.5 & 94.9 & 93.7 \\
100.6 & 113.0 & .0 & 96.7 & 109.8 & 104.9 & 104.8 & 100.3 & 98.7 & 95.8 & 88.3 \\
1.5 & .2 & .0 & .7 & -.7 & .5 & 1.3 & -.6 & .2 & .9 & -5.4
\end{tabular}




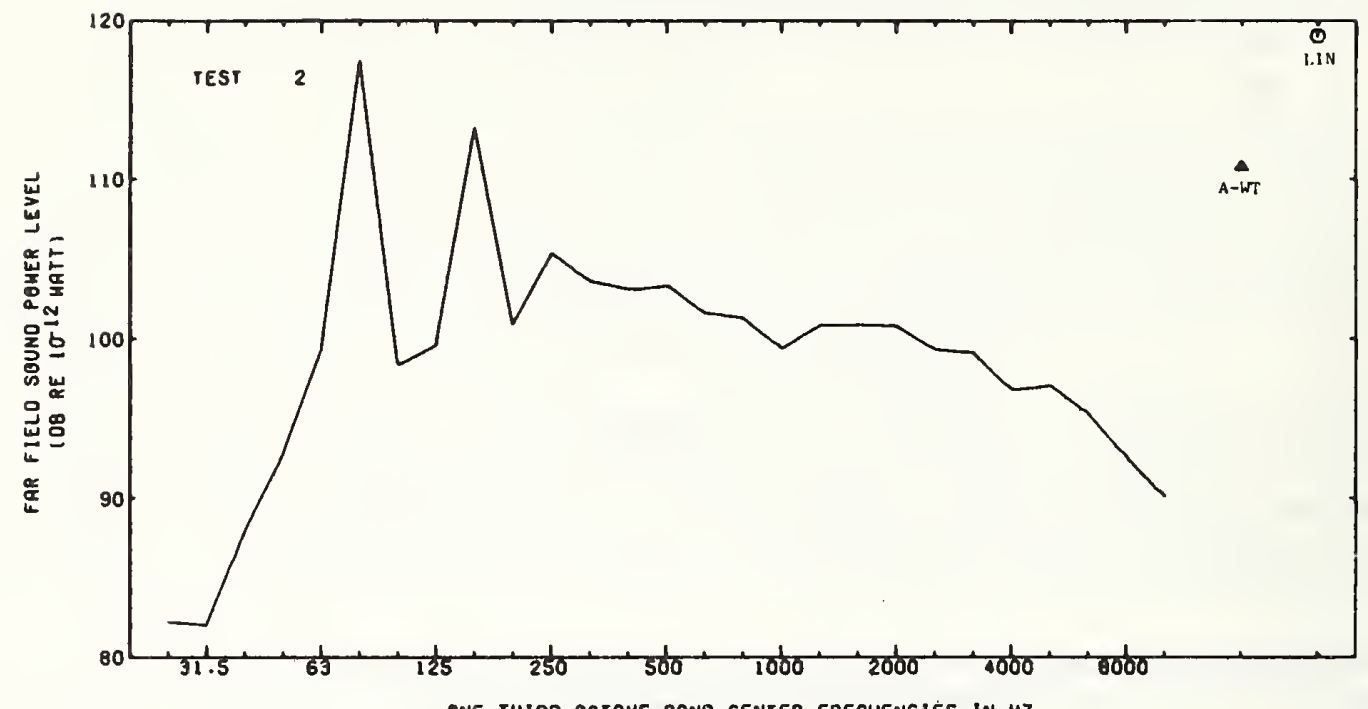

\section{$\begin{array}{llllllllllllllllllllllllllllll}\text { FAR FIELD } & 5 & 4 & \boldsymbol{F} & \mathbf{T} & \mathbf{T} & \mathbf{T} & \mathbf{N} & \mathbf{T} & \mathbf{T} & \mathbf{T} & \mathbf{T} & \mathbf{T} & \mathbf{N} & \mathbf{N} & \boldsymbol{F} & \boldsymbol{F} & \boldsymbol{F} & \boldsymbol{F} & \boldsymbol{F} & \boldsymbol{F} & \boldsymbol{F} & \boldsymbol{F} & \boldsymbol{F} & \boldsymbol{F} & \boldsymbol{F} & \boldsymbol{F} & \boldsymbol{F} \\ \text { NEAR FIELD } & \mathbf{N} & \mathbf{F} & \mathbf{N} & \mathbf{T} & \mathbf{T} & \mathbf{T} & \mathbf{T} & \mathbf{T} & \mathbf{T} & \mathbf{T} & \mathbf{T} & \mathbf{T} & \mathbf{N} & \mathbf{N} & \mathbf{N} & \boldsymbol{F} & \boldsymbol{F} & \boldsymbol{F} & \boldsymbol{F} & \boldsymbol{F} & \boldsymbol{F} & \boldsymbol{F} & \boldsymbol{F} & \boldsymbol{F} & \boldsymbol{F} & \boldsymbol{F} & \boldsymbol{F}\end{array}$ \\ TEST NUMBER 2 \\ 45 NEAR FIELO MIC POSITIONS, OF NHICH 33 USEO IN NFI \\ COMPRF SSOR SURFACE AREA $=8.68$ SO. METRES}

WE IGHT ING-LOH FRE QUENCY HIGH FREQUENCY

FAR FIELO PWL

NF PWL 1

NF PHL 2

NF PWL 3

NF PWL o

NF P WL 7

NF PHL 8

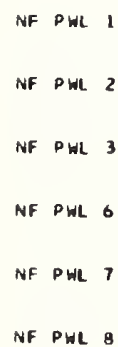

FAR FIELO PHL

FAR FIELO ME THOOOLOGY PHL OEVIATLONS OF FF METH FROM FF
HE IGHTEO SUUNO POHER LEVELIOQ RE I PICOHATII

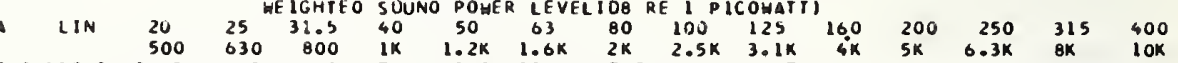
$110.8119 .0 \quad 02.3 \quad 02.2 \quad 82.0 \quad 87.4 \quad 92.8 \quad 99.4 \quad 117.5 \quad 98.4 \quad 99.7113 .3100 .9105 .4103 .6103 .1$ 103.4101 .6101 .399 .4100 .4100 .9100 .899 .399 .196 .897 .195 .392 .690 .1

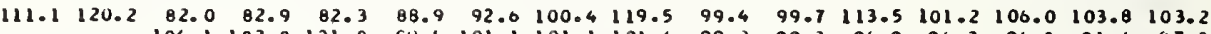
$104.1102 .0101 .8 \quad 94.3 \quad 101.1101 .1101 .499 .0 \quad 99.396 .996 .394 .891 .4 \quad 87.8$

$110.8114 .0 \quad 80.8 \quad 80.9 \quad 81.1 \quad 88.3 \quad 91.6 \quad 99.4 \quad 118.5 \quad 98.2 \quad 99.6 \quad 114.2 \quad 100.3 \quad 105.0102 .4102 .4$ $103.7102 .1 \quad 102.0949 .3101 .0101 .1101 .598 .498 .897 .196 .894 .591 .598 .6$

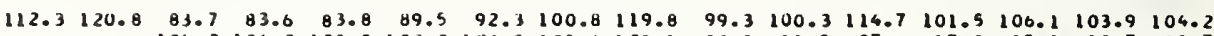
$106.2104 .0103 .0100 .0102 .8102 .4102 .1 \quad 98.999 .3 \quad 97.0 \quad 97.295 .892 .799 .7$

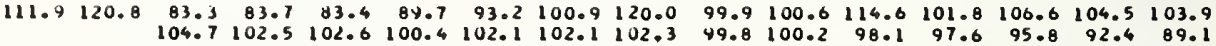

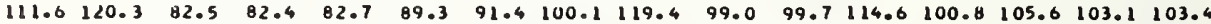
$105.1 \quad 103.2102 .3 \quad 99.8 \quad 102.0101 .7101 .4 \quad 99.099 .397 .6 \quad 96.695 .891 .999 .2$

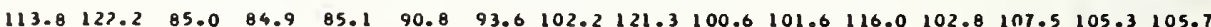
$107.7105 .5 \quad 104.4 \quad 101.5104 .4 \quad 103.9103 .6100 .2100 .699 .098 .697 .093 .990 .9$
\end{abstract}

\begin{tabular}{|c|c|c|c|c|c|c|c|c|c|c|c|c|c|c|c|}
\hline \multicolumn{16}{|c|}{ DEVIATIONS FROM FF PHLIOBI } \\
\hline .3 & 1.2 & $\begin{array}{r}-.3 \\
.7\end{array}$ & .7 & $\begin{array}{l}.3 \\
.5\end{array}$ & $\begin{array}{r}1.0 \\
.1\end{array}$ & $\begin{array}{r}-.2 \\
.2\end{array}$ & $\begin{array}{r}1.0 \\
.2\end{array}$ & $\begin{array}{r}2.0 \\
.6\end{array}$ & $\begin{array}{l}1.0 \\
-.3\end{array}$ & $\begin{array}{l}.0 \\
.2\end{array}$ & .2 & $\begin{array}{r}.3 \\
-.8\end{array}$ & $\begin{array}{r}.6 \\
-.5\end{array}$ & -1.2 & -2.3 \\
\hline .0 & .6 & $\begin{array}{r}-1.5 \\
.3\end{array}$ & $\begin{array}{r}-1.3 \\
.5\end{array}$ & $\begin{array}{r}-.9 \\
.7\end{array}$ & $\begin{array}{r}.4 \\
-.1\end{array}$ & $\begin{array}{r}-1.2 \\
.1\end{array}$ & $\begin{array}{r}-.0 \\
.2\end{array}$ & $\begin{array}{r}1.0 \\
.7\end{array}$ & $\begin{array}{l}-.2 \\
-.9\end{array}$ & $\begin{array}{l}-.1 \\
-.3\end{array}$ & $\begin{array}{l}.9 \\
.3\end{array}$ & $\begin{array}{l}-.6 \\
-.3\end{array}$ & $\begin{array}{l}-.4 \\
-.8\end{array}$ & $\begin{array}{l}-1.2 \\
-1.1\end{array}$ & $\begin{array}{r}-.7 \\
-1.5\end{array}$ \\
\hline 1.5 & 1.8 & $\begin{array}{l}1.4 \\
2.8\end{array}$ & $\begin{array}{l}1.4 \\
2.4\end{array}$ & $\begin{array}{l}1.8 \\
1.7\end{array}$ & $\begin{array}{r}1.6 \\
.6\end{array}$ & $\begin{array}{l}-.5 \\
1.9\end{array}$ & $\begin{array}{l}1.4 \\
1.5\end{array}$ & $\begin{array}{l}2.3 \\
1.3\end{array}$ & $\begin{array}{r}.9 \\
-.4\end{array}$ & $\begin{array}{l}.6 \\
.2\end{array}$ & $\begin{array}{r}1.4 \\
.8\end{array}$ & $\begin{array}{l}.6 \\
.1\end{array}$ & $\begin{array}{r}.7 \\
.5\end{array}$ & $\cdot 3$ & $\begin{array}{l}1.1 \\
-. .4\end{array}$ \\
\hline $1 \cdot 1$ & 1.8 & $\begin{array}{l}1.0 \\
1.3\end{array}$ & $\begin{array}{r}1.5 \\
.9\end{array}$ & $\begin{array}{l}1.4 \\
1.3\end{array}$ & $\begin{array}{l}1.8 \\
1.0\end{array}$ & 1.4 & $\begin{array}{l}1.5 \\
1.2\end{array}$ & $\begin{array}{l}2.5 \\
1.5\end{array}$ & $\begin{array}{r}1.5 \\
.5\end{array}$ & 1.9 & $\begin{array}{l}1.3 \\
1.3\end{array}$ & .9 & $\begin{array}{r}1.2 \\
.5\end{array}$ & $\begin{array}{r}.9 \\
-.2\end{array}$ & $\begin{array}{r}.8 \\
-1.0\end{array}$ \\
\hline .8 & 1.3 & .22 & $\begin{array}{r}.2 \\
1.6\end{array}$ & $\begin{array}{r}.7 \\
1.0\end{array}$ & $\begin{array}{r}1.4 \\
.4\end{array}$ & $\begin{array}{r}-1.4 \\
1.1\end{array}$ & $\begin{array}{l}.7 \\
.8\end{array}$ & $\begin{array}{r}1.9 \\
.6\end{array}$ & $\begin{array}{r}.6 \\
-.3\end{array}$ & $\begin{array}{r}.0 \\
.2\end{array}$ & $\begin{array}{r}1.3 \\
.8\end{array}$ & $\begin{array}{l}=.1 \\
=.5\end{array}$ & $\begin{array}{r}.2 \\
.5\end{array}$ & $\begin{array}{l}-.5 \\
-.7\end{array}$ & $\begin{array}{r}.3 \\
-.9\end{array}$ \\
\hline 3.0 & 3.2 & $\begin{array}{l}2.7 \\
4.3\end{array}$ & $\begin{array}{l}2.7 \\
3.9\end{array}$ & $\begin{array}{l}3.1 \\
3.1\end{array}$ & $\begin{array}{l}2.9 \\
2.1\end{array}$ & $\begin{array}{r}.8 \\
3.5\end{array}$ & $\begin{array}{l}2.8 \\
3.0\end{array}$ & $\begin{array}{l}3.6 \\
2.8\end{array}$ & $\begin{array}{r}2.2 \\
.9\end{array}$ & $\begin{array}{l}1.9 \\
1.5\end{array}$ & $\begin{array}{l}2.7 \\
2.2\end{array}$ & $\begin{array}{l}1.9 \\
1.5\end{array}$ & $\begin{array}{l}2.1 \\
1.7\end{array}$ & $\begin{array}{l}1.7 \\
1.3\end{array}$ & $\begin{array}{r}2.6 \\
.8\end{array}$ \\
\hline
\end{tabular}

TABLE DF OCTAVE BANO POHER LEVELS

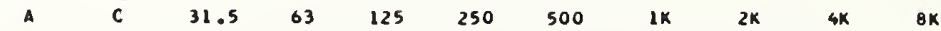

$\begin{array}{lllllllllll}110.8 & 119.7 & 89.7 & 117.6 & 113.6 & 108.4 & 107.5 & 105.4 & 105.2 & 102.6 & 97.9\end{array}$

$\begin{array}{lllllllllll}113.0 & 120.8 & .0 & 118.5 & 115.3 & 108.1 & 109.9 & 105.6 & 104.8 & 104.8 & 102.1\end{array}$

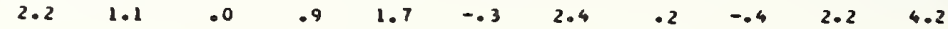




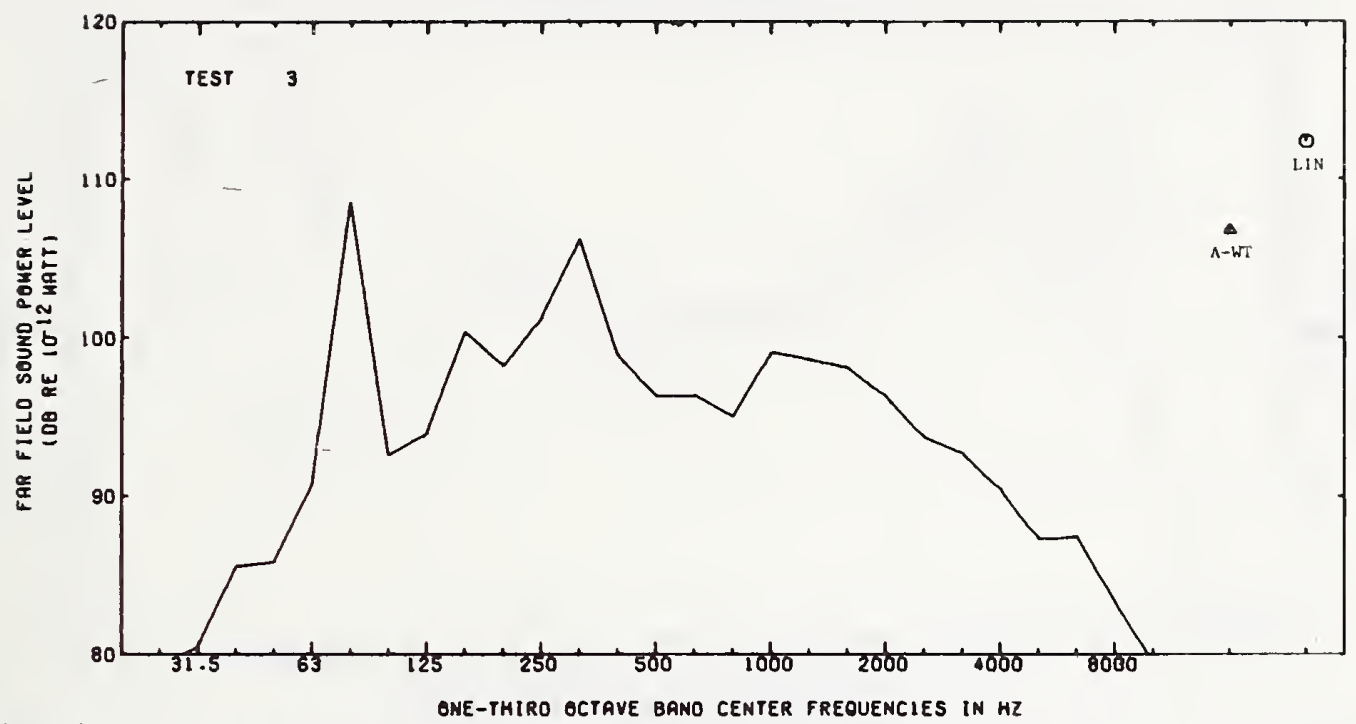

SIGNAL QUALITY

$\begin{array}{lllllllllllllllllllllllllllll}\text { FAR FIELD } & 7 & 4 & \text { F } & \mathbf{5} & \text { N } & \text { T } & \text { N } & \text { N } & \text { T } & \text { T } & \text { T } & \text { F } & \text { N } & \text { N } & \text { F } & \text { N } & \text { F } & \text { F } & \text { F } & \text { F } & \text { F } & \text { F } & \text { F } & \text { F } & \text { F } & \text { F } & \text { F } \\ \text { NEAR FIELD } & \text { F } & \text { F } & \text { N } & \text { N } & \text { T } & \text { T } & \text { N } & \text { N } & \text { T } & \text { T } & \text { T } & \text { F } & \text { T } & \text { N } & \text { F } & \text { N } & \text { F } & \text { F } & \text { F } & \text { F } & \text { F } & \text { F } & \text { F } & \text { F } & \text { F } & \text { F } & \text { F }\end{array}$

TEST NUMBER 3

57 NEAR FIELU MIC PUSITIONS, OF HHICH 41 USEO IN NFI

COMPRESSOR SURFACE AREA $=13.67$ SO. METRES

CONFORMAL SURFACE AREA $(R=1 M)=61.1350$. METRES

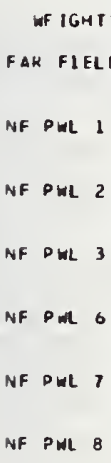

NF PUL,

NF PWL 2

NF PHL 3

NF PUL 6

NF PWL 7

NF PHL 8

FAR FIELO PWL

FAR FIELD METHODOLOGY PHL OEVIATIONS OF FF METH FROM FF

\begin{abstract}
A LIN TEIGHTEO SOUNO POWER LEVELIOB RE 1 PICOHATTI
$\begin{array}{lllllllllllllll}4 & 20 & 25 & 31.5 & 40 & 50 & 63 & 80 & 100 & 125 & 160 & 200 & 250 & 315 & 400 \\ & 500 & 630 & 800 & 1 K & 1.2 K & 1.6 K & 2 K & 2.5 K & 3.1 K & 4 K & 5 K & 6.3 K & 8 K & 10 K\end{array}$

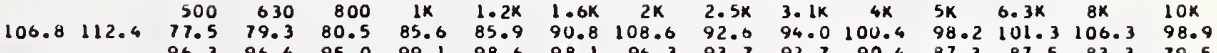

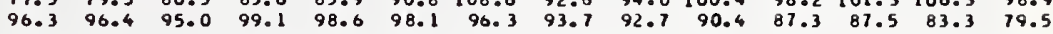

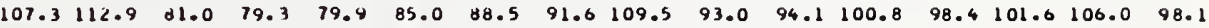

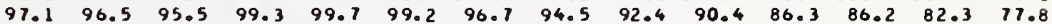

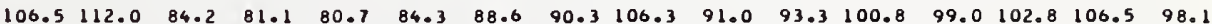

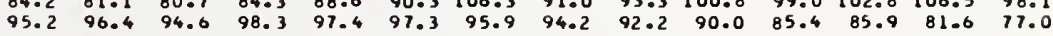

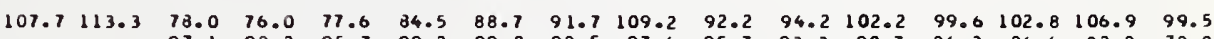

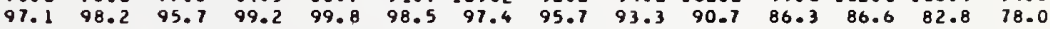

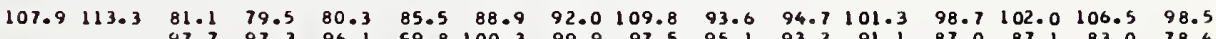
$\begin{array}{lllllllllllllll}97.7 & 97.3 & 96.1 & 99.8 & 100.3 & 99.9 & 97.5 & 95.1 & 93.2 & 91.1 & 87.0 & 87.1 & 83.0 & 78.4\end{array}$

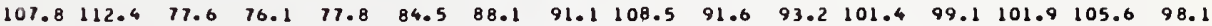

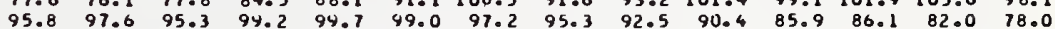

$108.9114 .7 \quad 79.2 \quad 77.3 \quad 78.9 \quad 85.7 \quad 89.9 \quad 93.0 \quad 110.7 \quad 93.5 \quad 95.4 \quad 103.5 \quad 100.9 \quad 104.2108 .3100 .9$

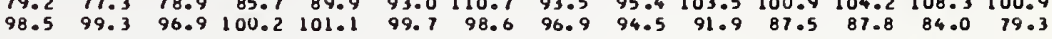

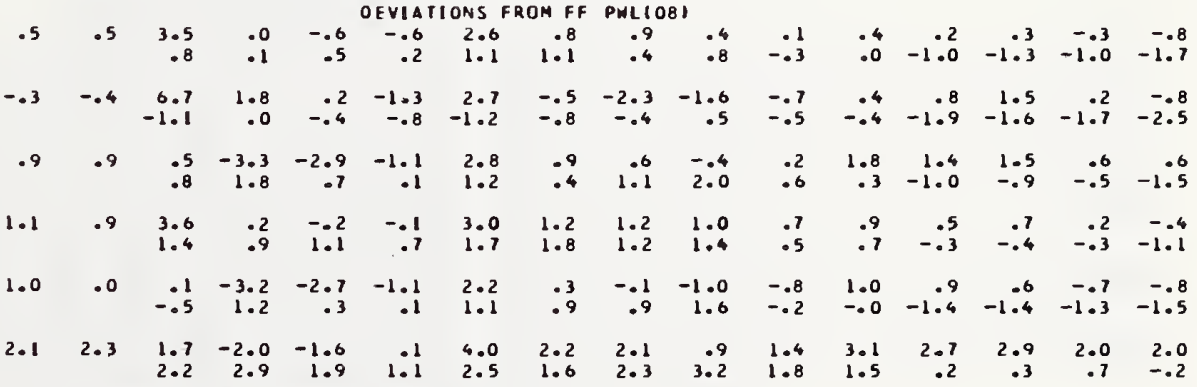
TABLE OF OCTAYE BANO POHER LEVELS

$\begin{array}{lllllllllll}4 & C & 31.5,63 & 125 & 250 & 500 & 1 K & 2 K & 4 K & 8 K\end{array}$

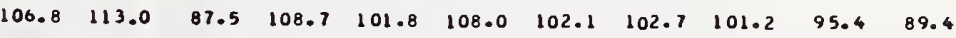

$\begin{array}{lllllllllll}107.8 & 113.4 & 0 & 110.4 & 103.0 & 107.5 & 102.8 & 101.8 & 101.6 & 97.4 & 92.7\end{array}$

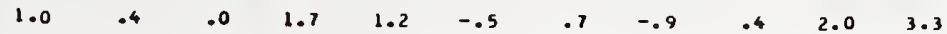




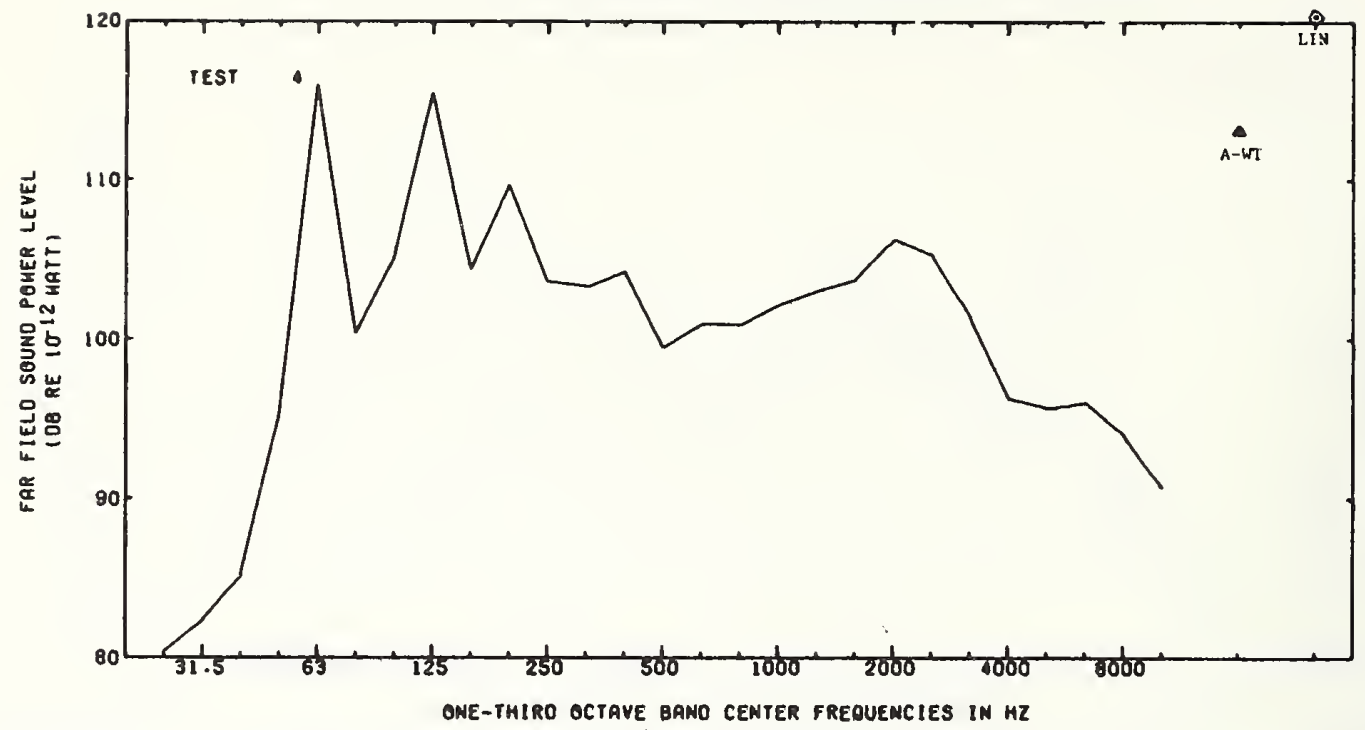

SIGNAL QUALITY

$\begin{array}{lllllllllllllllllllllllllllll}\text { FAR FIELO } & \mathbf{T} & \mathbf{F} & \mathbf{N} & \mathbf{T} & \mathbf{T} & \mathbf{T} & \mathbf{T} & \mathbf{T} & \mathbf{T} & \mathbf{T} & \mathbf{N} & \mathbf{N} & \mathbf{N} & \mathbf{N} & \mathbf{N} & \mathbf{F} & \mathbf{F} & \mathbf{F} & \mathbf{F} & \mathbf{F} & \mathbf{F} & \mathbf{F} & \mathbf{F} & \mathbf{F} & \mathbf{F} & \mathbf{F} & \mathbf{F}\end{array}$

IEST NUMBER \&

33 NEAR FIELO MIC POSITIONS.OF HHICH 33 USEO IN NFI

COMPRESSOR SURFACE AREA $=10,38$ SO, ME TRE S
CONFORMAL SURFACE AREA $(R=1 M)=34.73$ SO. METRES

WF IGHT ING-LOH FREOUENCY
HIGH FREOUENCY
FAR FIELD PWL
NF PHL 1
NF PWL 2
NF PWL 3
NF PWL 6
NF PHL 7
NF PWL 8

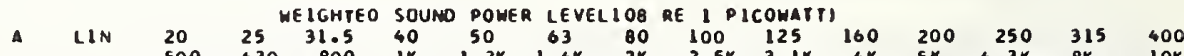

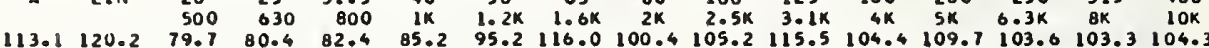
$99.5101 .0100 .9102 .2103 .1 \quad 103.8106 .3105 .3 \quad 101.696 .3 \quad 95.796 .194 .090 .7$

$114.3121 .2 \quad 84.0 \quad 83.5 \quad 85.2 \quad 86.0 \quad 96.8 \quad 117.1 \quad 100.4 \quad 106.7 \quad 116.2 \quad 105.3 \quad 110.8 \quad 103.9102 .9103 .3$ $\begin{array}{llllllllllllll}101.0 & 102.8 & 102.5 & 102.2 & 103.9 & 104.7 & 107.9 & 107.2 & 102.2 & 97.2 & 96.6 & 96.8 & 94.2 & 90.9\end{array}$

$113.8120 .9 \quad 83.6 \quad 83.1 \quad 84.4 \quad 84.7 \quad 94.9114 .7 \quad 98.3106 .3117 .6105 .8111 .7103 .6102 .9102 .6$ $99.7102 .1 \quad 101.8102 .5103 .7104 .1 \quad 107.0106 .1 \quad 103.198 .097 .297 .995 .091 .8$

$115.4123 .0 \quad 89.5 \quad 88.6 \quad 89.4 \quad 87.7 \quad 97.2 \quad 119.0103 .3109 .4118 .6108 .0111 .8107 .0103 .1106 .1$ $103.2106 .4102 .5103 .2105 .1 \quad 107.1109 .1 \quad 105.9102 .899 .697 .598 .795 .992 .8$

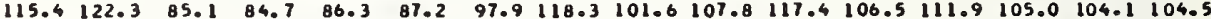
$102.2104 .0103 .7103 .4105 .0105 .9109 .0108 .4 \quad 103.498 .497 .798 .095 .492 .1$

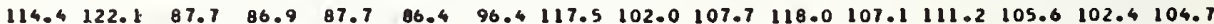
$102.2105 .1 \quad 102.0102 .5104 .3105 .9109 .0 \quad 106.3102 .199 .798 .098 .794 .992 .0$

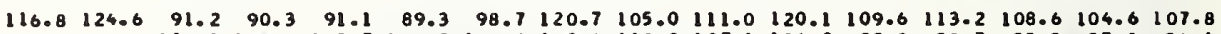
104.9108 .0103 .7104 .5106 .4108 .6110 .5107 .1104 .099 .998 .799 .997 .294 .1
\end{abstract}

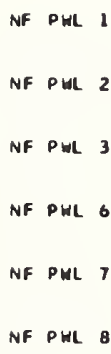

FAR FIELO PWL

FAR FIELD METHOOOLOGY PHL OEVIATIONS OF FF METH FROM FF

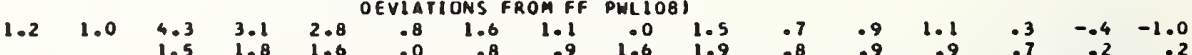

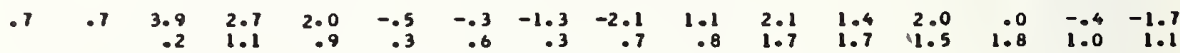

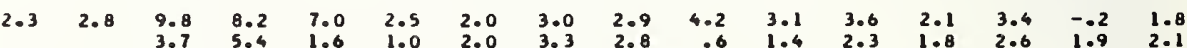

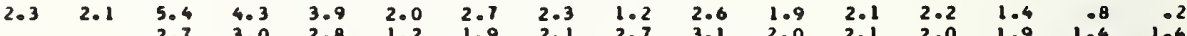

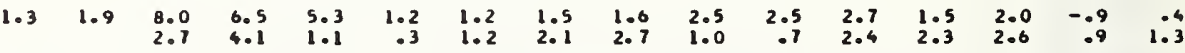

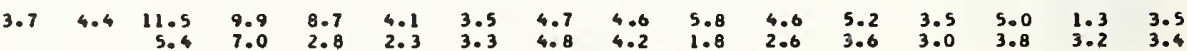

table of octaye bano power levels

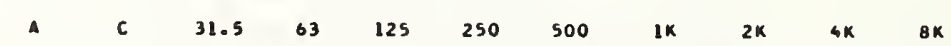

$\begin{array}{lllllllllll}113.1 & 120.4 & 87.9 & 116.2 & 116.2 & 111.4 & 106.8 & 106.9 & 110.0 & 103.4 & 98.9\end{array}$ $\begin{array}{lllllllllll}114.6 & 120.4 & .0 & 117.8 & 114.7 & 110.1 & 108.1 & 107.5 & 110.0 & 105.5 & 102.9\end{array}$

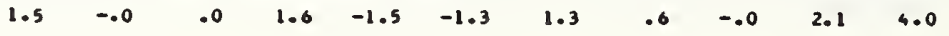




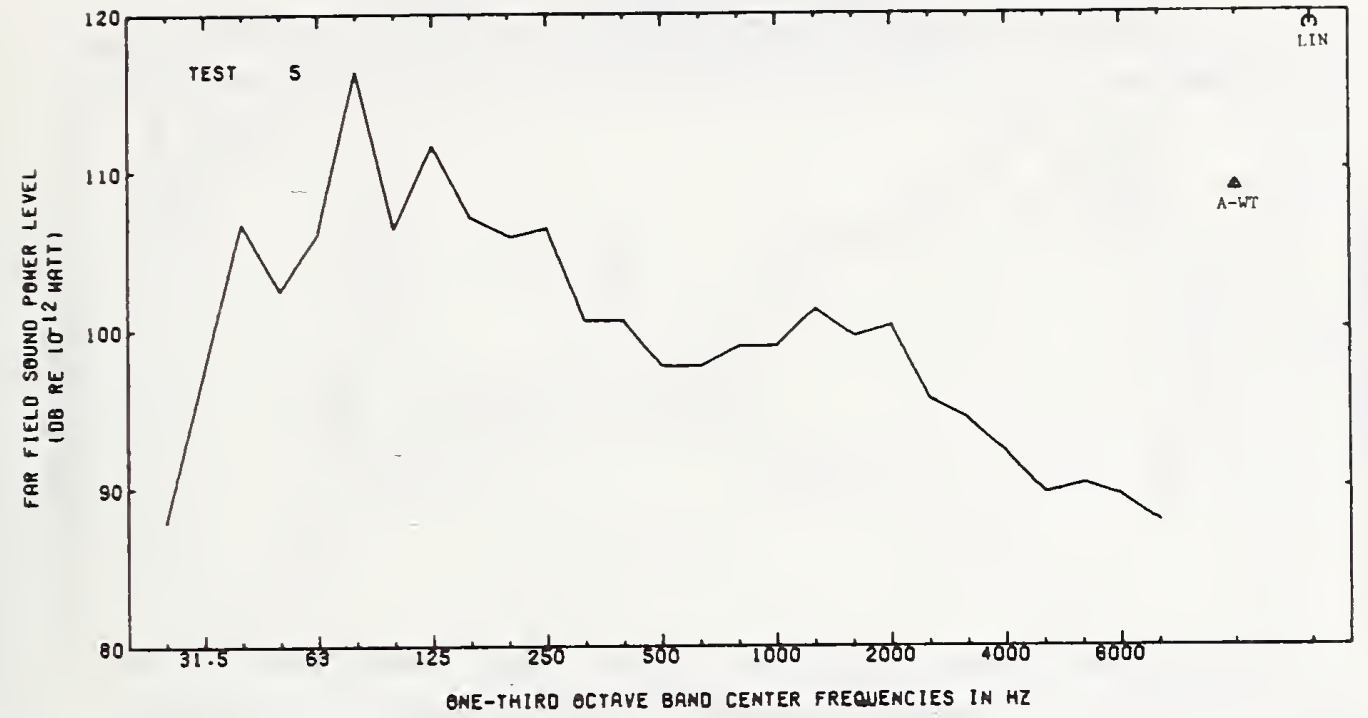

SIGNAL QUALITY

$\begin{array}{lllllllllllllllllllllllllllll}\text { FAR FIELD } & \text { N } & \text { T } & \text { T } & \text { T } & \text { T } & \text { T } & \text { T } & \text { T } & \text { T } & \text { N } & \text { T } & \text { N } & \text { N } & \text { N } & \text { N } & \text { N } & \text { N } & \text { F } & \text { F } & \text { F } & \text { F } & \text { F } & \text { F } & \text { F } & F & F & F \\ \text { NEAR FIELD } & \text { F } & \text { F } & \text { T } & \text { N } & \text { T } & \text { T } & \text { T } & \text { T } & \text { T } & \text { T } & \text { T } & \text { N } & \text { N } & \text { N } & \text { N } & \text { N } & \text { N } & \text { N } & \text { N } & \text { F } & \text { F } & \text { F } & \text { F } & \text { F } & \text { F } & \text { F } & \text { F }\end{array}$

TEST NUMBER 5

ST NFAR FIELO MIC POSITIONS, OF WHICH 33 USEO IN NFI

COMPRESSOR SURFACE AREA $=10.22$ S0. METRES
CONEORMAL SURFACE AREA $(R=L M)=34.76$ SO. METRES

WE IGHTING LOW FREOUENCY
WIGH FREOUENCY
FAR FIELO PWL
NF P 1
NF PWL 2
NF PWL 3
NF P WL 6
NF PWL 7
NF PWL 8

NF PWL 1
NF PM 2
NF PWL 3
NF PWL 6
NF PW 7
NF PWL 8

FAR FIELO PWL

FAR FIEL O METHOOOLOGY PHL OEVIATIONS OF FF METH FRCM FF

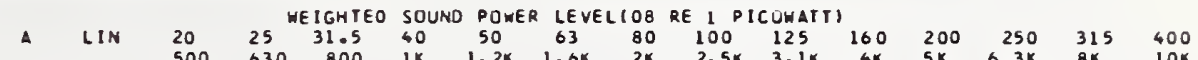

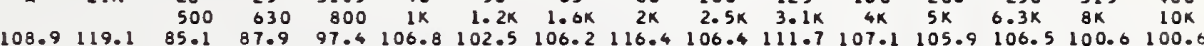

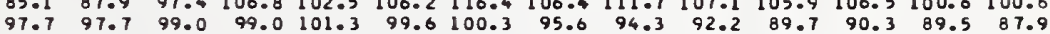

$109.6 \quad 119.8 \quad 99.6 \quad 100.1 \quad 101.4 \quad 110.1 \quad 101.5 \quad 110.9116 .1 \quad 107.1 \quad 111.1 \quad 109.2 \quad 105.7 \quad 106.5 \quad 100.5 \quad 98.8$ $\begin{array}{llllllllllllll}98.8 & 98.8 & 99.9 & 100.4 & 103.0 & 100.6 & 100.3 & 95.6 & 93.8 & 92.1 & 89.5 & 90.0 & 88.6 & 84.2\end{array}$

$\begin{array}{llllllllllllllll}107.5 & 117.6 & 81.1 & 90.8 & 96.0 & 107.1 & 97.5 & 109.6 & 114.3 & 103.2 & 104.3 & 106.6 & 103.1 & 105.5 & 98.0 & 97.5\end{array}$ $108.1 \quad 118.5 \quad 82.2 \quad 90.9 \quad 97.4 \quad 107.8 \quad 98.9 \quad 109.6 \quad 115.4 \quad 104.7 \quad 106.6 \quad 108.5 \quad 105.1 \quad 107.0 \quad 98.8 \quad 98.5$ $\begin{array}{lllllllllllllll}98.3 & 98.7 & 98.6 & 99.0 & 100.3 & 98.6 & 98.7 & 94.7 & 92.7 & 91.0 & 88.5 & 88.6 & 87.5 & 82.4\end{array}$

$109.9119 .9 \quad 98.4 \quad 99.1 \quad 100.8110 .1 \quad 101.2111 .3116 .3107 .1 \quad 110.7109 .0105 .7106 .8101 .0 \quad 99.1$ $\begin{array}{lllllllllllllll}99.1 & 99.1 & 100.4 & 100.6 & 103.6 & 101.1 & 100.7 & 95.9 & 94.2 & 92.4 & 89.7 & 90.3 & 88.8 & 84.4\end{array}$

$108.4118 .7 \quad 81.6 \quad 90.7 \quad 97.5107 .798 .8 \quad 109.7 \quad 115.7103 .9105 .5 \quad 107.6 \quad 105.4107 .498 .898 .5$

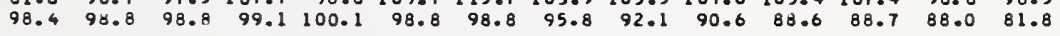

$\begin{array}{llllllllllllllll}109.3 & 119.7 & 83.4 & 92.1 & 98.7 & 108.9 & 100.2 & 110.8 & 116.7 & 106.0 & 107.9 & 109.6 & 106.4 & 108.1 & 99.9 & 99.7\end{array}$

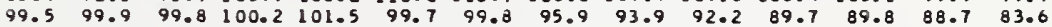

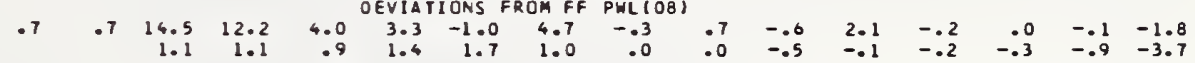

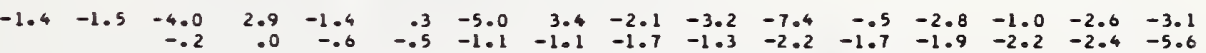

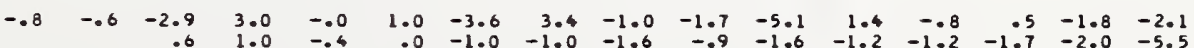

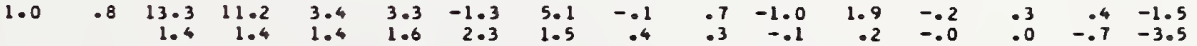

$$
\begin{aligned}
& \begin{array}{rrrrrrrrrrrrrrrr}
-.5 & -.4 & -3.5 & 2.8 & .1 & .9 & -3.7 & 3.5 & -.7 & -2.5 & -6.2 & .5 & -.5 & .9 & -1.8 & -2.1 \\
& & .7 & 1.1 & -.2 & .1 & -1.2 & -. .8 & -1.5 & .2 & -2.2 & -1.6 & -1.1 & -1.6 & -1.5 & -6.1
\end{array}
\end{aligned}
$$

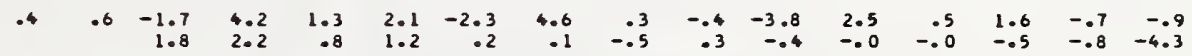

TABLE OF OCTAVE BANO POHER LEVELS

\begin{tabular}{rrrrrrrrrrr}
1 & \multicolumn{1}{c}{$C$} & 31.5 & 63 & 125 & 250 & 500 & $1 K$ & $2 K$ & $4 K$ & $8 K$ \\
108.9 & 119.3 & 107.3 & 117.0 & 113.9 & 109.8 & 103.7 & 104.7 & 103.7 & 97.2 & 94.1 \\
111.3 & 119.9 & .0 & 116.8 & 115.9 & 111.6 & 106.1 & 104.1 & 105.2 & 101.6 & 99.3 \\
2.4 & .6 & .0 & -.2 & 2.0 & 1.8 & 2.4 & -.6 & 1.5 & 4.4 & 5.2
\end{tabular}




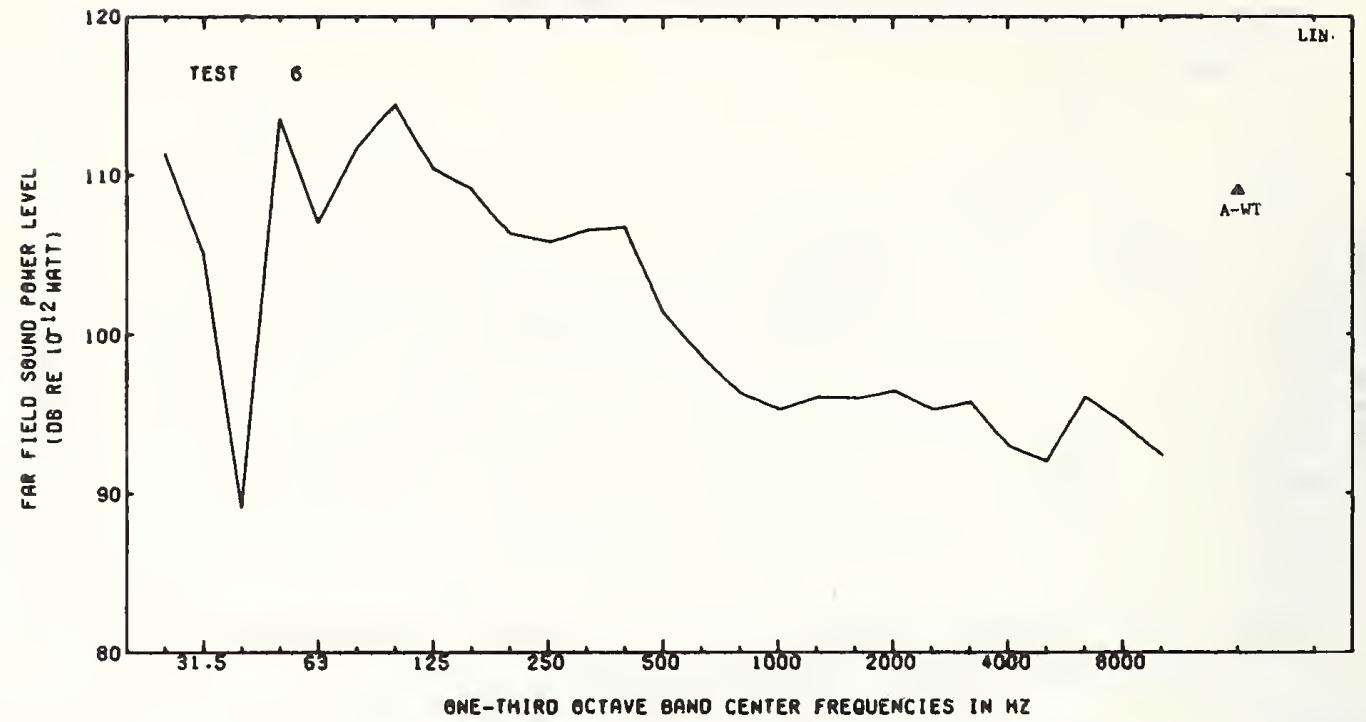

SIGNAL QUALITY $\begin{array}{lllllllllllllllllllllllllllllll}\text { FAR FIELD } & \mathbf{T} & \mathbf{T} & \mathbf{T} & \mathbf{T} & \mathbf{T} & \mathbf{T} & \mathbf{T} & \mathbf{T} & \mathbf{T} & \mathbf{T} & \mathbf{T} & \mathbf{T} & \mathbf{T} & \mathbf{N} & \mathbf{N} & \boldsymbol{F} & \mathbf{F} & \mathbf{F} & \boldsymbol{F} & \mathbf{F} & \mathbf{F} & \mathbf{F} & \mathbf{F} & \mathbf{F} & \boldsymbol{F} & \mathbf{F} & \boldsymbol{F} \\ \text { NELR FIELD } & \mathbf{T} & \mathbf{T} & \mathbf{T} & \mathbf{T} & \mathbf{T} & \mathbf{T} & \mathbf{T} & \mathbf{T} & \mathbf{T} & \mathbf{T} & \mathbf{T} & \mathbf{T} & \mathbf{T} & \mathbf{T} & \mathbf{N} & \mathbf{F} & \mathbf{F} & \mathbf{F} & \mathbf{F} & \mathbf{F} & \boldsymbol{F} & \mathbf{F} & \mathbf{F} & \mathbf{F} & \mathbf{F} & \boldsymbol{F} & \mathbf{F}\end{array}$ IFSI NUMATR O 33 NEAR FIELO YIC PUSITIUNS,OF WHICH 29 USED IN NFI CIMPRESSIIK SURFACE AHEA $=7.24$ SO. METKES

CONFORMAL SURFACE AREA $(R=1 M)=29.32$ SO. METKES
CONG

WU IGHTING-LOW FFFOUENCY
HIGH FKFQUENCY
FAR FIELO PWL
NF DWL 1
NF PWL 2
NF PWL 3
NF PWL 6
NF PWL 7
NF PWL 8

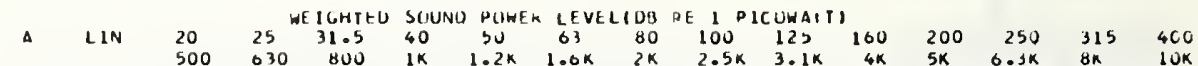

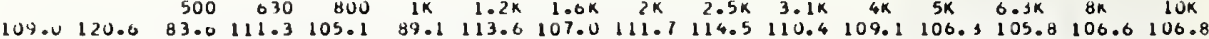
$101.4 \quad 48.696 .395 .390 .196 .090 .595 .395 .899 .092 .096 .194 .492 .4$ $109.3121 .0 \quad 83.7111 .1105 .8 \quad 89.1113 .5107 .4111 .3114 .7111 .4109 .3106 .9106 .9107 .0106 .7$

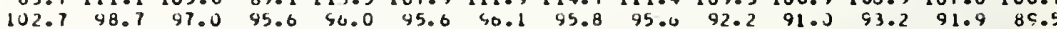
$108.4 \quad 119.5 \quad 82.7 \quad 111.1 \quad 105.4 \quad 89.0 \quad 113.4 \quad 107.5 \quad 110.3110 .2 \quad 108.4106 .0 \quad 105.4 \quad 105.0105 .8 \quad 105.9$

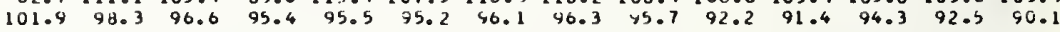

$109.0121 .2 \quad 80.8113 .2107 .7 \quad 90.4 \quad 115.5109 .9113 .5112 .1 \quad 110.1 \quad 107.3 \quad 107.0105 .8105 .8105 .6$

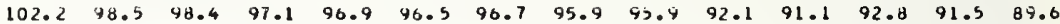

$110.4 \quad 122.0 \quad 84.9 \quad 112.4 \quad 107.0 \quad 90.5 \quad 114.8109 .1 \quad 113.1 \quad 115.5 \quad 112.2 \quad 110.4107 .9107 .9108 .0107 .8$ 103.899 .998 .296 .897 .290 .897 .397 .290 .493 .492 .494 .793 .390 .8

$103.9120 .6 \quad 85.6112 .3107 .2 \quad 89.9 \quad 114.4 \quad 109.5112 .5 \quad 112.4109 .8106 .6100 .4 \quad 106.0106 .7106 .4$

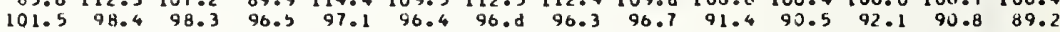

$110.3122 .6 \quad 88.4 \quad 114.7109 .3 \quad 91.9117 .0111 .5115 .2113 .3111 .4108 .7108 .4107 .1107 .0106 .8$ $103.459 .9 \quad 99.9 \quad 58.796 .598 .0 \quad 98.0 \quad 97.2 \quad 97.2 \quad 73.492 .394 .192 .890 .8$
\end{abstract}

\begin{tabular}{|c|c|c|c|c|c|c|c|c|c|c|c|c|c|c|c|c|c|c|}
\hline \multirow[b]{2}{*}{ Nt } & \multirow[b]{2}{*}{ PWL } & \multirow[b]{2}{*}{1} & \multicolumn{16}{|c|}{ OEVIATIUNS FHOM FF OWLIDBI } \\
\hline & & & .3 & .4 & 1. 3 & $\begin{array}{r}-.2 \\
.1\end{array}$ & .7 & $\begin{array}{r}.0 \\
.3\end{array}$ & $\begin{array}{l}-.1 \\
-.1\end{array}$ & $\begin{array}{r}.9 \\
-.4\end{array}$ & $\begin{array}{r}.2 \\
-.4\end{array}$ & $\begin{array}{l}.2 \\
.5\end{array}$ & $\begin{array}{l}1.3 \\
-.2\end{array}$ & $\begin{array}{r}.2 \\
-.8\end{array}$ & $\begin{array}{r}.00 \\
-1.0\end{array}$ & $\begin{array}{r}1.1 \\
-2.9\end{array}$ & -2.5 & $\begin{array}{r}-.1 \\
-2.9\end{array}$ \\
\hline NF & PWL & 2 & .0 .6 & -1.1 & $\begin{array}{r}-.4 \\
.5\end{array}$ & $\begin{array}{l}-.2 \\
-.1\end{array}$ & .3 & $\begin{array}{r}-.1 \\
.1\end{array}$ & $\begin{array}{l}-.2 \\
-.6\end{array}$ & $\begin{array}{r}.5 \\
-.8\end{array}$ & $\begin{array}{r}-1.4 \\
-.4\end{array}$ & $\begin{array}{r}-4.3 \\
1.0\end{array}$ & $\begin{array}{r}-2.0 \\
-.1\end{array}$ & $\begin{array}{r}-2.5 \\
-.8\end{array}$ & $\begin{array}{l}-.9 \\
-.6\end{array}$ & $\begin{array}{r}-.8 \\
-1.8\end{array}$ & $\begin{array}{r}-.8 \\
-1.9\end{array}$ & $\begin{array}{r}-.9 \\
-2.3\end{array}$ \\
\hline NF & PWL & 3 & -.0 & .6 & $\begin{array}{r}3.2 \\
.8\end{array}$ & $\begin{array}{l}1.9 \\
-.1\end{array}$ & $\begin{array}{l}2.6 \\
2.1\end{array}$ & $\begin{array}{l}1.3 \\
1.8\end{array}$ & $\begin{array}{r}1.9 \\
.8\end{array}$ & $\begin{array}{r}2.9 \\
.5\end{array}$ & $\begin{array}{r}1.8 \\
.2\end{array}$ & -2.4 & $\begin{array}{r}-.3 \\
.1\end{array}$ & $\begin{array}{r}-1.8 \\
-.9\end{array}$ & $\begin{array}{r}.7 \\
-.9\end{array}$ & $\begin{array}{r}-.0 \\
-3.3\end{array}$ & $\begin{array}{r}-.8 \\
-2.9\end{array}$ & $\begin{array}{l}-1.2 \\
-2.8\end{array}$ \\
\hline NF & PWL & 6 & 1.4 & 1.4 & $\begin{array}{l}1.3 \\
2.4\end{array}$ & $\begin{array}{l}1.1 \\
1.3\end{array}$ & $\begin{array}{l}1.9 \\
1.9\end{array}$ & $\begin{array}{l}1.4 \\
1.5\end{array}$ & $\begin{array}{l}1.2 \\
1.1\end{array}$ & $\begin{array}{r}2.1 \\
.8\end{array}$ & $\begin{array}{r}1.4 \\
.8\end{array}$ & $\begin{array}{l}1.0 \\
1.9\end{array}$ & $\begin{array}{l}1.8 \\
1.0\end{array}$ & $\begin{array}{r}1.3 \\
.4\end{array}$ & $\begin{array}{r}1.6 \\
.4\end{array}$ & $\begin{array}{r}2.1 \\
-1.4\end{array}$ & $\begin{array}{r}1.4 \\
-1.1\end{array}$ & $\begin{array}{r}1.0 \\
-1.0\end{array}$ \\
\hline NF & PWL & 7 & -.1 & -.0 & $\begin{array}{r}2.0 \\
.1\end{array}$ & $\begin{array}{l}1.0 \\
-.2\end{array}$ & $\begin{array}{l}2.1 \\
2.0\end{array}$ & 1.8 & 1.8 & $\begin{array}{r}2.5 \\
.4\end{array}$ & $\begin{array}{l}.8 \\
.3\end{array}$ & $\begin{array}{r}-2.1 \\
1.0\end{array}$ & $\begin{array}{r}-.6 \\
.9\end{array}$ & $\begin{array}{l}-2.5 \\
-1.6\end{array}$ & $\begin{array}{r}.1 \\
-1.5\end{array}$ & -4.2 & $\begin{array}{r}.1 \\
-3.6\end{array}$ & $\begin{array}{r}-.4 \\
-3.2\end{array}$ \\
\hline NF & PUL & 8 & 1.3 & 2.0 & $\begin{array}{l}4.8 \\
2.0\end{array}$ & $\begin{array}{l}3.4 \\
1.3\end{array}$ & $\begin{array}{l}4.2 \\
3.6\end{array}$ & $\begin{array}{l}2.8 \\
3.4\end{array}$ & $\begin{array}{l}3.4 \\
2.4\end{array}$ & $\begin{array}{l}4.5 \\
2.0\end{array}$ & $\begin{array}{l}3.5 \\
1.5\end{array}$ & $\begin{array}{r}-1.2 \\
1.9\end{array}$ & $\begin{array}{l}1.0 \\
1.4\end{array}$ & $\begin{array}{r}-.4 \\
.4\end{array}$ & $\begin{array}{r}2.1 \\
.3\end{array}$ & $\begin{array}{r}1.3 \\
-2.0\end{array}$ & -1.46 & $-1 \dot{0}$ \\
\hline
\end{tabular}
TABLE OF OCIAVE BANO POHER LEVELS

FAR FIELO PWL

FAR FIELO METHOOOL OGY PHL

DEVIATIONS OF FF METH FRUM FF

$\begin{array}{rrrrrrrrrrr}1 & C & 31.5 & 63 & 125 & 250 & 500 & 1 K & 2 K & 4 K & 8 K \\ 109.0 & 120.0 & 112.3 & 116.3 & 116.7 & 111.0 & 108.4 & 100.7 & 100.7 & 98.7 & 99.3 \\ 110.8 & 122.2 & 112.3 & 117.1 & 120.1 & 112.5 & 110.4 & 99.6 & 98.6 & 99.5 & 100.8 \\ 1.8 & 2.2 & .0 & .8 & 3.4 & 1.5 & 2.0 & -1.1 & -2.1 & .8 & 1.5\end{array}$




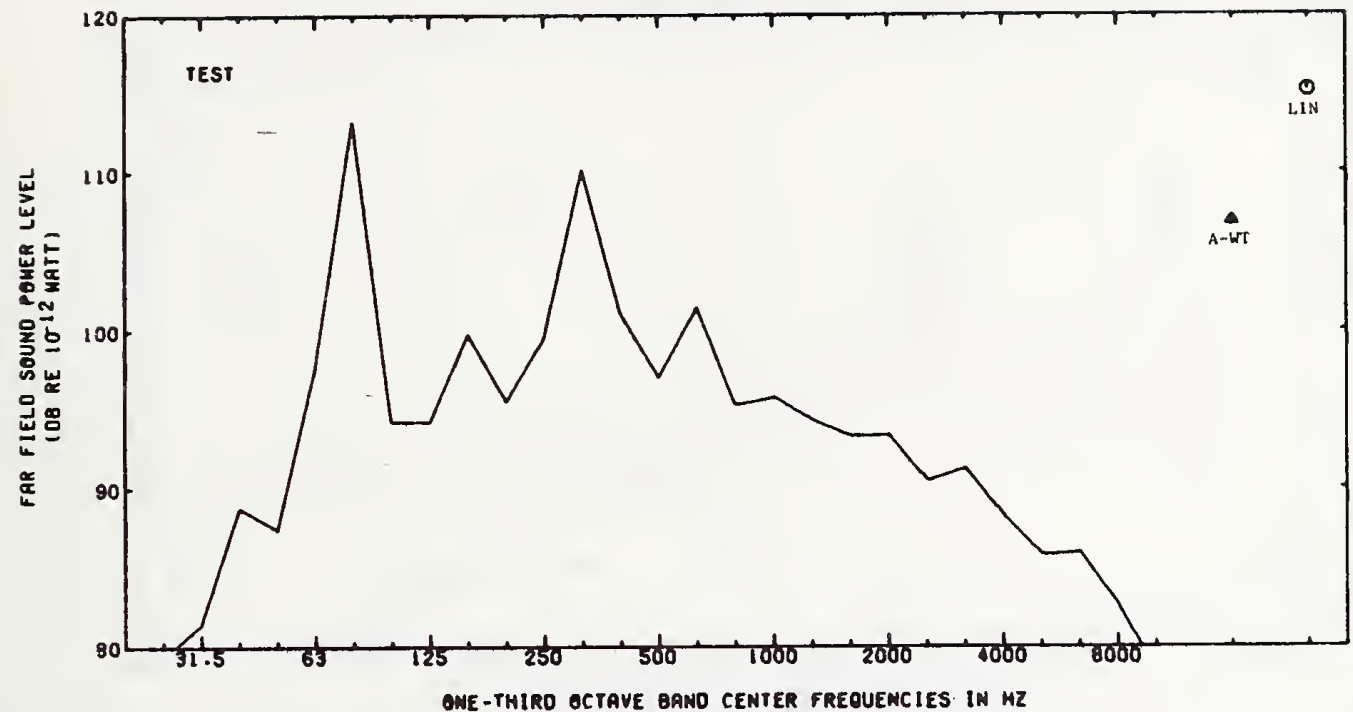

SIGNAL QUALITY

$\begin{array}{llllllllllllllllllllllllllllll}\text { FAR FIELD } & \mathbf{4} & \mathbf{N} & \mathbf{N} & \mathbf{N} & \mathbf{T} & \mathbf{T} & \mathbf{T} & \mathbf{N} & \mathbf{T} & \mathbf{N} & \mathbf{T} & \mathbf{T} & \mathbf{N} & \mathbf{N} & \mathbf{N} & \mathbf{N} & \mathbf{F} & \mathbf{F} & \mathbf{F} & \mathbf{F} & \mathbf{F} & \mathbf{F} & \mathbf{F} & \mathbf{F} & \mathbf{F} & \mathbf{F} & \mathbf{F} \\ \text { NEAR FIELD } & \mathbf{F} & \mathbf{F} & \mathbf{N} & \mathbf{N} & \mathbf{T} & \mathbf{T} & \mathbf{N} & \mathbf{N} & \mathbf{T} & \mathbf{N} & \mathbf{T} & \mathbf{T} & \mathbf{N} & \mathbf{N} & \mathbf{N} & \mathbf{F} & \mathbf{F} & \mathbf{F} & \mathbf{F} & \mathbf{F} & \mathbf{F} & \mathbf{F} & \mathbf{F} & \mathbf{F} & \mathbf{F} & \mathbf{F} & \mathbf{F}\end{array}$

TEST NUMAER 7

COMPRE SSOR SURFACE AREA=14.72 SO. METRES

ST NEAR FIELO MIC PUSITIONS, OF HHICH 45 USEO IN NFI

CONFORMAL SURFACE AREA $(R=1 M)=42 \cdot 81$ SO. METRES

WE IGHTING-LOW FRE OUENCY

FAR FIELD PUL FIG FREUENCY

NF PWL I

NF PWL 2

NF PUL 3

NF PUL 6

NF PWL 7

NF PWL 8

NF PUL 1

NF PWL 2

NF PHL 3

NF P WL 6

NF PUL 7

NF PUL 8

FAR FIELO PHL

FAR FIELO ME THOOOLOGY PML DEVIATIONS OF FF METH FROA FF

\begin{abstract}
$\begin{array}{llllllllllllllll}\text { A LIN IGHTEO SOUMD POHER LEVELIOB RE } & 1 \text { PICOHATII } \\ & 20 & 25 & 31.5 & 40 & 50 & 63 & 80 & 100 & 125 & 160 & 200 & 250 & 315 & 400 \\ & 500 & 630 & 800 & 1 K & 1.2 K & 1.6 K & 2 K & 2.5 K & 3.1 K & 4 K & 5 K & 6.3 K & 8 K & 10 K\end{array}$

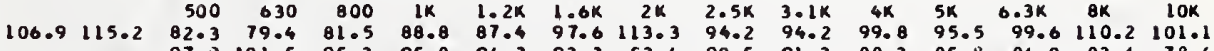

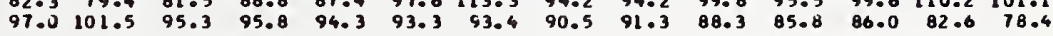

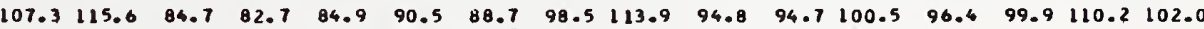
$\begin{array}{llllllllllllllll}98.2 & 102.2 & 96.1 & 95.9 & 94.7 & 94.1 & 93.8 & 90.9 & 91.5 & 88.4 & 85.5 & 86.5 & 82.8 & 78.8\end{array}$

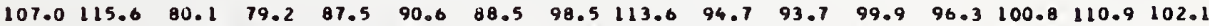

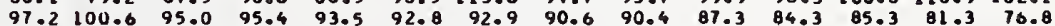

$109.0116 .8 \quad 02.6 \quad 79.5 \quad 82.0 \quad 90.7 \quad 89.1 \quad 97.5 \quad 114.0 \quad 94.7 \quad 94.4 \quad 101.3 \quad 97.3 \quad 102.0112 .5105 .5$

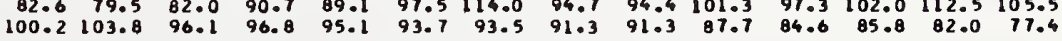

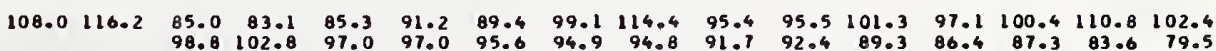

$108.7115 .5 \quad 81.8 \quad 79.5 \quad 01.6 \quad 90.5 \quad 88.8 \quad 96.7112 .6 \quad 93.6 \quad 93.4 \quad 100.8 \quad 96.6 \quad 101.1 \quad 111.5 \quad 104.3$ $\begin{array}{lllllllllllllll}99.8 & 102.8 & 96.8 & 96.8 & 94.1 & 92.9 & 92.7 & 90.8 & 90.8 & 88.3 & 83.7 & 86.2 & 81.5 & 77.7\end{array}$

$110.4 \quad 118.3 \quad 84.0 \quad 80.6 \quad 83.2 \quad 92.0 \quad 90.2 \quad 99.0 \quad 115.5 \quad 96.1 \quad 95.6 \quad 102.6 \quad 98.6 \quad 103.5 \quad 114.0 \quad 107.0$

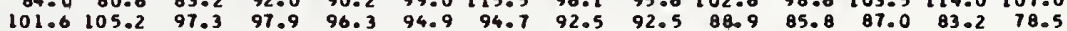

TABLE OF OCTAVE BANO POMER LEVELS

\begin{tabular}{rrrrrrrrrrr}
1 & \multicolumn{1}{c}{$C$} & 31.5 & 63 & 125 & 250 & \multicolumn{1}{c}{500} & $1 K$ & $2 K$ & $4 K$ & $8 K$ \\
106.9 & 115.7 & 89.9 & 113.4 & 101.7 & 110.7 & 105.1 & 99.9 & 97.4 & 93.8 & 80.1 \\
107.1 & 116.7 & 09.5 & 115.2 & 104.2 & 110.4 & 105.3 & 99.8 & 96.4 & 94.2 & 90.9 \\
.2 & 1.0 & -.4 & 1.8 & 2.5 & -.3 & .2 & -.1 & -1.0 & .4 & 2.8
\end{tabular}




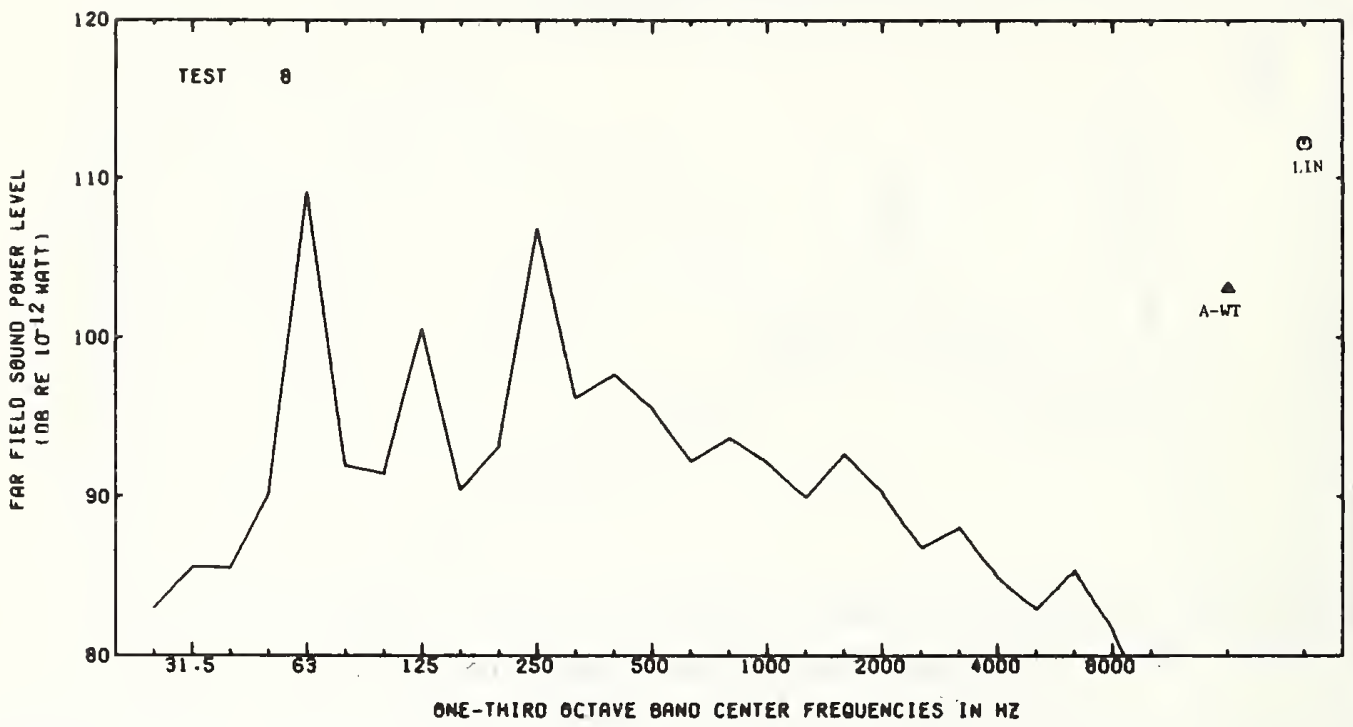

SIGNAL QUALITY

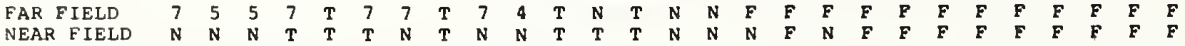

IEST NUMBER 6
COMPRESSOR SURFACE AREA 13.71 SO. METRES

CONFORMAL SURFACE AREA $(R=1 M)=41.29$ SO. METRES

WEIGHIING-LOW FRE OUENCY
HIGH FREOUENCY
FAR FIELO PHL
NF PHL 1
NF PWL 2
NF PUL 3
NF PHL 6
NF PUL 7
NF PHL 6

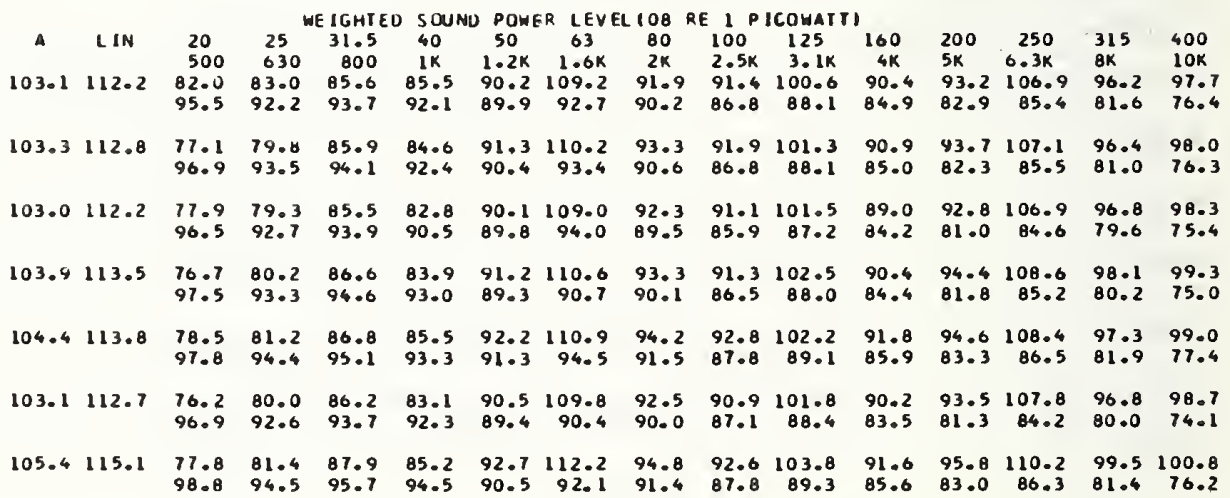

NF PWL 1

NF PHL 2

NF P WL 3

NF PWL 6

NF PWL 7

NF PHL 6

FAR FIELO PWL

far fielo methooologr pHL

OEVIATIONS OF FF METH FROM FF

OEVIATIONS FROM FF PHLIOBI

$\begin{array}{rrrrrrrrrrrrrrrr}.2 & .6 & -4.9 & -3.2 & .3 & -.9 & 1.1 & 1.0 & 1.4 & .5 & .7 & .5 & .5 & .2 & .2 & .3 \\ & & 1.4 & 1.3 & .4 & .3 & .5 & .7 & .4 & .0 & .0 & .1 & -.6 & .1 & -.06 & -. .1\end{array}$

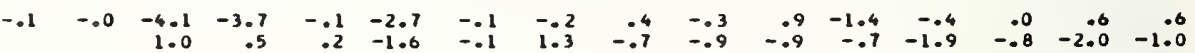

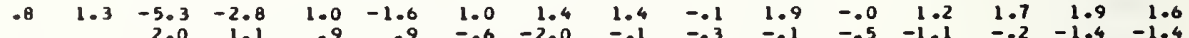

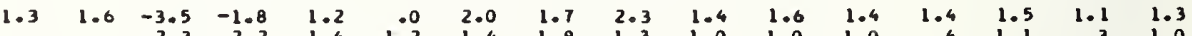

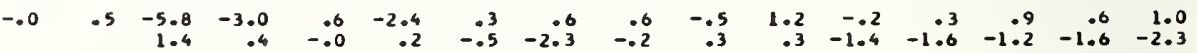

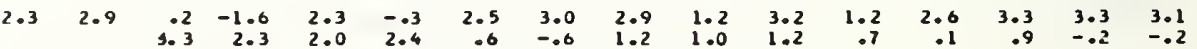

TABLE OF UCTAVE BANO POWER LEVELS

$\begin{array}{rrrrrrrrrrr}4 & C & 31.5 & 63 & 125 & 250 & 500 & 1 K & 2 K & 4 K & 8 K \\ 103.1 & 112.0 & 89.6 & 109.3 & 101.4 & 107.4 & 100.5 & 96.9 & 95.3 & 90.6 & 87.3 \\ 104.4 & 113.4 & .0 & 112.1 & 103.3 & 106.6 & 101.8 & 97.5 & 95.4 & 94.1 & 90.6 \\ 1.3 & 1.4 & .0 & 2.8 & 1.9 & -.8 & 1.3 & .6 & .1 & 3.5 & 3.3\end{array}$




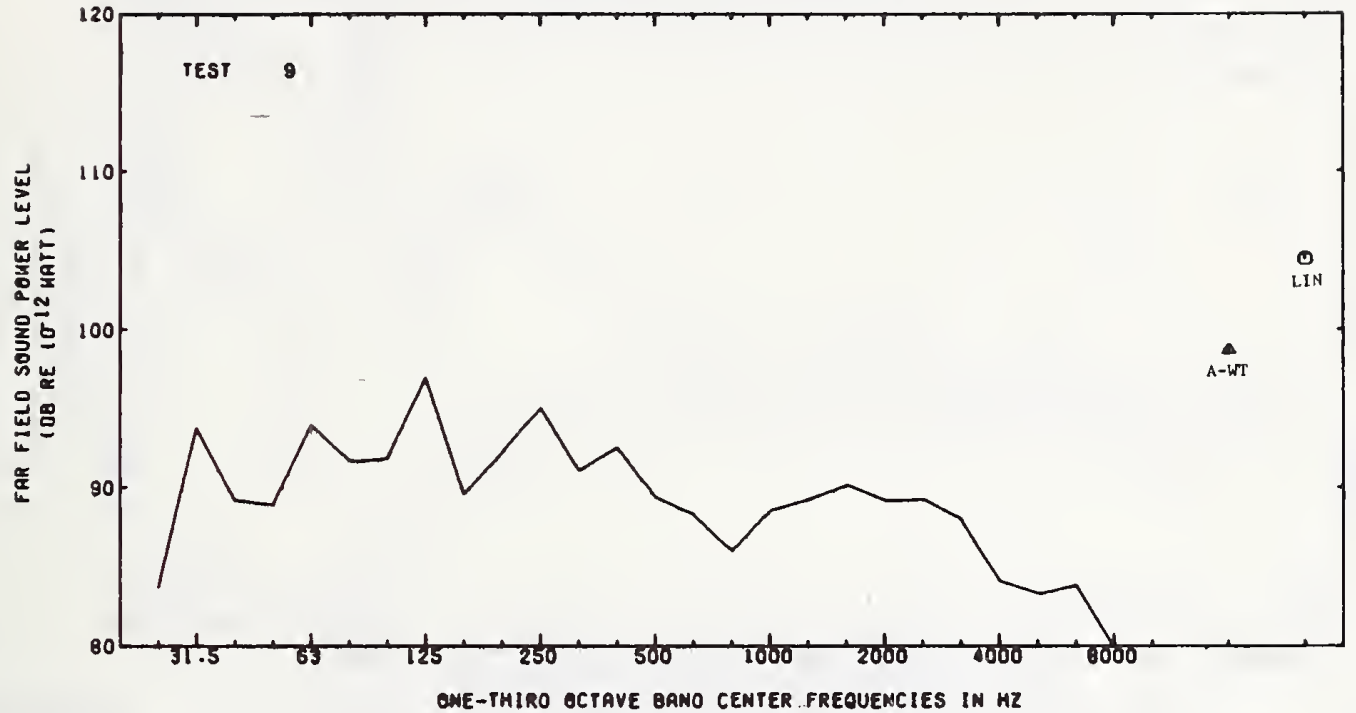

SIGNAL QUALITY

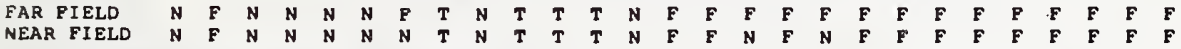

WE IGHIING-LOW FRE QUENCY
HIGH FREOUENCY
FAR FLFLO PHL
NF PML 1
NF PWL 2
NF DWL 3
NF PWL 6
NF PWL 7
NF PWL 8

NF PWL 1

NF PUL 2

NF P WL 3

NF PW 6

NF PUL 7

NF PWL 8

FAR FIELO PWL

far fielo me thoOOLOGY PHE DEVIATIONS OF FF METH FROM FF

\begin{abstract}
WE IGHTEU SUUNO POWER LEVELIOB RE 1 PICOWATTI

\begin{tabular}{|c|c|c|c|c|c|c|c|c|c|c|c|c|c|c|c|}
\hline $\begin{array}{l}4 \\
8.7\end{array}$ & $\begin{array}{c}\text { LIN } \\
104.5\end{array}$ & $\begin{array}{c}20 \\
500 \\
81.6 \\
89.4\end{array}$ & $\begin{array}{r}25 \\
630 \\
83.7 \\
88.3\end{array}$ & $\begin{array}{l}\text { E IGHT EO } \\
31.5 \\
800 \\
93.8 \\
86.0\end{array}$ & $\begin{array}{l}\text { SOUNO } \\
40 \\
1 \mathrm{~K} \\
89.2 \\
88.6\end{array}$ & $\begin{array}{l}\text { POWER } \\
\text { SO } \\
1.2 K \\
88.9 \\
89.3\end{array}$ & $\begin{array}{l}\text { LEV } \\
63 \\
1.6 K \\
9460 \\
90.2\end{array}$ & $\begin{array}{c}108 \\
80 \\
2 k \\
91.7 \\
89.2\end{array}$ & $\begin{array}{l}\text { E } 10 p \\
100 \\
2.5 k \\
91.9 \\
89.3\end{array}$ & $\begin{array}{c}\text { ICOWATT } \\
125 \\
3.1 K \\
97.0 \\
88.0\end{array}$ & $\begin{array}{l}160 \\
4 K \\
89.6 \\
84.1\end{array}$ & $\begin{array}{l}200 \\
5 K \\
92.3 \\
83.3\end{array}$ & $\begin{array}{r}250 \\
6.3 K \\
95.1 \\
83.9\end{array}$ & $\begin{array}{l}315 \\
8 K \\
91.1 \\
79.9\end{array}$ & $\begin{array}{l}400 \\
10 \mathrm{~K} \\
92.6 \\
78.6\end{array}$ \\
\hline 3.1 & 105.1 & $\begin{array}{l}82.0 \\
90.5\end{array}$ & $\begin{array}{l}84.8 \\
87.7\end{array}$ & $\begin{array}{l}95 . \\
87 .\end{array}$ & $\begin{array}{l}89.8 \\
88.2\end{array}$ & $\begin{array}{l}89.8 \\
88.4\end{array}$ & $\begin{array}{l}95.2 \\
89.3\end{array}$ & $\begin{array}{l}92.6 \\
88.5\end{array}$ & $\begin{array}{l}92.5 \\
87.3\end{array}$ & $\begin{array}{l}98.1 \\
85.8\end{array}$ & $\begin{array}{l}92.0 \\
82.4\end{array}$ & $\begin{array}{l}92.5 \\
80.5\end{array}$ & & & $\begin{array}{l}91.1 \\
75.1\end{array}$ \\
\hline .1 & 104.3 & $\begin{array}{l}81.6 \\
89.6\end{array}$ & $\begin{array}{l}84.0 \\
86.3\end{array}$ & $\begin{array}{l}95.6 \\
85.8\end{array}$ & $\begin{array}{l}88.8 \\
87.5\end{array}$ & $\begin{array}{l}89.1 \\
87.8\end{array}$ & $\begin{array}{l}96.1 \\
88.5\end{array}$ & $\begin{array}{l}92.0 \\
88.4\end{array}$ & $\begin{array}{l}91.4 \\
85.7\end{array}$ & $\begin{array}{l}97.7 \\
84.7\end{array}$ & $\begin{array}{l}92.5 \\
81.2\end{array}$ & & & & $\begin{array}{l}89.4 \\
75.3\end{array}$ \\
\hline 1.9 & 106.2 & $\begin{array}{l}82.4 \\
91.5\end{array}$ & $\begin{array}{l}5.5 \\
7.6\end{array}$ & $\begin{array}{l}99.2 \\
86.4\end{array}$ & $\begin{array}{l}90.1 \\
87.1\end{array}$ & $\begin{array}{l}90.0 \\
88.3\end{array}$ & $\begin{array}{l}96.0 \\
89.5\end{array}$ & $\begin{array}{l}92.3 \\
88.4\end{array}$ & $\begin{array}{l}93.0 \\
86.1\end{array}$ & $\begin{array}{r}101.1 \\
86.0\end{array}$ & $\begin{array}{l}93.3 \\
82.4\end{array}$ & & & & $\begin{array}{l}91.0 \\
76.3\end{array}$ \\
\hline . & 105.9 & $\begin{array}{l}83.4 \\
91.3\end{array}$ & $\begin{array}{l}85.6 \\
88.6\end{array}$ & $\begin{array}{l}96.1 \\
88.4\end{array}$ & $\begin{array}{l}90.7 \\
89.1\end{array}$ & $\begin{array}{l}90.6 \\
89.2\end{array}$ & $\begin{array}{l}96.0 \\
90.1\end{array}$ & $\begin{array}{l}93.4 \\
89.3\end{array}$ & $\begin{array}{l}93.3 \\
88.1\end{array}$ & & & & & & $\begin{array}{l}91.9 \\
76.0\end{array}$ \\
\hline 6.5 & 104. & $\begin{array}{l}82.0 \\
89.9\end{array}$ & $\begin{array}{l}34.5 \\
36.1\end{array}$ & $\begin{array}{l}97.2 \\
85.1\end{array}$ & $\begin{array}{l}88.7 \\
85.8\end{array}$ & $\begin{array}{l}88.6 \\
86.7\end{array}$ & $\begin{array}{l}94 \\
87\end{array}$ & $\begin{array}{l}91.9 \\
86.9\end{array}$ & $\begin{array}{l}92.1 \\
85.0\end{array}$ & $\begin{array}{r}100.0 \\
84.9\end{array}$ & & & & & $\begin{array}{l}89.4 \\
75.6\end{array}$ \\
\hline & & 12.4 & $\begin{array}{l}6.4 \\
8.5\end{array}$ & $\begin{array}{r}100.2 \\
87.3\end{array}$ & $\begin{array}{l}91.0 \\
88.0\end{array}$ & & & & $\begin{array}{l}94.0 \\
87.0\end{array}$ & $\begin{array}{r}102 \\
86\end{array}$ & $\begin{array}{l}94 \\
83\end{array}$ & & 81.8 & $\begin{array}{l}88.7 \\
79.0\end{array}$ & $\begin{array}{l}91.9 \\
77.2\end{array}$ \\
\hline
\end{tabular}
\end{abstract}

OEVIATIONS FROM FF PHL 1081

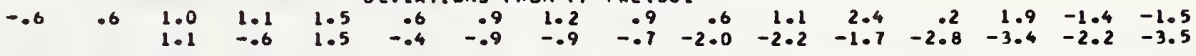

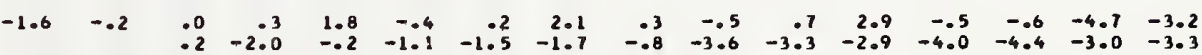

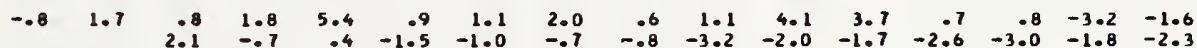

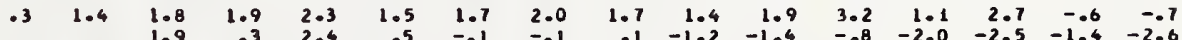
$\begin{array}{rlrrrrrrrrrrrrrr}-2.2 & .4 & .4 & .8 & 3.4 & -.5 & -. .3 & .6 & .2 & .2 & 3.0 & 3.0 & -.1 & -.6 & -4.7 & -3.2 \\ & .5 & -2.2 & -. .9 & -2.8 & -2.6 & -2.3 & -2.3 & -4.3 & -3.1 & -2.7 & -4.0 & -4.2 & -2.6 & -3.0\end{array}$

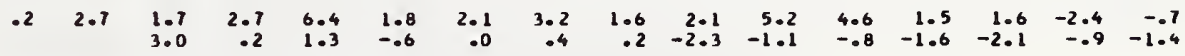
TABLE OF OCTAVE BANO POHER LEVELS

$\begin{array}{rrrrrrrrrrr}4 & C & 31.5 & 63 & 125 & 250 & 500 & 1 K & 2 K & 4 K & 8 K \\ 98.7 & 104.6 & 95.4 & 96.8 & 94.7 & 97.9 & 95.3 & 93.0 & 94.4 & 90.4 & 86.2 \\ 99.9 & 105.5 & 95.3 & 98.8 & 100.0 & 99.3 & 96.9 & 92.7 & 91.8 & 91.0 & 88.4 \\ 1.2 & .9 & -. .1 & 2.0 & 1.3 & 1.4 & 1.6 & -. .3 & -2.6 & .6 & 2.2\end{array}$




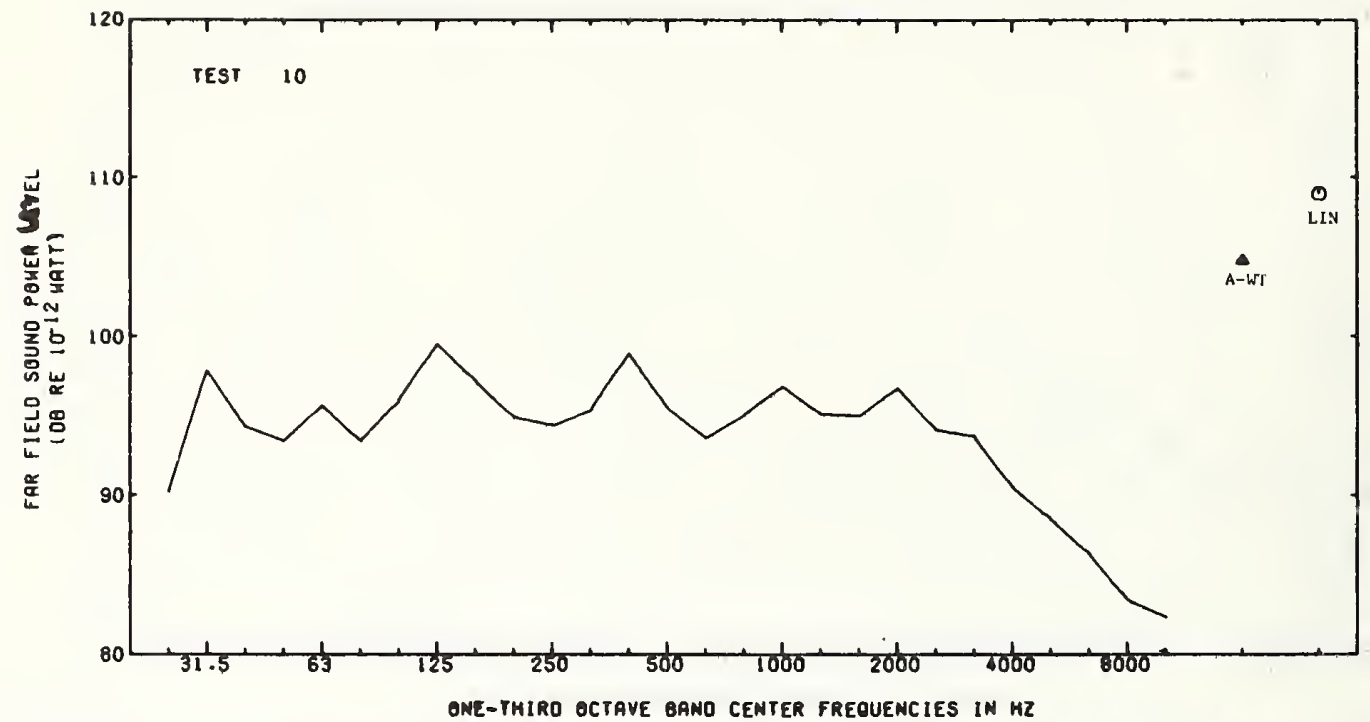

SIGNAL QUALITY

$\begin{array}{llllllllllllllllllllllllllllllll}\text { FAR FIELO } & \mathbf{4} & \mathbf{T} & \mathbf{N} & \mathbf{F} & \mathbf{N} & \mathbf{N} & \mathbf{N} & \mathbf{T} & \mathbf{N} & \mathbf{N} & \mathbf{N} & \mathbf{F} & \mathbf{N} & \mathbf{N} & \mathbf{F} & \mathbf{F} & \mathbf{F} & \mathbf{F} & \mathbf{F} & \mathbf{F} & \mathbf{F} & \mathbf{F} & \mathbf{F} & \mathbf{F} & \mathbf{F} & \mathbf{F} & \mathbf{F} \\ \text { NEAR FIELD } & \mathbf{F} & \mathbf{N} & \mathbf{N} & \mathbf{N} & \mathbf{N} & \mathbf{N} & \mathbf{N} & \mathbf{T} & \mathbf{N} & \mathbf{N} & \mathbf{N} & \mathbf{F} & \mathbf{N} & \mathbf{N} & \mathbf{F} & \mathbf{N} & \mathbf{N} & \mathbf{F} & \mathbf{F} & \mathbf{F} & \mathbf{F} & \mathbf{F} & \mathbf{F} & \mathbf{F} & \mathbf{F} & \mathbf{F} & \boldsymbol{F}\end{array}$

IEST NLMBER IO
COMPRESSOR SURFACE AREA $=41.92$ SO. METRES NEAR FIELO MIC POSITIONS.OF HHICH 83 USEO IN NFI

CONFORMAL SURFACE AREA $(R=1 M)=84.34$ SO. METRES

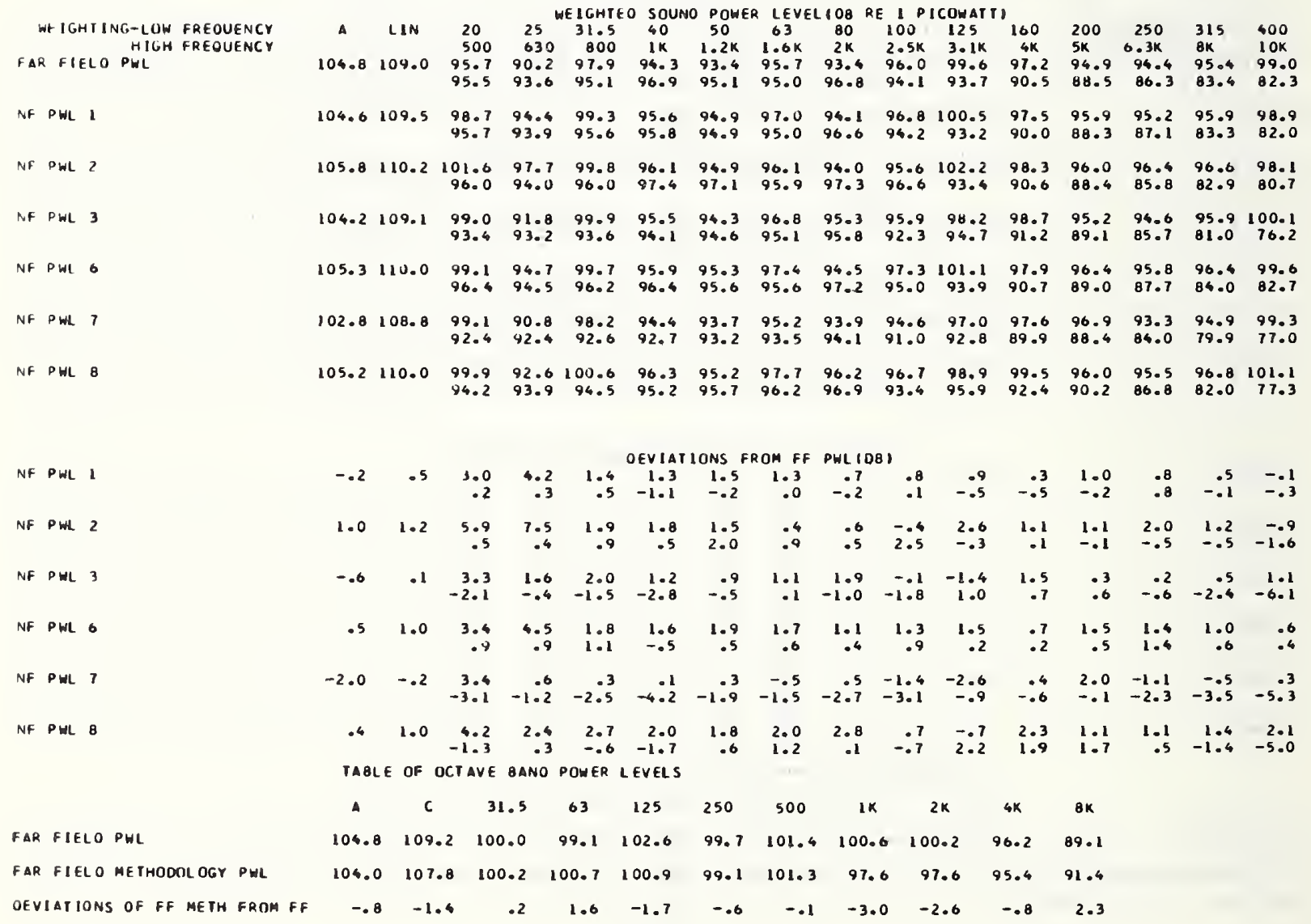




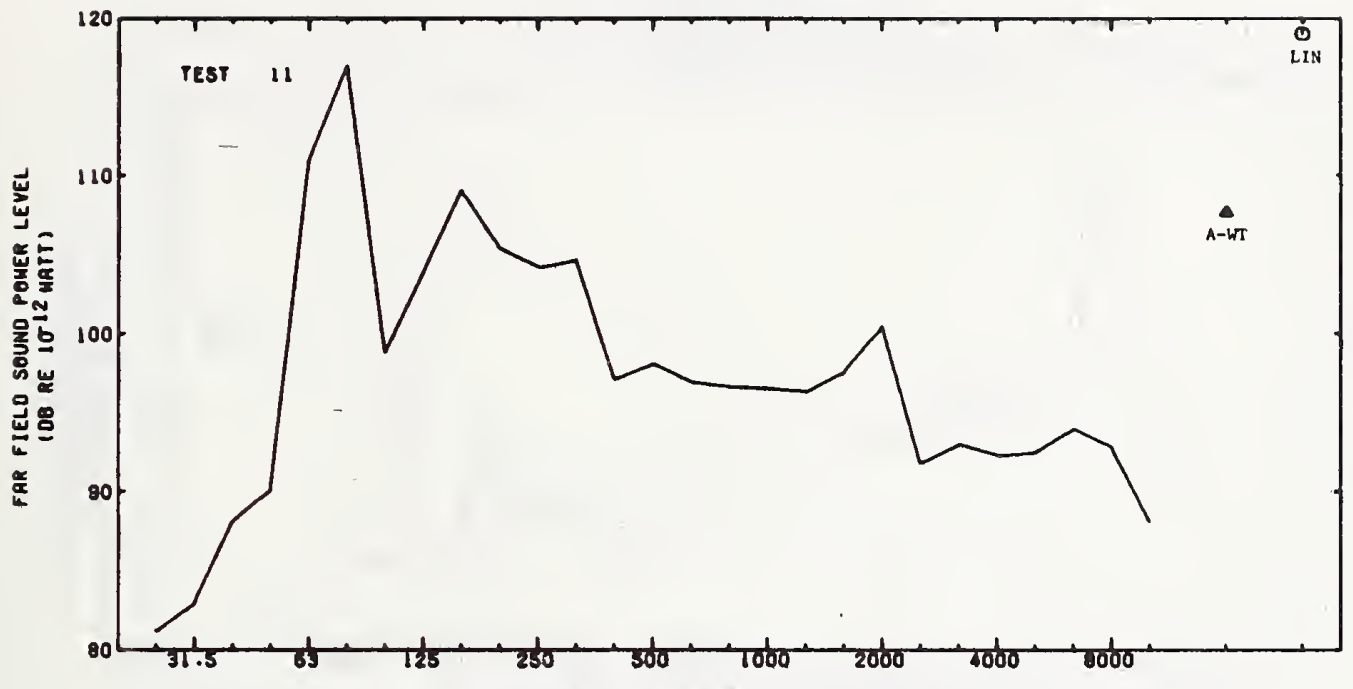

SIGNAL QUALITY

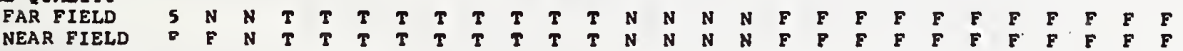
WF ICHTING-LOW FREQUENCY FAR FIELO PHL

NF PUL

NF PML 2

NF PM 3

NF PML 6

NF PHL 7

NF PHL 8

NF M 1

NF PWL 2

NF D 3

NF PUL 6

NF PUL 7

NF Pit $B$

FAR FIELO PUL

FAR FIELO WE TMODOLOGY PWL

DEVIATIONS OF FF METH FROM FF

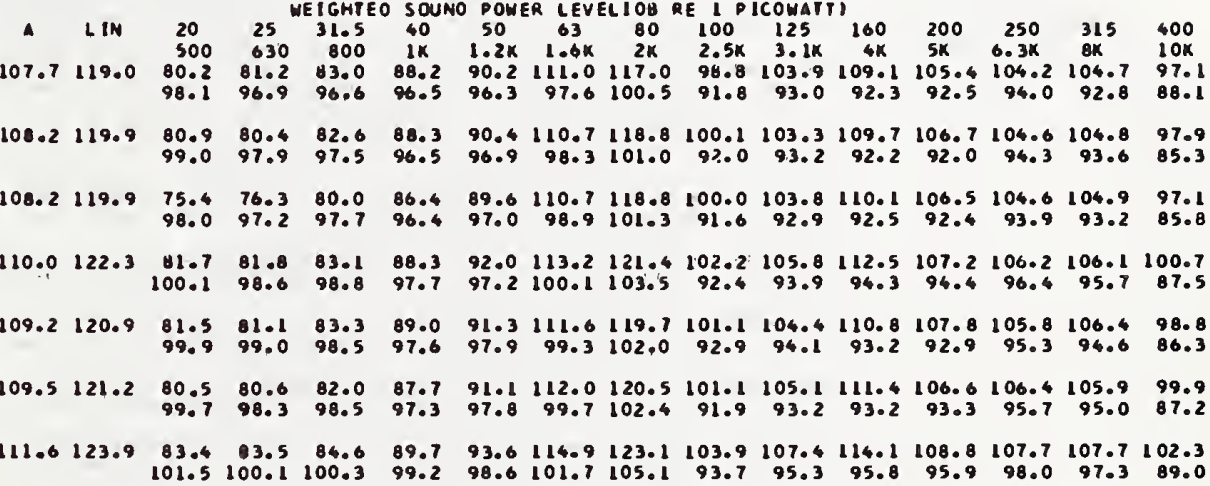
DEVIATIONS FROM FF PWL (OB)

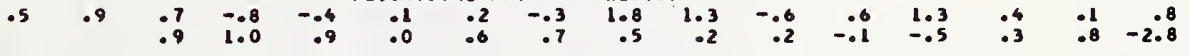

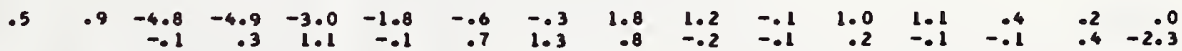

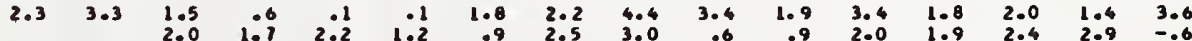

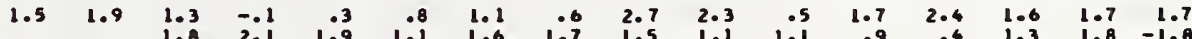

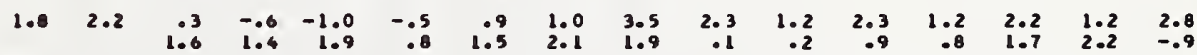

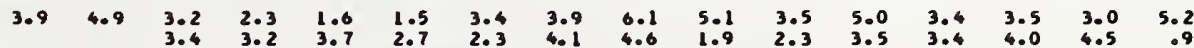
TABLE of octave Bano POMER leVels

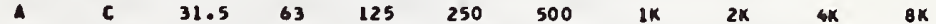

$\begin{array}{lllllllllll}107.7 & 110.9 & 90.0 & 118.0 & 110.5 & 109.6 & 102.2 & 101.2 & 102.7 & 97.4 & 97.0\end{array}$

$\begin{array}{lllllllllll}109.2 & 120.0 & .0 & 119.5 & 111.1 & 107.9 & 102.4 & 101.4 & 102.7 & 101.6 & 101.9\end{array}$

$\begin{array}{lllllllllll}1.5 & 1.1 & .0 & 1.5 & .6 & -1.7 & .2 & .2 & .0 & 4.2 & 4.9\end{array}$ 


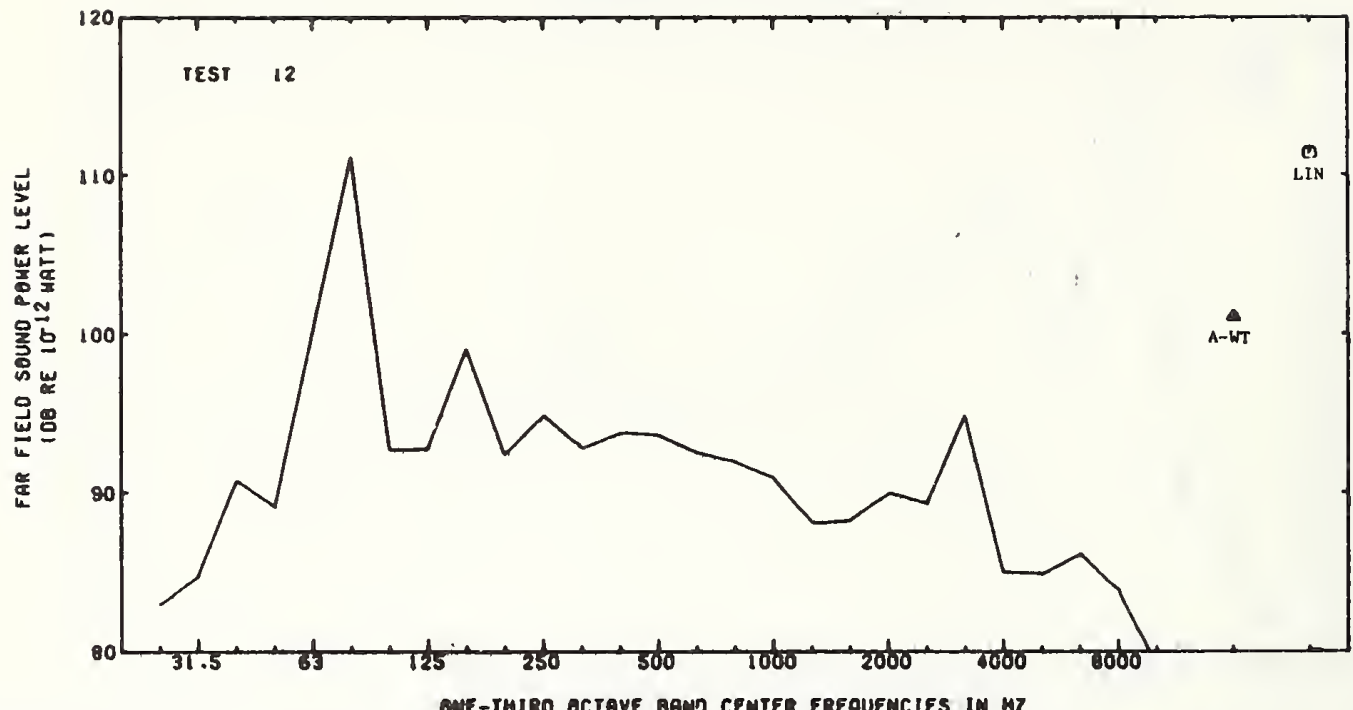

SIGNAL QUALITY

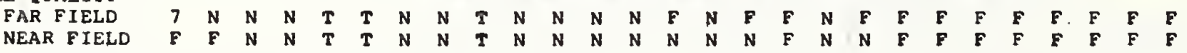

TEST NUMBER 12

COMPRESSOR SURFACE AREA 14.85 SO. METRES

47 NEAR FIELD MIIC PUSITIONS, OF HICH 43 USEO IN MFI

CONFORMAL SURFACE AREA $(R=1 \mathrm{M})=42.63$ SO. METRES

WFIGHTING-LOH FRE OUENCY
HIGH FREOUENCY
FAR FIELD PHL
NF PHL 1
NF PHL 2
NF PHL 3
NF PHL 6
NF PHL 7
NF PHL 8

NF PUL 1
NF PUL 2
NF PUL 3
NF PM 6
NF PUL 7
NF PUL 9

far fielo pHL

FAR FIELO METHODOLOGY PUL

OEVIATIONS OF FF METH FROM FF

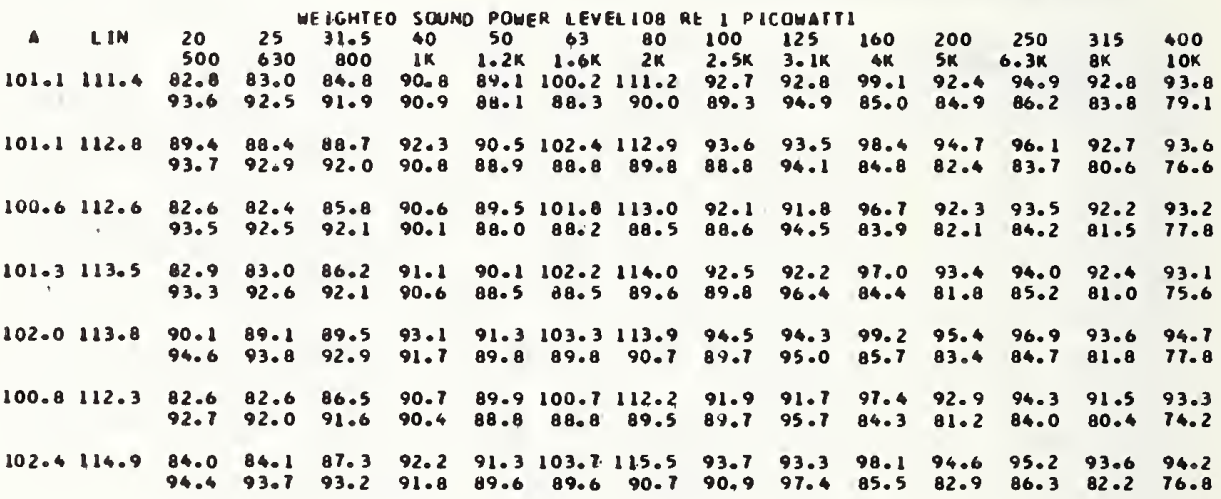

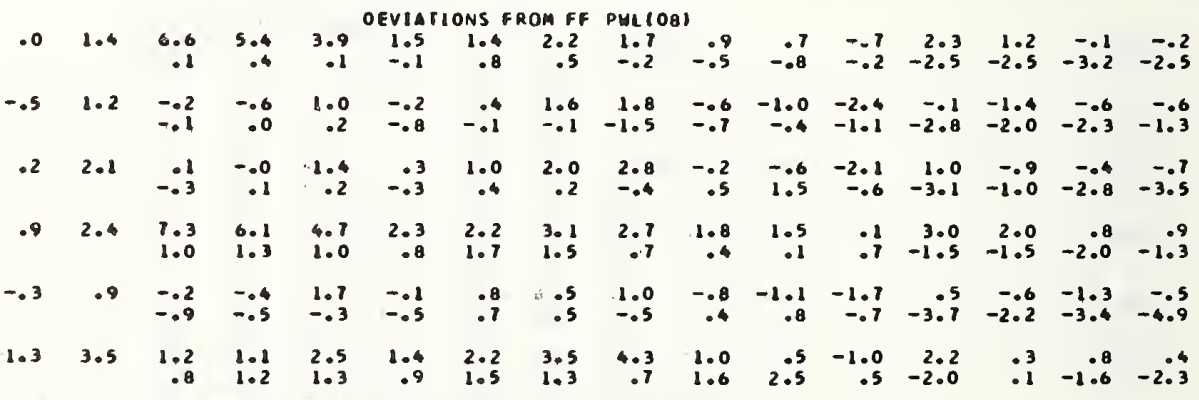
TABLE OF OCTAVE GANO POHER LEVELS

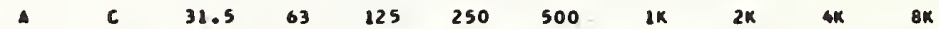

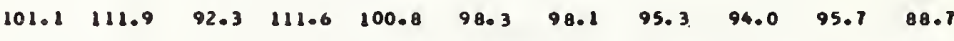

$103.7 \quad 115.0 \quad 92.2 \quad 115.0 \quad 102.2 \quad 98.7 .99 .7 \quad 94.4 \quad 95.4 \quad 97.8=92.4$

$\begin{array}{lllllllllllllllll}2.0 & 3.1 & -.1 & 3.4 & 1.4 & .4 & 1.6 & -.9 & 1.4 & 2.1 & .4 .7\end{array}$ 
Table 5.13

SOUND POWER LEVEL. DATA

Test Number 1

Compressor Output: $175 \mathrm{cfm}$

Englne/Compressor Type: Rotary ecrew, Diesel, Quieted

Compressor S1ze: $2.70 \times 1.29 \times 1.43$ m

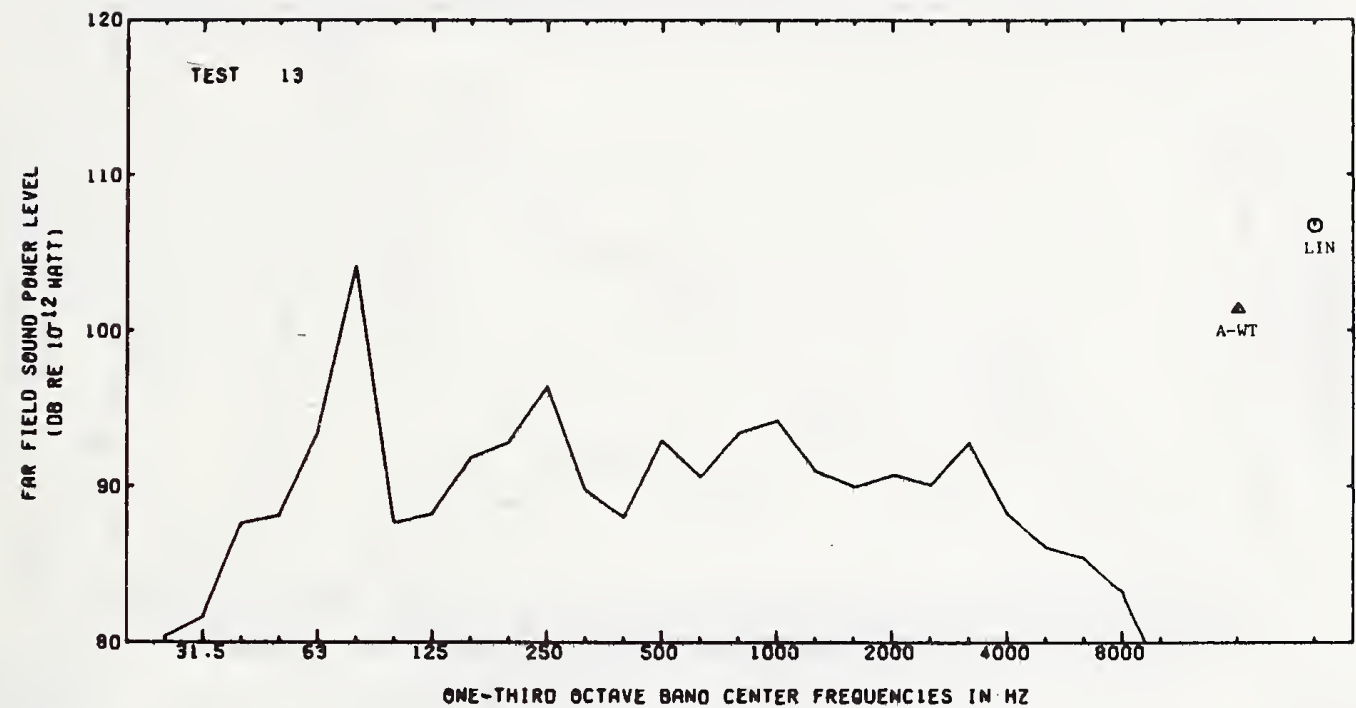

SIGNAL QUALITY

$\begin{array}{lllllllllllllllllllllllllllll}\text { FAR FIELD } & \mathbf{T} & \mathbf{F} & \mathrm{N} & \mathrm{N} & \mathbf{T} & \mathbf{T} & \mathbf{N} & \mathbf{N} & \mathbf{T} & \mathbf{T} & \mathbf{T} & \mathbf{T} & \mathbf{N} & \mathbf{N} & \mathbf{N} & \mathbf{F} & \mathbf{F} & \mathbf{N} & \mathbf{F} & \mathbf{F} & \mathbf{F} & \mathbf{F} & \mathbf{F} & \mathbf{F} & \mathbf{F} & \mathbf{F} & \mathbf{F} \\ \text { NEAR FIELO } & \mathbf{F} & \mathbf{F} & \mathbf{N} & \mathbf{N} & \mathbf{T} & \mathbf{T} & \mathbf{N} & \mathbf{N} & \mathbf{T} & \mathbf{T} & \mathbf{T} & \mathbf{T} & \mathbf{N} & \mathrm{N} & \mathbf{N} & \mathbf{F} & \mathbf{F} & \mathbf{N} & \mathbf{N} & \mathbf{F} & \mathbf{F} & \mathbf{F} & \mathbf{F} & \mathbf{F} & \mathbf{F} & \mathbf{F} & \mathbf{F}\end{array}$

TEST NUMBER 13 47 NEAR FIELD MIC POSITIONS, OF WHICH 43 USED IN NFI

COMPRE SSOR SURFACE AREA $=14.85$ SU. ME IRFS
CONFORMAL SURFACE AREA $(R=1 M)=42.63$ SO. METRES

\begin{tabular}{|c|c|c|c|c|c|c|c|c|c|c|c|c|c|c|c|c|c|c|c|c|}
\hline$F_{A P}$ & $\begin{array}{l}\text { WE IGr } \\
\text { FIE }\end{array}$ & & $\begin{array}{c}\text { NG- L OW } \\
\text { H IGH } \\
\text { PWL }\end{array}$ & $\begin{array}{l}\text { FREDUENCY } \\
\text { FRE OUENCY }\end{array}$ & 101.4 & $\begin{array}{c}\text { LIN } \\
100.8\end{array}$ & $\begin{array}{r}20 \\
500 \\
80.4 \\
93.0\end{array}$ & $\begin{array}{l}25 \\
630 \\
80.4 \\
90.6\end{array}$ & $\begin{array}{c}\text { E IGHT EO } \\
31.5 \\
800 \\
81.7 \\
93.5\end{array}$ & $\begin{array}{l}0 \text { SOUNO } \\
40 \\
1 K \\
87.7 \\
94.3\end{array}$ & $\begin{array}{l}\text { D POWER } \\
50 \\
1.2 K \\
88.2 \\
91.0\end{array}$ & $\begin{array}{l}\text { LFVE } \\
63 \\
1.6 \mathrm{~K} \\
93.5 \\
40.0\end{array}$ & $\begin{array}{c}108 \\
80 \\
2 \mathrm{~K} \\
104.2 \\
90.8\end{array}$ & $\begin{array}{l}\text { RE I P I } \\
100 \\
2.5 K \\
87.7 \\
90.1\end{array}$ & $\begin{array}{l}\text { CDWAT T } \\
125 \\
3.1 K \\
88.3 \\
92.9\end{array}$ & $\begin{array}{c}160 \\
4 K \\
91.9 \\
88.3\end{array}$ & $\begin{array}{l}200 \\
5 K \\
92.9 \\
86.1\end{array}$ & $\begin{array}{r}250 \\
6.3 \mathrm{~K} \\
96.5 \\
85.4\end{array}$ & $\begin{array}{l}315 \\
8 K \\
89.8 \\
83.2\end{array}$ & $\begin{array}{l}400 \\
10 K \\
88.0 \\
77.9\end{array}$ \\
\hline NF & PWL & 1 & - & & 101.1 & 107.2 & $\begin{array}{l}80.0 \\
93.4\end{array}$ & $\begin{array}{l}80.5 \\
90.7\end{array}$ & $\begin{array}{l}82.5 \\
93.7\end{array}$ & $\begin{array}{l}89.0 \\
94.1\end{array}$ & $\begin{array}{l}88.7 \\
91.1\end{array}$ & $\begin{array}{l}94.1 \\
90.4\end{array}$ & $\begin{array}{r}104.7 \\
90.8\end{array}$ & $\begin{array}{l}88.4 \\
88.8\end{array}$ & $\begin{array}{l}89.1 \\
90.3\end{array}$ & $\begin{array}{l}92.9 \\
87.0\end{array}$ & $\begin{array}{l}94.2 \\
83.0\end{array}$ & $\begin{array}{l}97.4 \\
82.7\end{array}$ & $\begin{array}{l}90.3 \\
80.0\end{array}$ & $\begin{array}{l}88.3 \\
74.4\end{array}$ \\
\hline NF & PWL & 2 & & & 99.1 & 105.5 & $\begin{array}{l}77.9 \\
90.5\end{array}$ & $\begin{array}{l}77.0 \\
88.9\end{array}$ & $\begin{array}{l}81.4 \\
93.4\end{array}$ & $\begin{array}{l}48.8 \\
92.0\end{array}$ & $\begin{array}{l}88.7 \\
89.2\end{array}$ & $\begin{array}{l}93.1 \\
88.7\end{array}$ & $\begin{array}{r}103.5 \\
88.4\end{array}$ & $\begin{array}{l}25.6 \\
85.9\end{array}$ & $\begin{array}{l}86.8 \\
88.5\end{array}$ & $\begin{array}{l}93.1 \\
85.2\end{array}$ & $\begin{array}{l}89.5 \\
80.9\end{array}$ & $\begin{array}{l}93.8 \\
81.2\end{array}$ & $\begin{array}{l}87.9 \\
78.4\end{array}$ & $\begin{array}{l}87.0 \\
72.9\end{array}$ \\
\hline NF & PHL & 3 & & & 99.2 & 105.6 & $\begin{array}{l}79.2 \\
90.1\end{array}$ & $\begin{array}{l}79.2 \\
89.1\end{array}$ & $\begin{array}{l}83.0 \\
93.1\end{array}$ & $\begin{array}{l}90.0 \\
92.0\end{array}$ & $\begin{array}{l}89.7 \\
89.3\end{array}$ & $\begin{array}{l}92.7 \\
88.9\end{array}$ & $\begin{array}{r}103.0 \\
88.4\end{array}$ & $\begin{array}{l}86.8 \\
86.2\end{array}$ & $\begin{array}{l}88.1 \\
88.4\end{array}$ & $\begin{array}{l}94.1 \\
85.0\end{array}$ & $\begin{array}{l}91.8 \\
80.8\end{array}$ & $\begin{array}{l}95.0 \\
81.1\end{array}$ & $\begin{array}{l}88.6 \\
78.0\end{array}$ & $\begin{array}{l}88.0 \\
72.5\end{array}$ \\
\hline NF & PWL & 6 & & & 102.0 & 108.1 & $\begin{array}{l}81.0 \\
94.4\end{array}$ & $\begin{array}{l}81.3 \\
91.6\end{array}$ & $\begin{array}{l}83.5 \\
94.6\end{array}$ & $\begin{array}{l}84.9 \\
94.9\end{array}$ & $\begin{array}{l}89.6 \\
92.0\end{array}$ & $\begin{array}{l}95.1 \\
91.3\end{array}$ & $\begin{array}{r}105.7 \\
91.7\end{array}$ & $\begin{array}{l}89.2 \\
89.7\end{array}$ & $\begin{array}{l}89.9 \\
91.3\end{array}$ & $\begin{array}{l}93.7 \\
88.0\end{array}$ & $\begin{array}{l}94.9 \\
84.0\end{array}$ & $\begin{array}{l}98.2 \\
83.7\end{array}$ & $\begin{array}{l}91.1 \\
81.0\end{array}$ & $\begin{array}{l}89.2 \\
75.4\end{array}$ \\
\hline NF & $P=L$ & 7 & & & 99.4 & 105.6 & $\begin{array}{l}79.3 \\
90.4\end{array}$ & $\begin{array}{l}78.9 \\
89.3\end{array}$ & $\begin{array}{l}81.9 \\
93.5\end{array}$ & $\begin{array}{l}89.6 \\
92.8\end{array}$ & $\begin{array}{l}89.4 \\
89.5\end{array}$ & $\begin{array}{l}92.5 \\
89.4\end{array}$ & $\begin{array}{r}102.7 \\
88.9\end{array}$ & $\begin{array}{l}87.3 \\
87.4\end{array}$ & $\begin{array}{l}88.2 \\
88.9\end{array}$ & $\begin{array}{l}93.1 \\
86.0\end{array}$ & $\begin{array}{l}91.1 \\
80.9\end{array}$ & $\begin{array}{l}93.9 \\
80.9\end{array}$ & $\begin{array}{l}88.5 \\
78.6\end{array}$ & $\begin{array}{l}88.1 \\
72.0\end{array}$ \\
\hline NF & $P G L$ & 8 & & & $100 \cdot 3$ & 106.8 & $\begin{array}{l}80.5 \\
91.2\end{array}$ & $\begin{array}{l}80.5 \\
90.2\end{array}$ & $\begin{array}{l}84.3 \\
94.2\end{array}$ & $\begin{array}{l}91.2 \\
93.0\end{array}$ & $\begin{array}{l}90.9 \\
90.4\end{array}$ & $\begin{array}{l}93.9 \\
90.1\end{array}$ & $\begin{array}{r}104.2 \\
89.6\end{array}$ & $\begin{array}{l}88.0 \\
87.3\end{array}$ & $\begin{array}{l}89.2 \\
89.6\end{array}$ & $\begin{array}{l}95.1 \\
86.2\end{array}$ & $\begin{array}{l}93.0 \\
82.0\end{array}$ & $\begin{array}{l}96.1 \\
82.2\end{array}$ & $\begin{array}{l}89.8 \\
79.1\end{array}$ & $\begin{array}{l}89.1 \\
73.7\end{array}$ \\
\hline
\end{tabular}

NF PWt. 1

$\begin{array}{lllllllllllllllll}-.3 & .4 & -.4 & .1 & .8 & 1.3 & .5 & .6 & .5 & .7 & .8 & 1.0 & 1.3 & .9 & .5 & .3 \\ & & .4 & .1 & .2 & -.2 & .1 & .4 & .0 & -1.3 & -2.6 & -1.3 & -3.1 & -2.7 & -3.2 & -3.5\end{array}$

$\begin{array}{rrrrrrrrrrrrrrrr}-2.3 & -1.3 & -2.5 & -3.4 & -.3 & 1.1 & .5 & -.4 & -.7 & -2.1 & -1.5 & 1.2 & -3.4 & -2.7 & -1.9 & -1.0 \\ & & -2.5 & -1.7 & -.1 & -2.3 & -1.8 & -1.3 & -2.4 & -4.2 & -4.4 & -3.1 & -5.2 & -4.2 & -4.8 & -5.0\end{array}$

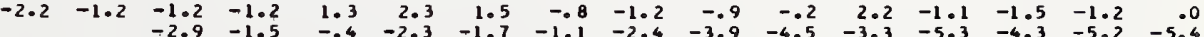

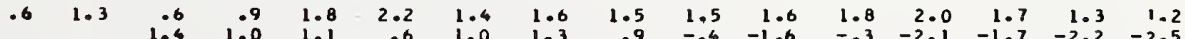

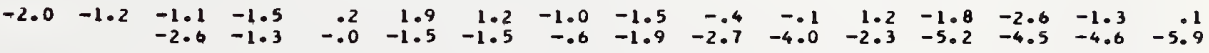

$\begin{array}{rrrrrrrrrrrrrrrr}-1.1 & -.0 & -.1 & .1 & 2.6 & 3.5 & 2.7 & .4 & -. .0 & .3 & .9 & 3.2 & .1 & -.4 & -.0 & 1.1 \\ & & -1.8 & -.4 & .7 & -1.3 & -.6 & .1 & -1.2 & -2.8 & -3.3 & -2.1 & -4.1 & -3.2 & -4.1 & -4.2\end{array}$

TABL E. OF OGTAVE Band POWER LEVELS

$\begin{array}{rrrrrrrrrrr}1 & C & 31.5 & 63 & 125 & 250 & 500 & 1 K & 2 K & 4 K & 8 K \\ 101.4 & 107.3 & 09.3 & 104.7 & 94.5 & 98.7 & 95.8 & 97.9 & 95.1 & 94.8 & 87.9 \\ 103.5 & 107.2 & .0 & 104.4 & 96.0 & 98.4 & 96.9 & 97.4 & 94.6 & 96.0 & 92.1 \\ 2.1 & -.1 & .0 & -. .6 & 1.5 & -. .3 & 1.1 & -.5 & -.5 & 1.2 & 4.2\end{array}$

FAR FIELO PWL

FAR FIELO METHODOLOGY PWL 


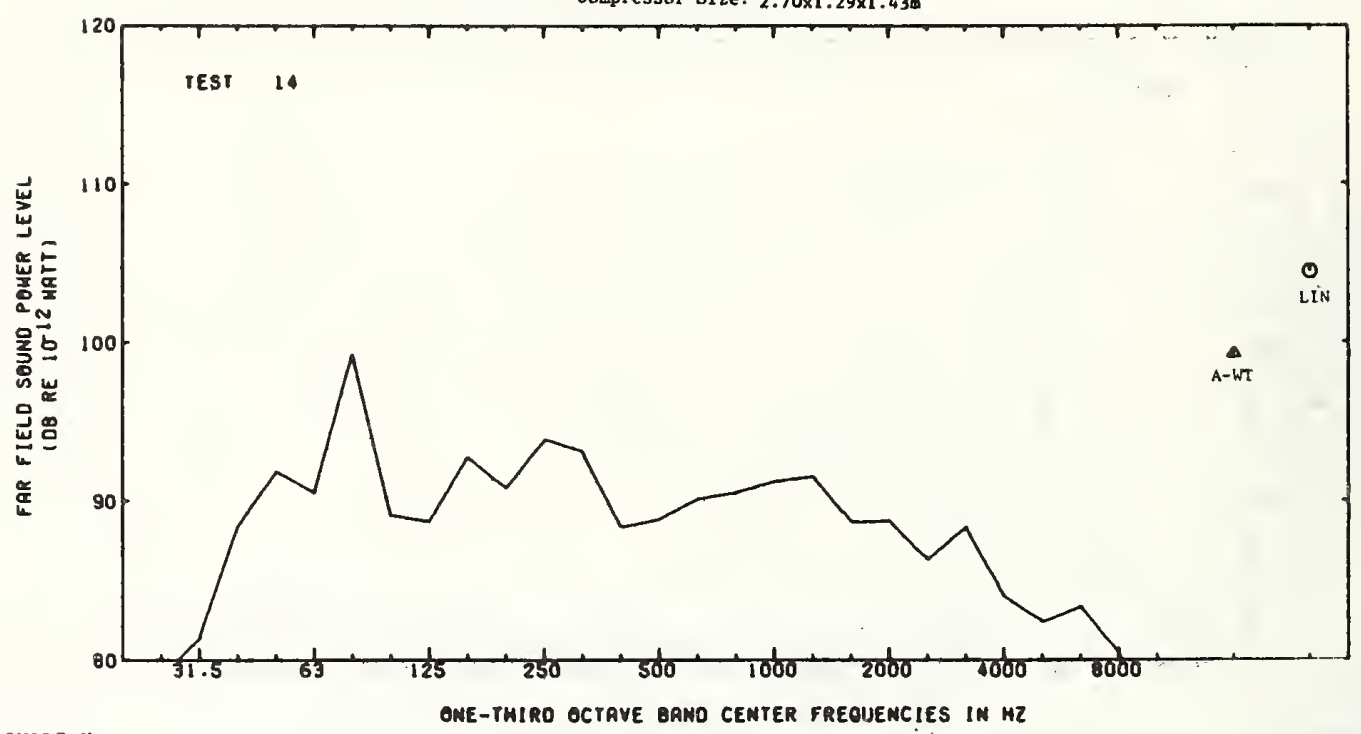

SIGNAL QUALITY $\begin{array}{lllllllllllllllllllllllllllll}\text { FAR FIELD } & \mathbf{4} & \mathbf{N} & \mathbf{N} & \mathbf{T} & \mathbf{T} & \mathbf{T} & \mathbf{N} & \mathbf{N} & \mathbf{T} & \mathbf{N} & \mathbf{T} & \mathbf{T} & \mathbf{N} & \mathbf{N} & \mathbf{N} & \mathbf{N} & \mathbf{F} & \mathbf{N} & \mathbf{F} & \mathbf{F} & \mathbf{F} & \mathbf{F} & \mathbf{F} & \mathbf{F} & \mathbf{F} & \mathbf{F} & \mathbf{F} \\ \text { NEAR FIELD } & \mathbf{F} & \mathbf{N} & \mathbf{N} & \mathbf{T} & \mathbf{T} & \mathbf{T} & \mathbf{N} & \mathbf{N} & \mathbf{T} & \mathbf{N} & \mathbf{T} & \mathbf{T} & \mathbf{N} & \mathbf{N} & \mathbf{N} & \mathbf{N} & \mathbf{N} & \mathbf{N} & \mathbf{N} & \mathbf{F} & \mathbf{F} & \mathbf{F} & \mathbf{F} & \mathbf{F} & \mathbf{F} & \mathbf{F} & \mathbf{F}\end{array}$ ITST NUMUER 14
COMPRE SSOR SURFACE ARFA=14.85 SO. METRES

CONFORMAL SURFACE AREA $(R=1 M)=42.63$ SO. METRES

WE IGHI ING-LOW FREOUENCY
HIGH FREOUENCY
FAR FIELO PWL
NF PWL 1
NF PWL 2
NF PWL 3
NF PWL 6
NF PWL 7
NF PWL 8

\begin{tabular}{|c|c|c|c|c|c|c|c|c|c|c|c|c|c|c|c|}
\hline $\begin{array}{c}4 \\
99.3\end{array}$ & $\begin{array}{c}\text { LIN } \\
104.5\end{array}$ & $\begin{array}{l}20 \\
500 \\
85.6 \\
88.9\end{array}$ & $\begin{array}{c}25 \\
630 \\
79.0 \\
90.2\end{array}$ & $\begin{array}{c}\text { EIGHT EO } \\
31.5 \\
800 \\
81.4 \\
90.6\end{array}$ & $\begin{array}{l}\text { SOUND } \\
40 \\
1 \mathrm{~K} \\
88.4 \\
91.3\end{array}$ & $\begin{array}{l}\text { POHER } \\
50 \\
1.2 K \\
91.9 \\
91.6\end{array}$ & $\begin{array}{l}\text { LEVE } \\
63 \\
1.6 K \\
90.5 \\
88.7\end{array}$ & $\begin{array}{r}E L 108 \\
80 \\
2 k \\
59.3 \\
88.8\end{array}$ & $\begin{array}{c}\text { RE } 1 \text { PI } \\
100 \\
2.5 K \\
89.1 \\
86.3\end{array}$ & $\begin{array}{l}\text { COHATT } \\
125 \\
3.1 \mathrm{~K} \\
88.7 \\
88.4\end{array}$ & $\begin{array}{l}160 \\
4 K \\
92.8 \\
84.0\end{array}$ & $\begin{array}{l}200 \\
5 K \\
90.8 \\
82.4\end{array}$ & $\begin{array}{c}250 \\
6.3 k \\
93.9 \\
83.4\end{array}$ & $\begin{array}{l}315 \\
8 K \\
93.1 \\
80.4\end{array}$ & $\begin{array}{l}400 \\
10 K \\
88.4 \\
75.1\end{array}$ \\
\hline 99.4 & 105.2 & $\begin{array}{l}89.3 \\
89.3\end{array}$ & $\begin{array}{l}85.6 \\
90.4\end{array}$ & $\begin{array}{l}85.4 \\
91.0\end{array}$ & $\begin{array}{l}89.8 \\
90.9\end{array}$ & $\begin{array}{l}93.8 \\
91.6\end{array}$ & $\begin{array}{l}91.5 \\
89.5\end{array}$ & $\begin{array}{r}100.1 \\
88.9\end{array}$ & $\begin{array}{l}90.0 \\
86.3\end{array}$ & $\begin{array}{l}80.1 \\
88.5\end{array}$ & $\begin{array}{l}94.7 \\
84.6\end{array}$ & $\begin{array}{l}91.9 \\
82.2\end{array}$ & $\begin{array}{l}94.3 \\
83.2\end{array}$ & $\begin{array}{l}93.5 \\
82.1\end{array}$ & $\begin{array}{l}91.2 \\
77.6\end{array}$ \\
\hline 98.7 & 104.2 & $\begin{array}{l}86.7 \\
90.0\end{array}$ & $\begin{array}{l}79.5 \\
90.1\end{array}$ & $\begin{array}{l}82.1 \\
90.7\end{array}$ & $\begin{array}{l}88.8 \\
90.3\end{array}$ & $\begin{array}{l}94.1 \\
91.3\end{array}$ & $\begin{array}{l}91.4 \\
89.0\end{array}$ & $\begin{array}{l}99.7 \\
87.8\end{array}$ & $\begin{array}{l}87.9 \\
85.3\end{array}$ & $\begin{array}{l}87.0 \\
87.7\end{array}$ & $\begin{array}{l}91.8 \\
83.2\end{array}$ & $\begin{array}{l}89.2 \\
79.7\end{array}$ & $\begin{array}{l}91.6 \\
81.3\end{array}$ & $\begin{array}{l}90.5 \\
76.8\end{array}$ & $\begin{array}{l}87.7 \\
69.9\end{array}$ \\
\hline 99.0 & 104.4 & $\begin{array}{l}86.9 \\
89.6\end{array}$ & $\begin{array}{l}80.2 \\
90.2\end{array}$ & $\begin{array}{l}83.1 \\
91.1\end{array}$ & $\begin{array}{l}89.7 \\
90.5\end{array}$ & $\begin{array}{l}94.0 \\
91.4\end{array}$ & $\begin{array}{l}91.3 \\
89.6\end{array}$ & $\begin{array}{r}100.0 \\
88.4\end{array}$ & $\begin{array}{l}88.7 \\
86.2\end{array}$ & $\begin{array}{l}87.1 \\
87.7\end{array}$ & $\begin{array}{l}90.6 \\
83.3\end{array}$ & $\begin{array}{l}90.3 \\
80.2\end{array}$ & $\begin{array}{l}92.1 \\
82.2\end{array}$ & $\begin{array}{l}91.0 \\
77.2\end{array}$ & $\begin{array}{l}88.3 \\
70.6\end{array}$ \\
\hline 00.4 & 106.2 & $\begin{array}{l}90.1 \\
90.4\end{array}$ & $\begin{array}{l}86.3 \\
91.4\end{array}$ & $\begin{array}{l}86.2 \\
92.0\end{array}$ & $\begin{array}{l}90.6 \\
92.0\end{array}$ & $\begin{array}{l}94.7 \\
92.6\end{array}$ & $\begin{array}{l}92.7 \\
90.5\end{array}$ & $\begin{array}{r}101.3 \\
89.8\end{array}$ & $\begin{array}{l}90.8 \\
87.2\end{array}$ & $\begin{array}{l}90.9 \\
89.4\end{array}$ & $\begin{array}{l}95.5 \\
85.5\end{array}$ & $\begin{array}{l}92.7 \\
83.2\end{array}$ & & & $\begin{array}{l}92.0 \\
78.4\end{array}$ \\
\hline 99.2 & 103.7 & $\begin{array}{l}87.1 \\
90.0\end{array}$ & $\begin{array}{l}80.2 \\
90.2\end{array}$ & $\begin{array}{l}82.3 \\
90.7\end{array}$ & $\begin{array}{l}89.5 \\
90.4\end{array}$ & $\begin{array}{l}94.9 \\
90.9\end{array}$ & $\begin{array}{l}91.2 \\
89.6\end{array}$ & $\begin{array}{l}99.6 \\
88.5\end{array}$ & $\begin{array}{l}89.0 \\
85.9\end{array}$ & $\begin{array}{l}88.1 \\
88.0\end{array}$ & $\begin{array}{l}90.4 \\
83.7\end{array}$ & $\begin{array}{l}90.0 \\
80.1\end{array}$ & & $\begin{array}{l}90.8 \\
76.7\end{array}$ & $\begin{array}{l}88.6 \\
70.4\end{array}$ \\
\hline 0.2 & 105.6 & $\begin{array}{l}88.1 \\
90.7\end{array}$ & $\begin{array}{l}81.4 \\
91.3\end{array}$ & $\begin{array}{l}84.2 \\
92.2\end{array}$ & $\begin{array}{l}90.8 \\
91.6\end{array}$ & $\begin{array}{l}95.0 \\
92.5\end{array}$ & $\begin{array}{l}92.5 \\
90.8\end{array}$ & $\begin{array}{r}101.3 \\
89.5\end{array}$ & $\begin{array}{l}89.8 \\
87.4\end{array}$ & $\begin{array}{l}88.3 \\
88.8\end{array}$ & $\begin{array}{l}91.8 \\
84.4\end{array}$ & $\begin{array}{l}91.5 \\
81.3\end{array}$ & $\begin{array}{l}93.2 \\
83.3\end{array}$ & $\begin{array}{l}92.1 \\
78.4\end{array}$ & $\begin{array}{l}89.4 \\
71.8\end{array}$ \\
\hline
\end{tabular}

NF PWL 1

NF PWL ?

NF P H 3

NF PWL 6

NF PML 7

NF PWL 8

FAR FIELO PML

FAR FIELD METHODOLOCY PHL

DEVIATIONS OF FF METH FROM FF

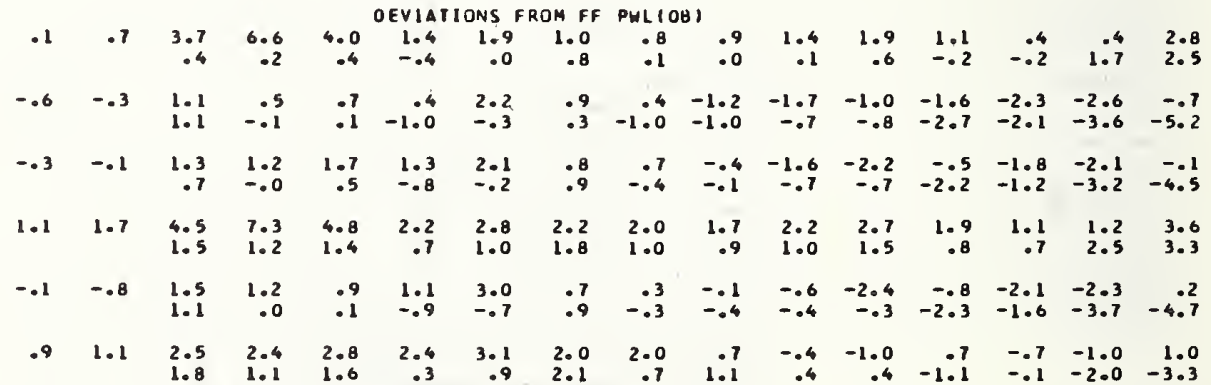

table of octave bano poner levels

$\begin{array}{rrrrrrrrrrr}1 & C & 31.5 & 63 & 125 & 250 & 500 & 1 K & 2 K & 4 K & 8 K \\ 99.3 & 104.7 & 69.6 & 100.5 & 95.4 & 97.6 & 94.0 & 96.0 & 92.8 & 90.5 & 85.6 \\ 100.9 & 105.1 & .0 & 101.0 & 91.3 & 96.8 & 96.1 & 94.7 & 93.3 & 92.2 & 92.3 \\ 1.6 & .4 & .0 & .5 & 1.9 & -.8 & 2.1 & -1.3 & .5 & 1.7 & 6.7\end{array}$




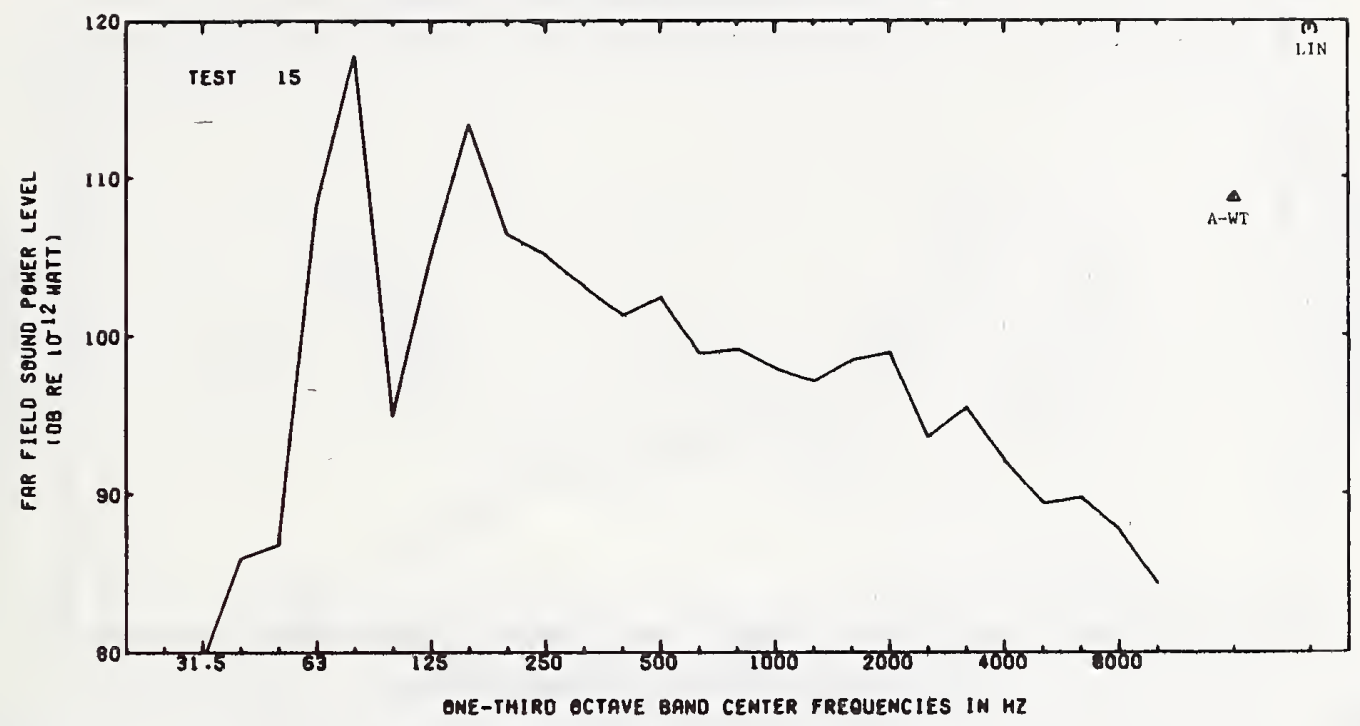

SIGNAL QUALITY

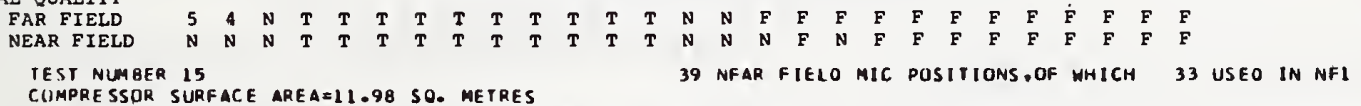

WE IGHTING-LOY FREQUENCY HIGH FREQUENCY

FAR FIELO PML

NF PWL I

NF PWL 2

NF PWL 3

NF PUL 6

NF PWL 7

NF P ill 6

NF PML 1

NF P WL 2

NF P W 3

NF PW 6

NF PWL 7

NF PUL 8

FAR FIELO PUL

FAR FIELD HE THODOLOCY PIL

DEVIATIONS OF FE NETH FRUM FF

\section{HEIGHTEO SOUNO PUMER LEVELIOB RE 1 PICOMATT:}

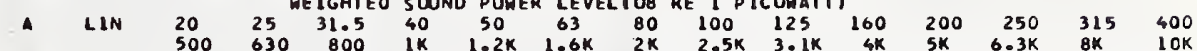

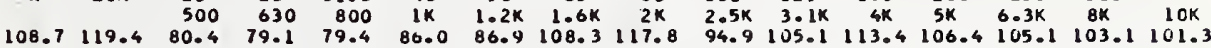

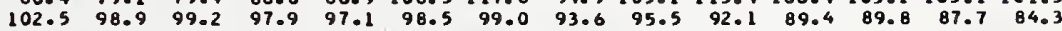

$109.8 \quad 120.0 \quad 78.9 \quad 79.0 \quad 82.0 \quad 88.4 \quad 87.5 \quad 107.1 \quad 118.7 \quad 95.4 \quad 104.3 \quad 113.9 \quad 106.7 \quad 106.4 \quad 103.6 \quad 102.9$ $\begin{array}{llllllllllllllll}103.9 & 100.4 & 100.6 & 100.2 & 98.1 & 99.4 & 99.3 & 95.1 & 97.5 & 93.7 & 91.2 & 90.9 & 88.2 & 84.0\end{array}$

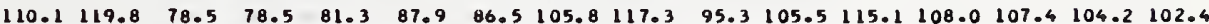
$105.1100 .299 .9 \quad 99.6 \quad 98.1 \quad 100.0100 .1 \quad 95.197 .293 .991 .391 .498 .9 \quad 85.7$

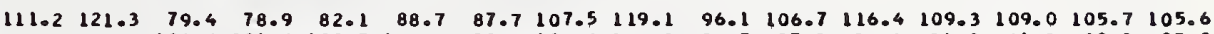
$\begin{array}{lllllllllllllll}106.6 & 101.6 & 100.5 & 100.4 & 98.6 & 100.3 & 100.2 & 95.7 & 97.9 & 94.3 & 91.3 & 91.9 & 89.0 & 85.2\end{array}$

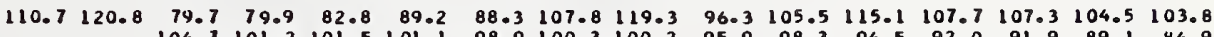
$\begin{array}{lllllllllllllll}104.7 & 101.2 & 101.5 & 101.1 & 98.9 & 100.3 & 100.2 & 95.9 & 98.3 & 94.5 & 92.0 & 91.9 & 89.1 & 44.9\end{array}$

$\begin{array}{lllllllllllllllll}110.7 & 120.6 & 79.3 & 79.0 & 81.4 & 88.7 & 88.0 & 107.1 & 118.7 & 94.7 & 106.0 & 115.7 & 108.5 & 108.6 & 104.5 & 104.2\end{array}$ $105.8101 .1 \quad 100.2 \quad 100.2 \quad 98.9100 .1100 .0 \quad 95.098 .199 .791 .191 .6 \quad 89.3 \quad 64.6$

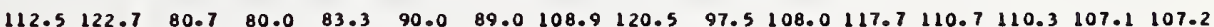

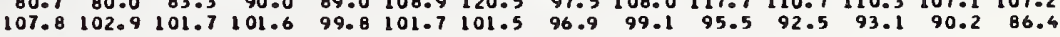

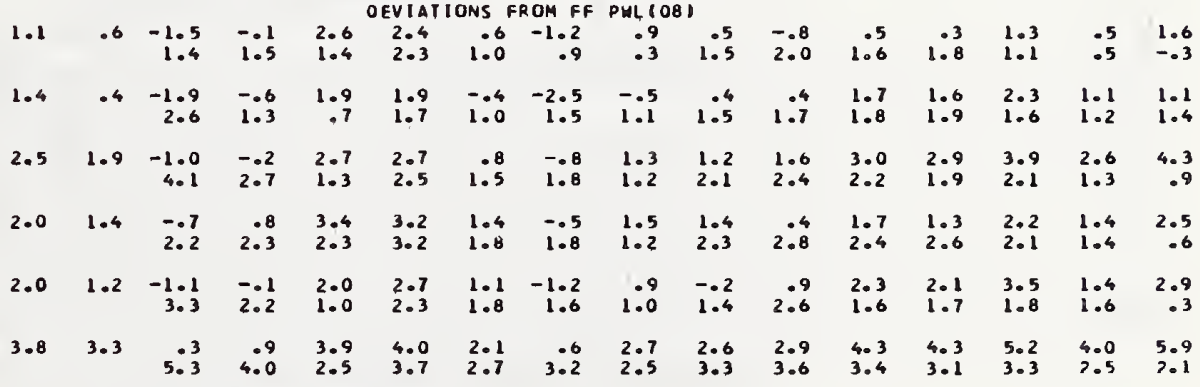

IABLE OF OCTAVE BANO POHER LEVELS

$\begin{array}{lllllllllll}A & C & 31.5 & 63 & 125 & 250 & 500 & 1 K & 2 k & 4 k & 8 k\end{array}$

$\begin{array}{lllllllllll}108.7 & 120.0 & 87.5 & 118.3 & 114.1 & 109.8 & 105.9 & 102.9 & 102.4 & 97.8 & 92.6\end{array}$

$\begin{array}{lllllllllll}110.9 & 120.5 & .0 & 119.0 & 113.4 & 109.1 & 108.2 & 104.8 & 102.6 & 100.6 & 98.1\end{array}$

$\begin{array}{lllllllllll}2.2 & .5 & .0 & .7 & -.7 & -.7 & 2.3 & 1.9 & .2 & 2.8 & 5.5\end{array}$ 


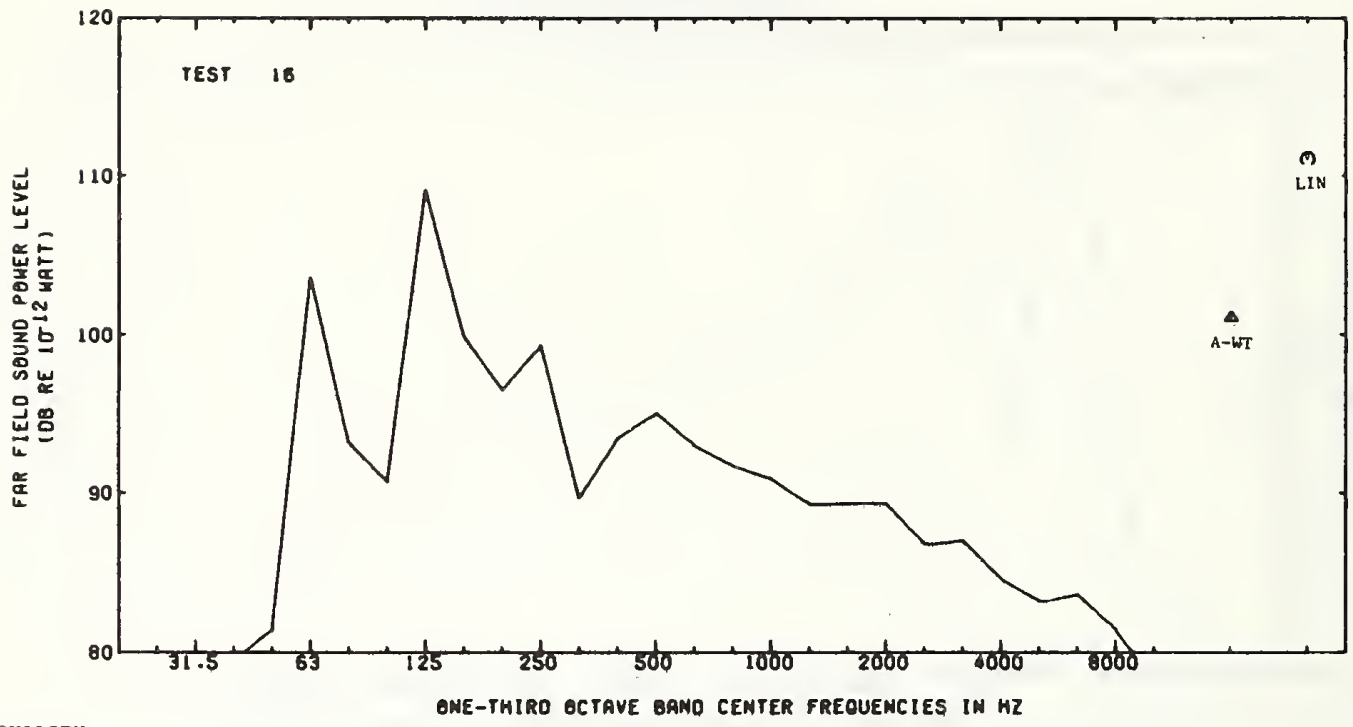

SIGNAL QUALITY

$\begin{array}{lllllllllllllllllllllllllllll}\text { FAR FIELD } & \mathbf{T} & \mathbf{N} & \mathbf{F} & \mathbf{N} & \mathbf{T} & \mathbf{T} & \mathbf{T} & \mathbf{T} & \mathbf{T} & \mathbf{N} & \mathbf{N} & \mathbf{N} & \mathbf{N} & \mathbf{F} & \mathbf{N} & \mathbf{F} & \mathbf{F} & \mathbf{F} & \mathbf{F} & \mathbf{F} & \mathbf{F} & \mathbf{F} & \mathbf{F} & \mathbf{F} & \mathbf{F} & \mathbf{F} & \mathbf{F} \\ \text { NEAR FIELD } & \mathbf{N} & \mathbf{N} & \mathbf{F} & \mathbf{N} & \mathbf{T} & \mathbf{T} & \mathbf{T} & \mathbf{T} & \mathbf{T} & \mathbf{N} & \mathbf{N} & \mathbf{N} & \mathbf{N} & \mathbf{F} & \mathbf{N} & \mathbf{F} & \mathbf{F} & \mathbf{F} & \mathbf{F} & \mathbf{F} & \mathbf{F} & \mathbf{F} & \mathbf{F} & \mathbf{F} & \mathbf{F} & \mathbf{F} & \mathbf{F}\end{array}$

TEST NUMBER 16

COMPRESSOR SURFACE AREA $=10.36$ SO. HETRES

39 NEAR FIELO MIC POSITIONS, OF HMICH 33 USEO IN NFI

CONFORMAL SURFACE AREA $(R=1 M)=34.67$ SO. METRES

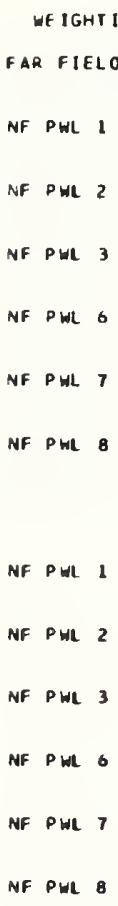

FAR FIELO PHL

FAR FLELO METHOOOL OGY PHL

OEVIATIONS OF FF METH FROM FF

\begin{tabular}{|c|c|c|c|c|c|c|c|c|c|c|c|c|c|c|c|}
\hline $\begin{array}{c}A \\
101.1\end{array}$ & 111.1 & $\begin{array}{l}20 \\
500 \\
78.7 \\
95.1\end{array}$ & $\begin{array}{c}25 \\
630 \\
77.4 \\
93.0\end{array}$ & $\begin{array}{l}\text { E LHTEO } \\
31.5 \\
800 \\
79.0 \\
91.7\end{array}$ & $\begin{array}{l}\text { SOUNO } \\
40 \\
1 K \\
79.5 \\
90.9\end{array}$ & $\begin{array}{l}\text { POWE } \\
50 \\
1.2 K \\
81.5 \\
89.3\end{array}$ & $\begin{array}{c}\text { ER LEVE } \\
63 \\
1.6 K \\
103.7 \\
89.4\end{array}$ & $\begin{array}{c}80 \\
2 k \\
93.2 \\
89.4\end{array}$ & $\begin{array}{l}\mid E{ }_{10}^{1} p \\
100 \\
2.5 k \\
90.7 \\
86.8\end{array}$ & $\begin{array}{c}\text { ICOHAT } \\
125 \\
3.1 K \\
109.2 \\
87.1\end{array}$ & $\begin{array}{c}160 \\
4 k \\
99.9 \\
84.6\end{array}$ & $\begin{array}{l}200 \\
5 K \\
96.5 \\
83.2\end{array}$ & $\begin{array}{r}250 \\
6.31 \\
99.4 \\
83.7\end{array}$ & $\begin{array}{l}315 \\
8 K \\
89.7 \\
81.4\end{array}$ & $\begin{array}{l}400 \\
10 K \\
93.5 \\
78.3\end{array}$ \\
\hline 01.6 & 111.9 & $\begin{array}{l}74 . \\
96 .\end{array}$ & $\begin{array}{l}75.2 \\
93.8\end{array}$ & $\begin{array}{l}80.3 \\
92.4\end{array}$ & $\begin{array}{l}79 . \\
91 .\end{array}$ & $\begin{array}{l}82.5 \\
90.2\end{array}$ & $\begin{array}{r}104.5 \\
90.3\end{array}$ & & $\begin{array}{l}92.0 \\
87.4\end{array}$ & $\begin{array}{r}110.0 \\
87.5\end{array}$ & $\begin{array}{r}100.9 \\
85.2\end{array}$ & $\begin{array}{l}97.9 \\
83.1\end{array}$ & $\begin{array}{r}100.3 \\
83.9\end{array}$ & $\begin{array}{l}89.5 \\
81.1\end{array}$ & $\begin{array}{l}93.5 \\
77.4\end{array}$ \\
\hline 101.8 & 112.9 & $\begin{array}{l}68.7 \\
96.2\end{array}$ & $\begin{array}{l}72.6 \\
92.9\end{array}$ & $\begin{array}{l}76.0 \\
91.0\end{array}$ & $\begin{array}{l}78.0 \\
90.7\end{array}$ & $\begin{array}{l}80.8 \\
88.8\end{array}$ & $\begin{array}{r}103.9 \\
89.9\end{array}$ & & $\begin{array}{l}92.1 \\
87.2\end{array}$ & $\begin{array}{r}111.8 \\
86.9\end{array}$ & $\begin{array}{r}102.4 \\
85.0\end{array}$ & & & & $\begin{array}{l}92.9 \\
78.3\end{array}$ \\
\hline 102.4 & 113.7 & $\begin{array}{l}75.4 \\
97.0\end{array}$ & $\begin{array}{l}75.6 \\
93.3\end{array}$ & $\begin{array}{l}78.2 \\
91.6\end{array}$ & $\begin{array}{l}79.1 \\
91.3\end{array}$ & $\begin{array}{l}82.5 \\
89.5\end{array}$ & $\begin{array}{r}105.7 \\
90.6\end{array}$ & $\begin{array}{l}95.1 \\
89.9\end{array}$ & $\begin{array}{l}93.6 \\
87.6\end{array}$ & $\begin{array}{r}112.6 \\
87.7\end{array}$ & $\begin{array}{r}103.2 \\
85.8\end{array}$ & & & & $\begin{array}{l}93.8 \\
78.6\end{array}$ \\
\hline 102.8 & 113.0 & $\begin{array}{l}75.4 \\
97.2\end{array}$ & $\begin{array}{l}75.8 \\
94.7\end{array}$ & $\begin{array}{l}81.0 \\
93.3\end{array}$ & $\begin{array}{l}80.6 \\
92.2\end{array}$ & $\begin{array}{l}83.3 \\
91.1\end{array}$ & $\begin{array}{r}105.3 \\
91.2\end{array}$ & $\begin{array}{l}95.1 \\
90.6\end{array}$ & $\begin{array}{l}92.9 \\
88.3\end{array}$ & $\begin{array}{r}111.2 \\
88.5\end{array}$ & $\begin{array}{r}102.0 \\
86.1\end{array}$ & & $\begin{array}{r}101.3 \\
84.8\end{array}$ & & $\begin{array}{l}94.5 \\
78.3\end{array}$ \\
\hline 101. & 112. & $\begin{array}{l}73.8 \\
96.2\end{array}$ & $\begin{array}{l}74.6 \\
92.8\end{array}$ & $\begin{array}{l}78.4 \\
91.4\end{array}$ & $\begin{array}{l}79.1 \\
91.2\end{array}$ & $\begin{array}{l}81.6 \\
89.8\end{array}$ & $\begin{array}{r}104.2 \\
90.6\end{array}$ & $\begin{array}{l}93.8 \\
89.9\end{array}$ & $\begin{array}{l}92.3 \\
87.6\end{array}$ & $\begin{array}{r}111.6 \\
88.2\end{array}$ & $\begin{array}{r}102.2 \\
84.9\end{array}$ & & & & $\begin{array}{l}93.3 \\
78.6\end{array}$ \\
\hline 103 & 113.0 & $\begin{array}{l}77.1 \\
98.3\end{array}$ & $\begin{array}{l}77.0 \\
94.6\end{array}$ & $\begin{array}{l}79.6 \\
92.8\end{array}$ & $\begin{array}{l}80.4 \\
92.5\end{array}$ & $\begin{array}{l}83.9 \\
90.7\end{array}$ & $\begin{array}{r}107.2 \\
91.9\end{array}$ & $\begin{array}{l}96.6 \\
91.1\end{array}$ & $\begin{array}{l}95.1 \\
88.9\end{array}$ & $\begin{array}{r}113.9 \\
89.0\end{array}$ & $\begin{array}{r}104.5 \\
87.1\end{array}$ & $\begin{array}{r}100.8 \\
85.2\end{array}$ & $\begin{array}{r}101.3 \\
85.1\end{array}$ & $\begin{array}{l}91.5 \\
82.8\end{array}$ & 80.0 \\
\hline
\end{tabular}

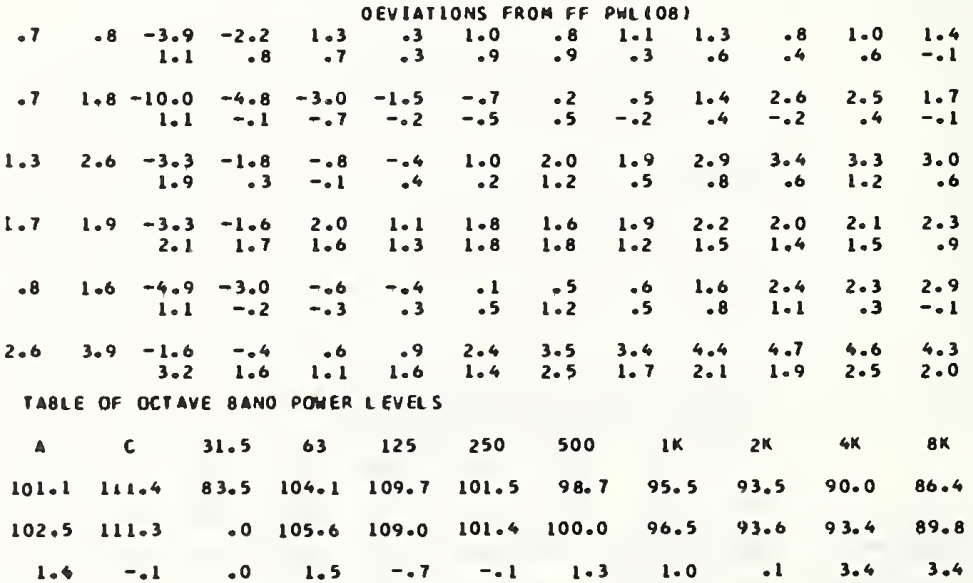




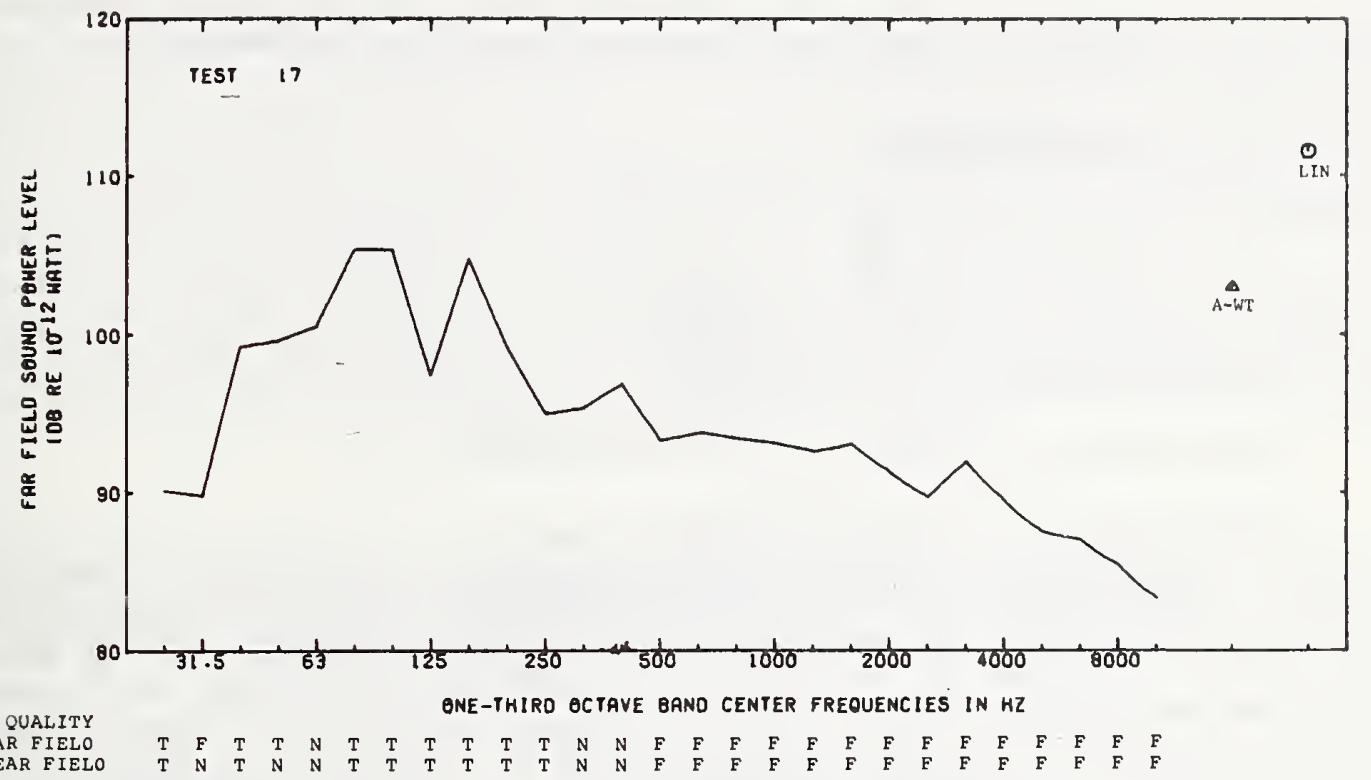

IEST NUMBER 17

CIIMPRESSUR SURFACE AREA $=11.02$ SO. METRES

47 NEAR FIELO MIC PUSITIUNS, OF HHICH 33 USEO IN NFI CONFORMAL, SURFACE AREA $(R=1 M)=35.40$ SO. METRES

WE IGHTING LOW FREQUENCY
FAR FIELO PHL FREOUENCY
NF PWL 1
NF PWL 2
NF PWL 3
NF PWL 6
NF PWL 7
NF PHL 6

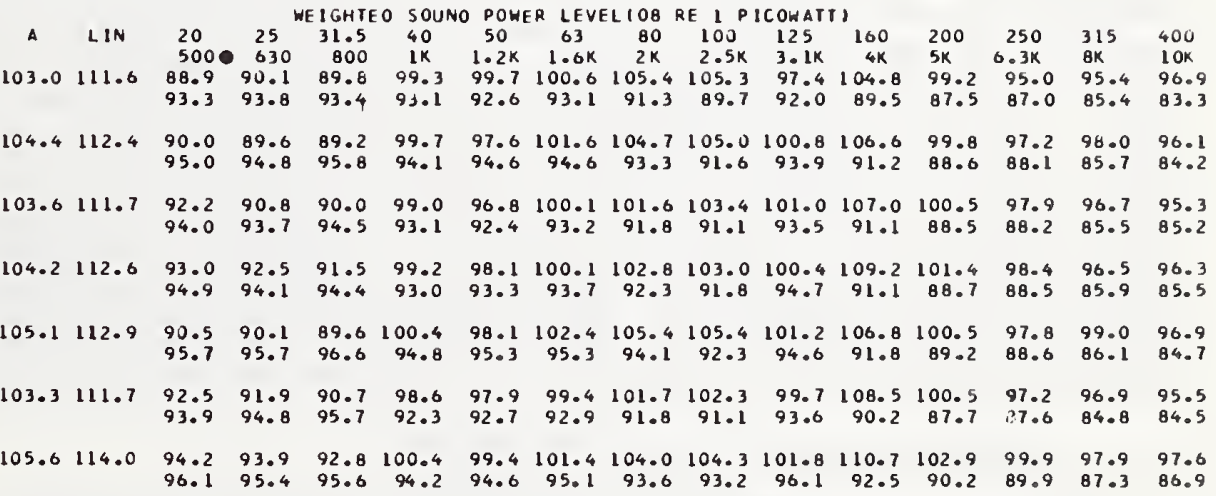

NF PHL 1

NF PWL 2

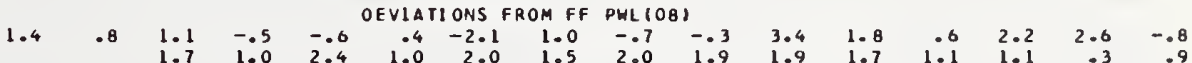
$\begin{array}{llllllllllllllllll}.6 & .1 & 3.3 & .7 & .2 & -.3 & -2.9 & -.5 & -3.8 & -1.9 & 3.6 & 2.2 & 1.3 & 2.9 & 1.3 & -1.6\end{array}$ NF PHL 3

NF PWL 6

NF PWL 7

NF PHL 8

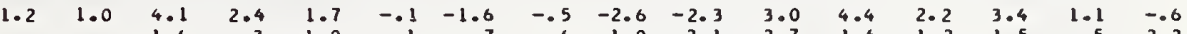

$\begin{array}{llllllllllllllllll}2.1 & 1.3 & 1.6 & -.0 & -.2 & 1.1 & -1.6 & 1.8 & -.0 & .1 & 3.8 & 2.0 & 1.3 & 2.8 & 3.6 & -.0\end{array}$

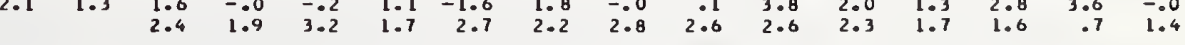

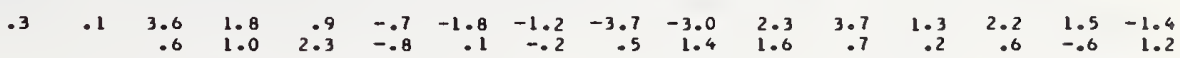

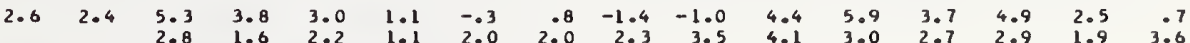
taBle of octaVe bano POHER LEVELS

FAR FIELO PWL

FAR FIELO METHOOOLOGY PHL OEVIATIONS OF FF METH FROM FF

$\begin{array}{rrrrrrrrrrr}4 & C & 31.5 & 63 & 125 & 250 & 500 & 1 K & 2 K & 4 K & 8 K \\ 103.0 & 111.9 & 100.2 & 107.4 & 108.4 & 101.7 & 99.7 & 97.8 & 96.4 & 94.8 & 90.3 \\ 104.2 & 112.2 & 99.0 & 107.6 & 109.3 & 100.6 & 100.9 & 97.1 & 95.4 & 96.6 & 93.9 \\ 1.2 & .3 & -1.2 & .2 & .9 & -1.1 & 1.2 & -.7 & -1.0 & 1.8 & 3.6\end{array}$




\section{DISCUGSION OF EXPERTMENTAL RESULTS}

4.1. Sound Pressure Level Data

There are two aspects of the sound pressure level data which are worthy of note with respect to compressor noise measurement -- a) directivity of compressor noise, and b) systematic variation of noise with elevation above the reflecting plane.

\subsubsection{Directivity of compressor noise}

A directivity index for a source may be defined as [8]

$$
D I(\theta)=I_{P}(\theta)-\bar{I}_{P}
$$

where $D I(\theta)$ is the directivity index at the angle $\theta$

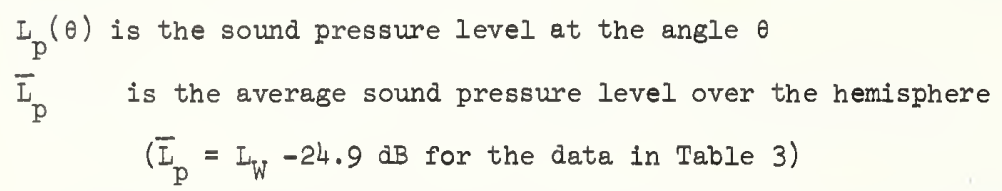

The range of maximum values of directivity index for A-weighted sound level for all tests was 1.8 $d B$ to $7.6 \mathrm{~dB}$. As might be expected, the larger values of directivity index were those associated with the larger machines. For comparison, the maximum directivity index of A-weighted level for the reference source was $1.5 \mathrm{~dB}$ while that for the tone source ranged from 6.9 to $11.1 \mathrm{~dB}$. The average value for the maximum directivity index of A- weighted level was 3.4 dB. indicating that compressors as a group are not substantially directive sources. Furthermore, because of the relatively small directivity of compressors in comparison with tonal sources, we are led to suspect that the A-weighted level of compressor noise is not strongly dominated by tones. As a result, it is expected that the average of a small number of measurements is likely to give reasonably good estimates of the true average level over the measurement surface. For 12 of the 17 samples the side exhibiting maximum noise level in near-field data was in the same direction as the direction of maximum level in the far-field. For two compressors, the direction of the far field maximum was within $+45^{\circ}$ of the direction of the side with maximum level. For three of the compressors - those with low directivity index -- the maxima of sound pressure level in the near and far field data occurred on aifferent sides. This may be reasonably attributed to second order effects due to the size of the different sides as well as the typical interference patterns in the near-field for these sources. In all three cases, the maximum directivity index occurs close to the ground in the region of the first interference maximum, so that small elevation positioning errors may contribute to a false identification of the side with the maximum noise. Furthermore, for compressors, the near-field maximum typicaliy occurs on a short side and the increased area associated with a long side may in fact lead to larger intensities in the far-field for the side with the highest value of average sound level times area. It is for these reasons that directivity patterns are only defined in the far field of the source where the directivity pattern is independent of measurement radius [8].

\section{1 .2 Systematic variation of noise with position}

It should be noted that both the far-field and near-field sound pressure level data exhibit stronger variation with height above the reflecting plane than with change of lateral position on the measurement surface. The effect is larger in the far-field data than in the near-field data. This is a direct consequence of two facts: a) interference patterns are a result of the radiation from two or more coherent sources spaced some distance apart and b) the principal coherent sources are any position on the compressor and its mirror image on the other side of the reflecting plane. This may be predicted if one assumes that all the principal radiators of sound are small and not strongly correlated to all other radiators.

This factor is important for a measurement methodology since it implies that averaging in the vertical direction in the far-field will be much more important than averaging in the horizontal direction, and also more important in the far field than in the near-field.

\subsection{Sound Power Level Data.}

Several noteworthy conclusions can be drawn from the sound power level data [Tables 5.1-5.17]. The first is that for each individual test, for the frequency range above which the signal quality indicates no background noise problems, to an upper frequency limit of about $2.0 \mathrm{kHz}$, the deviations of the near field calculations are predominantly positive and small. One means for summarizing this result is shown in Table 6 where the averages of these deviations for 17 compressors are shown. (Note: points corresponding to signal quality greater than 3 are excluded from the average). Also shown are 
the computed standard deviations of these deviations. The average deviation may be interpreted as an estimate of the average bias of the measurement methodology, while the standard deviation can be interpreted as a measure of the precision of the methodology. These results will be more fully discussed in the next section.

Second, the signal quality code, which classifies the variance of the signal as tone-like, noise-like or fluctuating, generally agrees with an estimate of the presence of a tone from qualitative examination of the spectrum (i.e., peaks in the spectrum correspond to T's in the signal. quality) except at frequencies above about $500 \mathrm{~Hz}$. Also the classification of variance in the near-field data typically indicates the same or lower variance than in the far-field data. These observations are interpreted as indicating that the propagation medium is not uniform during the period of observation at a single position ( 30 seconds) causing the interference pattern and thus the variance of the signal to change significantly during the observation period. The cause of uniformly high variances at high frequencies is probably due to the fact that tones, when they are present, are not steady, but rather, shift due to changes in source rotating speed. This change in frequency leads to significant changes in the interference pattern at high frequencies, which can produce large fluctuations in mean square pressure at the observation point. Alternately, unsteady propagation conditions over a region of a few wavelengths in dimension, caused by changing thermal and temperature conditions or changes in their gradients, can lead to the same effect.

Table 6.

Average deviation and standard deviation of average deviation of near fleld sound power level from far fleld sound power level for seventeen portable alr compressors. Six nearfleld calculation procedures (see text section 3.2 ) are shown plus almilar statistica for far field methodology.

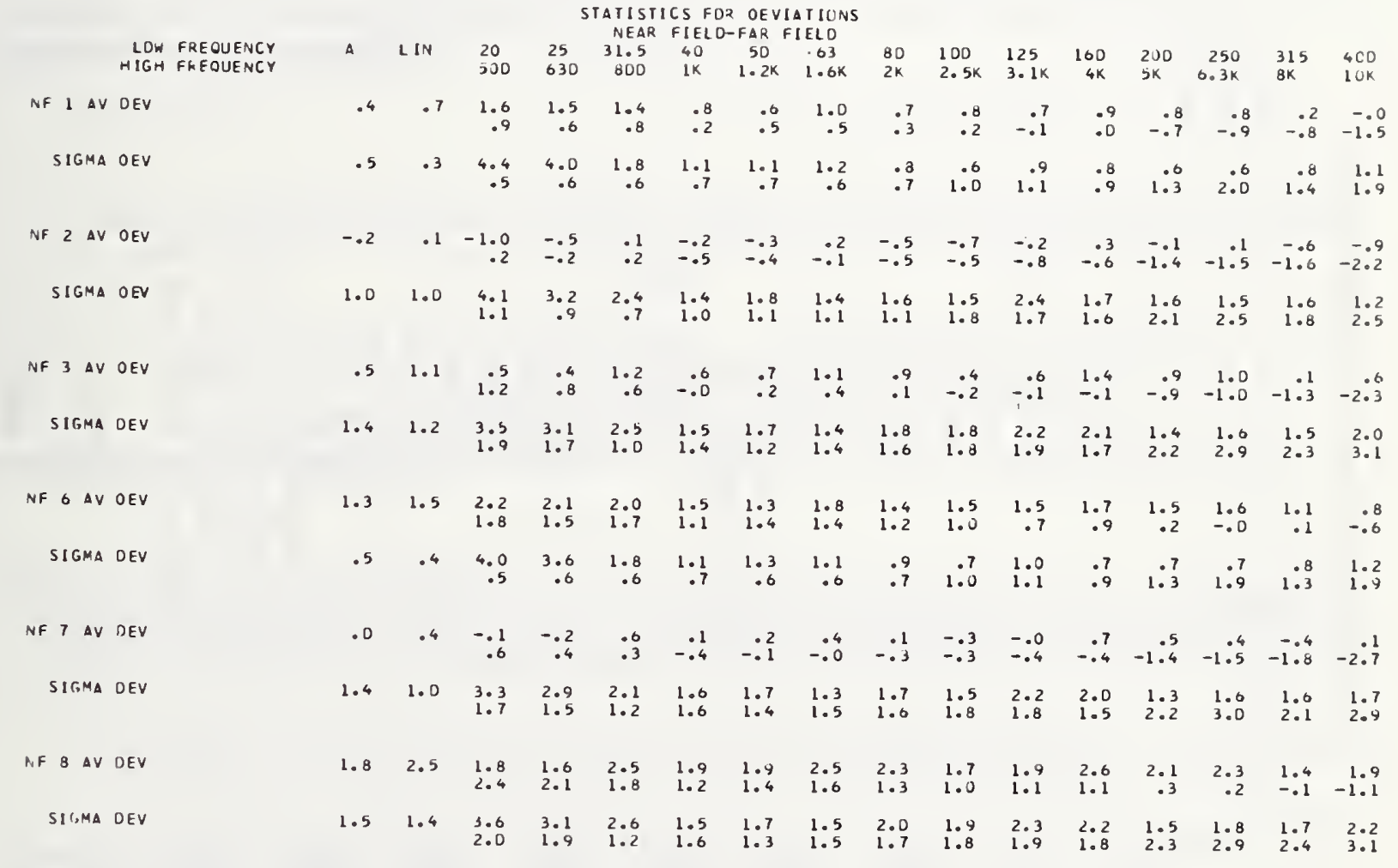

STATISTICS FOR DEVIATIUNS

FAR FIELD METHODOLDGY FAR FIELD

$\begin{array}{lcccccccccc}\Delta & C & 31.5 & 63 & 125 & 250 & 500 & 1 K & 2 K & 4 K & 8 K \\ 1.5 & .7 & -.3 & 1.2 & .9 & -.2 & 1.3 & -.3 & -.3 & 2.0 & 3.3 \\ .9 & 1.0 & .5 & 1.1 & 1.5 & 1.0 & .8 & 1.1 & 1.2 & 1.5 & 2.7\end{array}$




\section{ANATYSIS OF MEASUREMENT ERROR}

\subsection{Introduction}

10110w:

The errors associted with a measurement procedure may be broken into three major components as

1. Error associated with the test methodology;

2. Error associated with the measurement instrumentation;

3. Instrument operator error.

In this section we will discuss estimates of error for various sound power test methodologies on the basis of this experiment and attempt to put some bounds on the first two components of error, within the field test environment. We anticipate that the field test environment will include a test site and instrumentation meeting minimum requirements of the proposed ISO Draft engineering standard[2]. This implies a flat, hard-surfaced test site, and a commercially available, portable, precision sound level meter, operated under the supervision of a trained test engineer.

\subsection{Error in Sound Power Measurement Methodologies}

The deviation of sound power level estimates using near-field pressure levels from estimates using far-field pressure levels as presented in Table 6 and plotted in Figure 9 are surprisingly small. Further, they are consistent in suggesting that the estimate of sound power from near-field pressure measurements is higher than that of estimates from far field measurements, within the limits of sampling error. This consistent behavior of the data leads us to question whether or not an underlying physical principle in fact forces this behavior. A cursory review of the literature $[3,9,10]$ indicates that the topic of analytically relating near-field pressure to sound power has

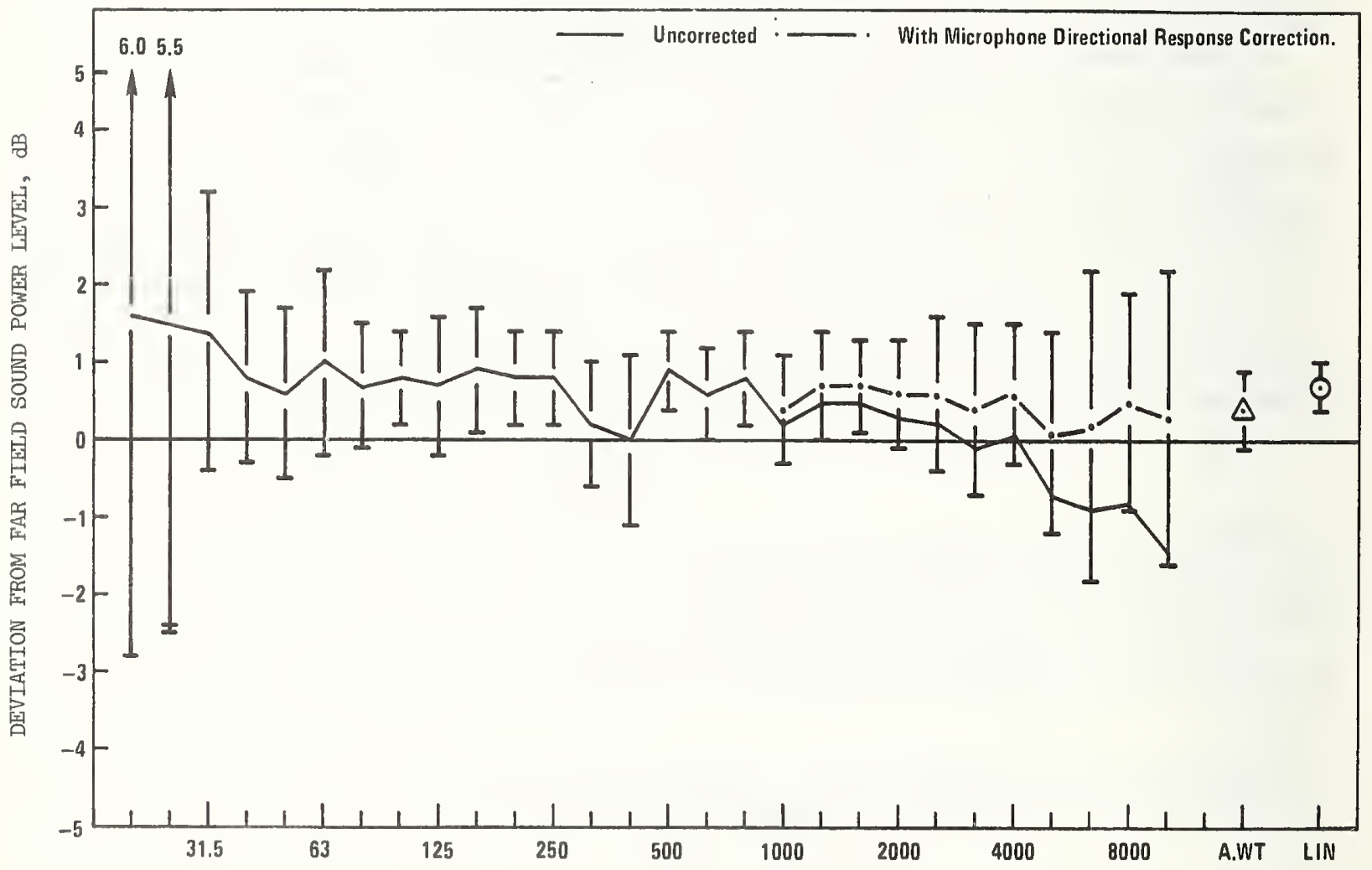

ONE-THIRD OCTAVE CENTER FREQUENCY, Hz.

Figure 9 Plot of average deviation of near field from far field sound power level 
received little or no attention. While a detailed analytic description is beyond the scope of this work, we are compelled, based on the strength of the data, to advance some hypotheses in this area both to aid in the interpretation of these data, and to suggest directions for further research.

We begin by introducing the true sound power, defined as [4]

$$
W=\int_{S_{0}} I(\underline{r}) \text { uŕn }(\underline{r}) d S
$$

where $W$ is the acoustic power radiated by all sources within $S_{0}$.

$\underline{I}(\underline{r})$ is the time average intensity vector at a position $\underline{r}$.

$\hat{n}(\underline{r})$ is the unit vector normal to the surface $S_{0}$ at the point $\underline{r}$.

An idealization of our measurement is given by

$$
W^{\prime \prime}=\int_{S} \frac{p^{2}(r)}{\rho c} d S
$$

where $p^{2}$ is the time-average squared pressure at the position $\underline{r}$.

Since the squared pressure is a scalar field, we introduce a third estimate of power for comparison, i.e.

$$
W^{\prime}=\int_{S}|\underline{I}(\underline{r})| d S
$$

where $|I|$ is the scalar magnitude of intensity.

Since $\quad|\underline{I}(\underline{r})| \geq \underline{I}(\underline{r}) \cdot \underline{n}(\underline{r})$,

It is clear that

$$
W^{\prime} \geq W
$$

Now, by analogy with the case of geometrical optics, we have in the geometric acoustic limit (i.e., vanishingly short wavelengths for incoherent noise sources ) we have that [11]

$$
|\underline{I}(\underline{r})| \underset{k r \rightarrow \infty}{\longrightarrow} \frac{p^{2}(\underline{r})}{\rho c}
$$

where $\quad k=\frac{2 \pi}{\lambda}$ is the acoustic wave number ( $\lambda$ is the wavelength of sound)

$r$ is the distance to the nearest source

Thus we may write

$$
W^{\prime \prime} \simeq W^{\prime} \geq W
$$

Where $\simeq$ indicates asymtotically equal to. Thus at least In the geometric acoustic (high frequency) limit, the near-field and far-field estimates of power for incoherent noise sources are asymtotic to an upper bound estimate of the true power. The degree of over-estimation is determined by the degree to which the shape of the measurement surface $S_{0}$ conforms to the surface of wave fronts from the source.

Our concern of course is how this description holds as wavelength increases. A cursory review of the data suggests the hypothesis for free field over reflecting plane determinations that

$$
W^{\prime \prime} \geq W
$$

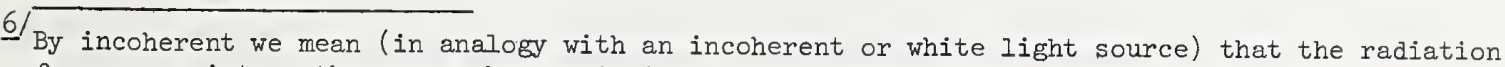
from any point on the source has vanishingly small temporal correlation with that from any other source point, and its temporal auto correlation approaches a delta function in time, such limiting processes being taken in a manner which yields a finite power output. 


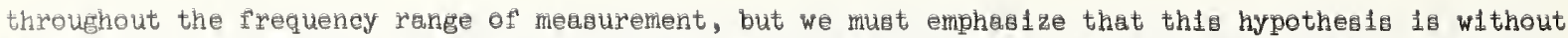

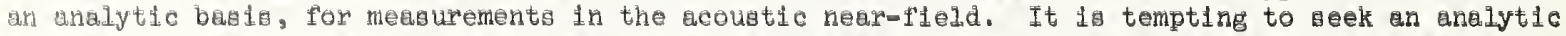
Justiflcation for this hypothesis based on a comparison of mean gquarea pressure with the acalar megnitude of intens1ty, but we must edd that auch attempts are met with considerable analytic difficulty for all but the simplest sources. For exemple, consiaeration of the simple monopole and dipole sources in free space yiela

$$
\frac{p^{2}(\underline{r})}{\rho c} \geq|\underline{I}(\underline{r})|
$$

for all frequencles, but a similar relationship for more complex sources has not been 1 dentified. Thus, though our data are very encouraging, we must omphasize at this point that there is no firm analytic basis for near-field sound power determinations.

With thid preamble, let us now turn to the question of differences between an estimate of power and true power. Equations 5.4 through 5.6 suggest the following partitioning of the error (as distinct from that proposed by Hubner [3])

$$
\begin{array}{ll} 
& \frac{W^{\prime \prime}}{W}=\delta_{s} \cdot \delta_{n}(\mathrm{kr}) \\
\text { Where } & \delta_{\mathrm{s}} \equiv \frac{\mathrm{W}^{\prime}}{\mathrm{W}} \\
& \delta_{\mathrm{n}}(\mathrm{kr}) \equiv \frac{\mathrm{W}^{\prime \prime}}{\mathrm{W}^{\prime}}
\end{array}
$$

The term $\delta$ is associated princlpally with the shape of the measurement surface relative to the time-average wavefront shape. Based on Huygens princlple, we expect this term to be nominally independent of the "radius" of a measurement surface, at least for nearly conformal measurement surfaces. The term $\delta_{n}(\mathrm{kr})$ is a frequency dependent term which is a measure of how the change in mean square pressure due to spreading differs from inverse square law. Thus, if the source does produce an acoustic near field, its contributions will occur principally in this term.

From equation 5.4, the term $\delta$ is seen to be always greater than or equal to unity. An estimate of the magnitude of the term $\delta_{n}(\mathrm{kr})$ is unavallable at this time except at high frequencies where eq. 5.5 indicates that it assymtotically approaches unity.

Unt1l this point, this discussion has lgnored the effect of spatial sampling on the measurement result. To consider 1ts effect we intrgduce a third estimate of sound power defined as

$$
W^{\prime \prime}=\sum_{1=1}^{N} s_{1} \frac{p^{2}(1)}{\rho c}
$$

where $S_{i}$ is the element of measurement surface area associated with the ith measurement of mean square pressure. If the measurement is designed so that the areas $\mathrm{S}_{i}$ are equal, then

$$
\mathrm{S}_{0}=\mathrm{N} \mathrm{S}_{\mathrm{i}}
$$

and

$$
W^{\prime} \cdots=\frac{S_{0}}{\rho c} \frac{1}{N} \sum_{i=1}^{N} p^{2}(i)
$$

In this form it is clear that the intent of the spatial sampling should be to produce an unbiased estimate of the average mean squared pressure over the measurement surface. Thus the expected value of W'" which results from an unbiased sampling plan will equal W". Thus we are led to expect that the effect of point sampling will be to introduce an imprecision in the measurement rather than a true bias. Further, the magnitude of this imprecision should be directly proportional to a function of the variance over the measurement surface and inversly proportional to a function of the number of uncorrelated measurement points used.

Returning to the data in Table 6, we note that all of the above comments are supported in detail. First consider the comparison between NF 1 compared with NF 6 which represents the most sensitive test of the difference between a conformal versus a rectangular surface. Above $250 \mathrm{~Hz}$, NF6 is a very consistent $0.9 \mathrm{~dB}$ high with a standard deviation of $0.8 \mathrm{~dB}$ or less through most of this range. $\mathrm{A}$ t-test indicates that this difference is significant at or above the $99 \%$ level. Thus we conclude that the difference between the procedures is statistically significant with the non-conformal surface producing the higher estimate, and thus that the term $\delta$ is greater than unity as expected. In Figure 10, the data for NFI is plotted versus frequency including the microphone directional response correction suggested in Section 2.3. Here we see a trend for the near field sound power estimate on the conformal surface to asymtotically approach the far field power with Increasing frequency as suggested by eq. 5.6, but further, moving toward lower frequencles, the upward blas becomes statistically significant. 
Thus we are led to a corollary of the hypothesis of eq. 5.7 , that

$$
E\left(W^{\prime \prime \prime}\right) \geq W
$$

i.e., the expected value of a sound power estimate based on sound pressure measurements is greater than or equal to the true sound power within the limits of sampling error for free field over reflecting plane measurements.

We also note from the data in Table 6, that decreasing the number of measurement positions generally does not alter the mean upward bias, but does significantly increase the measurement imprecision as suggested by the error model. This effect is emphasized in Figure 10 , where the average deviation and standard deviation data of Table 6 are plotted vs number of measurement positions for the A-weighted data. One notable exception to these trends is the result for NF-7 calculation procedure (nine measurement points, one on each of five sides, one at each of four corners, rectangular measurement surface). Here, the selection of measurement positions has been optimized to minimize bias on the average for the rectangular surface, but with the result that the increased number of measurement points does not significantly improve the precision of the measurement over that for five measurement positions (for this sample of data). From this we must conclude that the sound pressure level at the corner positions is correlated with the center points in an average sense.
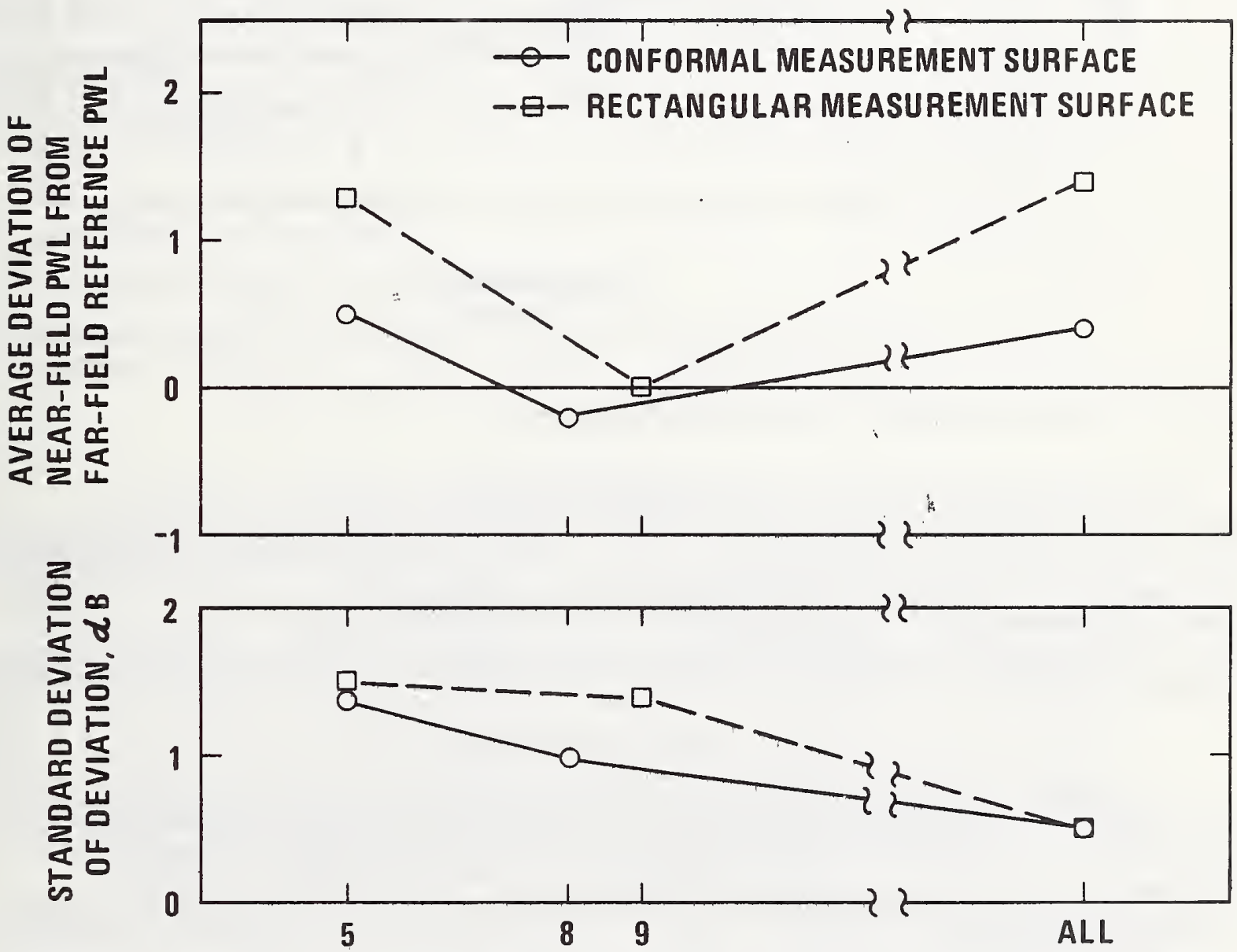

\section{NUMBER OF MEASUREMENT POSITIONS}

Figure 10 "Bias" and "precision of A-welghted sound power level vs number of measurement positions ( 17 compressors) 


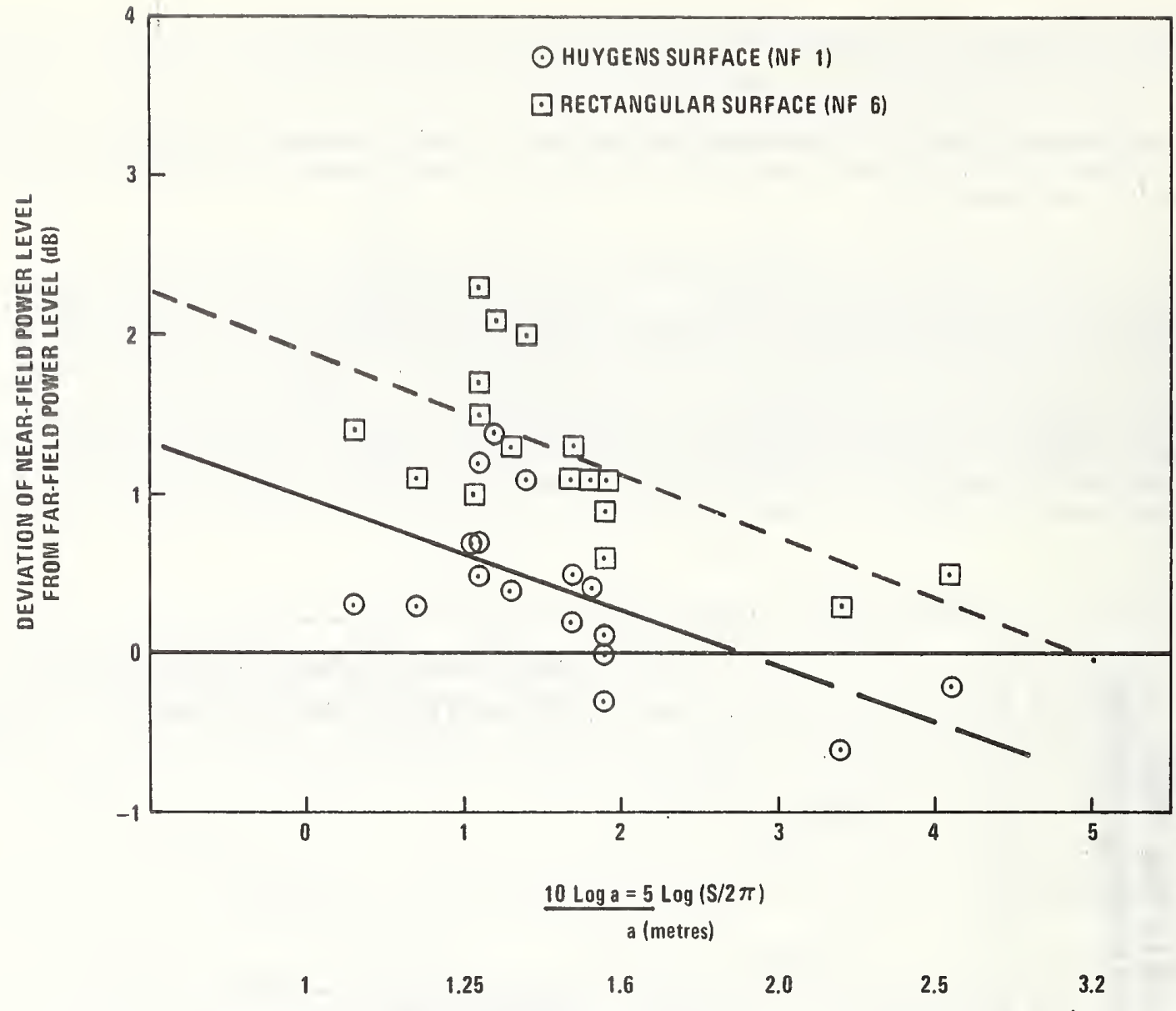

Figure 11 A-weighted sound power level deviation vs source size

Another possible type of bias in the measurement methodology is systematic variation as a function of machine size. Figure 11 shows a plot of deviation as a function of machine size for the NF-1 and NF-6 A-weighted sound power data. This plot indicates that both measurement methodologies have a significant tendency to overestimate sound power for smaller machines. This is. indicative of the dependence of the deviation from true sound power on measurement radius. However, it is probable that the microphone angular response problem is a factor in these data as well. Unfortunately, the data for larger machines is too sparse to provide accurate estimates of trends of deviation for very large machines, but eq. 5.6 suggests that both curves will have horizontal asymtotes at or above 0 aB deviation.

\subsection{Instrumentation Accuracy}

The analysis of measurement procedure error based on experimental data, discussed above, in effect eliminates instrumentation error except for microphone angular response, since we deal only with differences in levels measured with the same equipment. Errors due to bias from measurement system drift are minimized with frequent calibration, while bias from detection of signals is eliminated by using the same system in both measurements, etc. For measurement in the field environment however, these cancelations of error do not occur and must be considered in the estimate of total measurement error.

Table 7 provides a listing of pertinent sources of instrument error, their specified values for precision sound level meter and the range of expected error for presently avallable equipment.

The largest single source of error is tolerances on the A-weighting response, when that is used, since with proper microphone size, the error due to angular response can be minimized. Combining these errors leads to a total instrument imprecision (two standard deviations) on the order of 1.2 to $1.4 \mathrm{~dB}$ for tests made over a small temperature range from the temperature at calibration. (For calculations of total error we will use a value of 0.7 a for one standard deviation ( $90 \%$ confidence) for the instrument error component). 
Table 7. Instrument Imprecision (two Standard Deviations) Associated with Commercially Available Precision (Type I) Sound Level Meters

\begin{tabular}{|c|c|c|}
\hline Property & Standard* Iimits & Typical Limits \\
\hline Level Calibration & $\pm 0.2 \mathrm{~dB}$ & same \\
\hline $\begin{array}{l}\text { Frequency Response } \\
\text { (at each one-third } \\
\text { octave center } \\
\text { frequency }\end{array}$ & & \\
\hline $\begin{array}{l}\text { A-weighted } \\
\text { Linear }\end{array}$ & $\pm \frac{1}{ \pm} 0.5 \mathrm{~dB}$ & $\begin{array}{l} \pm 1 \mathrm{~dB}+ \\
\pm 0.5 \mathrm{~dB}\end{array}$ \\
\hline Attenuator Accuracy & $\pm 0.5 \mathrm{~dB}$ & $\pm 0.5 \mathrm{~dB}$ \\
\hline $\begin{array}{l}\text { Temperature Stability } \\
10^{\circ} \mathrm{C} \text { to } 60^{\circ} \mathrm{C} \\
-10^{\circ} \mathrm{C} \text { to } 60^{\circ} \mathrm{C}\end{array}$ & $\pm 1 \frac{\mathrm{dB}}{1} \mathrm{~dB}$ to $-5 \mathrm{~dB}$ & $\begin{array}{l}\text { same } \\
\text { same }\end{array}$ \\
\hline $\begin{array}{l}\text { Detector Iinearity } \\
\text { Crest Factor: } 10 \\
\text { Crest Factor: } \sim 1.7\end{array}$ & $\pm 5^{--}$ & $\begin{array}{l} \pm .5 \mathrm{~dB} \text { to } \pm 1 \mathrm{~dB} \\
\pm .5 \mathrm{~dB} \text { t }\end{array}$ \\
\hline $\begin{array}{l}\text { Angular Response** } \\
\left( \pm 90^{\circ}, 50 \mathrm{~Hz}-10 \mathrm{kHz}\right) \\
\text { One inch microphone } \\
\text { One-half inch microphone }\end{array}$ & \pm 2.1 to $-4 d B$ & $\begin{array}{l}+ \text { I to }-9 \mathrm{~dB} \\
+0.5 \text { to }-4.5 \mathrm{~dB}\end{array}$ \\
\hline
\end{tabular}

* IEC 179, ANSI S1. 4 - 1971 [12]

+ Assuming Spectra Similar to those measured

* For spectra similar to those measured and allowable standard tolerances vs. Frequency .

\subsection{Total Measurement Error}

The total imprecision in the measurement procedure is equal to the rms total of the error components when they are uncorrelated.

$$
e_{\text {total }}=\left(e_{\text {Meth }}^{2}+e^{2} \text { Instr }+e^{2} \text { Operator }\right)^{1 / 2}
$$

The methodology imprecision may be estimated from the standard deviation of the difference between the two procedures. (Ignoring the contribution of variance of the far field measurements, if it is not in fact negligible, contributes to make the estimate more conservative,) The standard deviation of the deviation from the far field procedure (Sigma Dev., Table 6) is a nearly unbiased estimate of the standard deviation $(\sigma)$ of the methodology, assuming that the errors are normally distributed. Examination of the cumulative distributions of the data indicate that this normality assumption is a reasonable approximation. However, because of the small number of tests involved, there is uncertainty associated with this estimate. To be conservative, we choose to estimate the methodology imprecision as the upper end of the $95 \%$ confidence interval for $\sigma$.

Neglecting operator error, and using a value of .7 dB for one standard deviation of instrument error, the range in achievable procedure error for the measurement of A-weighted sound power level is estimated to be in the range 1.1 to $2.4 \mathrm{~dB}$ depending on the number of measurement positions used, as shown in Table 8 .

The fact that the achieved precision of $1.5 \mathrm{~dB}$ for the NBS Far-field Methodology (see Table 6) (using a hand held sound level meter, and six measurement points) agrees reasonably well with the computed total precision of the survey methods, represents some confirmation of the precision analysis. 
Table 8. Estimated achievable measurement error ( $95 \%$ confidence) for measurement of A-weighted sound power level of portable air compressors in a field test environment using a measurement surface 1 metre from the source surface excluding operator error[13].

\begin{tabular}{|c|c|c|c|c|}
\hline Measurement Methodology & $\begin{array}{l}\text { Methodology } t \\
\text { Precision, } \mathrm{dB} \\
\text { (I standard } \\
\text { deviation) }\end{array}$ & $\begin{array}{l}\text { Instrument* } \\
\text { Precision, } \mathrm{dB} \\
\text { (1 standard } \\
\text { deviation) }\end{array}$ & $\begin{array}{l}\text { Total } \\
\text { Precision } \\
\text { (1 standard } \\
\text { deviation) }\end{array}$ & $\begin{array}{l}\text { Probable Inter- } \\
\text { val of Bias** } \\
\text { ( } 90 \% \text { Confidence) }\end{array}$ \\
\hline \multicolumn{5}{|l|}{ Conformal Surface } \\
\hline $\begin{array}{l}\text { Laboratory grade } \\
\text { ( } 1 \text { measurement per } \\
\text { square metre of } \\
\text { measurement surface) }\end{array}$ & .8 & .7 & 1.1 & -.3 to 1.1 \\
\hline $\begin{array}{l}\text { Engineering grade } \\
\text { (eight positions) }\end{array}$ & 1.5 & .7 & 1.7 & -1.6 to +1.2 \\
\hline $\begin{array}{l}\text { Survey grade } \\
\text { (five positions) }\end{array}$ & 2.3 & .7 & 2.4 & -1.5 to +2.5 \\
\hline Rectangular Surface & & & & \\
\hline $\begin{array}{l}\text { Laboratory grade } \\
\text { ( I measurement per } \\
\text { square metre of } \\
\text { measurement surface) }\end{array}$ & .8 & .7 & 1.1 & +.6 to 2.0 \\
\hline $\begin{array}{l}\text { Engineering grade } \\
\text { (nine positions) }\end{array}$ & 2.1 & .7 & 2.2 & -2.0 to +2.0 \\
\hline $\begin{array}{l}\text { Survey grade } \\
\text { (five positions) }\end{array}$ & 2.3 & .7 & 2.4 & -2.0 to +3.8 \\
\hline
\end{tabular}

+ Estimated from data of Table 6 using the expression (see text section 5)

$$
\sigma=1.52 \mathrm{~S}(\mathrm{Table} 6)
$$

where 1.52 is the upper limit of the $95 \%$ confidence interval for $\sigma / S$ based on $S$ with 16 degrees of freedom (14).

* Assumes Type I sound level meter (see text, Section 6).

* See reference 13, paragraph 3. 


\section{CONCLUSIONS}

The experimental data and analysis provided in this report support the following conclusions:

(1) An estimate of the "far field" sound power level output of a machine of a type similar to the compressors studied can be accurately inferred from sound pressure level measurements taken on a measurement surface close to the source if:

(a) the measurement surface conforms to the source shape;

(b) a sufficient number of measurement positions are used consistent with the precision requirements of the measurement;

(c) a microphone is utilized which has uniform response to sound, incident over any directions in front of the microphone over the frequency range of interest.

(2) Generally, the more measurement points that are used in a sound power level measurement the more precise the value of estimated sound power.

(3) Considering total invested measurement cost, imprecision of the results, and ease of microphone placement, the ISO Draft International Standard (DIS 3744) engineering method for sources using a conformal surface represents a most reasonable balance of these factors for making noise emission measurements for regulatory purposes.

(4) For the sample of compressors tested, the overall measurement precision (one standard deviation), in the field environment using the methodology of conclusion ( 3 ), is estimated to be $\pm 1.7 \mathrm{~dB}(95 \%$ confidence limits $3.4 \mathrm{~dB})$ ignoring instrument operator error. 
In any program of this Bize and scope, a large number of individuals contribute to ito success. The author is particularly indebted to the following 1ndividuals and organdzations for the1r contributions to this program.

Appreciation is expressed to the U. S. Army, Mobile Equipment Research and Development Command, Fort Belvolr, Virginia, for providing the use of a suitable test site and assistance in the recelpt and shipment of test specimens.

The author also expresses appreciation to Mr. D. E. Mathews for design and to the NBS Plant Division, for construction of the far-field array; and to the following members of the Mechanics Division, NBS: D. E. Mathews, B. R. Fuller, C. O. Shoemaker, and N. Rekos for their ass1stance during the data acquisition phase of the project; G. Hruska for calibration of the microphone cartridges; R. L. Fisher for programing and syatems analysis of the data acquisition system; D. S. Blomquist for instrumentation design and guidance; J. S. Forrer, J. M. Heinen, C. O. Shoemaker, and M. Tarica for instrumentation construction; C. T. Molloy (EPA/ONAC), D. R. Flynn, R. K. Cook and E. B. Magrab for suggestions to improve the theoretical analysis; D. M. Corley for assistance with data analysis and reduction; M. A. Cadoff and W. A. Leasure for editorial review of the manuscript; and J. Russell, $Y$. Morosko, and M. Hildebrand for typing and assembling the manuscript. 


\section{REFEREINCES}

[1] American National Standard for the Physical Measurement of Sound, S1.2-1962, (American National Standards Institute, New York, New York, 1971).

[2] International Organization for Standardization Draft Standard for Acoustics -- Determination of Sound Power Levels of Noise Sources. Part 4: Engineering Methods for Free-Field Conditions Over a Reflecting Plane, DIS-3744 (American National Standards Institute, New York, New York); International Organization for Standardization Draft Standard for Acoustics -Determination of Sound Power Levels of Noise Sources. Part 6: Survey Method for In Situ Measurements, DIS-3746 (American National Standards Institute New York, New York).

[3] For example: Symposium on sound power measurement of machines "in situ", J. Acous. Soc. Am. 54 (4) (1973) p. 960-984.

[4] Patterson, W. N., Ely, R., and Huggins, G., Portable air compressor noise measurement, Report 2795a (Bolt Beranek and Newman, Inc., Cambridge, Massachusetts, March 1974).

[5] Kearney, A. T., Inc., A study to determine the economic impact of noise emission standards in the construction equipment industry -- Draft portable air compressor report (U. S. Environmental Protection Agency, Washington, D. C., December 1973).

[6] American National Standard Test Code for the Measurement of Sound from Pneumatic Equipment, S5.1-1971 (American National Standards Institute, New York, New York, 1971).

[7] Baade, P. K., Standardization of machinery sound measurement, ASME Paper 69-WA/FE-30 (American Society for Mechanical Engineers, New York, New York, November 1969).

[8] Beranek, L. L., (ed.), Noise and Vibration Control, Chapter 6, Section 6.10 (McGraw-Hill, New York, New York, 1971, 158-163).

[9] Morse, P., and Ingaard, K. U., Theoretical Acoustics, Chapters 6 and 7 (McGraw-Hill, New York, New York, 1968).

[10] Skudrzyk, E., Foundations of Acoustics (Springer-Verlag, New York, New York, 1971).

[11] Felsen, L. B. and Marcuvitz, N., Radiation and Scattering of Waves, Chapter 1, (Prentice Hall, Englewood Cliffs, N. J., 1973).

[12] IEC 179 - International Electrotechnical Commission Recommendation Publication 179 (1965)

Precision Sound Level Meters, ANS S1.4-1971 - American National Standard S1.4 (1971) Specification for Sound Level Meters.

[13] Proschan, F., Confidence and tolerance intervals for the normal distribution, NBS Special Publication 300 (National Bureau of Standards, Washington, D.C.).

[14] Pearson, E. S., and Hartley, H. 0., ed. Biometricka, Tables for Statisticians, Vol. 1. (Cambridge University Press, 1958) Table 35, p. 184, Moments of S/ $\sigma$. 
NBS.114A (TEE, T.7A)

\begin{tabular}{|c|c|c|}
\hline $\begin{array}{l}\text { U.S. DEPT. OF COMM. } \\
\text { WBLIOGRAPHIC DATA } \\
\text { SHEET }\end{array}$ & $\begin{array}{l}\text { 1. 1'IIBI.I ATION OR RII'OR'T NO. } \\
\text { NBSIR 75-652 } \\
\text { EPA } 550.8=76=001\end{array}$ & 3. Recipicat's Accussion No. \\
\hline \multicolumn{2}{|c|}{$\begin{array}{l}\text { 4. TIII: ANI) S(IIITI.I: } \\
\text { PROCEDURES FOR ESTIMATING SOUND POWER FROM MEASUREMENTS OF } \\
\text { SOUND PRESSURE } \\
\text { An Experimental Investigation with Application to Noise From } \\
\text { Portable Air Compressors }\end{array}$} & $\begin{array}{l}\text { 5. Publication Date } \\
\text { Jan. } 1975 \\
\text { 6. Performing Organization Code }\end{array}$ \\
\hline \multicolumn{2}{|l|}{$\begin{array}{l}\text { 7. AUt'HOR(S) } \\
\text { Curtis I. Holmer }\end{array}$} & 8. Performing Organ. Report No. \\
\hline \multicolumn{2}{|c|}{$\begin{array}{l}\text { 9. PFRFORMING ORGANI7ATION NAME: AND ADDR LISS } \\
\text { NATIONAL BUREAU OF STANDARDS } \\
\text { DEPARTMENT OF COMMERCE } \\
\text { WASHINGTON, D.C. } 20234\end{array}$} & $\begin{array}{l}\begin{array}{l}\text { 10. Projuct/Task/Work Unit No. } \\
2130152\end{array} \\
\text { 11. Contract/Cirant No. }\end{array}$ \\
\hline \multicolumn{2}{|c|}{$\begin{array}{l}\text { 12. Spunsoring Organization Name and (iomplete Address (Street, City, State, ZIP) } \\
\text { Joint NBS and Environmental Protection Agency (EPA) } \\
\text { Office of Noise Abatement } \\
\text { Washington, D. C. } 20460\end{array}$} & $\begin{array}{l}\text { 13. Type of Report \& Period } \\
\text { Covercd } \\
\text { Final report } \\
\text { Feb. } 1974=\text { Dec } 1974 \\
\text { 14. Sponsuring Agency Code }\end{array}$ \\
\hline
\end{tabular}

15. SUPPI.T:MI:NTARY NOTISS

16. ABSTRAC"I (A 200-word or less factual summary of most significant information. If document includes a significant bibliography or literature survey, mention it here.)

This report describes investigations of the accuracy and precision of various measurement methodologies for determining the estimated sound power output of "large" machines in the free field over a reflecting plane. One purpose of this investigation is to place empirical error bounds on many of the free field measurement procedures currently proposed or in use; and in particular, compare the results of "near-field" and "far-field" measurements. The sources used for the investigation included 17 portable air compressors of various types (powered by internal combustion engines), a "reference" sound source, and a loudspeaker driven by a pure tone source. The data recorded include sound pressure level (A-weighted, linear, and 1/3-octave band) on an 84 point hemispherical array of seven metre radius, and "near-field" measurements, sampled every square metre, on a rectangular surface one metre from the machine surface. These data were reduced to provide information on the deviation of "near field" sound power determinations from "far-field" power level (using subsets of the data as appropriate to various methodologies). The measured data for seventeen sources suggests that the value of a sound power estimate based on "near-field" sound pressure level measurements may be an upper bound to the sound power level estimated from far field measurements, subject to the limitations of sampling error. Estimates of total achievable measurement error of A-weighted sound power level of near field determinations relative to far field determinations are made for several measurement methodologies, based on the experimental data.

17. KEY WORDS (six to twelve entries; alphabetical order; capitalize only the first letter of the first key word unless a proper name; separated by semicolons) Air compressors; error of sound power measurement; noise; noise measurement; sound power level; standard test procedures for sound power measurement.

18. AVAILABILITY X Unlimited

For Official Distribution. Do Not Release to NTIS

Order From Sup. of Doc., U.S. Government Printing Office Washington, D.C. 20402, SD Cat. No.C13

X Order From National Technical Information Service (NTIS) Springfield, Virginia 22151

\begin{tabular}{|l|c|}
\hline $\begin{array}{l}\text { 19. SECURITY CLASS } \\
\text { (THIS REPURT) }\end{array}$ & 21. NO. OF PAGES \\
UNCL ASSIFIED & 78 \\
\hline $\begin{array}{l}\text { 20. SECURITY CLASS } \\
\text { (THIS PAGE) } \\
\text { UNCLASSIFIED }\end{array}$ & 22. Price \\
$\$ 4.75$ \\
\hline
\end{tabular}

